\title{
Regulation of Respiratory Chain Supercomplex Formation and the Involvement of Rcf-proteins
}

\author{
DISSERTATION \\ for the award of the degree \\ "Doctor rerum naturalium" \\ at the Georg-August-Universität Göttingen \\ within the doctoral programme "Molecular Medicine" \\ of the Georg-August University School of Science (GAUSS) \\ submitted by \\ Bettina Homberg \\ born in Würzburg, Germany
}

Göttingen, 2021 

Thesis advisory committee

$\begin{array}{ll}\text { Dr. Markus Deckers } & \text { Institute for Cellular Biochemistry } \\ \text { (Supervisor and first referee) } & \text { University Medical Center, Göttingen } \\ & \text { Institute of Biochemistry and Signal Transduction } \\ & \text { University Medical Center, Hamburg-Eppendorf }\end{array}$

Prof. Dr. Dörthe Katschinski Institute of Cardiovascular Physiology

(Second referee)

Prof. Dr. Stefan Jakobs

\section{Further members of the examination board}

Prof. Dr. Ralph Kehlenbach

Prof. Dr. Henning Urlaub

Dr. Dieter Klopfenstein

Day of the oral examination: 19.05 .2021
Institute for Molecular Biology

University Medical Center, Göttingen

Bioanalytical Mass Spectrometry

Institute for Biophysical Chemistry

Max-Planck Institute, Göttingen

Third Institute for Physics,

Department of Biophysics

University of Göttingen 


\section{Affidavit}

I hereby declare, that my dissertation "Regulation of respiratory chain supercomplex formation and the involvement of Rcf-proteins" has been written independently and with no other sources and aids than quoted.

Bettina Homberg, Göttingen, March 2021

Parts of this thesis are communicated in the following publications:

Linden A., Deckers M., Parfentev I., Pflanz R., Homberg B., Neumann P., Ficner R., Rehling P., Urlaub H. (2020). A cross-linking mass spectrometry approach defines protein interactions in yeast mitochondria. Molecular \& Cellular Proteomics, 19(7), 1161-1178.

Homberg B., Römpler K., Wissel M., Callegari S., Deckers M. Rcf proteins in the assembly and organization of respiratory chain supercomplexes. (under review) 


\section{Table of Contents}

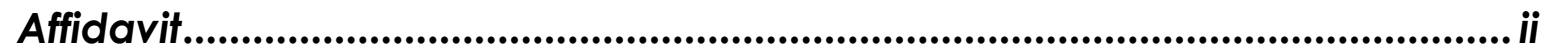

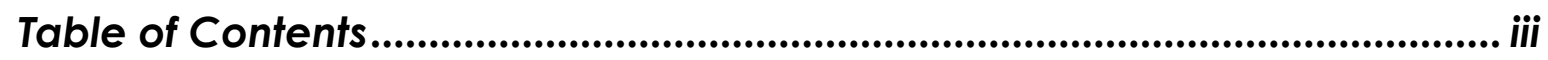

List of Figures ............................................................................................................ vii

List of Tables...................................................................................................... viii

List of Abbreviations................................................................................................... ix

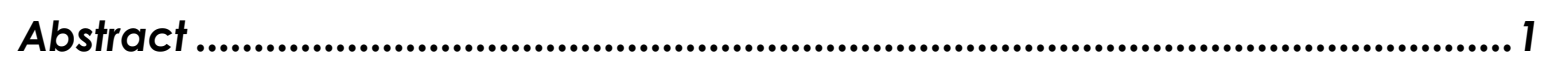

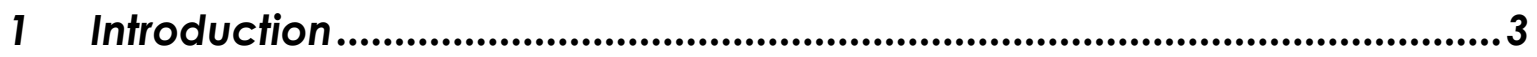

$1.1 \quad$ The eukaryotic cell .............................................................................. 3

1.2 Mitochondria - structure and function ..................................................... 3

1.3 Mitochondrial proteins - dual genetic origin ............................................... 5

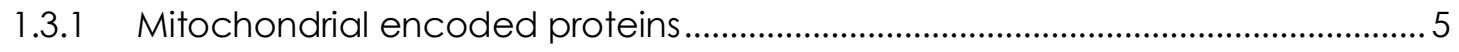

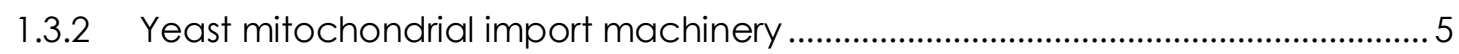

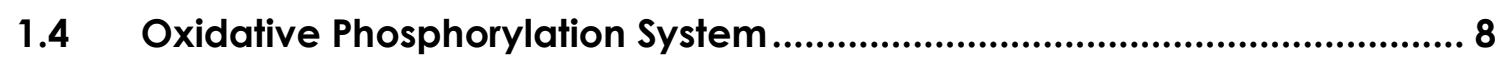

1.4.1 Structure and function of the respiratory chain ....................................................

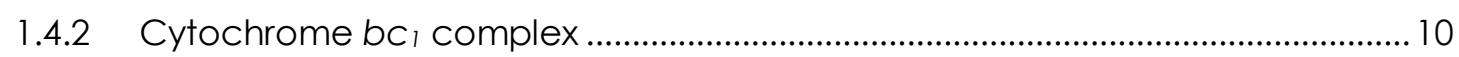

1.4.3 Cytochrome c Oxidase - function and biogenesis ............................................ 10

1.4.4 Cytochrome c oxidase - oxygen sensitive subunits............................................... 13

$1.5 \quad$ Respiratory supercomplexes ......................................................................14

1.5.1 Structure of respiratory supercomplexes .............................................................. 16

1.5.2 Function and biogenesis of respiratory supercomplexes.................................... 17

1.5.3 Respiratory supercomplexes - assembly and stabilizing factors .......................... 18

1.6 Aims of this study ......................................................................................21

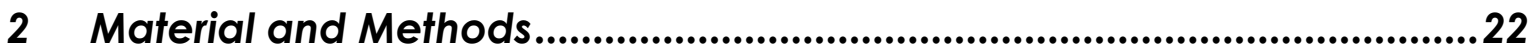

$2.1 \quad$ Material................................................................................................22

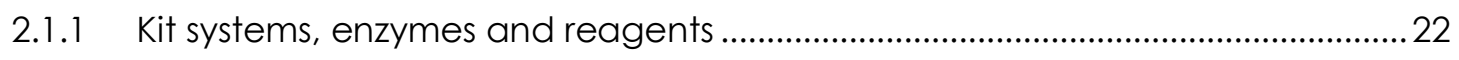

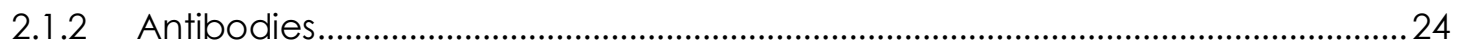

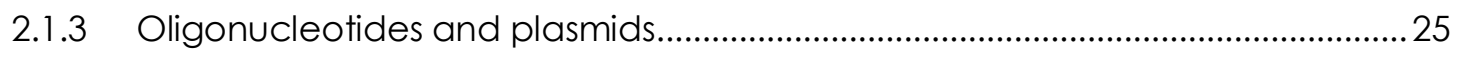

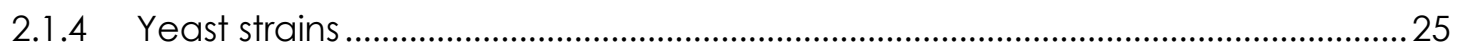

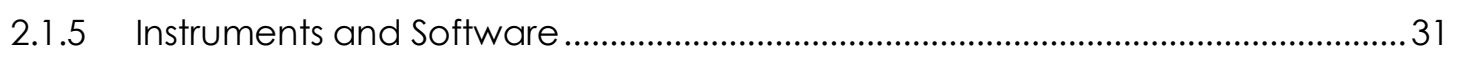




\subsection{Methods}

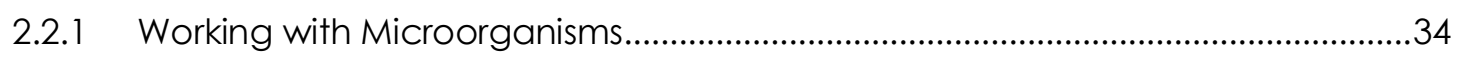

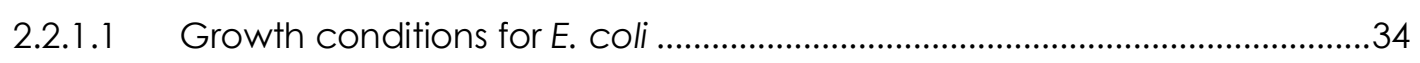

2.2.1.2 General handling and growth conditions for yeast .....................................34

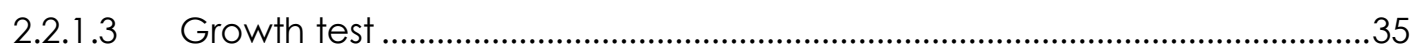

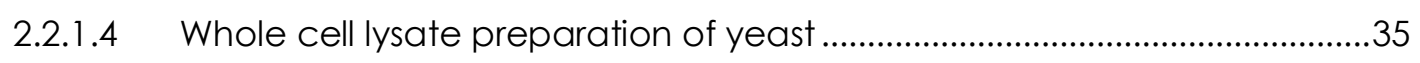

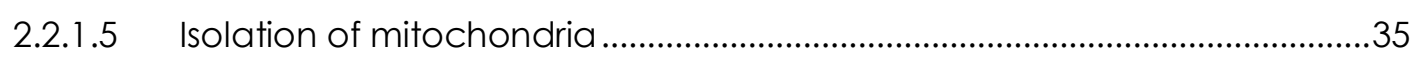

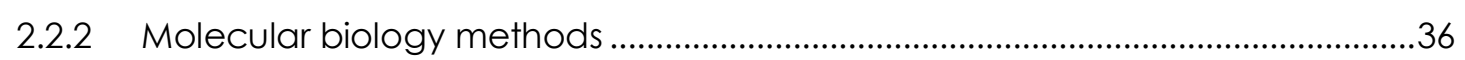

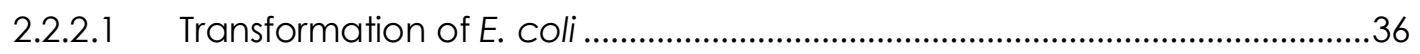

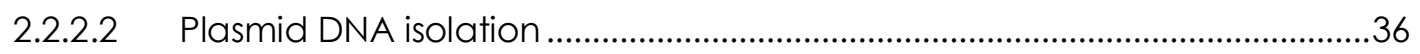

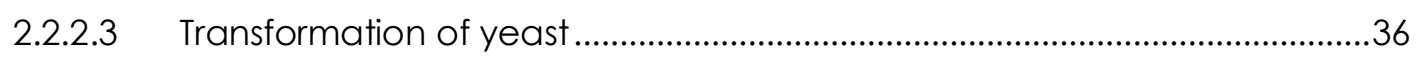

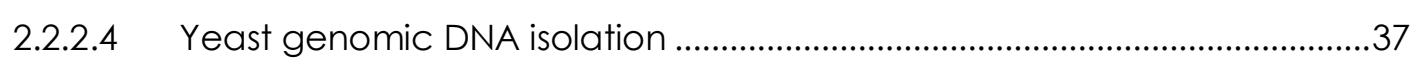

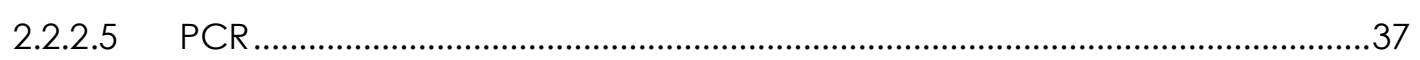

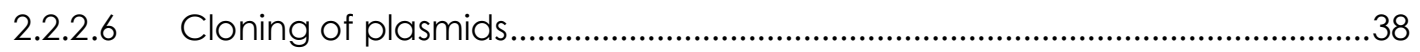

2.2.2.7 Chromosomal deletions/insertions in yeast.......................................................38

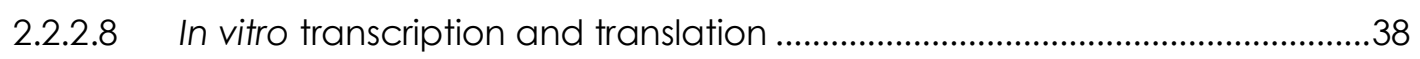

2.2.3 Protein biochemistry methods .............................................................................39

2.2.3.1 Bradford assay - determination of protein concentration ..........................39

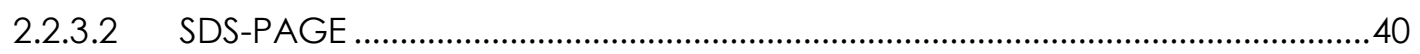

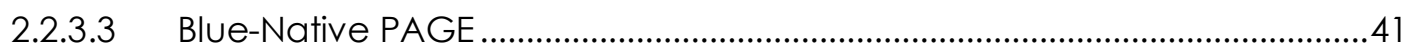

2.2.3.4 Western blotting and immunodetection ......................................................... 41

2.2.3.5 Coomassie staining of membranes and gels ................................................42

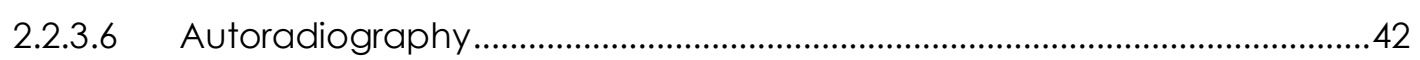

2.2.3.7 Steady state analysis of protein levels..........................................................42

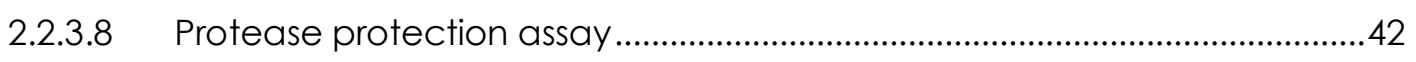

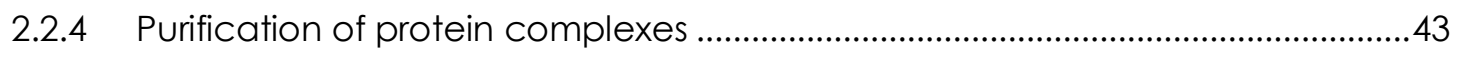

2.2.4.1 Crosslinking of Antibodies to PA-Sepharose beads ........................................43

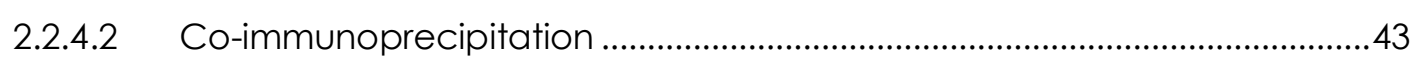

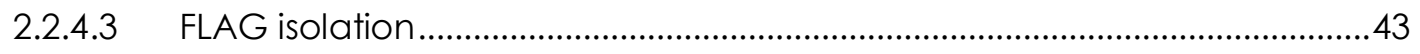

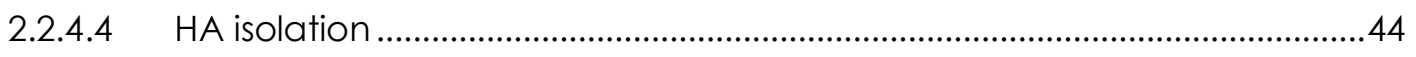

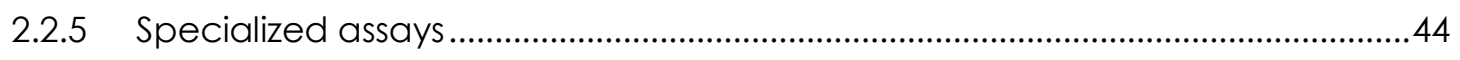

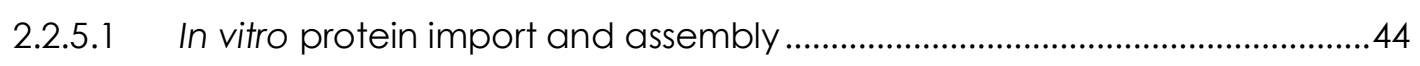

2.2.5.2 In vitro translation assay in isolated mitochondria ........................................44

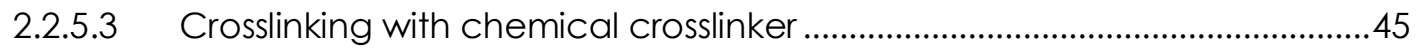

2.2.5.4 Determination of oxygen consumption rates.................................................45

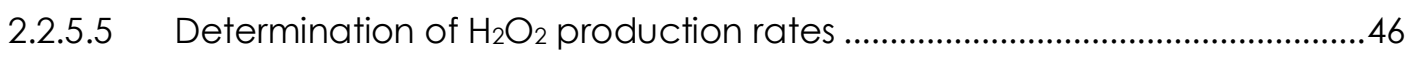




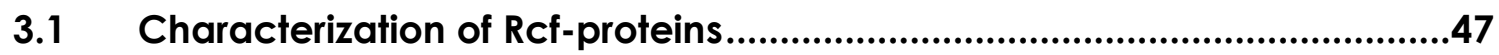

3.1.1 Rcf-proteins share similar orientation within mitochondria ..................................47

3.1.2 Rcf-proteins and their influence on respiration ........................................................ 49

3.1.3 Rcf-proteins interact with newly synthesized complex IV core subunits .............51

3.2 Crosslinks reveal Rcf2 and Rcf3 in close interaction with COX subunits ...53

3.2.1 Rcf2 crosslinks specifically to Cox12 and Cox13 ...................................................5

3.2.2 Rcf3 specifically crosslinks to Cox12 ........................................................................ 58

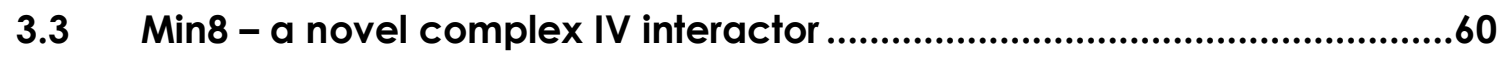

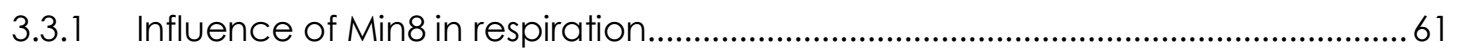

3.3.2 Min8 affects Cox12 assembly into cytochrome $c$ oxidase .................................64

3.4 Functionality assessment of the Rcf-domains........................................66

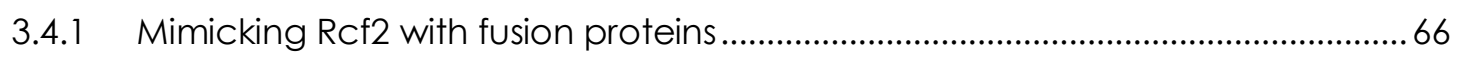

3.4.2 Rcf3-Rcfl, Rcf2N-Rcfl and Rcf3-Rcf2C are functional mitochondrial proteins.. 67

3.4.3 Rcf2N-Rcfl and Rcf3-Rcf1 facilitate supercomplex assembly ...............................70

3.4.4 Rcf3-Rcfl and Rcf2N-Rcf1 support Cox13 assembly.............................................

3.4.5 Fusion proteins act flexible upon respiration............................................................. 72

3.4.6 Rcf2 fragments Rcf2 ${ }^{\mathrm{N}}$ and Rcf2C alone are not functional.................................. 74

3.4.7 Oxygen consumption and ROS measurements reveal dynamic functions of

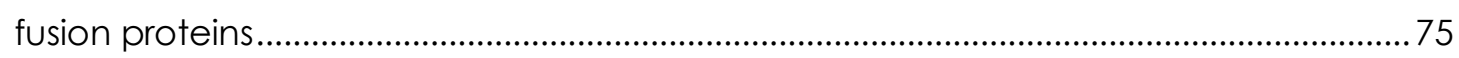

3.4.8 Fusion proteins influence Rcfl association with newly translated Cox3 ............78

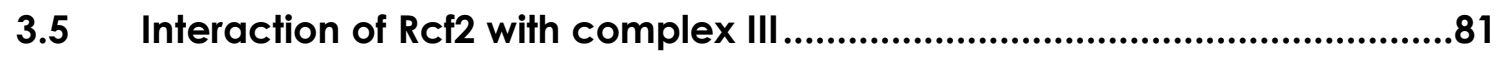

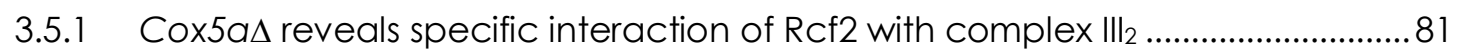

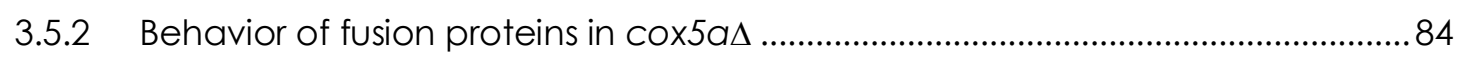

3.5.3 Tracing processed Rcf2 in the cox5as background.............................................. 85

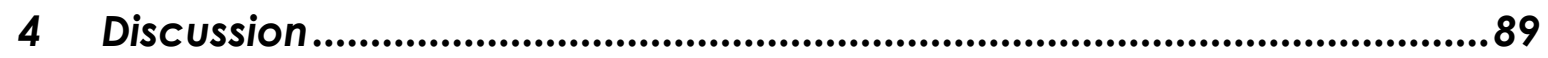

4.1 Topology and orientation of the Rcf-proteins ........................................89

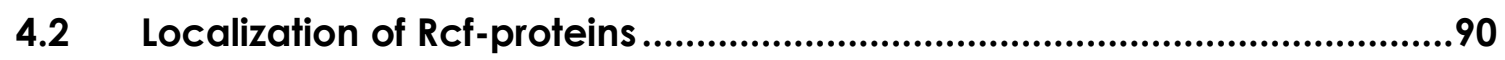

4.2.1 Rcf-proteins as substoichiometric interactors of COX...........................................90

4.2.2 The interaction network of Rcf2 and Rcf3......................................................... 91

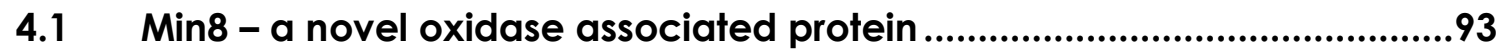

4.1.1 Interaction with COX - a new complex IV subunit? .............................................93

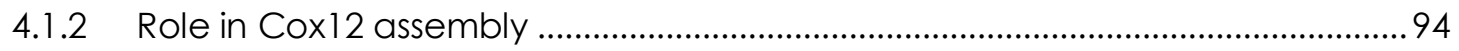


4.2 Fusion proteins as a tool to study Rcf-functions ......................................96

4.3 Rcf-proteins - genuine respiratory supercomplex factors? .....................97

4.3.1 Rcf1 - Dual role in supercomplex assembly ............................................................97

4.3.2 Rcf2 - regulation and assembly factor .................................................................... 101

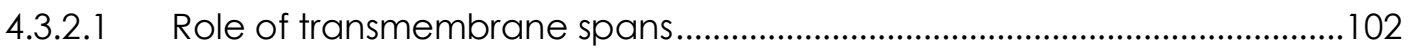

4.3.2.2 Role of internal processing event.................................................................. 103

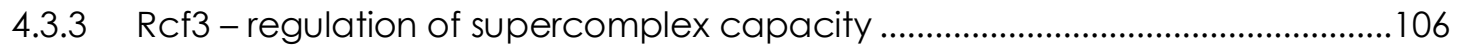

4.4 Role of Rcf-proteins under stressing conditions ................................... 107

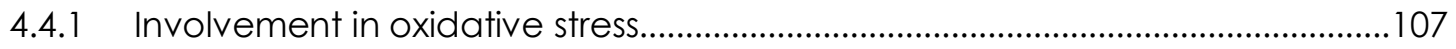

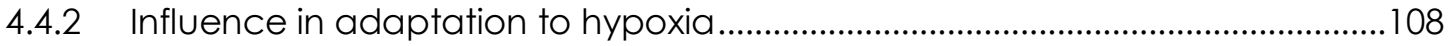

5 Summary and Conclusion ............................................................. 111

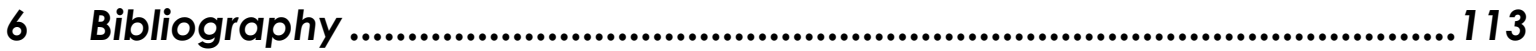

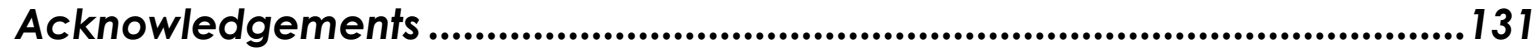

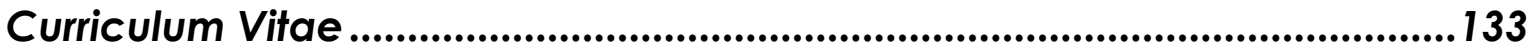




\section{List of Figures}

Figure 1-1: Overview mitochondrial compartments.

Figure 1-2: Main import pathways for nuclear encoded mitochondrial proteins - overview...... 6

Figure 1-3: Schematic overview of oxidative phosphorylation machinery in mammals and yeast.

Figure 1-4: Model - overview of yeast cytochrome $c$ oxidase modular assembly........................ 11

Figure 1-5: Model - structure of mammalian and yeast respiratory chain complexes................17

Figure 3-1: Rcf-proteins share homologous domains and similar orientation within mitochondria. 48

Figure 3-2: Rcf1 is a vital protein for respiration, Rcf2 and Rcf3 only in combination.

Figure 3-3: Supercomplex assembly is affected in $\operatorname{rrfl} \Delta$, not reflected by a major decrease in COX subunits. 50

Figure 3-4: Rcf-proteins co-isolate mitochondrial encoded proteins. 52

Figure 3-5: Rcf2 and Rcf3 are crosslinked to Cox12 and Cox13. 54

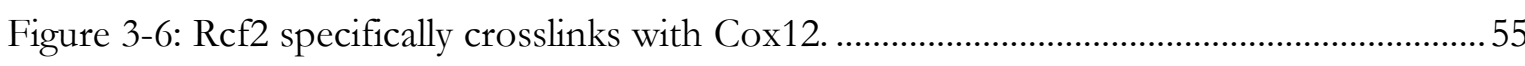

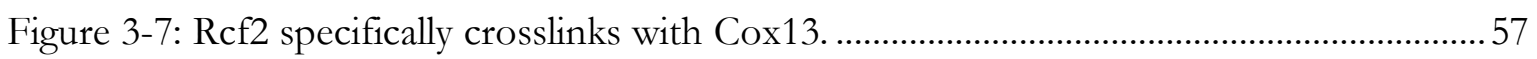

Figure 3-8: Structural analysis reveals crosslink of Rcf2 and Cox12 likely to happen at the

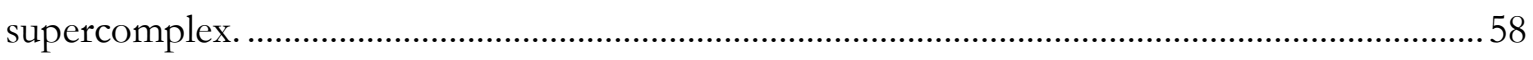

Figure 3-9: Rcf3 specifically crosslinks with Cox12.

Figure 3-10: The uncharacterized protein Min8 (YPR010C-A) is specifically crosslinked to Cox12. 60

Figure 3-11: Min8 is not essential for respiration. 62

Figure 3-12: MIN8 deletion does not affect respiration capacity. .63

Figure 3-13: Min8 influences late stage assembly of cytochrome $c$ oxidase. 65

Figure 3-14: Expressing fusion proteins in yeast.

Figure 3-15: Fusion proteins can substitute for Rcf-proteins. 68

Figure 3-16: Fusion proteins do not influence protein levels.

Figure 3-17: Fusion proteins colocalize at respiratory supercomplexes. 70

Figure 3-18: Expression of Rcf3-Rcf1 and Rcf2 ${ }^{\mathrm{N}}$-Rcf1 rescues $r f 1 \Delta$ phenotype in supercomplex arrangement.

Figure 3-19: Rcf3-Rcf1 and Rcf2 ${ }^{\mathrm{N}}$-Rcf1 compensate Cox13 assembly phenotype in $r f 1 \Delta$...... 72 Figure 3-20: Rcf3-Rcf1 and $\operatorname{Rcf2}{ }^{\mathrm{N}}-\mathrm{Rcf1}$ rescue $\operatorname{rcf} 1 \Delta$ and $\operatorname{rcf} 2 \Delta \operatorname{rcf3} \Delta$ respiration phenotype simultaneously

Figure 3-21: $\operatorname{Rcf} 2^{\mathrm{N}}$ and $\operatorname{Rcf}^{\mathrm{C}}$ fragments alone are not functional. 74 
Figure 3-22: Overexpressing parts of Rcf2 results in higher ROS production...... . .76

Figure 3-23 Fusion proteins modulate oxygen consumption and $\mathrm{H}_{2} \mathrm{O}_{2}$ production in a flexible way......

Figure 3-24: Rcf3-Rcf1 and Rcf2N-Rcf1 support post-translational interaction with Cox3.....79

Figure 3-25: Post-translational interaction of Rcf1 with Cox3 is impaired in $r f 2 \Delta r c f 3 \Delta$. . .80

Figure 3-26: Hypoxic isoform Cox5b cannot compensate for loss of Cox 5 a.

Figure 3-27: Rcf2 associates with complex $\mathrm{III}_{2}$ in $\operatorname{cox} 5 a \Delta$ while supercomplex assembly is impaired.

Figure 3-28: The minority of fusion proteins resides at $\mathrm{III}_{2} \mathrm{IV}$ and $\mathrm{Rcf}^{\mathrm{C}}$ co-migrates with $\mathrm{III}_{2}$.

Figure 3-29: Processed versions of Rcf2 associate with complex $\mathrm{III}_{2} \mathrm{IV}$ and $\mathrm{III}_{2}$ .86

Figure 4-1: Comparing resolved and modeled structures of yeast Rcf2, Min8 and human NDUFA4.

Figure 4-2: Model of Rcf-protein involvement with complex IV and supercomplex assembly. 100

Figure 4-3: Rcf2 processing - model of possible scenarios. 105

\section{List of Tables}

Table 2-1: Kit systems used in this study .22

Table 2-2: Special reagents and enzymes used in this study .........................................................22

Table 2-3: Commercially available antibodies used in this study.................................................24

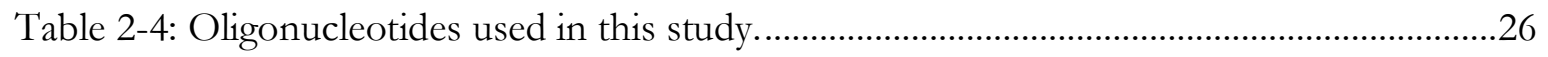

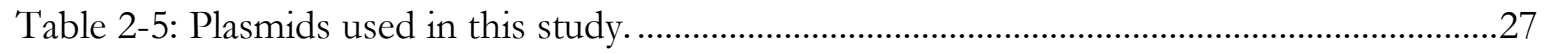

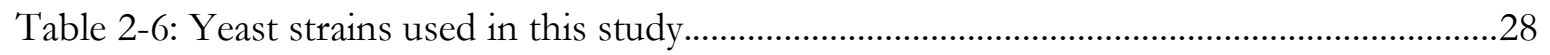

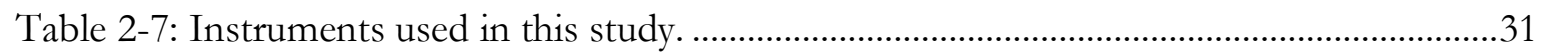

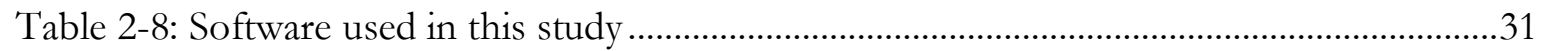

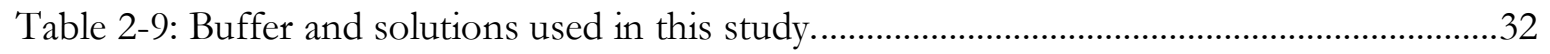

Table 2-10: Conditions for Flexi® Reticulocyte Lysate System. .................................................39 


\section{List of Abbreviations}

$\Delta \psi$

AA

ADP

APS

ATP

AVO

$\mathrm{BN}$

bp

$\mathrm{BS}^{3}$

BSA

COX/Cox

Cryo-EM

CSM

CWW

DMSO

DNA

DTT

ECL

EDTA

EM

g

HA

$\mathrm{HAc} / \mathrm{Ac}$

HEPES

HIG

HRP

$\operatorname{IgG}$

IMM

IMP

IMS

IP

LB
Mitochondrial membrane potential

Amino acid

Adenosine diphosphate

Ammonium persulfat

Adenosine triphosphate

Antimycin A, valinomycin, oligomycin

Blue native

basepair

Bis(sulfosuccinimidyl)suberate

Bovine serum albumine

Cytochrome $c$ oxidase

Cryo-electron microscopy

Complete supplement mixture

Cell wet weight

Dimethyl sulfoxide

Deoxyribonucleic acid

1-,4-Dithiotreitol

Enhanced chemiluminescence

Ethylenediaminetetraacetic acid

Buffer containing EDTA, MOPS

$g$-force

Human influenza hemagglutinin

Acetic acid/acetate

4-(2-hydroxyethyl)-1-piperazineethanesulfonic acid

Hypoxia induced gene

Horseradish peroxidase

Immunoglobulin $G$

Inner mitochondrial membrane

Inner membrane peptidase

Intermembrane space

Immunoprecipitation

Lysogeny broth 
Met

MIA

MICOS

MIM

MOPS

MPP

NEM

NHS

NMR

$\mathrm{OD}_{600}$

OMM

ORF

OXA

OXPHOS

PA

PAGE

PAM

PBS

PCR

PEG

PMSF

PVDF

RNA

ROS

rpm

SAM

Sat.

$\mathrm{SD} / \mathrm{G} / \mathrm{Gal}$

SDS

SEM

SEM

$\mathrm{SH}$

SORB

TBS/TBS-T
Methionine

Intermembrane space import and assembly machinery

Mitochondrial contact site and cristae organizing system

Mitochondrial import complex

3-(N)-morpholinopropanesulfonic acid

Mitochondrial processing peptidase

n-ethylmaleimide

N-Hydroxysuccininimide

Nuclear magnetic response

Optic density at $600 \mathrm{~nm}$

Outer mitochondrial membrane

Open reading frame

Oxidase assembly translocase

Oxidative phosphorylation

Protein A

Polyacrylamide gel electrophoresis

Presequence associated motor

Phosphate buffered saline

Polymerase chain reaction

Polyethylene glycol

Phenylmethylsulfonyl fluoride

Polyvinylidene fluoride

Ribonucleic acid

Reactive oxygen species

Rounds per minute

Sorting and assembly machinery

saturated

Synthetic dextrose/glycerol/galactose

Sodium dodecyl sulfate

Buffer containing sucrose, EDTA, MOPS

Standard error of the mean

Buffer containing sorbitol, HEPES

Buffer containing Sorbitol

TRIS buffered saline/TRIS buffered saline, Tween-20 
TCA

TEMED

TIM22

TIM23

TMPD

TOM/Tom

TRIS

UV

$\mathrm{V}$

Wt

YNB
Trichloroacetic acid

Tetramethylenediamine

Carrier translocase of the inner membrane

Presequence translocase of the inner membrane

$\mathrm{N}, \mathrm{N}, \mathrm{N}^{\prime}, \mathrm{N}^{\prime}$-tetramethyl-p-phenylenediamine

Translocase of the outer membrane

2-amino-2-(hydroxymethyl)propane-1,3-diol

ultraviolet

Volume [1]

Wild-type

Yeast nitrogen base

YPD/G/Gal/Lac Yeast extract peptone dextrose/glycerol/galactose/lactate 



\section{Abstract}

The mitochondrial oxidative phosphorylation machinery is comprised by five individual complexes and arranged in supramolecular structures, so-called supercomplexes, as it is shown for complex I, III, IV and V. In yeast $S$. cerevisiae complex I is substituted by three singular $\mathrm{NADH}$ dehydrogenases and therefore lacks multimeric complex I. Thus, it displays a suitable model for investigating the dynamics within supercomplex formation of complex III and IV. Although some players could be identified so far, it is mostly unknown which factors contribute to forming these complexes $\left(\mathrm{II}_{2} \mathrm{IV}_{(1-2)}\right)$. Besides, the mechanism behind re-/arrangement of the supercomplexes to adapt to different conditions such as changing oxygen levels remains obscure.

The yeast Rcf-proteins (respiratory supercomplex factors) Rcf1, Rcf2 and Rcf3 were identified as proteins with partly corresponding homologous domains and can interact independently with complex III and complex IV. This is a unique feature promoting an important role of the protein family for recruiting and/or association of the two complexes. Furthermore, they could serve as regulator of complex IV and prevent from undesired ROS production but the exact localization and functional domains remained unknown.

A crosslinking approach on isolated yeast mitochondria could identify the interacting site of Rcf2 and Rcf3 at the periphery of complex IV contrary to first suggestions for the association at the interface of complex III and IV. During this investigation we discovered a putative novel subunit of complex IV - Min8 - and proved it as being involved in complex IV biogenesis. For determining the functionality of the different domains of Rcf-proteins, artificial fusion proteins out of Rcf1, Rcf2 and Rcf3 were constructed and expressed in various mutational strains. The effects of those proteins were assessed by various experiments addressing the respiration, complex biogenesis and ROS production. While Rcf1 operates on both, complex IV and supercomplex assembly suggesting an even higher dynamic than originally anticipated, Rcf2 and Rcf3 are acting predominantly at the site of supercomplexes. At the same time, we discovered that the functionality of transmembrane regions of Rcf2 depend on the overall topology of the protein. By further studying the involvement of the respective protein domains in supercomplex assembly, we found an interesting interaction of Rcf2 and its processed versions at the site of complex $\mathrm{III}_{2}$ under alleged hypoxic conditions in a COX5 A mutant. This suggests a specific involvement within the adapting respiratory chain to altering oxygen levels. 



\section{Introduction}

\subsection{The eukaryotic cell}

There are three main domains which comprise all living organisms: Bacteria and archaea, as single-cell micro-organisms also named as prokaryotes, and eukaryotes. The unique feature of eukaryotes and in parallel the main difference to prokaryotes, is that they harbor a nucleus and a variety of other membrane coated organelles, compartmentalizing the particular enzymatic functions within the cell (Nelson and Cox, 2009; Gabaldón and Pittis, 2015). This leads to the evolutionary advantage that cellular, maybe conflicting, processes are separated and protected by lipid bilayers. Specializing the respective organellar functions allows highly efficient processes which can be differentiated by investigating the organelles themselves (Nelson and Cox, 2009). This in turn, can give valuable information about molecular and biochemical mechanisms in order to gain insight into processes in different tissues which are connected e.g. to diseases, their treatment or response to xenobiotics. Consequently, it was a revolutionary development when subcellular fractionation was accomplished by differential centrifugation steps - first, to isolate mitochondria enriched fractions and later refined for further discrimination (Claude, 1946; de Duve et al., 1955).

\subsection{Mitochondria - structure and function}

Mitochondria represent already an exceptional organelle by its unusual structure. They possess a double membrane which allows developing a proton gradient across the separate compartments, in the end essential for ATP synthesis. Studying isolated mitochondria became especially relevant since several conditions and diseases such as ageing, diabetes, neurodegenerative and cardiovascular diseases were associated with dysfunctional mitochondria (Schapira, 2006; Falabella et al., 2021). An important process also in this regard is the dynamic network of mitochondria, undergoing constant fission and fusion (Suárez-Rivero et al., 2016). The double-membrane of mitochondria and harboring an additional mitochondrial genome, led to the hypothesis that mitochondria originated from endosymbiosis of $\alpha$-proteobacteria. Furthermore, it was postulated that the presence of mitochondria and by this increased availability of energy, enabled the development of large cells and higher complexity (Gabaldón and Huynen, 2004; Lane and Martin, 2010). 


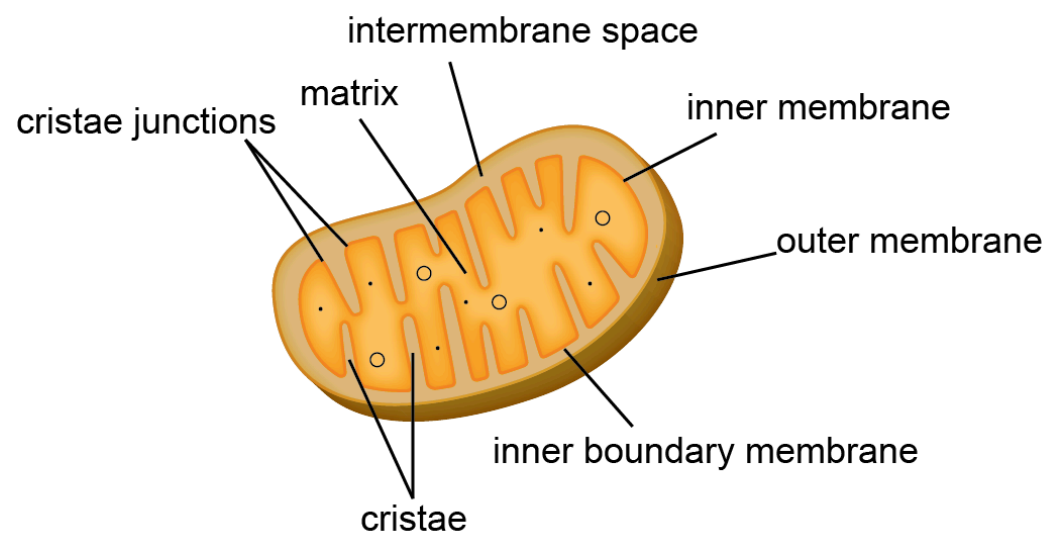

Figure 1-1: Overview mitochondrial compartments.

Mitochondria comprise four compartments: the outer membrane, the intermembrane space, the inner membrane and the matrix. The inner membrane possesses a large surface pronounced by invaginations called cristae, stabilized by cristae junctions, while the inner boundary membrane runs parallel to the outer membrane. The matrix houses the mitochondrial genome with the associated translation machinery.

Four main compartments can be identified within mitochondria: the outer mitochondrial membrane (OMM), the intermembrane space (IMS), the inner mitochondrial membrane (IMM) and the mitochondrial matrix (Figure 1-1). While the OMM is permeable for small molecules and ions through porin channels, the IMM is impermeable for most small molecules, including protons (Benz, 1994). Consequently, specific transporters and mechanisms are required for translocating molecules into the matrix but also inversely - from the matrix into the IMS and cytosol. The IMS as hydrophilic interspace between OMM and IMM harbors soluble proteins including chaperones involved in protein import (Wiedemann and Pfanner, 2017).

Embedded into the IMM lies the oxidative phosphorylation machinery (OXPHOS), comprising the respiratory chain complexes in addition to the ATP Synthase (ATPase). Characteristic for the protein-rich IMM is the large surface, accomplished by invaginations, subclassifying the membrane into the inner boundary membrane, cristae and cristae junctions which connect these domains. Responsible for stabilizing cristae junctions is the so-called MICOS (mitochondrial contact site and cristae organizing system) complex (Colina-Tenorio et al., 2020). It was also shown that this compartmentalization coexists with a distinct arrangement of the OXPHOS machinery in cristae while the translocase complexes reside at the inner boundary membrane aligned in parallel to the OMM (Vogel et al., 2006).

The mitochondrial matrix harbors the mitochondrial genome and the intrinsic expression machinery. Additionally, all fueling metabolism pathways of the eukaryotic cell except for glycolysis take place within the matrix - citrate cycle, fatty acid $\beta$-oxidation, amino acid oxidation, allowing an efficient delivery of substrates for the respiratory chain (Nelson and Cox, 2009). 
ATP synthesis is the most prominent function of mitochondria; it is the major source of energy for the cell. Nevertheless, the organelles are also involved in Fe-S protein maturation (Benz, 1994), steroid synthesis (Miller, 2013), $\mathrm{Ca}^{2+}$ homeostasis (Romero-Garcia and Prado-Garcia, 2019) and apoptosis induction (Wang and Youle, 2009) of the cell. Although we focus on the feature of the respiratory chain in this study, mitochondrial functions are quite divers and contribute to cellular maintenance by various essential mechanisms.

\subsection{Mitochondrial proteins - dual genetic origin}

Emerging from an endosymbiotic event, mitochondria transferred a large proportion of their genome into the nucleus. In parallel, an import system had to be established, ensuring the required supply of the mitochondrial proteins, now translated in the cytosol. However, mitochondria partly kept their own genome still encoding for 37 proteins in human and 35 proteins in yeast Saccharomyces cerevisiae (S. cerevisiae) (Malina, Larsson and Nielsen, 2018).

\subsubsection{Mitochondrial encoded proteins}

From S. cerevisiae to human, the mitochondrial genome is conserved with only few exceptions and encodes for proteins of the electron transport chain and oxidative phosphorylation, 22 tRNAs and 2 rRNAs, required for mitochondrial translation. S. cerevisiae lack multimeric NADH-Dehydrogenase (complex I), thus, genes encoding for subunits of complex I (in human ND1-6; 4L) are not present (Malina, Larsson and Nielsen, 2018). Expressed within yeast mitochondria is the ribosomal protein of the small subunit Var1 and ATPase subunit Atp9 additionally to the conserved cytochrome $c$ oxidase (complex IV) subunits Cox1, Cox2, Cox3; cytochrome $b c_{1}$ complex (complex III) subunit Cob and ATPase subunits Atp6, Atp8 (Malina, Larsson and Nielsen, 2018). It is assumed that these core proteins remained under mitochondrial regulatory control in order to respond quickly to environmental changes (Allen, 2015).

\subsubsection{Yeast mitochondrial import machinery}

The vast majority of mitochondrial proteins are translated on cytosolic ribosomes and need to be translocated through mitochondrial membranes. For this, specific import routes were developed which fulfill the responsibility transporting the diverse spectrum of mitochondrial proteins to their destination. These contain targeting signals stimulating the appropriate pathway (Wiedemann and Pfanner, 2017). 


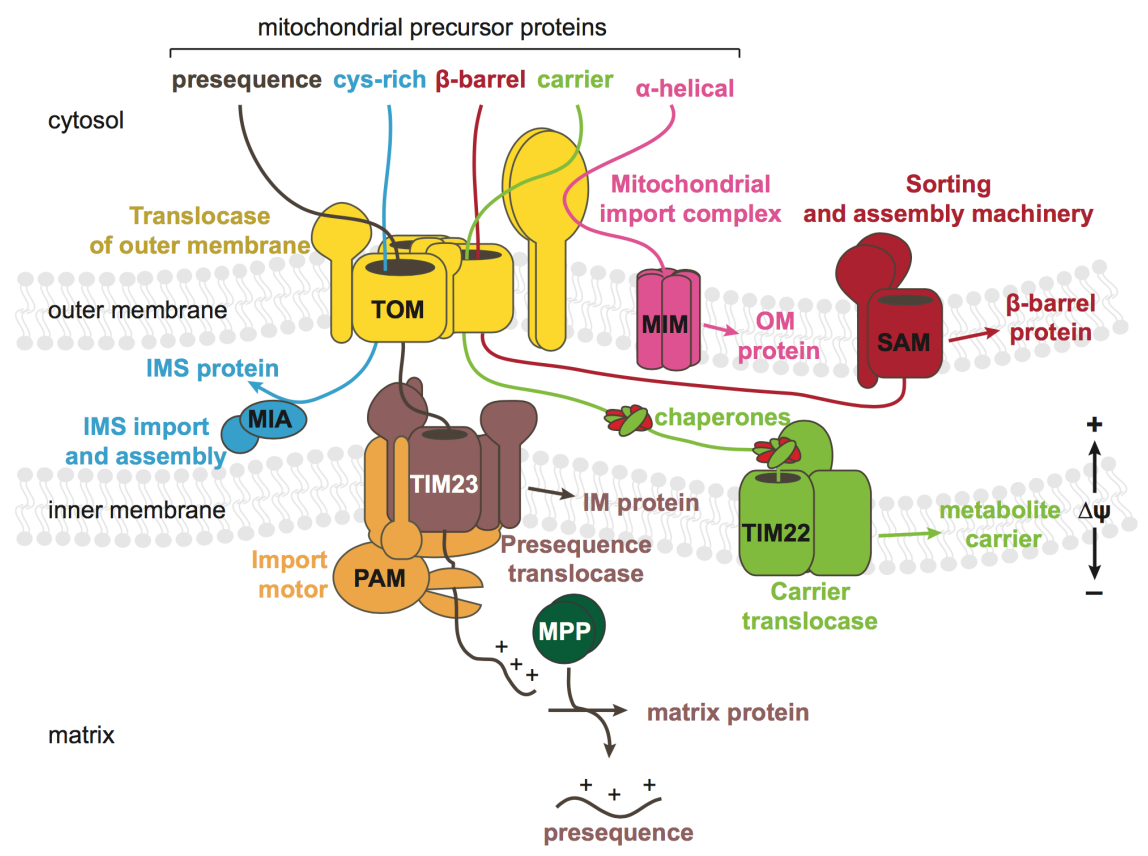

Figure 1-2: Main import pathways for nuclear encoded mitochondrial proteins - overview.

Precursor proteins carrying a presequence are translocated via TOM and TIM23 complexes, the presequence is cleaved by the peptidase MPP. Matrix protein import is driven by the Import motor PAM. Cysteine rich proteins are imported into the IMS via TOM and MIA. Carrier proteins are transported through the IMS by chaperones upon passing through TOM and handed to TIM22 complex for membrane insertion. Outer membrane proteins are inserted via MIM or TOM and SAM depending on their structure.

Translocase of outer mitochondrial membrane - TOM. The translocase of outer membrane (TOM) represents the predominant entry gate for the precursor proteins (Figure 1-2). Tom40 forms a channel through the OMM whereas Tom20, Tom 22 and Tom 70 represent specific receptors for presequence and non-cleavable precursors, respectively (Abe et al., 2000; Yamano et al., 2008; Wiedemann and Pfanner, 2017). The TOM complex forms certainly not a passive channel which allows passage through the OMM. In fact, it transports the proteins actively by hydrophobic and hydrophilic interplay (Hill et al., 1998; Shiota et al., 2015). According to the targeting signal the precursors are guided to the different compartments.

Translocase of inner mitochondrial membrane - TIM23. Most mitochondrial proteins have a cleavable $\mathrm{N}$-terminal presequence of various lengths, addressing the classical import pathway: the presequence pathway (Figure 1-2). Presequences contain an amphipathic $\alpha$-helix, recognized by TOM receptors (Abe et al., 2000; Yamano et al., 2008). After passing TOM, the protein is handed over to the presequence translocase of the inner mitochondrial membrane (TIM23). Two subsequent pathways are covered by TIM23 which are membrane potential dependent: protein transport into the matrix or embedding into the IMM from the IMS side. Main constituents are the pore building protein Tim23, together with the close interactor Tim17 
and the regulators Tim50 and Tim21, which is only involved in sorting TIM23 (Lytovchenko et al., 2013; Wiedemann and Pfanner, 2017). The proton motive force acts here by electrostatic effects on the positively charged presequence and by directly activating TIM23 via voltage dependent change of conformation (Martin, Mahlke and Pfanner, 1991; Malhotra et al., 2013). Subsequently, the presequence is removed by the mitochondrial processing peptidase (MPP) (Hawlitschek et al., 1988). Matrix proteins are translocated completely through the IMM with the help of the presequence translocase associated motor (PAM) in an ATP dependent manner. In contrast, IMM proteins using TIM23 are laterally released (sorted) with the help of a stop-transfer sequence and the membrane potential (van der Laan et al., 2007; Schulz, Schendzielorz and Rehling, 2015; Wiedemann and Pfanner, 2017). The hydrophobic sorting signal, in turn, can be cleaved by the inner membrane peptidase IMP and the protein is either released into the IMS or remains integrated within the IMM via additional transmembrane spans (Gakh, Cavadini and Isaya, 2002; Wiedemann and Pfanner, 2017). Interestingly, it was seen that sorting TIM23 interacts via Tim21 with complexes of electron transport chain, apparently promoted by the increased proton motive force in vicinity to the respiratory chain (reviewed in Schulz, Schendzielorz and Rehling, 2015).

Oxidase assembly translocase - OXA. IMM proteins which need to be inserted from the matrix side make use of the oxidase assembly (OXA) translocase. This is the case for mitochondrial encoded proteins but also the combined interplay together with the TIM23 presequence pathway was observed. The main component Oxa1 acts together with Mba1 on mitochondrial ribosomes facilitating co-translational protein insertion into the IMM (Ott and Herrmann, 2010). A cooperative import route with TIM23 is called the conservative sorting pathway. Here, IMM proteins are partly imported into the mitochondrial matrix by passing TIM23 with the help of PAM and subsequently exported by Oxa1 (Bohnert et al., 2010; Wiedemann and Pfanner, 2017).

Carrier translocase of the inner mitochondrial membrane - TIM22. Mitochondrial metabolite carriers are highly hydrophobic IMM proteins. They do not contain a cleavable presequence and are directed via several internal targeting signals (Brix et al., 1999; Endres, Neupert and Brunner, 1999; Wiedemann and Pfanner, 2017). Chaperones increase the solubility and the carriers can be transported through the cytosol and IMS (Figure 1-2). Initially, it was proposed that all carrier have six transmembrane spans but the spectrum was recently extended to carriers with less transmembrane spans (Gomkale et al., 2020). After entry through TOM, the 
protein is translocated through the IMS with the help of small TIM chaperones. Upon binding to the carrier translocase of the inner mitochondrial membrane (TIM22), the chaperones dissociate and the carrier is inserted into the IMM in a membrane potential dependent manner (Rehling, Brandner and Pfanner, 2004; Horten, Colina-Tenorio and Rampelt, 2020).

Intermembrane space import and assembly machinery - MIA. Various IMS proteins form a disulfide bond via characteristic cysteine motifs $\left(\mathrm{CX}_{3} \mathrm{C}, \mathrm{CX}_{9} \mathrm{C}\right)$ and are translocated along the mitochondrial intermembrane space import and assembly (MIA) pathway (Figure 1-2) (Chacinska et al., 2004). The precursors enter the TOM complex from the cytosol in a reduced state and Mia40 acts as a receptor from the IMS side. By utilizing its own redox-active cysteine pair, Mia40 establishes a mixed disulfide bond with the precursor which is transferred to the protein for correct folding. The factors Erv1 and Hot13 re-oxidize Mia40 for new substrate recognition (Mesecke et al., 2005; Fischer and Riemer, 2013). This pathway does not depend on the proton motive force of the inner membrane but solely on Mia40 and its the redox activity (Wiedemann and Pfanner, 2017).

Import of outer mitochondrial membrane proteins. Most outer mitochondrial membrane proteins are $\beta$-barrel proteins. Upon entry through TOM and transport through the IMS with small TIM chaperones, they are folded and inserted into the membrane via the sorting and assembly machinery SAM (Figure 1-2). $\alpha$-helical OMM protein import is accomplished via the mitochondrial import complex MIM, partly in cooperation with TOM. However, some tail-anchored OMM proteins seem to be embedded exclusively due to their hydrophobic feature (reviewed in Walther and Rapaport, 2009; Wiedemann and Pfanner, 2017).

\subsection{Oxidative Phosphorylation System}

The proton motive force of the inner mitochondrial membrane, as mentioned above, is the driving force of most protein import routes into mitochondria. It is built by a series of electron transferring redox reactions through the electron transport chain or respiratory chain, embedded in the IMM. However, key motivation is to drive the phosphorylating activity of ATP synthase to generate ATP - the universal energy resource of the cell (Rich and Maréchal, 2010; Neupane et al., 2019). 


\subsubsection{Structure and function of the respiratory chain}

Catabolic pathways such as glycolysis, citrate cycle and fatty acid $\beta$-oxidation deliver electrons by channeling them to the universal electron carrier $\mathrm{NAD}^{+}$. In principle, electron transport through the respiratory chain is achieved by various redox reactions via enzymes containing flavin nucleotides, Fe-S centers, hemes and copper centers (Rich and Maréchal, 2010). The mammalian respiratory chain comprises four complexes: NADH dehydrogenase (complex I), succinate dehydrogenase (complex II), cytochrome $b c_{1}$ complex or ubiquinol- cytochrome $c$ oxidoreductase (complex III) and cytochrome c oxidase (complex IV) (Figure 1-3A). The complexes were found to associate with each other in so-called supercomplexes. Various formations in mammalian and yeast mitochondria involving the complexes $\mathrm{I}-\mathrm{III}_{2}-\mathrm{IV}_{(1-4)}$ and $\mathrm{III}_{2}-\mathrm{IV}_{(1-2)}$, respectively, were obtained using mild detergents and native separation methods (see also section 1.5) (Schägger and Pfeiffer, 2000).
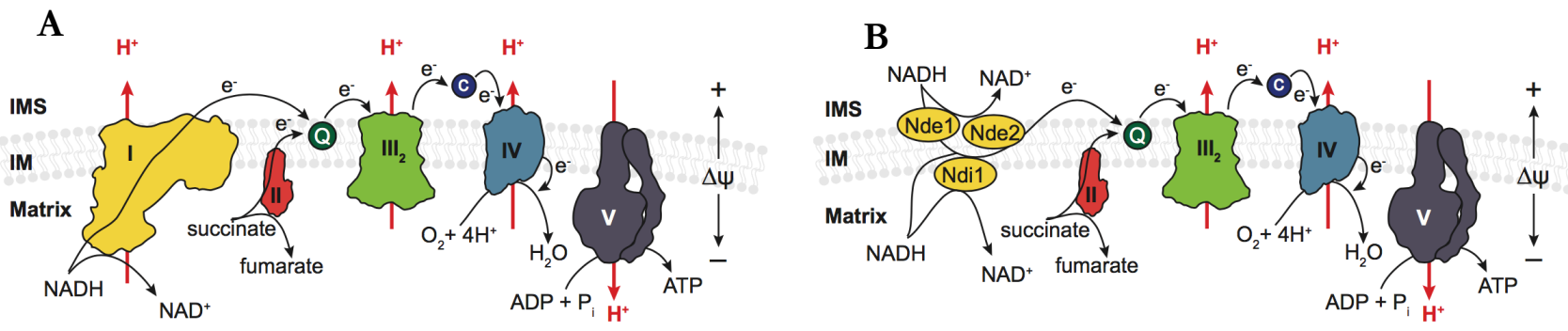

Figure 1-3: Schematic overview of oxidative phosphorylation machinery in mammals and yeast.

The mammalian oxidative phosphorylation system comprises the respiratory chain with complexes I-IV and complex V. Electrons enter via complex I and complex II, transferring them by a series of redox reactions. Co-enzyme Q and cytochrome $c$ act as electron shuttles transporting the electrons from complex I and II to complex III and from complex III to complex IV, respectively. The terminal reaction is the reduction of oxygen to water, accomplished by complex IV. During electron translocation, protons are pumped across the IM, generating a proton gradient. The energy from the gradient and back-flow of protons through complex $V$ drives ATP production. B) The yeast oxidative phosphorylation system functions similarly, except for the single NADH dehydrogenases (Nde1, Nde2, Ndi1) that substitute multimeric complex I.

Yeast S. cerevisiae lack multimeric complex I. Instead, NADH is oxidized by single NADH dehydrogenases Nde1, Nde2 and Ndi1 without proton translocation through the IMM (Figure 1-3B) (Grandier-Vazeille et al., 2001). Complex II represents the other direct electron entry of the respiratory chain. Coenzyme Q and cytochrome $c$ serve as free electron shuttles, transferring the electrons to complex III and from complex III to complex IV, respectively. While coenzyme $\mathrm{Q}$ as a hydrophobic co-factor diffuses through the membrane, cytochrome $c$ is soluble and binds to complex III and complex IV via IMS facing proteins. The cytochrome $c$ oxidase is the terminal enzyme using the transported electrons for reduction of molecular oxygen. Parallel to the presented redox reactions, protons are translocated through the IMM by 
complex I (in the mammalian system), complex III and complex IV. In the end, ATP production is driven by the back-flow of protons into the matrix through the $\mathrm{F}_{0} \mathrm{~F}_{1}$-ATP synthase (complex V) (Figure 1-3) (reviewed in Rich and Maréchal, 2010; Nolfi-Donegan, Braganza and Shiva, 2020).

\subsubsection{Cytochrome bc 1 complex}

The mitochondrially encoded cytochrome $b(\mathrm{Cob})$ represents the conserved core of complex III and forms together with the nuclear encoded Rieske iron-sulfur protein Rip1 and cytochrome $c_{1}$ (Cyt1) the catalytic redox center of the cytochrome $b c_{1}$ complex (Ndi et al., 2018). These core subunits are surrounded by the additional nuclear encoded proteins Cor1, Cor2, Qcr6, Qcr7, Qcr9 and Qcr10 in yeast (Mileykovskaya et al., 2012; Hartley et al., 2019). Complex III assembly is suggested to start with insertion of cytochrome $b$ into the IMM and the other nuclear subunits are incorporated with the help of numerous assembly factors (Ndi et al., 2018). The complex exists only as a homodimer under in vivo conditions. The dimerization is reported to occur during early assembly independent from late-stage assembly proteins like Rip1 and Qcr10, whereas integration of the core subunits Cor1 and Cor2 appear to be crucial (Conte et al., 2015; Stephan and Ott, 2020). Mature complex $\mathrm{III}_{2}$ contributes to the proton gradient via the two-step Q-cycle (Mitchell, 1976). To this end, electrons derived from ubiquinol (reduced state of coenzyme Q) oxidation travel through complex III by reducing the active centers of cytochrome $b$ and $c_{1}$ (heme) and Rip1 (Fe-S cluster) while releasing protons to the IMS side. Terminally, cytochrome $c_{1}$ (Cyt1) reduces cytochrome $c$ (Cyc1) in order to transfer the electrons to complex IV (reviewed in Ndi et al., 2018; Zhao et al., 2019). Co-enzyme Q represents a two-electron donor, whereas the heme-groups of cytochrome $b, c_{1}$ and $c$ are single-electron acceptors. Although this two-step oxidation of ubiquinol increases the efficiency of proton transfer, it involves the possible danger of reactive oxygen species (ROS) production. It appears that the Q-cycle intermediate ubisemiquinone is a potential source of superoxide anions by reaction with molecular oxygen (reviewed in Turrens, 2003; Brand, 2010).

\subsubsection{Cytochrome c Oxidase - function and biogenesis}

Cytochrome $c$ oxidase consists of three mitochondrially encoded core subunits (Cox1, Cox2, Cox3) plus 9 and 11 additional nuclear encoded structural subunits in yeast and human, respectively (Zong et al., 2018; Hartley et al., 2019). While Cox3 does not contain a catalytic center, Cox 1 and Cox 2 contain various active sites with two heme groups ( $a$ and $a_{3}$ ) and two copper centers $\left(\mathrm{Cu}_{\mathrm{A}}\right.$ and $\left.\mathrm{Cu}_{\mathrm{B}}\right)$. Cytochrome $c$ binds to Cox2 for electron transmission to Cox1 
where oxygen is reduced in the final step by overall four electrons. The oxygen molecule and required protons for the reduction likely enter the complex through Cox3 while the released energy is employed for additional proton pumping into the intermembrane space (reviewed in Fontanesi et al., 2006). All intermediate products remain tightly bound to the complex in order to prevent insufficient electron transfer and undesired ROS production (Blomberg, 2016).

In contrast to complex III as described above, complex IV harbors several mitochondrial encoded subunits. This results in a more complex assembly that has to be achieved by arranging both mitochondrial encoded and nuclear encoded subunits. It is envisioned that this occurs in a modular way, where first the co-factors are integrated into the active centers and some structural subunits are recruited specifically until the modules COX1, COX2, COX3 are assembled together into mature complex IV (Figure 1-4) (Herrmann and Funes, 2005; Mick, Fox and Rehling, 2011; Barros and McStay, 2020).

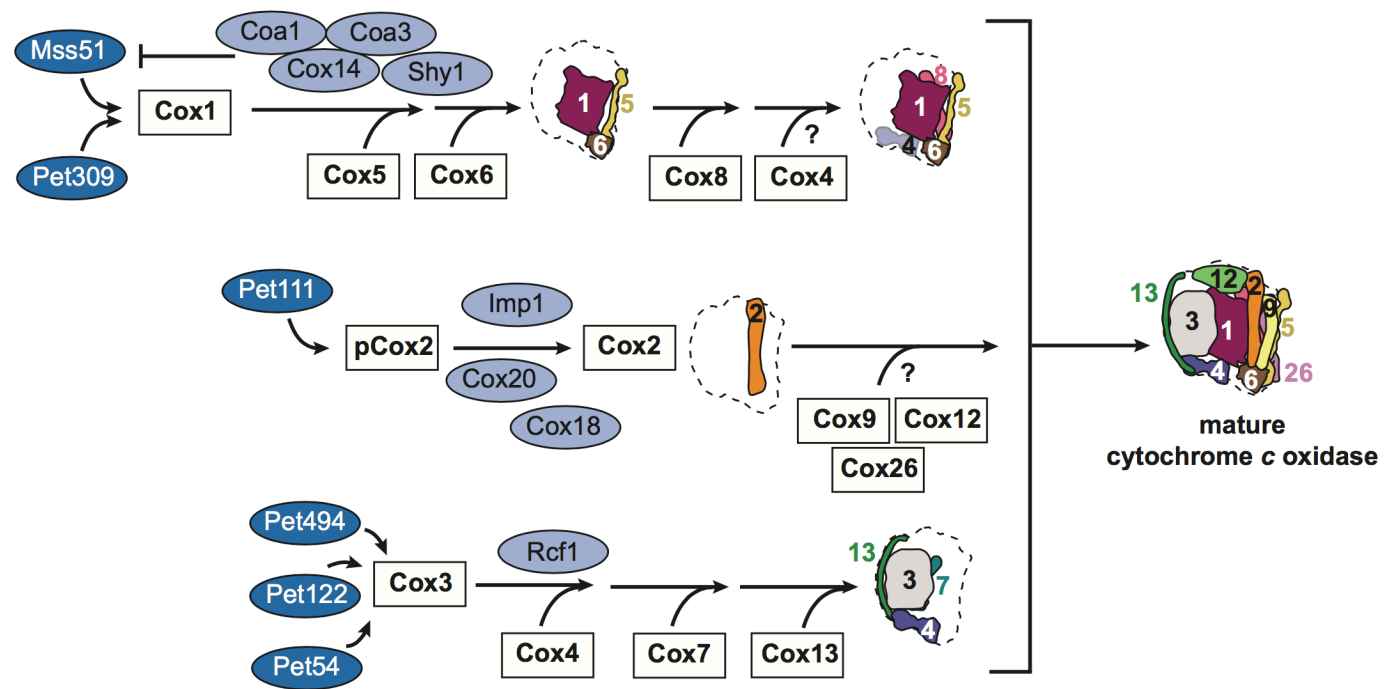

Figure 1-4: Model - overview of yeast cytochrome $c$ oxidase modular assembly.

Transcription of mitochondrial encoded proteins Cox1, Cox2, Cox3 are initiated by various factors depicted in dark blue. Proteins essential for assembly or stabilization of the single lines are depicted in light blue. Structural subunits entering the assembly line are depicted as white boxes. The model represents the modules as they were shown to interact and found in intermediate complexes. ? indicate unsolved but probable assembly lines. The sequence of interactions is not completely resolved and the model does not claim completeness (modified after Mick, Fox and Rehling, 2011; Barros and McStay, 2020).

Mitochondrial translation of yeast Cox1, Cox 2 and $\operatorname{Cox} 3$ can be regulated by different mRNA activators that bind to the respective transcript. Pet309 interacts with Cox1 mRNA, Pet111 with Cox 2 mRNA and Cox3 has three mRNA activators: Pet54, Pet122 and Pet494 (Herrmann and Funes, 2005; Mick, Fox and Rehling, 2011; Barros and McStay, 2020). 
COX1 module. The information we have about the COX1 module is more profound compared to the other two, which can be reasoned by the number of involved factors and intermediate assembly states. This allows a better understanding of the different steps and the sequence of events. Besides Pet309, Mss51 is known to bind with COX1 transcript to control the translation (Decoster et al., 1990; Manthey and McEwen, 1995). Newly synthesized Cox1 is inserted into the IMM by Oxa1 and other insertases where it directly binds to the assembly factors Coa1, Coa3 and Cox14 (Mick et al., 2007; Pierrel et al., 2007; Mick et al., 2010). This complex recruits again Mss51 which remains bound upon further assembly. By this, Mss51 is withdrawn from activating Cox1 translation and is believed to ensure that hemylation and copper insertion only take place when other subunits are present (Barrientos, Zambrano and Tzagoloff, 2004). The intricate procedure of integration of the prosthetic groups is not completely resolved. Several accessory factors were shown to be involved in Cox1 heme maturation and copper insertion (reviewed in Barros and McStay, 2020). Mss51 dissociates from the maturating module to initiate a new cycle of Cox1 translation, whereas Cox14 and Shy1 seem to remain associated (Mick et al., 2007). The first structural subunit to be associated with Cox 1 is Cox $5 \mathrm{a} / \mathrm{b}$. The protein is the only complex IV subunit present in two isoforms and expression depends on the oxygen status of the cell (see also 1.4.4). Cox6 and Cox8 are other structural subunits which are shown to interact within the COX1 module, while Shy1 acts as an assembly factor (Figure 1-4) (Mick et al., 2007; McStay et al., 2013).

COX2 module. Maturation of Cox2 requires proteolytic processing. Upon insertion into the IMM, Cox2 is processed by the inner membrane protease Imp1, while Cox20 and Cox18 act as assisting factors (Tzagoloff et al., 2000; Elliott, Saracco and Fox, 2012). The integration of the binuclear $\mathrm{Cu}_{\mathrm{A}}$ center is achieved by copper chaperones involving Sco1 and Coa6 (Lode et al., 2000; Ghosh et al., 2016). No other structural subunit was found to interact with Cox2 prior interaction with Cox 1 and Cox3 which could also be due to detergent lability reasons. However, Cox12, Cox 26 and Cox 9 could be possible candidates judged by their association in mature complex IV (Figure 1-4) (Franco et al., 2018; Hartley et al., 2019).

COX3 module. Cox3 does not require co-factor insertion since it does not harbor prosthetic groups. The role of Cox3 for complex IV function is not completely resolved since it is not directly involved in electron transport. However, it was reported that it influences the proton uptake, stabilizes complex IV and protects the active sites during oxygen reduction (Tiranti et al., 2000; Gilderson et al., 2003; Varanasi et al., 2006). Several phospholipids are integrated into 
the structure of Cox3, suggested to promote the influence on Cox1 (Tsukihara et al., 1996; Shinzawa-Itoh et al., 2007; Hartley et al., 2019).

Furthermore, Cox 3 was shown to interact with the structural subunits $\operatorname{Cox} 4, \operatorname{Cox} 7$ and $\operatorname{Cox} 13$ prior to complex IV assembly (Su, McStay and Tzagoloff, 2014). Rcf1, first connected with supercomplex assembly (see also section 1.5) seem to play a role in Cox3 module maturation since it interacts with newly synthesized Cox3 (Figure 1-4) (Chen et al., 2012; Strogolova et al., 2012). Although not essential for complex IV biogenesis, it seem to facilitate the modular assembly and acts as a lipid chaperone for Cox3 (Chen et al., 2012; Vukotic et al., 2012; Strogolova et al., 2019). However, the sequence of subunit associations as it could be shown for Cox1 module is still elusive.

Overall, the final association and chronology of module association is not resolved completely. It was hypothesized that the single modules maturate in separate pathways until they merge to holo-complex IV. However, the structural subunit Cox 4 was discovered to interact also with Cox1 in early stages of complex IV maturation (Mick et al., 2007; Su, McStay and Tzagoloff, 2014). Other structural subunits like Cox9, Cox12 and Cox26 (Hartley et al., 2019) in contrast, could not be proven to associate with a specific module prior to maturation. It was be envisioned that Cox1 is the first module to be assembled due to undergoing less frequent turnover than Cox2 or Cox3 (McStay, Su and Tzagoloff, 2013).

\subsubsection{Cytochrome c oxidase - oxygen sensitive subunits}

As mentioned before, the structural subunit Cox 5 exists in two iso-forms. Expressed from two different genes, both proteins possess $66 \%$ similarity (Cumsky et al., 1987). Their expression level depends on the oxygen status of the cell - while Cox $5 \mathrm{a}$ is the predominant isoform under normoxia, Cox5b is expressed under hypoxia (Hodge et al., 1989; Zitomer, Carrico and Deckert, 1997; Fontanesi et al., 2006). Similarly, the mammalian homologs Cox4i-1 and Cox4i-2 are regulated inversely by the availability of oxygen (Fukuda et al., 2007). Although both homologs share this characteristic expression control, the cellular mechanisms of sensing oxygen differ fundamentally (reviewed in Trendeleva, Aliverdieva and Zvyagilskaya, 2014).

Mammalian cells process the information of low oxygen supply by the specific hypoxia inducible factor HIF. Adaptation to hypoxia is mediated post-translationally on the level of HIF protein stability (Semenza, 2007). The factor represents a heterodimer of oxygen sensitive HIF- $\alpha$ and constitutive HIF- $\beta$ subunits. While HIF- $\alpha$ is degraded rapidly under normoxia, the responsible ubiquitin-proteasome is restrained under hypoxia. This leads to accumulation of HIF that 
subsequently translocates into the nucleus and upregulates respective hypoxia specific genes. In parallel it is suggested that the protease LON helps with Cox4i-1 degradation to facilitate the subunit exchange (Fukuda et al., 2007). In contrast, yeast oxygen sensing utilizes the indirect and oxygen dependent pathway of heme biosynthesis. Under aerobic conditions, accumulated heme activates the transcription factors Hap1 and supposedly Hap2/3/4/5. The Hap2/3/4/5 complex promotes COX5a transcription while Hap1 acts on ROX1 transcription. Rox1, in turn, is a transcriptional repressor of COX5b resulting in Cox5a being the prevalent isoform. In case of dropping oxygen levels, the Hap proteins are not active due to restricted heme synthesis. Consequently, COX5b transcription is de-repressed and its protein isoform dominates (Zitomer, Carrico and Deckert, 1997; Kwast, Burke and Poyton, 1998; Trendeleva, Aliverdieva and Zvyagilskaya, 2014). Other proteins connected with the respiratory chain that have a hypoxic counterpart and are controlled similarly, are the electron carrier cytochrome $c$ Cyc1/Cyc7 and the ADP/ATP carrier AAC2/AAC1/AAC3 (Zitomer, Carrico and Deckert, 1997).

Interestingly, Cox 5a/b (and Cox4i-1/Cox4i-2) were suggested to be the first structural subunit interacting with Cox1 (Fontanesi et al., 2006; Mick et al., 2007; McStay et al., 2013; RichterDennerlein et al., 2016). Thus, subunit exchange could occur at a very early stage of complex IV maturation. At the same time, recent studies showed, that Cox $5 \mathrm{a} / \mathrm{b}$ provide an essential physical link of complex IV to complex $\mathrm{III}_{2}$ in the supercomplex (see also section 1.5.1) (Hartley et al., 2019, 2020; Rathore et al., 2019).

\subsection{Respiratory supercomplexes}

In today's studies the association of the respiratory chain as supercomplexes is a well-accepted and thoroughly proven model. However, two opposing theories were first proposed for the state of respiratory complexes: the "solid state" and "fluid state" model (Lenaz and Genova, 2007). According to the "solid state" model, the respiratory complexes associate into single supramolecular units. In parallel, co-enzyme Q and cytochrome $c$ act as electron shuttles within one unit and do not exchange with other complex units (Chance and Williams, 1955; reviewed in Lenaz and Genova, 2007). In contrast to this stands the "fluid state" model where the single complexes are diffusing free and independent from each other. Thus, electron transfer would be coupled to diffusion kinetics while the redox reactions occur upon random collision. This was based on the finding that mammalian co-enzyme Q and cytochrome $c$ show pool behavior 
and the kinetics are saturated at certain concentrations of the electron shuttles (reviewed in Hackenbrock, Chazotte and Gupte, 1986; Milenkovic et al., 2017).

Schägger and Pfeiffer (2000) then revealed biochemically that the respiratory chain of yeast and bovine mitochondria are arranged in supercomplexes. They established a protocol for resolving the association of respiratory supercomplexes by blue-native polyacrylamide electrophoresis (BN-PAGE). In this way, interactions between the complexes remain preserved which represented a fundamental leap in investigating the membrane embedded respiratory complexes. In particular, they showed that yeast complex IV was interacting almost entirely with complex $\mathrm{III}_{2}$ building a complex with one or two complex IV copies: $\mathrm{III}_{2} \mathrm{IV}$ and $\mathrm{III}_{2} \mathrm{IV}_{2}$ (Schägger and Pfeiffer, 2000; Stuart et al., 2000). Analyzing bovine mitochondria, they could show that complex I was predominantly present in a supercomplexed state with complex $\mathrm{III}_{2}$ $\left(\mathrm{I}-\mathrm{III}_{2}\right)$ and most complex IV was associated within I-III ${ }_{2}-\mathrm{IV}_{\mathrm{n}}$ complexes (Schägger and Pfeiffer, 2000). Plenty of studies followed, showing similar results in different organisms (Schägger and Pfeiffer, 2001; Eubel, Jänsch and Braun, 2003; Stroh et al., 2004). These findings pointed towards the "solid" model to be true, although it was under debate if the isolation of supercomplexes is an artefact of using detergents and results in aggregation of the complexes. Consequently, it was a final evidence for in vivo existing supercomplexes, when cryo-tomography of both bovine and fungi mitochondria showed respiratory complexes being associated to supramolecular structures in a detergent free environment (Davies et al., 2018).

Along this line, earlier published in-gel activity assays could prove that the supercomplexes represent active entities but also the free and non-assembled complexes were active as shown for complex IV in yeast (Acín-Pérez et al., 2008a). They also provided evidence for genuine supercomplex assembly by radioactive pulse labeling of mitochondrial encoded subunits followed by different chase timepoints. In the end, this data led to the suggestion of the "plasticity" model that imagines the respiratory chain complexes being present as both, single complexes but also supercomplexes - in a highly dynamic manner (Acín-Pérez and Enriquez, 2014). By this, the two initially contradictory models were unified into one model while still appreciating the biochemical proof for the one and the other. In fact, it was experienced that the respiratory chain complexes respond to a higher demand of energy. Greggio et al. (2017) and Huertas et al., (2017) could show that the amounts of supercomplexes are increased in human and rat after exercise, respectively. This provides strong evidence that the respiratory chain is able to adapt to metabolic requirements in a very flexible way by building supercomplexes. 


\subsubsection{Structure of respiratory supercomplexes}

In the recent years of the $21^{\text {st }}$ century, various studies were focusing on the structure of the respiratory supercomplexes. Remarkable 3D cryo-electron microscopy (EM) data, often combined with embedding X-ray structures, were published in steadily increasing resolution. Structures of yeast supercomplexes $\mathrm{III}_{2} \mathrm{IV}_{(1-2)}$ and mammalian $\mathrm{I}-\mathrm{III}_{2}-\mathrm{IV}$ revealed the arrangement of the single complexes within the supramolecular association (Althoff et al., 2011; Dudkina et al., 2011; Mileykovskaya et al., 2012; Letts, Fiedorczuk and Sazanov, 2016). Even higher molecular weight complexes, so-called megacomplexes, of human mitochondria have been obtained (Guo et al., 2017). While complex I appears to serve as a possible scaffold for association with complex $\mathrm{III}_{2}$ and complex IV, it is lacking in yeast respiratory chain. Still, the singular NADH-Dehydrogenase (Ndi1) was found to interact with complex III, proposing a close association with the yeast supercomplex (Matus-Ortega et al., 2015; Linden et al., 2020).

It was puzzling when Mileykovskaya et al. (2012) resolved the first 3D cryo-EM structure (24 resolution) of yeast supercomplex $\mathrm{III}_{2} \mathrm{IV}_{2}$ which showed a substantially different interacting site of complex IV than previous studies of bovine respirasome (Althoff et al., 2011; Dudkina et al., 2011) and 2D analysis of yeast supercomplexes (Heinemeyer et al., 2007). Bovine complex IV was demonstrated to interact via its convex shaped side via COX6A (yeast Cox13) with complex III (Figure 1-5A) (Althoff et al., 2011; Dudkina et al., 2011; Letts, Fiedorczuk and Sazanov, 2016; Guo et al., 2017), and yeast complex IV appeared to associate via the opposite side, pointing towards an interaction between Cox5a/b and complex III (Figure 1-5B) (Mileykovskaya et al., 2012). Cryo-EM structures with considerably improved resolution of yeast supercomplex $\mathrm{III}_{2} \mathrm{IV}_{(1-2)}(3.3-3.5 \AA)$ provided the evidence that Cox5a is in direct contact with complex III subunits Cor1, Qcr6 and Cyt1 within the mitochondrial matrix and IMS, respectively (Hartley et al., 2019; Rathore et al., 2019). Furthermore, the data showed that Cox5a forms a conjunction to Rip1 and Qcr8 via cardiolipin within the IMM (Hartley et al., 2019). Considering that complex IV subunit Cox 5 can be present in two forms (see also 1.4.4), they eliminated the possibility of mixed supercomplex populations by investigating the $\operatorname{cox} 5 \mathrm{b \Delta}$ strain. The subsequent study of Cox5b-containing supercomplexes displayed once more that the interface between complex IV and $\mathrm{III}_{2}$ is conserved between the two subunits (Hartley et al., 2020). It was suggested that the difference between mammalian and yeast complex IV orientation relative to complex $\mathrm{III}_{2}$ depends on the presence of complex I. In fact, the conserved interacting site of complex III is occupied by complex I in mammalian structures, absent in yeast S. cerevisiae (Rathore et al., 2019). This could be indicative for advantageous channeling of 
coenzyme Q bringing the catalytic centers of complex I and III in closer proximity to each other. At the same time, yeast cytochrome $c$ appears to have a reduced electron transport length from complex III binding site of Cyt1 to the designated complex IV binding site of Cox2. Consequently, it was argued that the respiratory chain functionally adapted to more favorable arrangements in terms of efficient substrate usage (Rathore et al., 2019).

A

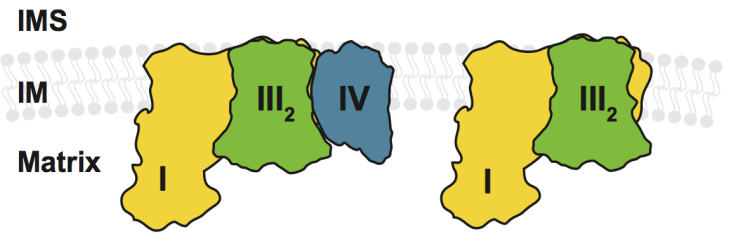

B

IMS

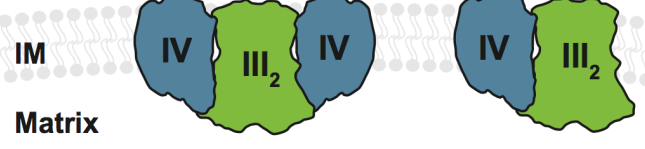

Figure 1-5: Model - structure of mammalian and yeast respiratory chain complexes.

A) The mammalian respiratory chain complexes associate to supramolecular structures, the most abundant complexes are complex $\mathrm{I}_{-} \mathrm{III}_{2}$ and $\mathrm{I}-\mathrm{III}_{2}-\mathrm{IV}$, the so-called respirasome. Complex IV associates via its convex side with complex III (Althoff $e t$ al., 2011; Dudkina et al., 2011). B) Yeast respiratory chain complexes associate to the supercomplexes $\mathrm{III}_{2} \mathrm{IV}_{2}$ and $\mathrm{III}_{2} \mathrm{IV}$ with the convex site of complex IV opposing the interaction side with complex III (Mileykovskaya et al., 2012; Hartley et al., 2019; Rathore et al., 2019).

\subsubsection{Function and biogenesis of respiratory supercomplexes}

To date, a plethora of studies provided valuable information about the existence and arrangement of the respiratory supercomplexes in various organisms and tissues (Schägger and Pfeiffer, 2000; Eubel, Jänsch and Braun, 2003; Reifschneider et al., 2006). However, a functional relevance could only be speculated. It was postulated that they could serve the purpose of substrate channeling since cryo-structures resolved cytochrome $c$ bound to complex IV (Althoff et al., 2011; Mileykovskaya et al., 2012). It was argued that the respective active sites of complex I, III and IV which bind the electron shuttles cytochrome $c$ and coenzyme Q are still too distant and allow free diffusion of the carriers (Althoff et al., 2011; Dudkina et al., 2011; Rathore et al., 2019). Furthermore, studies demonstrated a certain interdependency of single complex stability as shown for patient mutations affecting complex III or complex IV (AcínPérez et al., 2004; Li et al., 2007). This could indicate that supercomplex assembly sustains the single respiratory complexes and might even facilitate the particular assembly as Moreno-Lastres et al. (2012) proposed for complex I.

Due to the spatial proximity of active sites, it was also suggested that supercomplexes decrease the undesired release of ROS. As supported by a study of Maranzana et al. (2013), the supercomplex $\mathrm{I}_{-} \mathrm{III}_{2}$ protects from ROS production originating from complex I. Since complex I is absent in yeast, it might be a different situation in terms of ROS production. Recent 
data from Berndtsson et al. (2020) addressed the same proposition by specific disruption of yeast respiratory supercomplexes $\mathrm{III}_{2} \mathrm{IV}_{(1-2)}$ by mutating the interacting protein Cor1. They could show that ROS production was not significantly different when the complexes were not interconnected. Yet, they provided valid evidence that the arrangement of supercomplexes offers kinetic advantage for the electron transfer and respirational activity is significantly decreased upon supercomplex disruption (Berndtsson et al., 2020). The increased amounts of supercomplexes measured after exercise further support the suggestion that this association results in a more efficient respiration and hence energy production (Greggio et al., 2017; Huertas et al., 2017).

Besides supercomplex function, the mechanism behind supercomplex biogenesis is still obscure. It is under debate if there are single assembly lines of the complexes, first associating to holo-complexes followed by the assembly into supercomplexes (Acín-Pérez et al., 2008b; Moreno-Lastres et al., 2012; Guerrero-Castillo et al., 2017; Protasoni et al., 2020). This was supported by the above mentioned labeling experiments of Acín-Pérez et al. (2008) where they determined a time lag between complex and subsequent supercomplex assembly. Similar results were conducted when following complex I assembly after reversed treatment of inhibiting mitochondrial translation by chloramphenicol (Guerrero-Castillo et al., 2017). Moreno-Lastres et al. (2012) however, could show that complex I maturation occurs after supercomplex formation. This is further supported by data implying a specific role for complex $\mathrm{III}_{2}$ as a starting point for supercomplex assembly while serving as a platform for complex I and complex IV maturation. And this in turn, would point to the supercomplexes as important for overall respiratory chain complex biogenesis (Protasoni et al., 2020).

\subsubsection{Respiratory supercomplexes - assembly and stabilizing factors}

Although the role and function of the respiratory supercomplexes remains ill-defined, all theories have in common that they indicate an essential role in proper mitochondrial function. Given the high consistency in particles obtained by cryo-EM in different laboratories (Althoff et al., 2011; Dudkina et al., 2011; Mileykovskaya et al., 2012; Hartley et al., 2019, 2020; Rathore et al., 2019) and therefore largely uniform population of supercomplexes, it seems obvious that the coordination underlies a specific mechanism. Yet, not completely resolved, several proteins/factors were found to be involved in supercomplex assembly additionally considering that the organization might be different from yeast to mammals. 
Cardiolipin. The inner mitochondrial membrane is mainly composed of the lipids phosphatidylcholine, phosphatidylethanolamine and cardiolipin. The latter is found exclusively in mitochondria and was proven to be a key player in supercomplex stabilization (Zinser et al., 1991; Schenkel and Bakovic, 2014; Rappocciolo and Stiban, 2019).

However, it is discussed if cardiolipin is only responsible for supercomplex stabilization or if it takes part in the formation itself (Pfeiffer et al., 2003; Bazán et al., 2013). Several cryo-EM structures demonstrated that cardiolipin is integrated into yeast supercomplex $\operatorname{III}_{2} \mathrm{IV}_{(1-2)}$ and recently it was even resolved at the interaction site between complex IV subunit Cox 5 and complex III subunits Rip1 and Qcr8 (Mileykovskaya et al., 2012; Hartley et al., 2019, 2020; Rathore et al., 2019). Furthermore, the right balance between cardiolipin and phosphatidylethanolamine with the inner mitochondrial membrane appears to be important since they showed opposing features. Absence of phosphatidylethanolamine leads to a more stable supercomplex in yeast but causes similar defects in respiration and membrane potential (Böttinger et al., 2012). Defects in human cardiolipin synthesis cause heavily compromised mitochondrial structures and manifests in a disease called Barth syndrome. Patients with Barth syndrome suffer from multi-system disorder, first characterized as cardiac disease, emphasizing once more the physiological importance of supercomplex stability (reviewed in Clarke et al., 2013).

SCAF1 (COX7A2L). SCAF1 is expressed in higher eukaryotes and has no yeast homolog. It was first identified due to its high sequence similarity to COX7 isoforms, therefore the name COX7A2L (Lapuente-Brun et al., 2013). Further investigations characterized it as a possible assembly factor of supercomplexes containing complex III and complex IV, since it was only present in those but not the single complexes (reviewed in Lobo-Jarne and Ugalde, 2018). Although initially reported as stabilizing complex IV at the site of complex I (Ikeda et al., 2013; Lapuente-Brun et al., 2013), other studies argued that it is not essential for respirasome $\left(\mathrm{I}-\mathrm{III}_{2}-\mathrm{IV}_{\mathrm{n}}\right.$ ) assembly (Pérez-Pérez et al., 2016). Only the association of complex III and complex IV appears to be affected. Lobo-Jarne et al. (2018) suggested that SCAF1 is involved in a check-point step of complex III assembly and furthermore demonstrated that knock-out cells did not suffer from dysfunctional respirasomes but delayed assembly. This lines up with various observations with mouse models expressing the putative non-functional shorter isoform (Mourier et al., 2014; Davoudi et al., 2016). Although this questions the physiological relevance for complex $\mathrm{III}_{2} \mathrm{IV}_{(1-2)}$, it indicates that complex $\mathrm{I}$ is able to serve as a scaffold in the respirasome 
but that the association between complex III and complex IV is favorable in terms of efficient supercomplex assembly and metabolic fitness (García-Poyatos et al., 2020).

Coi1. Coi1 is conserved among fungi and was identified by Singhal et al. (2017) as a transient interactor of complex IV. Mutant strains display defective supercomplex assembly while heme insertion into Cox1 appears to be affected in parallel. Coi1 does not directly bind to heme or Cox1, yet, it seems to facilitate heme incorporation into complex IV (Singhal et al., 2017). Consequently, it is elusive if supercomplex assembly is directly affected. However, several complex III and complex IV subunits and the Rcf-proteins (see below) were found in its interaction spectrum indicating an involvement in supercomplex assembly and/or stabilization (Singhal et al., 2017).

Rcf-proteins. Rcf1, Rcf2 and Rcf3 share homologous sequences among each other and were characterized as possible assembly factors of respiratory supercomplexes. All three proteins are independently interacting with complex III and complex IV, while predominantly associating via the supercomplex $\mathrm{III}_{2} \mathrm{IV}_{(1-2)}$ in a substoichiometric manner (Chen et al., 2012; Strogolova et al., 2012; Vukotic et al., 2012; Römpler et al., 2016). Rcf1 appears to be involved in Cox3 modular assembly, however, it remains associated at the supercomplex and facilitates Cox13 and Rcf2 association (Strogolova et al., 2012; Vukotic et al., 2012; Garlich et al., 2017). While supercomplex formation is not completely abolished in $r f f 1 \Delta$ but strongly affected, the idea of a true assembly factor was questioned (Chen et al., 2012; Vukotic et al., 2012; Strogolova et al., 2019; Dawitz et al., 2020). Rcf2 and Rcf3 in turn, were demonstrated to have overlapping roles in respect of complex IV regulation (Römpler et al., 2016). It was intriguing when Rcf2 was resolved in a recent cryo-EM structure of allegedly fully assembled hypoxic supercomplex $\mathrm{III}_{2} \mathrm{IV}$ arguing for a stoichiometric interaction under these conditions (Hartley et al., 2020).

Rcf2 and Rcf3 are conserved among fungi, whereas Rcf1 possesses two mammalian homologs the hypoxia inducible HIGD1A and the constitutively expressed HIGD2A (Timón-Gómez et al., 2020b). HIGD2A was first reported as the functional homolog of Rcf1 and appears to be involved in COX3 module maturation and its assembly into complex IV (Chen et al., 2012; Vukotic et al., 2012; Hock et al., 2020). Nevertheless, Timón-Gómez et al. (2020a) recently found HIGD2A to be involved in supercomplex assembly, while it displays overlapping functions with HIGD1A in complex IV biogenesis. HIGD1A in turn, was demonstrated to play an additional role in complex $\mathrm{III}_{2}$ biogenesis (Timón-Gómez et al., 2020a). 


\subsection{Aims of this study}

Efficient mitochondrial respiration is the basis for generating a membrane potential among the inner mitochondrial membrane and by this, for a vast amount of mitochondrial operations, vital for the cell. The discovery of the arrangement as higher molecular respiratory supercomplexes (Schägger and Pfeiffer, 2000) was revolutionary and resulted in various attempts to explain their function. It appears that already small imbalances can cause e.g. dysfunctional respiration, uncontrolled ROS production, underlining the importance for proper regulation. In light of the plasticity model, supercomplex formation is a highly dynamic process, adapting to cellular demands by continuous change between single complexes and supramolecular structures (AcínPérez and Enriquez, 2014). For this, biogenesis and organization of these associations are crucial analyses that have to be accomplished. Since there is a sophisticated interplay and dependency between mammalian complex I and complexes III and IV (Moreno-Lastres et al., 2012; Milenkovic et al., 2017; Lobo-Jarne and Ugalde, 2018), the yeast model system, lacking multimeric complex I, represents a perfect starting point for insights into the association of complex III and IV.

Proteins which were demonstrated to be involved in yeast supercomplex assembly are the Rcf-protein family. While Rcf1 facilitates supercomplex assembly, Rcf2 and Rcf3 were reported to rather play a regulatory role (Chen et al., 2012; Vukotic et al., 2012; Römpler et al., 2016). Besides the interaction of Rcf1 with newly synthesized Cox3 (Strogolova et al., 2012; Garlich et al., 2017), the interacting sites of the Rcf-proteins were unknown at the beginning of this study. One major aim of this work was to identify the localization of the proteins within the respiratory chain in order to conclude interactors and specify their function. During this localization analysis, a so far uncharacterized protein Min8 (YPR010C-A) emerged as associated and was investigated for a basic characterization and influence on the respiratory chain. A cryo-EM structure, published in parallel (Hartley et al., 2020), revealed Rcf2 as a peripheral interactor of hypoxic supercomplex $\mathrm{III}_{2} \mathrm{IV}$ and helped us to validate our established data. In context of an oxygen dependent subunit exchange within complex IV, a potential involvement of Rcf2 was studied more in-depth. Additionally, the feature of the Rcf-proteins sharing partly homologous sequences, let us observe the respective properties of their domains. By constructing several fusion proteins originated from the Rcf domains and thorough analysis in regard of modulation of the supercomplexes, we were aiming for an understanding of the distinct roles of the protein regions.

Conclusively, the Rcf-protein family and their respective domains are evaluated with respect to their role in supercomplex assembly, stabilization and regulation. 


\section{Material and Methods}

\subsection{Material}

All standard chemicals in analytical grade for e.g. buffer preparation were purchased from Merck KGaA (Darmstadt, Germany), Carl Roth (Karlsruhe, Germany), Serva (Heidelberg, Germany) or Sigma Aldrich (now also Merck KGaA; Taufkirchen, Germany). DNA primers were synthesized by and ordered from Microsynth SEQLAB (Göttingen, Germany). Commercial kit systems (Table 2-1) were used as described in the manufacturer's protocol. Special enzymes and reagents used in this study are listed in Table 2-2.

\subsubsection{Kit systems, enzymes and reagents}

Table 2-1: Kit systems used in this study.

\begin{tabular}{ll} 
Kit system & Supplier \\
\hline \hline Fast Digest restriction enzymes & Thermo Fisher Scientific \\
Gene ruler DNA ladder mix 1kb & Thermo Fisher Scientific \\
KOD Hot Start DNA Polymerase & Merck Millipore \\
mMESSAGE mMACHINE ${ }^{\circledR}$ SP6 DNA Transcription Kit & Invitrogen \\
Precision Plus Protein ${ }^{\mathrm{TM}}$ All Blue & Bio-Rad \\
Prestained Protein Standards 10-250 kDa & \\
Rapid Ligation Kit & Thermo Fisher Scientific \\
TNT Flexi Translation & Promega \\
TNT Quick Coupled Transcription/Translation SP6 & Promega \\
Wizard ${ }^{\circledR}$ PLUS SV Minipreps DNA Purification System & Promega \\
Wizard ${ }^{\circledR}$ SV Gel PCR Clean-Up System & Promega \\
& \\
Table 2-2: Special reagents and enzymes used in this study & \\
Reagent/enzyme & Roth \\
\hline \hline -Mercaptoethanol & Supplier \\
${ }^{35}$ S]-Methionine & Roth \\
Acrylamide 4x crystallized & Hartmann Analytic \\
ADP & Roth \\
Agarose NEEO ultra quality & Rothe \\
Ampicillin & Prich \\
\hline
\end{tabular}




\begin{tabular}{|c|c|}
\hline Amplex ${ }^{\mathrm{TM}}$ UltraRed & Invitrogen \\
\hline ANTI-FLAG M2 affinity gel & Sigma Aldrich \\
\hline Antimycin A & Sigma Aldrich \\
\hline APS & Roth \\
\hline Ascorbate & Roth \\
\hline ATP & Roche \\
\hline Bis(sulfosuccinimidyl)suberate $\left(\mathrm{BS}^{3}\right)$ & Thermo Fisher Scientific \\
\hline Bovine serum albumin (BSA), essentially fatty acid free & Sigma Aldrich \\
\hline Bromphenol blue & Merck Millipore \\
\hline CNBr activated sepharose 4B & GE Healthcare \\
\hline Complete supplement mixture & MP biomedicals \\
\hline cOmplete, EDTA free protease inhibitor & Roche \\
\hline Coomassie Brilliant Blue G250 & Roth \\
\hline Coomassie Brilliant Blue R250 & Roth \\
\hline Creatine kinase & Roche \\
\hline Creatine phosphate & Roche \\
\hline Cycloheximide & Sigma Aldrich \\
\hline Digitonin & Merck Millipore \\
\hline Dimethyl pimelidate dihydrochloride (DMP) & Sigma Aldrich \\
\hline DTT & Roth \\
\hline FLAG $^{\circledR}$ peptide & Sigma Aldrich \\
\hline Flexi ${ }^{\circledR}$ Reticulocyte Lysate System & Promega \\
\hline G418 sulphate & GE Healthcare \\
\hline GTP & Sigma Aldrich \\
\hline Herring Sperm DNA & Promega \\
\hline Horse radish peroxidase & Sigma Aldrich \\
\hline IgG from human serum & Sigma Aldrich \\
\hline $\operatorname{IgG}$ protein standard & BioRad \\
\hline Immobilon $^{\circledR}$-P PVDF & Merck Millipore \\
\hline $\mathrm{LiOAc}$ & Applichem \\
\hline Malate & Sigma Aldrich \\
\hline n-Dodecyl $\beta$-D-maltoside (DDM) & Sigma Aldrich \\
\hline N,N'-Methylene-bisacrylamide, $2 \mathrm{x}$ crystallized & Serva \\
\hline NADH & Roche \\
\hline Nourseothricin & Roth \\
\hline
\end{tabular}




\begin{tabular}{ll}
\hline Oligomycin & Sigma Aldrich \\
PEG maleimide, average $\mathrm{M}_{\mathrm{n}} 3350$ & Sigma Aldrich \\
Pierce $^{\circledR} \mathrm{ECL}$ & Thermo Fisher Scientific \\
Pierce $^{\mathrm{TM}}$ Anti-HA Agarose & Thermo Fisher Scientific \\
PMSF & Roth \\
Powdered milk (Frema Reform) & Heirler Cenovis GmbH \\
Protein-A sepharose & GE Healthcare \\
Proteinase K, recombinant & Roche \\
Pyruvate & Sigma Aldrich \\
Röntgenfilm Blau RX-N & Fuji \\
Roti ${ }^{\circledR}$-Quant & Roth \\
Rotiphrese ${ }^{\circledR}$ Gel $30(37.5: 1)$ & Roth \\
Sodium azide & Merck KGaA \\
Succinate & Sigma \\
TEMED & Roth \\
TMPD & Sigma Aldrich \\
Trichloroacetic acid & Roth \\
Valinomycin & Merck Millipore \\
Zymolyase-20T & Nacalai Tesque Inc. (Kyoto, \\
& Japan) \\
\hline
\end{tabular}

\subsubsection{Antibodies}

Polyclonal antibodies were generated in rabbit against $\mathrm{C}$-terminal peptides, recombinant protein domains or recombinant whole protein (Gramsch Laboratories, Schwabhausen, Germany). Commercially purchased monoclonal antibodies and secondary antibodies used in this study are listed below in Table 2-3. Primary antibodies were used in diluted solutions with TRIS buffered saline with $0.1 \%$ Tween 20 (TBS-T) supplemented with $5 \%$ powdered milk. Secondary antibodies were used in a dilution of 1:10 000 in TBS-T.

Table 2-3: Commercially available antibodies used in this study.

\begin{tabular}{ll} 
Antibody & Supplier \\
\hline \hline$\alpha$ FLAG & Sigma Aldrich \\
$\alpha$ MTCO3 (Cox3) & Abcam \\
$\alpha$ HA & Roche \\
Goat $\alpha$ Rabbit HRP & Dianova \\
Goat $\alpha$ Mouse HRP & Dianova
\end{tabular}




\subsubsection{Oligonucleotides and plasmids}

Oligonucleotides used for sequencing, generating gene cassettes for deletion or tagging of proteins, cloning and primers used for generating DNA templates for subsequent transcription and translation were synthesized by Microsynth SEQLAB (Göttingen, Germany) and listed in Table 2-4.

Plasmids used and generated in this study for expression in yeast and as templates for PCR, are listed in Table 2-5. The plasmids carrying the genes for fusion protein expression were generated following the procedures in sections 2.2.2.5 and 2.2.2.6. The corresponding open reading frames were first fused via PCR with overlapping primers in three consecutive PCRs by homologous recombination and then subcloned into the plasmid pRS416.

\subsubsection{Yeast strains}

S. cerevisiae strains used in this study are based on the wild-type strains YPH499 and 777-A3. The genotypes of the wild-type strains and the derivatives used in this study are listed in Table 2-6. Not listed are the respective strains serving as a $U R A^{+}$control carrying the empty plasmid pRS416. This is the case for Wt, $r f 1 \Delta, r c f 2 \Delta r f 3 \Delta, r c f 1 \Delta r f 2 \Delta r c f 3 \Delta, \operatorname{cox} 5 a \Delta, \operatorname{cox} 5 a \Delta r c f 2 \Delta$. 
Table 2-4: Oligonucleotides used in this study.

\begin{tabular}{lll} 
Oligonucleotide Sequence & Target/Function \\
\hline \hline
\end{tabular}

pBH1

$\mathrm{pBH} 2$

$\mathrm{pBH} 3$

pBH4

pBH8

pBH9

pBH10

pBH11

pBH12

pBH13

pBH15

pBH16 $-3$

5'- act aga tgg cat geg tga cat TGT AGT TGA CCG GGA AGA GTT CAA AC -3'

Reverse primer to amplify RCF3 (without Stop), overlapping sequence with RCF1; creating Rcf3-Rcf1

5'- gtt tga act ctt ccc ggt caa cta caA TGT CAC GCA TGC CAT CTA GT -3'

Forward primer to amplify RCF1, overlapping sequence with RCF3 (without Stop); creating Rcf3-Rcf1

5'- tat aga att cCT CGC TTG ACC ATA TAG TAA ATT TG -3'

Reverse primer to amplify RCF1 ( +500 bp downstream)

5'- act aga tgg cat geg tga cat GTA CAT TGT AGC GTC GAA ATT GTT CGA G -3'

Reverse primer to amplify RCF2 $2^{1-83}$, overlapping sequence with RCF1; creating Rcf2 ${ }^{\mathrm{N}}$-Rcf1

5'- CTC GAA CAA TTT CGA CGC TAC AAT GTA Cat gtc acg cat gcc atc tag $\mathrm{t}-3^{\prime}$

Forward primer to amplify RCF1, overlapping sequence with $\mathrm{RCF} 2^{1-83}$; creating Rcf2 ${ }^{\mathrm{N}}$-Rcf1

5'- gtt tga act ctt ccc ggt ca acta caG GAT CCG GTT CCT CCT CGG A - 3'

5'- tcc gag gag gaa cgg gat ccT GTA GTT GAC CGG GAA GAG TTC AAA C -3'

Forward primer to amplify RCF2 ${ }^{84-224}$; creating Rcf3-Rcf2 ${ }^{\mathrm{C}}$$$
\text { 5'- tat ata gtc gac CAT GTA TGT GTA GAT ATG TA -3' }
$$$$
\text { 5'- tat ata gag ctC CCT CGT CGT CCA CTG TTA TA -3' }
$$

5'-tcc gag gag gaa ccg gat ccC ATT GTG CGA TGT TGG TGA GT--3'

5'-act cac caa cat cgc aca atg GGA TCC GGT TCC TCC TCG
Reverse primer to amplify RCF3 without Stop, overlapping sequence with RCF2 ${ }^{84-224}$; creating Rcf3-Rcf2 ${ }^{\mathrm{C}}$

Forward primer for amplifying RCF2 (-500 bp upstream)

Reverse primer for amplifying RCF2 (+500 bp downstream)

Reverse primer to amplify promotor region of RCF2 + ATG, overlapping sequence with $\mathrm{RCF} 2^{84-224}$; creating Rcf2 ${ }^{84-}$ 225 expressed under endogenous promotor

$\begin{array}{ll}\text { GA-3' } & \begin{array}{l}\text { sequence with promotor region of RCF2; creating Rcf2 } \\ \text { expre-225 }\end{array}\end{array}$ 


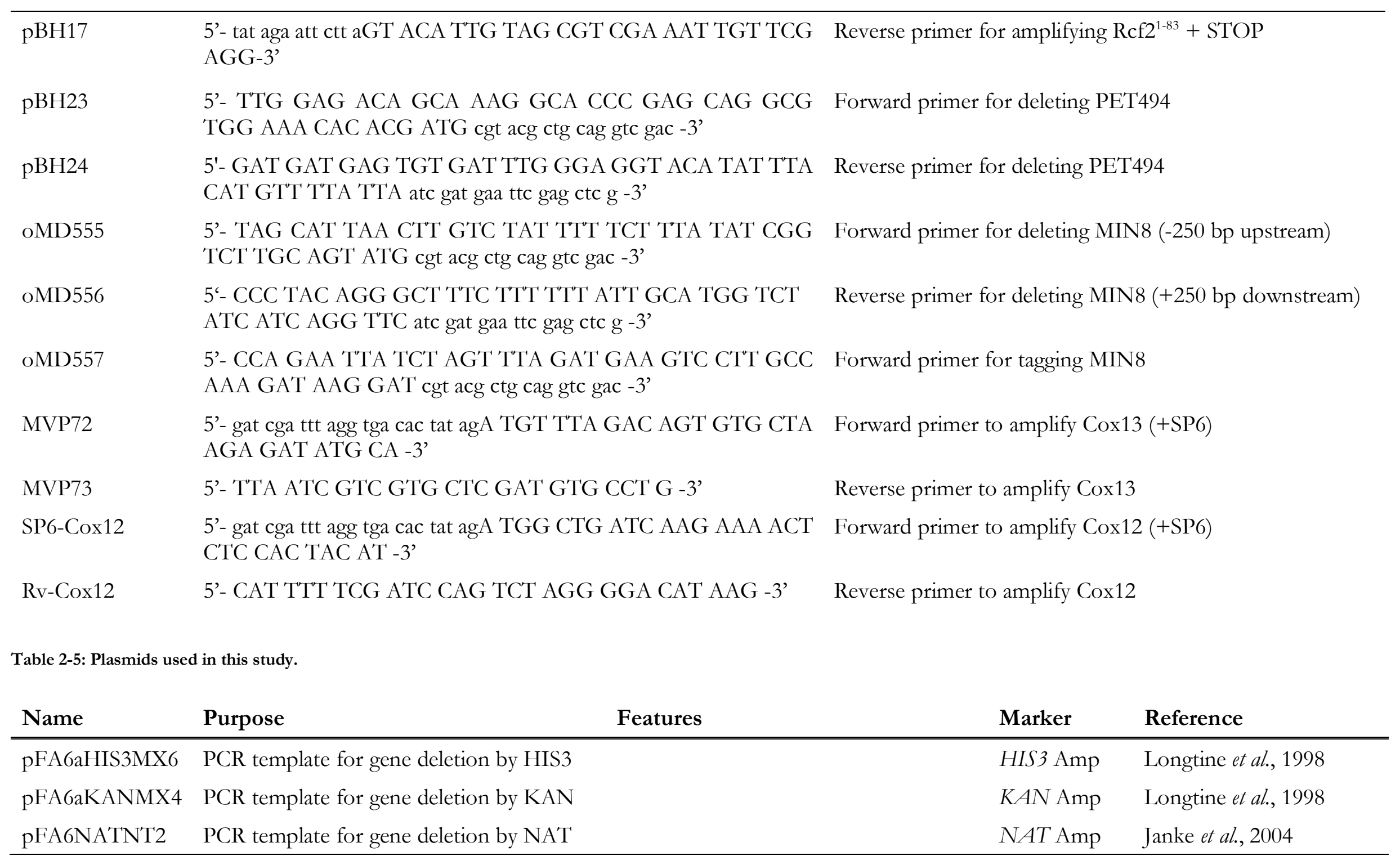




\begin{tabular}{|c|c|c|c|c|}
\hline pRS416 & Yeast plasmid & CEN & URA3 Amp & Sikorski and Hieter, 1989 \\
\hline pYM2 & PCR template for C-terminal 3HA tagging & $3 \mathrm{HA}$ & HIS3 Amp & Janke et al., 2004 \\
\hline${ }^{\mathrm{FLAG}} \mathrm{Rcf} 2$ & $\begin{array}{l}\text { Expression of N-terminally FLAG tagged } \\
\text { Rcf } 2\end{array}$ & ${ }^{\mathrm{FLAG}} \mathrm{Rcf} 2$ in $\mathrm{pRS} 416$ & URA3 Amp & Römpler et al., 2016 \\
\hline Rcf1 & Expression of Rcf1 & $\mathrm{RCF} 1$ orf + promotor in pRS416 & URA3 Amp & This study \\
\hline Rcf2 & Expression of Rcf2 & $\mathrm{RCF} 2$ orf + promotor in pRS416 & URA3 Amp & $\begin{array}{l}\text { Römpler, 2016; } \\
\text { dissertation }\end{array}$ \\
\hline Rcf3-Rcf1 & Expression of Rcf3-Rcf1 & $\begin{array}{l}\text { RCF3 orf }+ \text { promotor }+ \text { RCF1 orf in } \\
\text { pRS416 }\end{array}$ & URA3 Amp & This study \\
\hline Rcf2 ${ }^{\mathrm{N}}-\mathrm{Rcf1}$ & Expression of Rcf2 $2^{1-83}$-Rcf1 & $\begin{array}{l}\mathrm{RCF} 2^{1-83} \text { orf }+ \text { promotor }+ \text { RCF1 orf } \\
\text { in pRS416 }\end{array}$ & URA3 Amp & This study \\
\hline Rcf3-Rcf2 ${ }^{\mathrm{C}}$ & Expression of Rcf3-Rcf2 ${ }^{84-224}$ & $\begin{array}{l}\mathrm{RCF} 3 \text { orf }+ \text { promotor }+\mathrm{RCF}^{84-224} \text { orf } \\
\text { in pRS416 }\end{array}$ & URA3 Amp & This study \\
\hline Strain & Genotype & & \multicolumn{2}{|c|}{ Reference } \\
\hline $777-3 \mathrm{~A}$ & $M A T \alpha$, ade 1 op1 & & \multicolumn{2}{|c|}{ Netter et al., 1982} \\
\hline pet494A & MATa, ade1 op1, pet494::KANMX6 & & \multicolumn{2}{|c|}{ This study } \\
\hline YPH499 & $M A T \alpha$, ade 2-101, his3- $\Delta 200$, leu2- $\Delta 1$, ura3-52, tr & $1-\Delta 63$, lys $2-801$ & \multicolumn{2}{|c|}{ Sikorski \& Hieter, 1989} \\
\hline$r c f 1 \Delta$ & $M A T \alpha$, ade2-101, his3- $\Delta 200$, leu2- $\Delta 1$, ura3-52, tr & 1- $\Delta 63$, lys2-801, rcf1::loxP & \multicolumn{2}{|c|}{ Vukotic et al., 2012} \\
\hline
\end{tabular}




\begin{tabular}{|c|c|c|}
\hline$r c f 2 \Delta$ & $M A T \alpha$, ade2-101, bis3- $\Delta 200$, leu2- $\Delta 1$, ura3-52, trp1- $\Delta 63$, lys2-801, rcf2::HISMX6 & Römpler et al., 2016 \\
\hline$r c f 3 \Delta$ & $M A T \alpha$, ade2-101, bis3- $\Delta 200$, leu2- $\Delta 1$, ura3-52, trp1- $\Delta 63$, lys2-801, rcf3::HISMX6 & Römpler et al., 2016 \\
\hline $\operatorname{rcf} 2 \Delta \operatorname{rcf} 3 \Delta$ & $M A T \alpha$, ade2-101, bis3- $\triangle 200$, leu2- $\Delta 1$, ura3-52, trp1- $\Delta 63$, lys2-801, rcf2::KANMX4, rcf3::HISMX6 & Römpler et al., 2016 \\
\hline$r c f 1 \Delta r c f 2 \Delta r c f 3 \Delta$ & 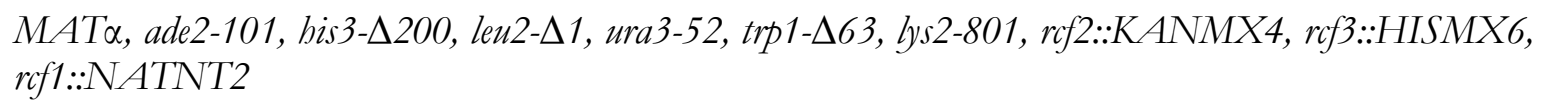 & Römpler, 2016; dissertation \\
\hline $\operatorname{cox} 5 a \Delta$ & 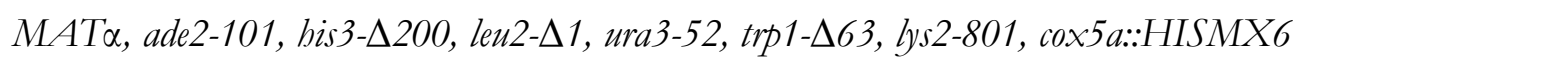 & Römpler et al., 2016 \\
\hline $\operatorname{cox} 5 b \Delta$ & $M A T \alpha$, ade2-101, bis3- $\Delta 200$, leu2- $\Delta 1$, ura3-52, trp1- $\Delta 63$, lys2-801, cox5b::HISMX6 & Rehling group collection \#580 \\
\hline $\operatorname{cox} 5 a \Delta \operatorname{rcf} 2 \Delta$ & 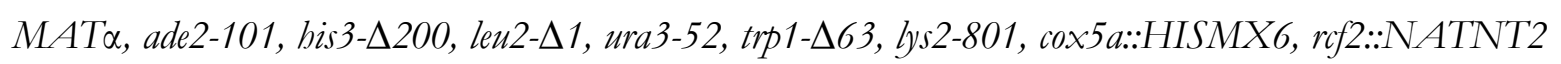 & This study \\
\hline $\min 8 \Delta$ & $M A T \alpha$, ade2-101, his3- $\Delta 200$, leu2- $\Delta 1$, ura3-52, trp1- $\Delta 63$, lys2-801, min8::HISMX6 & Linden et al., 2020 \\
\hline Min8-HA & $M A T \alpha$, ade2-101, his3- $\Delta 200$, leu2- $\Delta 1$, ura3-52, trp1- $\Delta 63$, lys2-801, min8::min8-3HA-HISMX6 & Linden et al., 2020 \\
\hline $\min 8 \Delta \operatorname{rcf} 2 \Delta$ & 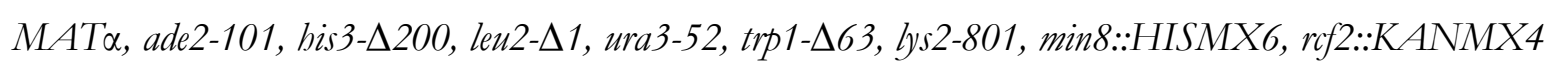 & This study \\
\hline Wt Rcf3-Rcf1 & 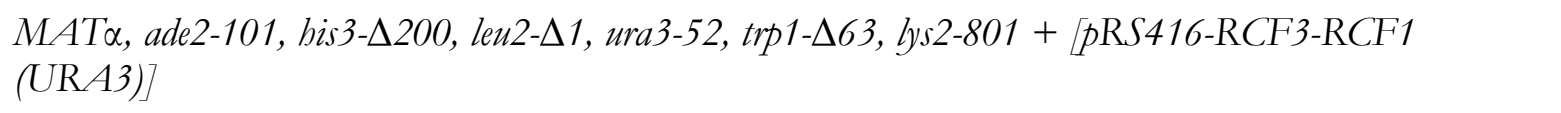 & This study \\
\hline $\begin{array}{l}\text { Wt } \\
\text { Rcf2 } 2^{\mathrm{N}}-\operatorname{Rcf} 1\end{array}$ & $\begin{array}{l}M A T \alpha, \text { ade2-101, bis3- } \Delta 200, \text { leu2- } \Delta 1, \text { ura3-52, trp1- } \Delta 63, \text { lys2-801 }+\left[p R S 416-R C F 2^{N}-R C F 1\right. \\
(U R A 3)]\end{array}$ & This study \\
\hline $\begin{array}{l}\text { Wt } \\
\text { Rcf3-Rcf2 }{ }^{\mathrm{C}}\end{array}$ & $\begin{array}{l}M A T \alpha, \text { ade2-101, bis3- } \Delta 200, \text { leu2- } \Delta 1, \text { ura3-52, trp1- } \Delta 63, \text { lys2-801 }+\left[p R S 416-R C F 3-R C F 2^{C}-\right. \\
(U R A 3)]\end{array}$ & This study \\
\hline $\begin{array}{l}\operatorname{rcf1\Delta } \\
\text { Rcf3-Rcf1 }\end{array}$ & 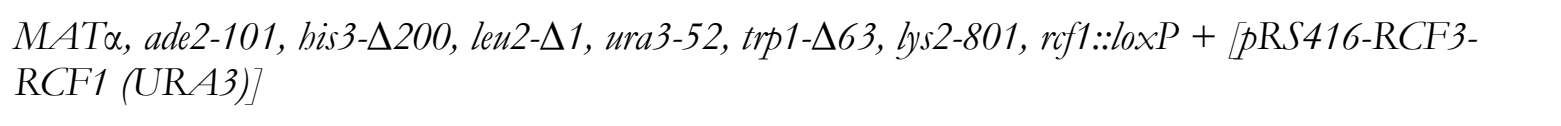 & This study \\
\hline $\begin{array}{l}\operatorname{rcf1} \Delta \\
\operatorname{Rcf} 2^{\mathrm{N}}-\mathrm{Rcf} 1\end{array}$ & 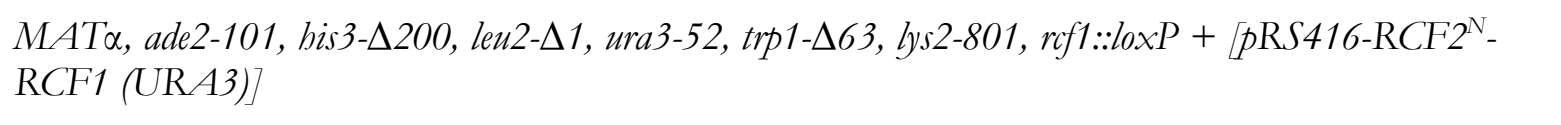 & This study \\
\hline $\begin{array}{l}\operatorname{rcf1\Delta } \\
\operatorname{Rcf3}-\operatorname{Rcf} 2^{\mathrm{C}}\end{array}$ & $\begin{array}{l}M A T \alpha, \text { ade2-101, bis3- } \Delta 200, \text { leu2- } \Delta 1, \text { ura3-52, trp1- } \Delta 63, \text { lys2-801, rff1::loxP + [pRS416-RCF3- } \\
\left.R C F 2^{C}-(U R A 3)\right]\end{array}$ & This study \\
\hline
\end{tabular}




\begin{tabular}{|c|c|c|}
\hline $\begin{array}{l}\operatorname{rcf} 2 \Delta \operatorname{rcf} 3 \Delta \\
\text { Rcf3-Rcf1 }\end{array}$ & 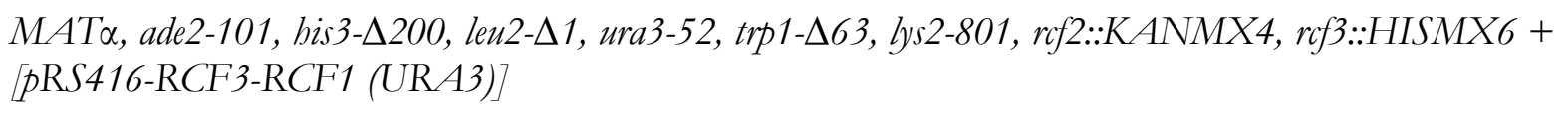 & This stuc \\
\hline$\Delta$ & 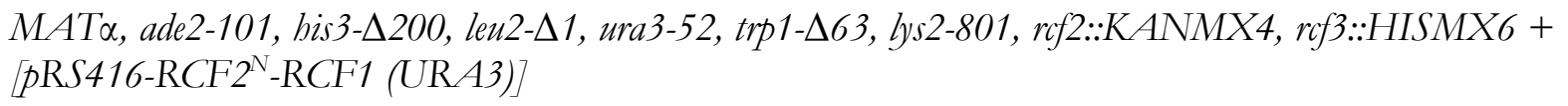 & This \\
\hline $\begin{array}{l}\operatorname{rcf} 2 \Delta r c f 3 \Delta \\
\operatorname{Rcf3}_{-} \operatorname{Rcf}^{\mathrm{C}}\end{array}$ & 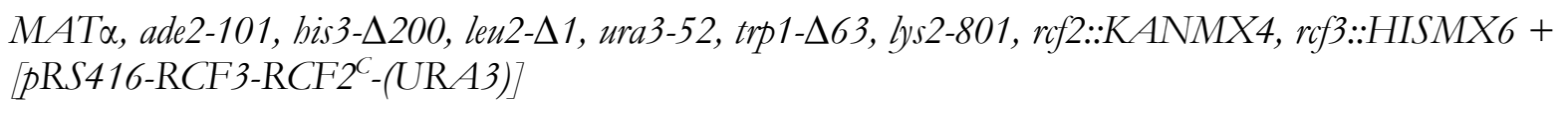 & This \\
\hline $\begin{array}{l}r c f 1 \Delta r \\
\text { Rcf3- }\end{array}$ & 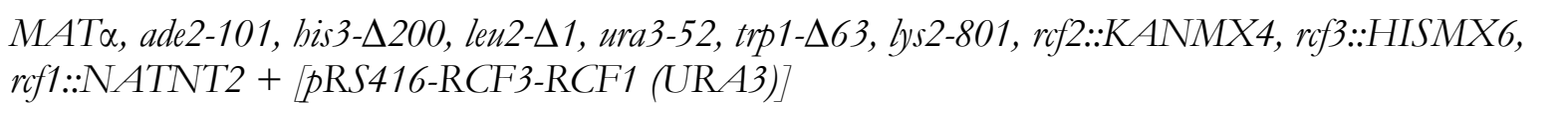 & This \\
\hline $\begin{array}{l}\Delta r c f 3 \Delta \\
\operatorname{cf1}\end{array}$ & 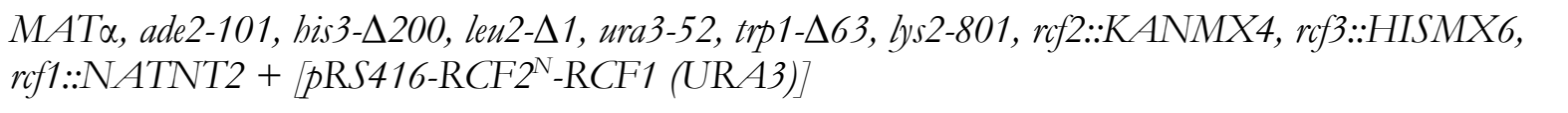 & This \\
\hline $\begin{array}{l}\operatorname{rcf} 1 \Delta r c f 2 \Delta r f 3 \Delta \\
\text { Rcf1 }\end{array}$ & 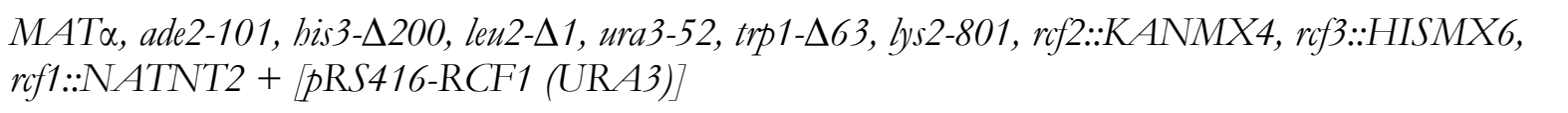 & This \\
\hline $2 \Delta r f 3 \Delta$ & 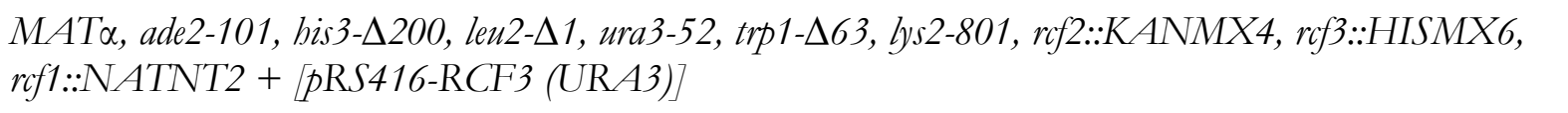 & Thi \\
\hline $\begin{array}{l}\operatorname{rcf} 2 \Delta r c f 3 \Delta \\
\operatorname{Rcf} 2^{C}\end{array}$ & 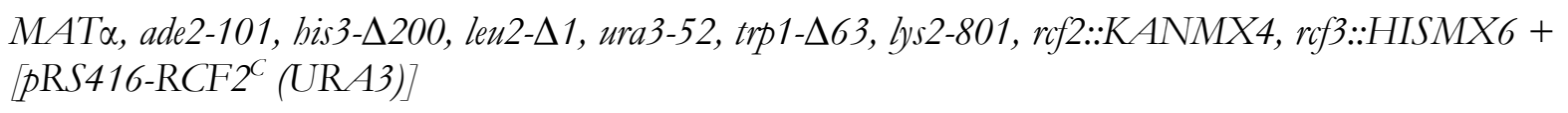 & This study \\
\hline $\begin{array}{l}\operatorname{rcf} 2 \Delta r c f 3 \Delta \\
\operatorname{Rcf} 2^{\mathrm{N}}\end{array}$ & 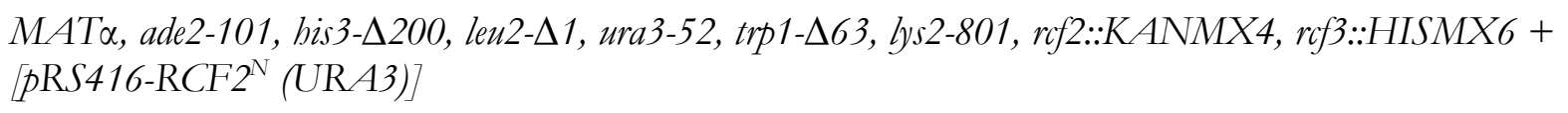 & tudy \\
\hline $\begin{array}{l}\operatorname{cox} 5 a \Delta \\
{ }^{\mathrm{FLAG}} \mathrm{Rcf} 2\end{array}$ & 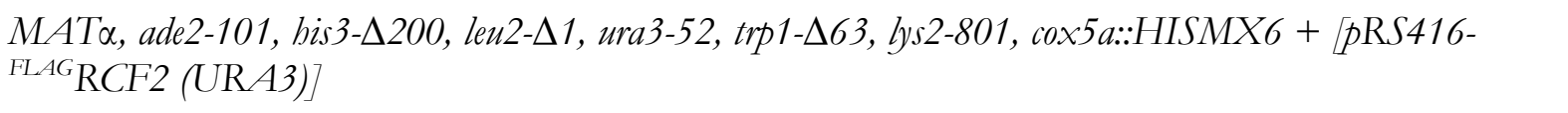 & This study \\
\hline $\begin{array}{l}\operatorname{cox} 5 a \Delta \operatorname{rcf} 2 \Delta \\
\text { FLAGRcf2 }\end{array}$ & $M A T \alpha$, ade2-101, his3- $\triangle 200$, leu2- $\Delta 1$, ura3-52, trp1- 463 , lys2-801, cox5a::HISMX6, rcf2::NATNT2 & This study \\
\hline
\end{tabular}




\subsubsection{Instruments and Software}

Instruments which were utilized for conducting the experiments are listed in Table 2-7. Software for processing the data and finalize this work is listed in Table 2-8.

Table 2-7: Instruments used in this study.

\section{Instrument}

Amersham ${ }^{\text {TM }}$ ImageQuant ${ }^{\text {TM }} 800$

Amersham $^{\text {TM }}$ Typhoon $^{\text {TM }}$ Scanner

Bio Photometer

Centrifuge 5424

Centrifuge 5424R

Centrifuge Avanti J-26 XP

Centrifuge Sorvall Bios16

Curix 60 (developing machine)

JA-10 (rotor)

JA-20 (rotor)

Nanodrop ONE ${ }^{\mathrm{C}}$

Oroboros 2k Oxygraph Series G

Potter S (dounce homogenisator)

SAFAS Xenius XC spectrofluorometer

SE600 Ruby system

Storage Phosphor Screens

Thermomixer comfort

Vacuum Gel dryer 3545

Varian Cary Bio UV-Visible Spectrophotometer

Vortex Genie 2

\section{Manufacturer}

GE Healthcare

GE Healthcare

Eppendorf

Eppendorf

Eppendorf

Beckmann Coulter

Thermo Scientific

AGFA

Beckman Coulter

Beckman Coulter

Thermo Scientific

Oroboros (Innsbruck, Austria)

Sartorius

SAFAS (Monaco)

Hoefer, GE Healthcare

GE Healthcare

Eppendorf

LTF Labortechnik

Varian

Scientific Industries

Table 2-8: Software used in this study

\section{Software}

DatLab Version 6.0

Fiji (ImageJ)

\section{Producer}

Oroboros Instruments

(Innsbruck, Austria)

Open Source; fiji.sc

(Schindelin et al., 2012) 


\begin{tabular}{ll}
\hline Geneious & $\begin{array}{l}\text { Biomatters } \\
\text { (Auckland, New Zealand) } \\
\text { Illustrator CS6 }\end{array}$ \\
Adobe Systems \\
(San Jose CA, USA) \\
ImageQuant TL & $\begin{array}{l}\text { GE Healthcare BioSciences AB } \\
\text { (Uppsala Sweden) }\end{array}$ \\
Microsoft Office 2011 & $\begin{array}{l}\text { Microsoft Corporation } \\
\text { (Redmond USA) }\end{array}$ \\
Photoshop CS6 & $\begin{array}{l}\text { Adobe Systems } \\
\text { (San Jose CA, USA) }\end{array}$ \\
Snapgene Viewer & $\begin{array}{l}\text { Insightful Science } \\
\text { (San Diego CA, USA) }\end{array}$ \\
The PyMOL Molecular Graphics System, & Schrödinger, LLC \\
Version 2.4.2 & (New York NY, USA) \\
Zotero & Open Source; Corporation for \\
& Digitial Scholarship \\
(Vienna VA, USA)
\end{tabular}

\subsubsection{Buffers, Solutions and Media}

Buffers, special solutions and media used for the methods described in section 2.2 are listed below in Table 2-9.

Table 2-9: Buffer and solutions used in this study.

\begin{tabular}{|c|c|}
\hline Buffer/Medium & Ingredients and concentrations \\
\hline Blue-Native anode buffer & 50 mM BIS-TRIS pH 7.0 with $\mathrm{HCl}$ \\
\hline Blue-Native cathode buffer & $50 \mathrm{mM}$ Tricine, $15 \mathrm{mM}$ BIS-TRIS pH 7.0 with $\mathrm{HCl}$ \\
\hline Blue-Native gel buffer $(1 \mathrm{x})$ & $\begin{array}{l}\text { 66.67 mM 6-aminocaproic acid, } 50 \text { mM BIS-TRIS, pH } 7.0 \\
\text { with } \mathrm{HCl}\end{array}$ \\
\hline Blue-Native sample buffer & $\begin{array}{l}5 \% \text { Coomassie Brilliant Blue G250, } \\
500 \text { mM 6-aminocaproic acid, } 100 \text { mM BIS-TRIS, } \\
\text { pH } 7.0 \text { with } \mathrm{HCl}\end{array}$ \\
\hline $\begin{array}{l}\text { Blue-Native solubilization } \\
\text { buffer }\end{array}$ & $\begin{array}{l}20 \mathrm{mM} \text { TRIS pH } 7.4 \text { with } \mathrm{HCl}, 1 \mathrm{mM} \text { EDTA, } 100 \mathrm{mM} \\
\mathrm{NaCl}, 5 \% \text { glycerol and } 1 \mathrm{mM} \text { PMSF }\end{array}$ \\
\hline BN-PAGE resolving gel mix & $\begin{array}{l}\text { 4-16\% acrylamide/bisacrylamide }(49.5 \% / 3 \%), 1 \times \mathrm{BN} \text { gel } \\
\text { buffer (see above), } 0-20 \% \text { Glycerol }\end{array}$ \\
\hline BN-PAGE stacking gel mix & $\begin{array}{l}2 \% \text { acrylamide/bisacrylamide }(49.5 \% / 3 \%), 1 \times \text { BN gel } \\
\text { buffer (see above) }\end{array}$ \\
\hline
\end{tabular}




\begin{tabular}{|c|c|}
\hline $\begin{array}{l}\text { Coomassie Brilliant Blue } \\
\text { destaining solution }\end{array}$ & $10 \%$ HAc, $40 \%$ ethanol \\
\hline $\begin{array}{l}\text { Coomassie Brilliant Blue } \\
\text { staining solution }\end{array}$ & Coomassie Brilliant Blue R250, $10 \%$ HAc, 40 \% ethanol \\
\hline Crosslinking buffer & 20 mM HEPES pH 7.5 with $\mathrm{KOH}, 100 \mathrm{mM} \mathrm{NaCl}$ \\
\hline DTT buffer & 100 mM TRIS pH 9.4 using $\mathrm{H}_{2} \mathrm{SO}_{4}, 10$ mM DTT \\
\hline EM buffer & 10 mM MOPS, 1 mM EDTA, pH 7.2 with KOH \\
\hline Homogenization buffer & $\begin{array}{l}600 \mathrm{mM} \text { sorbitol, } 10 \mathrm{mM} \text { TRIS pH } 7.4 \text { using } \mathrm{HCl} \\
1 \mathrm{mM} \text { EDTA, } 2 \text { g/l BSA, } 1 \text { mM PMSF }\end{array}$ \\
\hline Import buffer & $\begin{array}{l}250 \mathrm{mM} \text { sucrose, } 10 \mathrm{mM} \text { MOPS pH } 7.2 \text { with } \mathrm{KOH}, 80 \mathrm{mM} \\
\mathrm{KCl}, 2 \mathrm{mM} \mathrm{KH} \mathrm{PO}_{4}, 5 \mathrm{mM} \mathrm{MgCl} \text {, } 5 \mathrm{mM} \text { methionine, } 3 \% \\
\text { fatty-acid free BSA }\end{array}$ \\
\hline IP solubilization buffer & $\begin{array}{l}20 \mathrm{mM} \text { TRIS (pH } 7.4 \text { with } \mathrm{HCl}), 80 \mathrm{mM} \mathrm{NaCl}, 0.5 \mathrm{mM} \\
\text { EDTA (pH } 8.0 \text { with } \mathrm{NaOH}), 10 \% \text { glycerol, 1x cOmplete } \\
\text { protease inhibitor cocktail (Roche/Merck), } 1 \mathrm{mM} \text { PMSF }\end{array}$ \\
\hline LB medium & $1 \% \mathrm{NaCl}, 0.5 \%$ yeast extract, $1 \%$ tryptone \\
\hline PBS buffer & $\begin{array}{l}137 \mathrm{mM} \mathrm{NaCl}, 2.7 \mathrm{mM} \mathrm{KCl}, 10 \mathrm{mM} \mathrm{Na} \mathrm{HPO}_{4}, 1.8 \mathrm{mM} \\
\mathrm{KH}_{2} \mathrm{PO}_{4}\end{array}$ \\
\hline PEG buffer & $\begin{array}{l}100 \mathrm{mM} \text { LiOAc, } 10 \mathrm{mM} \text { TRIS pH } 8.0 \text { with HCl, EDTA } \\
\text { pH } 8.0 \text { with } \mathrm{NaOH}, 40 \% \text { PEG3350 }\end{array}$ \\
\hline $\begin{array}{l}\text { Respiration/ROS assay } \\
\text { buffer }\end{array}$ & $\begin{array}{l}225 \mathrm{mM} \text { sucrose, } 75 \mathrm{mM} \text { mannitol, } 10 \mathrm{mM} \text { TRIS pH 7.4, } \\
10 \mathrm{mM} \mathrm{KH}_{2} \mathrm{PO}_{4}, 5 \mathrm{mM} \mathrm{MgCl}_{2}, 10 \mathrm{mM} \mathrm{KCl}\end{array}$ \\
\hline SDS running buffer & 25 mM TRIS, 191 mM glycine, $0.1 \%$ SDS \\
\hline SDS sample buffer $(1 \mathrm{x})$ & $\begin{array}{l}2 \% \text { SDS, } 10 \% \text { glycerol, } 60 \text { mM TRIS pH } 6.8 \text { with } \mathrm{HCl} \text {, } \\
1 \% \beta \text {-mercaptoethanol, } 0.01 \% \text { bromphenol blue }\end{array}$ \\
\hline $\begin{array}{l}\text { SDS-PAGE resolving gel } \\
\text { mix }\end{array}$ & $\begin{array}{l}10-16 \% \text { acrylamide/bisacrylamide }(30 \%, 37.5 / 1), 380 \mathrm{mM} \\
\text { TRIS-HCl pH 8.8, 0.1\% SDS, 0-3.5\% Glycerol, 0-100 mM } \\
\text { sucrose }\end{array}$ \\
\hline SDS-PAGE stacking gel mix & $\begin{array}{l}5 \% \text { acrylamide/bisacrylamide }(37.5 / 1), 80 \mathrm{mM} \text { TRIS-HCl } \\
\text { pH } 6.8,0.1 \% \text { SDS }\end{array}$ \\
\hline SEM buffer & $\begin{array}{l}250 \mathrm{mM} \text { sucrose, } 20 \mathrm{mM} \text { MOPS pH } 7.2 \text { using } \mathrm{HCl} \text {, } \\
1 \mathrm{mM} \text { EDTA }\end{array}$ \\
\hline SORB buffer & $\begin{array}{l}100 \mathrm{mM} \text { LiOAc, } 10 \mathrm{mM} \text { TRIS pH } 8.0 \text { with HCl, EDTA } \\
\text { pH } 8.0 \text { with } \mathrm{NaOH}, 1 \mathrm{M} \text { sorbitol, pH } 8.0 \text { with HAc }\end{array}$ \\
\hline TAE buffer & 40 mM TRIS, 20 mM acetic acid, 1 mM EDTA pH 8.0 \\
\hline TBS-T & $\begin{array}{l}20 \text { mM TRIS pH } 7.5 \text { with } \mathrm{HCl}, 62 \mathrm{mM} \mathrm{NaCl} \text {, } \\
0.1 \% \text { Tween } 20\end{array}$ \\
\hline Transfer buffer & $20 \mathrm{mM}$ TRIS, $150 \mathrm{mM}$ glycine, $0.02 \%$ SDS, $20 \%$ ethanol \\
\hline Translation buffer & $\begin{array}{l}600 \mathrm{mM} \text { sorbitol, } 150 \mathrm{mM} \mathrm{KCl}, 15 \mathrm{mM} \text { potassium } \\
\text { phosphate buffer } \mathrm{pH} \text { 7.4, } 20 \mathrm{mM} \text { TRIS } \mathrm{pH} 7.4 \text { with } \mathrm{HCl} \text {, } \\
3 \mathrm{mg} / \mathrm{mL} \text { BSA, } 4 \mathrm{mM} \text { ATP, } 0.5 \mathrm{mM} \text { GTP, }\end{array}$ \\
\hline
\end{tabular}


$6 \mathrm{mM} \alpha$-ketoglutarate, $8 \mathrm{mM}$ creatine phosphate, $0.1 \mathrm{mM}$ methionine-free amino acid mix, $5 \mu \mathrm{g} / \mathrm{mL}$ cycloheximide, $12,7 \mathrm{mM} \mathrm{MgSO}_{4}, 256 \mu \mathrm{g} / \mathrm{mL}$ creatine kinase

Tricine SDS anode buffer

$0.2 \mathrm{M}$ TRIS pH 8.9 with $\mathrm{HCl}$

Tricine SDS cathode buffer

$0.1 \mathrm{M}$ Tricine, $0.1 \mathrm{M}$ TRIS, $0.1 \%$ SDS, pH 8.25 with $\mathrm{HCl}$

Tricine SDS gel buffer (1x)

1 M TRIS, $0.1 \%$ SDS, pH 8.45

Tricine SDS-PAGE

10-18 \% acrylamide/bisacrylamide (49.5\%/3\%), $1 \mathrm{x}$ Tris-

resolving gel mix

Tricine gel buffer (see above), 0-13\% glycerol

Tricine SDS-PAGE stacking gel mix

$4 \%$ acrylamide/bisacrylamide (49.5\%/3\%), 1x TRIS-

Tricine SDS gel buffer (see below)

Urea buffer (for protein unfolding)

$8 \mathrm{M}$ urea, $30 \mathrm{mM}$ MOPS pH 7.2 with $\mathrm{KOH}, 50 \mathrm{mM}$ DT'T

Urea SDS running buffer

$50 \mathrm{mM}$ Tris, $192 \mathrm{mM}$ glycine and $0.1 \%$ SDS

Urea SDS-PAGE resolving gel mix

$30 \%$ acrylamide/bisacrylamide (60\%/0.8\%), $5.4 \mathrm{M}$ urea, 680 mM TRIS- $\mathrm{HCl}$ pH 8.8, 8 mM NaCl, $0.09 \%$ SDS

Urea SDS-PAGE stacking gel mix

$9 \%$ acrylamide/bisacrylamide (60\%/0.8 \%), 3.6 M urea, $100 \mathrm{mM}$ TRIS-HCl pH 6.8, $0.12 \%$ SDS

YNB medium,

$0.67 \%$ YNB (-aminoacids), $0.07 \%$ CSM (-relevant

$\mathrm{SD} / \mathrm{SG} / \mathrm{SGal}$ metabolite) with $2 \%$ glucose $/ 3 \%$ glycerol $/ 2 \%$ lactate

YPD/YPG/YPGal/YPLac medium

$1 \%$ yeast extract, $2 \%$ peptone, $2 \%$ glucose $/ 3 \%$ glycerol/

Zymolyase buffer

$2 \%$ galactose $/ 2 \%$ lactate ( $\mathrm{pH} 6.0$ with $\mathrm{KOH}$ for YPLac)

$20 \mathrm{mM} \mathrm{KPO}_{4} \mathrm{pH} 7.4,1.2 \mathrm{M}$ sorbitol, $0.57 \mathrm{~g} / 1$ zymolyase

\subsection{Methods}

\subsubsection{Working with Microorganisms}

\subsubsection{Growth conditions for E. coli}

Using standard procedures, E. coli XL1 Blue was grown at $37^{\circ} \mathrm{C}$ in lysogeny broth (LB) (Green et al., 2012). For selection, $0.1 \mathrm{~g} / 1$ ampicillin was added. Same applied for using plates, supplemented with $15 \mathrm{~g} / \mathrm{l}$ agar. Plasmid carrying E. coli were preserved as cryo stocks at $-80{ }^{\circ} \mathrm{C}$ by adding $800 \mu \mathrm{l}$ of liquid culture to $200 \mu \mathrm{l}$ sterile $80 \%$ glycerol.

\subsubsection{General handling and growth conditions for yeast}

The standard culturing of all yeast strains was obtained in YP medium (Table 2-9), supplemented with glucose (YPD), glycerol (YPG), galactose (YPGal) or lactate (YPLac; $\mathrm{pH} 6.0$ with $\mathrm{KOH}$ ), at a growth temperature of $30{ }^{\circ} \mathrm{C}$ and shaking at $160-220 \mathrm{rpm}$, unless it is mentioned otherwise. 
For selection of antibiotic resistant strains (KANMX4, NATNT2), 200 mg/l G418 sulphate or $100 \mathrm{mg} / 1$ nourseothricin, respectively, were added. Selective minimal media were used for selection for auxotrophic marker (HISMX6) or culturing of plasmid-carrying strains (URA), containing yeast nitrogen base without aminoacids(YNB), complete supplement mixture (CSM) lacking the relevant metabolite and glucose (SD), glycerol (SG), galactose (SGal) (Table 2-9). $25 \mathrm{~g} / \mathrm{l}$ agar was added for casting plates.

For strain preservation as cryo stocks, $800 \mu \mathrm{l}$ of a liquid overnight culture in YPD or SD were added to $200 \mu \mathrm{l}$ sterile $80 \%$ glycerol and stored at $-80{ }^{\circ} \mathrm{C}$.

In general, the maintenance of the genome is a high priority and the strains were freshly streaked from previously prepared cryo stocks for analysis. The strains were kept on plates for 1-3 weeks and restreaked if needed.

\subsubsection{Growth test}

For comparing the yeast growth in different media, the corresponding strain's cells were cultured overnight in YPD or the appropriate selective minimal medium SD, diluted to an optic density (at $600 \mathrm{~nm}$; $\mathrm{OD}_{600}$ ) of 0.5 and grown for $2 \mathrm{~h}$. After thorough washing in sterile water, the cells were spotted in serial dilution $\left(\mathrm{OD}_{600}\right.$ 1-0.0001) on plates. As a fermentable carbon source, glucose and as non-fermentable carbon sources, glycerol or lactate were used. Plates were incubated at $30^{\circ} \mathrm{C}, 25^{\circ} \mathrm{C}$ and/or $37^{\circ} \mathrm{C}$ for $2-5$ days.

\subsubsection{Whole cell lysate preparation of yeast}

For analyzing the protein content of whole cells an appropriate amount of cell material was harvested either from plate or an equivalent amount from liquid culture ( $\mathrm{OD}_{600}$ 1.0). Cells were resuspended in cold $25 \%$ trichloroacetic acid (TCA) (diluted with phosphate buffered saline, PBS) and incubated at $-80{ }^{\circ} \mathrm{C}$ for $30 \mathrm{~min}$ for protein precipitation. After a washing with $80 \%$ ice-cold acetone, pellets were dried at room temperature, finally resuspended in $0.1 \mathrm{M} \mathrm{NaOH}$ and $1 \%$ sodium dodecyl sulfate (SDS) containing solution and incubated at $30{ }^{\circ} \mathrm{C}$ for at least $15 \mathrm{~min}$ in a shaking motion. SDS-sample buffer was added and samples were subjected to SDSPAGE analysis, as described in section 2.2.3.2.

\subsubsection{Isolation of mitochondria}

Essentially, isolation followed previously described procedures (Meisinger, Pfanner and Truscott, 2006). Yeast was grown in YPG or SG (YPGal or SGal for respiratory defective strains) in at least two separate precultures prior to a 21 mainculture, raised until an $\mathrm{OD}_{600}$ of 1.0-4.0. Cells were harvested for $15 \mathrm{~min}$ at $4000 \mathrm{rpm}$ (Sorvall) and washed in water once (Beckmann-Coulter). Then, pellets were incubated for $30 \mathrm{~min}$ at $30^{\circ} \mathrm{C}$ in $2 \mathrm{ml} / \mathrm{g}$ cell wet weight 
(CWW) dithiotreitol (DT'T) buffer (Table 2-9) and after a centrifugation of $4000 \mathrm{rpm}, 8 \mathrm{~min}$, washed with 1.2 M sorbitol. In order to degrade the cell wall, cells were resuspended in $7 \mathrm{ml} / \mathrm{g}_{\mathrm{CWw}}$ zymolyase buffer (Table 2-9) and incubated at $30{ }^{\circ} \mathrm{C}, 90 \mathrm{rpm}$ for $1-2 \mathrm{~h}$ with allowing $\mathrm{O}_{2}$ supply. The resulting spheroblasts were spun down at $3000 \mathrm{rpm}$ for $8 \mathrm{~min}$, washed again in $100 \mathrm{ml}$ cold zymolyase buffer without enzyme and resuspended in $7 \mathrm{ml} / \mathrm{g}_{\mathrm{cww}}$ cold homogenization buffer (Table 2-9). Cells were opened with a cell homogenizer (potter) at $900 \mathrm{rpm}$ for 15 strokes on ice. The homogenate was first centrifuged at $3000 \mathrm{rpm}$ for $5 \mathrm{~min}$, $4{ }^{\circ} \mathrm{C}$ and the supernatant was subjected to an additional step of $4000 \mathrm{rpm}$ for $10 \mathrm{~min}, 4^{\circ} \mathrm{C}$. Crude mitochondria fraction was obtained by centrifugation at $12000 \mathrm{rpm}$ for $15 \mathrm{~min}, 4^{\circ} \mathrm{C}$ and washed with SEM buffer (Table 2-9) with $1 \mathrm{mM} \mathrm{PMSF.} \mathrm{Finally,} \mathrm{mitochondria} \mathrm{were}$ resuspended in SEM buffer, adjusted to a protein concentration $10 \mathrm{mg} / \mathrm{ml}$ using Bradford assay (see 2.2.3.1), frozen in liquid nitrogen and stored at $-80{ }^{\circ} \mathrm{C}$.

\subsubsection{Molecular biology methods}

\subsubsection{Transformation of $E$. coli}

Competent E. coli cells ( $50 \mu \mathrm{l}$ aliquot) were thawed on ice, mixed with $5 \mu \mathrm{l}$ of ligation product or 100-200 ng plasmid DNA, incubated for $30 \mathrm{~min}$ on ice and underwent a heat shock at $42{ }^{\circ} \mathrm{C}$ for $1.5 \mathrm{~min}$. After chilling down on ice again for $5 \mathrm{~min}$, cells were resuspended in LB, incubated at $37^{\circ} \mathrm{C}, 1000 \mathrm{rpm}$ for at least $1 \mathrm{~h}$ and finally plated on LB plates containing ampicillin (see 2.2.1.1). Plates were incubated at $37^{\circ} \mathrm{C}$ overnight. Single colonies were picked and transferred into liquid culture for further processing after section 2.2.2.2.

\subsubsection{Plasmid DNA isolation}

For purifying plasmids expressed in E. coli, $2 \mathrm{ml}$ culture (see 2.2.1.1) was harvested and the Wizard $^{\circledR}$ Plus SV Minipreps DNA Purification System (Promega) was used, following the manufacturer's protocol. The isolated plasmid DNA was finally eluted with nuclease free water, concentration measured with the Nanodrop (Thermo Scientific) and stored at $-20^{\circ} \mathrm{C}$.

\subsubsection{Transformation of yeast}

Yeast transformation followed essentially the procedure after Knop et al. 1999 (Knop et al., 1999). For preparing competent yeast cells, overnight culture, grown in YPD, was diluted to $\mathrm{OD}_{600}=0.25$, incubated again for $\sim 3 \mathrm{~h}$ at $30^{\circ} \mathrm{C}$ (until $\mathrm{OD}_{600}=0.5-0.7$ ) and harvested at $2000 \mathrm{rpm}, 5 \mathrm{~min}$. Pellet was washed with sterile water and SORB buffer (Table 2-9). After removing SORB buffer, cells were resuspended in $360 \mu$ SORB buffer per $50 \mathrm{ml}$ culture and $40 \mu \mathrm{l}$ herring sperm carrier DNA (previously heated at $95^{\circ} \mathrm{C}, 10 \mathrm{~min}$, put on ice). Competent cells were aliquoted into $50 \mu \mathrm{l}$ and were either stored at $-80{ }^{\circ} \mathrm{C}$, without shock freezing, or 
subsequently used for transformation. Depending on the desired transformation, 1-5 $\mu$ l of plasmid DNA or $10 \mu \mathrm{l}$ of PCR product were added to the competent cell mixture followed by the 6-fold volume $(300 \mu \mathrm{l}$ ) of PEG buffer (Table 2-9), incubated for $30 \mathrm{~min}$ at room temperature, until DMSO was added to an end concentration of $10 \%$ and heat shock was performed at $42{ }^{\circ} \mathrm{C}, 15 \mathrm{~min}$. After centrifugation (4000 rpm, $5 \mathrm{~min}$ ), cells were resuspended in fresh YPD and either spread directly on selective plates in case of auxotrophic marker genes, or incubated for another $2 \mathrm{~h}$ at $30^{\circ} \mathrm{C}$ in case of antibiotic resistance genes before spreading. Colonies were picked after 3-7 days, and streaked again on selective plates before single colonies were tested for expression and used for further experiments.

\subsubsection{Yeast genomic DNA isolation}

Yeast genomic DNA is isolated from a $20 \mathrm{ml}$ overnight culture, harvested at $2000 \mathrm{rpm}, 10 \mathrm{~min}$. Cells are resuspended in $1 \mathrm{ml}$ DT'T buffer (Table 2-9), incubated at $30{ }^{\circ} \mathrm{C}, 30 \mathrm{~min}, 750 \mathrm{rpm}$ and washed with $1 \mathrm{ml}$ zymolyase buffer (Table 2-9) without enzyme before incubation with $1 \mathrm{ml}$ of the same buffer, enzyme added, at $30^{\circ} \mathrm{C}, 1 \mathrm{~h}, 750 \mathrm{rpm}$, allowing $\mathrm{O}_{2}$ supply. The spheroblasts are spun down with $4000 \mathrm{rpm}, 10 \mathrm{~min}$ and DNA is solubilized with $0.1 \mathrm{M} \mathrm{NaOH}$, subsequently diluted to $33 \mathrm{mM} \mathrm{NaOH}$. An incubation at $100{ }^{\circ} \mathrm{C}$ for 5-10 min followed and samples were chilled down on ice. Precipitates were spun down at $13000 \mathrm{rpm}, 10 \mathrm{~min}, 4^{\circ} \mathrm{C}$, the supernatant was transferred and diluted with water $1|100-1| 1000$, concentration was measured using the Nanodrop (Thermo Scientific), and stored at $-20^{\circ} \mathrm{C}$.

In cases of simple confirmation analysis, small amounts of yeast cell material were scraped from plate and microwaved for $1 \mathrm{~min}$ before subjected to polymerase chain reaction, as described below in 2.2.2.5.

\subsubsection{PCR}

Polymerase chain reaction (PCR) was performed for amplifying DNA segments from plasmids, genomic DNA or previous PCR products by using KOD polymerase (Novagen) following the manufacturer's protocol. Recommended cycling conditions were adjusted for improving the yield. Polymerase activation was achieved by incubation at $95^{\circ} \mathrm{C}, 5 \mathrm{~min}$ and depending on the appropriate primer pair (listed in Table 2-4), the first 10 cycles were performed as it follows: Denaturation at $95^{\circ} \mathrm{C}, 20 \mathrm{~s}$, annealing at $52-58{ }^{\circ} \mathrm{C}, 10 \mathrm{~s}$ and extension at $70{ }^{\circ} \mathrm{C}, 15-25 \mathrm{~s} / \mathrm{kb}$ depending on target size. The following 25 cycles ran with a reduced annealing temperature of $46-52{ }^{\circ} \mathrm{C}, 10 \mathrm{~s}$, and extension was completed with $70{ }^{\circ} \mathrm{C}, 2 \mathrm{~min}$. Analysis and separation of the PCR fragments was accomplished, after adding 1x loading dye (Thermo Scientific), by horizontal agarose gel electrophoresis $\left(1.5 \%\right.$ agarose, $1 \mathrm{x}$ ROTI ${ }^{\circledR}$ GelStain in TAE buffer; BioRad chamber) at $120 \mathrm{~V}$ for $20-30 \mathrm{~min}$. With the help of GeneRuler DNA ladder mix 
(Thermo Scientific) as a standard, PCR fragments were visualized on a UV-table and purified from gel with Wizard ${ }^{\circledR}$ SV Gel and PCR Clean-Up System (Promega), according to manufacturer's instructions. Concentration was measured with the Nanodrop (Thermo Scientific) and PCR samples were stored at $-20^{\circ} \mathrm{C}$ until further use.

\subsubsection{Cloning of plasmids}

Generating plasmids for the purposes of this study (Table 2-5) followed principles of standard protocols (Green et al., 2012). Therefore, purified PCR sample (2.2.2.5) and the desired plasmid were used for restriction digestion with adequate FastDigest enzymes (Thermo Scientific) following the manufacturer's protocol. The incubation proceeded at $37^{\circ} \mathrm{C}$ for $30 \mathrm{~min}$, digested PCR product (insert) and plasmid (backbone) were cleaned up, following the same protocol as for PCR products (2.2.2.5), and used for ligation with Rapid DNA Ligation Kit (Thermo Scientific). Pursuing the manufacturer's user guide, 10-100 ng of vector and an applicable amount of insert were utilized for ligation. Insert concentration was calculated with the following formula: mass $_{\text {insert }}(\mathrm{ng})=3 \cdot$ mass $_{\text {vector }}(\mathrm{ng}) \cdot$ length $_{\text {insert }}(\mathrm{bp}) /$ length $_{\text {vector }}(\mathrm{bp})$ to gain an insert to vector molar ratio of $3 \mid 1$. Ligation product was used for transformation of E. coli as described in 2.2.2.1. Plasmid DNA was purified as in section 2.2.2.2 and successful cloning was verified by restriction digestion analysis and further by sequencing (Microsynth SEQLAB, Göttingen).

\subsubsection{Chromosomal deletions/insertions in yeast}

For deleting genes or integration of tags genomically in yeast, a PCR based strategy was used following standard procedures (Longtine et al., 1998; Knop et al., 1999; Janke et al., 2004). In case of deletions HIS3MX6, KANMX4 (Longtine et al., 1998) or NATNT2 (Janke et al., 2004) cassettes were amplified using primers with homology region sequences up- and downstream ( $500 \mathrm{bp}$ ) of the targeted open reading frame (ORF). For insertion of 3HA-tag, plasmid pYM2 (Knop et al., 1999) was utilized and primers contained homology region sequences matching the 3'-end of the ORF excluding the Stop codon and 250 bp downstream the ORF, respectively (listed in Table 2-4). PCR products were used for transformation as indicated in section 2.2.2.3. True-positive clones were confirmed with PCR and/or western blotting

\subsubsection{In vitro transcription and translation}

For labeling precursor proteins with $\left[{ }^{35} \mathrm{~S}\right]$-methionine, mMassage mMachine SP6 kit (Invitrogen) was first used to produce capped mRNA. PCR products containing SP6 promoter in front of the ORF were generated from either plasmid or yeast genomic DNA, additionally considering the number of encoded methionine. According to the manufacturer's protocol, for a $20 \mu \mathrm{l}$ reaction, $1 \mathrm{x}$ NTP/CAP, $1 \mathrm{x}$ reaction buffer, $1 \mu \mathrm{g}$ PCR product and $2 \mu \mathrm{l}$ enzyme mix were mixed 
and incubated at $37^{\circ} \mathrm{C}$ for $90 \mathrm{~min} .1 \mu \mathrm{l}$ Turbo DNase was added to remove DNA template and incubation at $37^{\circ} \mathrm{C}$ for 15 min followed. RNA was precipitated by adding $30 \mu \mathrm{LiCl}$ solution, optionally adding $30 \mu \mathrm{l}$ nuclease-free water and incubating at $-20{ }^{\circ} \mathrm{C}$ for at least $30 \mathrm{~min}$. RNA was pelleted at $14000 \mathrm{rpm}, 15 \mathrm{~min}, 4^{\circ} \mathrm{C}$, washed with cold $70 \%$ ethanol, dried, resuspended in $50 \mu \mathrm{l}$ nuclease-free water, concentration was measured at the Nanodrop (Thermo Scientific)and stored at $-80^{\circ} \mathrm{C}$. The translation reaction was then carried out with the Flexi ${ }^{\circledR}$ Reticulocyte Lysate System (Promega). In order to enhance efficiency, RNA was heated prior usage for $3 \mathrm{~min}$ at $65^{\circ} \mathrm{C}$ and $1 \mu \mathrm{g}$ RNA was used per $50 \mu \mathrm{l}$ reaction. For this, $33 \mu \mathrm{l} \mathrm{Flexi}{ }^{\circledR}$ Reticulocyte Lysate, $1 \mu \mathrm{l}$ amino acid mix without methionine $(1 \mathrm{mM}), 70-120 \mathrm{mM} \mathrm{KCl}, 0-2 \mathrm{mM}$ $\mathrm{MgAc}_{2}, 0-2 \mathrm{mM}$ DTT, depending on the protein synthesized (see Table 2-10) and $30 \mu \mathrm{Ci}$ $\left[{ }^{35} \mathrm{~S}\right]$-Met were mixed and incubated for $90 \mathrm{~min}$ at $30{ }^{\circ} \mathrm{C}$. When adequate plasmids were available, transcription and translation were carried out in a coupled reaction from plasmid DNA, containing SP6-promoter, with using TNT ${ }^{\circledR}$ Quick Coupled Transcription Translation kit (Promega). Per $50 \mu$ reaction, $40 \mu$ l of $\mathrm{TNT}^{\circledR}$ Quick Master Mix and $20 \mu \mathrm{Ci}\left[{ }^{35} \mathrm{~S}\right]$-Met were added to $1 \mu \mathrm{g}$ plasmid DNA. Lysates were shock-frozen with liquid nitrogen and stored at $-80{ }^{\circ} \mathrm{C}$.

In case of $\left[{ }^{35} \mathrm{~S}\right]$-labeled Cox12, lysate was precipitated for reducing and unfolding the protein prior to import reactions. Thus, prepared $\left[{ }^{35} \mathrm{~S}\right]$-Cox12 lysate, $20 \mathrm{mM}$ EDTA (pH 8.0 with $\mathrm{NaOH}$ ), sat. $\left(\mathrm{NH}_{4}\right)_{2} \mathrm{SO}_{4}$ (in total $v \mid v$ ratio is $2 \mid 1$, added in two steps) were mixed thoroughly before incubating for $30 \mathrm{~min}$ on ice and spinning at $14000 \mathrm{rpm}, 15 \mathrm{~min}, 4^{\circ} \mathrm{C}$. The pellet is resuspended in $1 / 3$ of the initial volume with urea buffer incubated at room temperature at $450 \mathrm{rpm}$ for $15 \mathrm{~min}$, spun down again at $14000 \mathrm{rpm}, 15 \mathrm{~min}, 4^{\circ} \mathrm{C}$. Supernatant was used as lysate.

Table 2-10: Conditions for Flexi ${ }^{\circledR}$ Reticulocyte Lysate System.

\begin{tabular}{lll} 
Precursor & KCl $[\mathbf{m M}]$ & $\mathbf{M g A c}_{2}[\mathbf{m M}]$ \\
\hline \hline Cox12 & 70 & 0.95 \\
Cox13 & 70 & 0.95
\end{tabular}

\subsubsection{Protein biochemistry methods}

\subsubsection{Bradford assay - determination of protein concentration}

Protein Concentration was determined by using Roti ${ }^{\circledR}$-Quant (Roth) after Bradford (Bradford, 1976). For this, a standard curve $0-75 \mu \mathrm{g} / \mu \mathrm{l}$ of bovine immunoglobuline was detected, and three 
different dilutions per sample were measured, using Roti ${ }^{\circledR}$-Quant in a $1 \mid 10$ dilution. Measurement was accomplished using the UV-Spectrophotometer (Varian) at $595 \mathrm{~nm}$.

\subsubsection{SDS-PAGE}

For separating denatured proteins, SDS polyacrylamide gel electrophoresis (SDS-PAGE) was carried out based on principles of Laemmli (Laemmli, 1970). Depending on the size range of the examined proteins, uniform resolving gels were casted in different polyacrylamide concentrations ranging from 16-10\%, using a commercial 30\%/0.8\% acrylamide/bisacrylamide solution (Roth) mixed along with 386 mM TRIS pH 8.8 with HCl, $0.1 \%$ SDS. To gain a better resolution in some cases, SDS gradient gels from 10-16\% polyacrylamide were applied. The same protocol for preparing the acrylamide mixtures was used, except for adding $100 \mathrm{mM}$ sucrose and $3.5 \%$ glycerol to the heavy gel mix. For casting the gradient, a custommade gradient mixer was used. A stacking gel with $5 \%$ polyacrylamide, $80 \mathrm{mM}$ TRIS pH 6.8 with $\mathrm{HCl}, 0.1 \%$ SDS was poured over the resolving gel. Ammonium persulfate (APS) and tetramethylenediamine (TEMED) were used for polymerization. Electrophoresis was performed in a custom-made midi system or the Mini-Protean II (BioRad) with $30 \mathrm{~mA} /$ gel with SDS running buffer (Table 2-9).

Urea SDS-PAGE helped to resolve proteins of low molecular weight. For this, the resolving gel consisted of $17.5 \%$ acrylamide (60\%/0.8\% acrylamide/bis-acrylamide solution), $683 \mathrm{mM}$ TRIS pH 8.8 with $\mathrm{HCl}, 7.8 \mathrm{mM} \mathrm{NaCl}, 5.4 \mathrm{M}$ urea and $0.1 \%$ SDS. The stacking gel was casted with $5.4 \%$ acrylamide (60\%/0.8 \%), 108 mM TRIS pH 6.8 with HCl, 3.3 M urea, $0.12 \%$ SDS. APS and TEMED were used for polymerization. Electrophoresis was carried out under same conditions as standard SDS-PAGE with urea SDS running buffer (Table 2-9).

Tricine SDS-PAGE was used in order to aim for the best resolution for radioactively labeled translation products, as described in section 2.2.5.2. Similar to Schägger (Schägger, 2006), a gradient of 10-18 \% polyacrylamide was applied, using a $48 \% / 1.5 \%$ acrylamide/bis-acrylamide solution along with $1 \mathrm{M}$ TRIS pH 8.45 with HCl, $0.1 \%$ SDS and $13 \%$ glycerol in the heavy gel mix, in a custom-made gradient mixer, and overlaid with stacking gel (4\% polyacrylamide, $1 \mathrm{M}$ TRIS pH 8.45 with $\mathrm{HCl}, 0.1 \%$ SDS). APS and TEMED were used for polymerization. For electrophoresis, a custom-made midi system and $25 \mathrm{~mA} /$ gel with Tricine SDS cathode buffer and Tricine SDS anode buffer were used (Table 2-9).

Before loading onto SDS-PAGE, SDS sample buffer (Table 2-9) was added and samples were incubated at $95^{\circ} \mathrm{C}$ for $5 \mathrm{~min}$ or under mild agitation at $37^{\circ} \mathrm{C}$ for $20 \mathrm{~min}$. If necessary, samples were stored at $-20{ }^{\circ} \mathrm{C}$. 


\subsubsection{Blue-Native PAGE}

Native separation of protein complexes was accomplished by blue-native PAGE (BN-PAGE), following previously standardized principles of Dekker and colleagues (Schägger and von Jagow, 1991; Dekker et al., 1996; Wittig, Braun and Schägger, 2006). Separation gels with a polyacrylamide gradient of 4-10\%, 4-13\%, 4-16\%, 6-10\% were applied, using a 48 \%/1.5\% acrylamide/bis-acrylamide solution along with $50 \mathrm{mM}$ BIS-TRIS $\mathrm{pH} 7.0$ with $\mathrm{HCl}$, $66.67 \mathrm{mM}$ 6-aminocaproic acid and $20 \%$ glycerol for the heavy gel mix. A custom-made gradient mixer helped with casting consistent gradient gels. A $4 \%$ polyacrylamide gel with $50 \mathrm{mM}$ BIS-TRIS pH 7.0 with $\mathrm{HCl}, 66.67 \mathrm{mM}$ 6-aminocaproic acid served as stacking gel. APS and TEMED were used for polymerization. Gels were cast and ran in the SE600 Ruby system (Hoefer, GE Healthcare) with BN-cathode buffer (Table 2-9), first containing $0.02 \%$ Coomassie Brilliant Blue G250, which was replaced with clear cathode buffer after 1/3 of the run, and BN-anode (Table 2-9) buffer at $15 \mathrm{~mA} / \mathrm{gel}$. Running buffers were precooled to $4{ }^{\circ} \mathrm{C}$, as well as loading samples was completed at $4{ }^{\circ} \mathrm{C}$.

For sample preparation, mitochondria were solubilized in either $1 \%$ digitonin or $0.6 \% \mathrm{DDM}$ BN solubilization buffer (Table 2-9) to a concentration of $1 \mu \mathrm{g} / \mu \mathrm{l}$ for $20 \mathrm{~min}$ on ice. Insoluble material was spun down $\left(14000 \mathrm{rpm}, 15 \mathrm{~min}, 4^{\circ} \mathrm{C}\right)$ and $\mathrm{BN}$ sample buffer was added prior to loading.

In order to separate complexes in $2^{\text {nd }}$ dimension, whole BN lanes were incubated for $30 \mathrm{~min}$ in $50 \mathrm{mM}$ DTT containing SDS running buffer (Table 2-9) and subsequently cast into a SDS gel following the mentioned principles in section 2.2.3.2.

\subsubsection{Western blotting and immunodetection}

In order to detect proteins via immunodetection, standard procedures as described by Gallagher and colleagues were used (Gallagher et al., 2004). First, the semi-dry blot system of PEQLAB was applied to transfer proteins from polyacrylamide gels onto PVDF membranes (Merck Millipore). After short activation in methanol, the membrane was assembled together with the gel and filter paper (Heinemann Labortechnik), soaked prior in transfer buffer (Table 2-9). Protein transfer was completed after $2.5 \mathrm{~h}$ with $25 \mathrm{~V}, 250 \mathrm{~mA}$ in case of SDS-PAGE and after $3 \mathrm{~h}$ with $25 \mathrm{~V}, 400 \mathrm{~mA}$ in case of BN-PAGE. To visualize proteins and protein molecular weight marker, membranes were stained and destained as described in 2.2.3.5. Then, the membrane was cut into stripes, according to the respective proteins to be detected, destained and reactivated in methanol. TBS-T (Table 2-9) supplemented with $5 \%$ milk was used for unspecific blocking for $1 \mathrm{~h}$ at room temperature or overnight at $4{ }^{\circ} \mathrm{C}$ under mild agitation. Specific home-made polyclonal antibodies, diluted in TBS-T with $5 \%$ milk, or commercial 
antibodies, diluted in TBS-T, were applied for immunodecoration and incubated for $1 \mathrm{~h}$ at room temperature or overnight at $4{ }^{\circ} \mathrm{C}$ under mild agitation. After three washing steps with TBS-T for $10 \mathrm{~min}$, membranes were incubated with adequate secondary antibodies (1 | 5 000-1| 10000 dilution) for $1 \mathrm{~h}$ at room temperature. The same washing procedure was used, and protein signals were visualized using enhanced chemiluminescence (ECL) Pierce ${ }^{\circledR}$ ECL Western Blotting Detection Reagent (Thermo Scientific) on either X-ray films (Fujifilm) or the Amersham $^{\mathrm{TM}}$ ImageQuant ${ }^{\mathrm{TM}} 800$ system (GE Healthcare).

\subsubsection{Coomassie staining of membranes and gels}

In order to visualize proteins in polyacrylamide gels or on membranes after PAGE and western blot, they were stained with Coomassie Brilliant Blue Solution (Table 2-9) and destained with $10 \%$ HAc, $40 \%$ ethanol solution until bands were apparent.

\subsubsection{Autoradiography}

For detecting radioactively labeled proteins, gels underwent either western blotting followed by Coomassie staining as described in sections 2.2.3.4 and 2.2.3.5, or gels were directly stained and destained as mentioned in 2.2.3.5 and subsequently dried, on top of two filter paper and covered by plastic foil, on a geldryer (LTF Labortechnik) at $65^{\circ} \mathrm{C}$ for $2-4 \mathrm{~h}$. Protein size standard was subsequently marked with radioactive ink, covered with sticking tape and exposed on storage phosphor screens (GE Healthcare), both the dried membrane and the dried gel. Signals were digitalized using the Amersham ${ }^{\mathrm{TM}}$ Typhoon $^{\mathrm{TM}}$ Scanner (GE Healthcare) and quantified using Fiji/ImageJ (Schindelin et al., 2012).

\subsubsection{Steady state analysis of protein levels}

To probe and compare steady state levels of mitochondrial proteins, isolated mitochondria were directly employed for SDS-PAGE. Therefore, mitochondria were resuspended in SDS-sample buffer as in 2.2.3.2 to $1 \mu \mathrm{g} / \mu \mathrm{l}$ and incubated at $95^{\circ} \mathrm{C}$ for $5 \mathrm{~min}$.

\subsubsection{Protease protection assay}

Submitochondrial localization of proteins was obtained by protease treatment as described previously (Vukotic et al., 2012). For this, intact mitochondria were incubated with either hypotonic EM buffer (Table 2-9) to be converted to mitoplasts or with isotonic SEM buffer (Table 2-9) to be preserved as such. As a control, mitochondria were lysed with $1 \%$ Triton

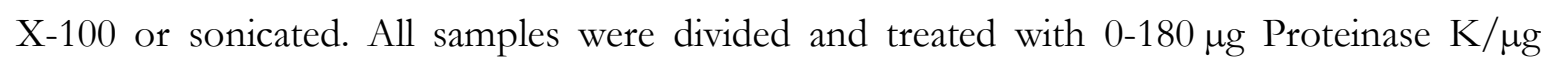
mitochondria. Proteinase $\mathrm{K}$ was inactivated with $2 \mathrm{mM}$ PMSF and samples were applied to SDS-PAGE as in 2.2.3.2. 


\subsubsection{Purification of protein complexes}

\subsubsection{Crosslinking of Antibodies to PA-Sepharose beads}

Prior to co-immunoprecipitation, specific antibodies were bound to PA-Sepharose beads via crosslinking, following previously used protocols (Bareth et al., 2013). Sera were applied in 1|8 dilutions in $0.1 \mathrm{M} \mathrm{KPO}_{4}$ buffer ( $\mathrm{pH} 7.4$ ) onto $25 \mu \mathrm{l}$ PA-Sepharose beads, using mobicol columns (mobitec), and incubated for $1 \mathrm{~h}$ at room temperature under permanent inversion. Then, beads were washed with $0.1 \mathrm{M}$ sodium borate $(\mathrm{pH} 9.0$ with $\mathrm{NaOH}$ ) and afterwards crosslinked with $5 \mathrm{mg} / \mathrm{ml}$ dimethyl pimelidate (DMP) in $0.1 \mathrm{M}$ sodium borate (pH 9.0 with $\mathrm{NaOH}$ ) for $30 \mathrm{~min}$ at room temperature under permanent inversion. Crosslinking reaction was quenched with $1 \mathrm{M}$ TRIS ( $\mathrm{pH} 7.4$ with $\mathrm{HCl}$ ) for $2 \mathrm{~h}$ at room temperature or overnight at $4{ }^{\circ} \mathrm{C}$ under permanent inversion. At the end, beads were washed with TBS or PBS for at least three times and stored in TBS or PBS supplemented with $2 \mathrm{mMNaN}_{3}$ at $4{ }^{\circ} \mathrm{C}$. Before and after usage, beads were washed twice with $\mathrm{NH}_{4}$ Ac buffer ( $\mathrm{pH}$ 3.4) followed by two TBS or PBS washes.

\subsubsection{Co-immunoprecipitation}

Isolation of protein complexes by co-immunoprecipitation (IP) was essentially performed as described before (Bareth et al., 2013). Specific polyclonal antibodies against Rcf1, Rcf2, Rcf3, Cox2, Qcr8, Cox12, Cox13 were applied as indicated in each experiment. Mitochondria were solubilized in IP solubilization buffer (Table 2-9) containing either $1 \%$ digitonin, $0.6 \%$ DDM or $0.5 \%$ Triton X-100 with $0.1 \%$ SDS to $1 \mu \mathrm{g} / \mu \mathrm{l}$ for $40 \mathrm{~min}$ at $4{ }^{\circ} \mathrm{C}$ under mild agitation, and lysate was cleared at $14000 \mathrm{rpm}, 15 \mathrm{~min}, 4^{\circ} \mathrm{C}$. A total sample was taken and the supernatant was split and applied onto appropriate antibody-coupled columns, incubating for $1 \mathrm{~h}$ at $4{ }^{\circ} \mathrm{C}$ under permanent inversion, commercial control columns (Thermo Scientific) served as a control for specific co-IP. After intensive washing with the same buffer as mentioned above but without detergent in case of DDM, $0.3 \%$ digitonin or $0.3 \%$ Triton X-100, precipitated protein complexes could be eluted with $0.1 \mathrm{M}$ glycine $(\mathrm{pH} 2.8$ with $\mathrm{HCl}$ ) in a two-step elution. To this end, glycine was added, columns were incubated at $16{ }^{\circ} \mathrm{C}$ for $4 \mathrm{~min}, \operatorname{spun}(200 \mathrm{xg}, 1 \mathrm{~min}$, $4{ }^{\circ} \mathrm{C}$ ), repeating it again. Small amounts of 1 M TRIS ( $\mathrm{pH}$ 11.5) were used for neutralization and samples were subjected to SDS-PAGE as described in 2.2.3.2.

\subsubsection{FLAG isolation}

For native isolation of protein complexes by FLAG isolation, FLAG 2-M Affinity gel (Sigma-Aldrich) with $20 \mu \mathrm{l}$ beads per $1 \mathrm{mg}$ mitochondria were used. Solubilization conditions and handling of columns was following same principles as in section 2.2.4.2. Elution was completed natively with 1x FLAG peptide (Sigma-Aldrich) in wash buffer in a two-step 
application, each time incubating for $5 \mathrm{~min}$ at room temperature. Samples were either applied for SDS-PAGE or BN-PAGE.

\subsubsection{HA isolation}

In order to isolate protein complexes via HA-tag, $20 \mu$ Pierce ${ }^{\mathrm{TM}}$ Anti-HA Agarose (Thermo Scientific) beads per $500 \mu \mathrm{g}$ mitochondria were applied. HA-isolation followed the exact same protocol as described for co-IP in 2.2.4.2. The elution followed also the non-native protocol with $0.1 \mathrm{M}$ glycine ( $\mathrm{pH} 2.8$ with $\mathrm{HCl}$ ) mentioned in 2.2.4.2, although native elution with HA-peptide (Thermo Fisher) would have been possible.

\subsubsection{Specialized assays}

\subsubsection{In vitro protein import and assembly}

First, proteins were radioactively labeled as described in section 2.2.2.8. In vitro import and assembly of the $\left[{ }^{35} \mathrm{~S}\right]$-Met labeled proteins into isolated mitochondria was carried out similar to Ryan et al. (Ryan, Voos and Pfanner, 2001). For this, mitochondria were resuspended in import buffer (Table 2-9) supplemented with $2 \mathrm{mM} \mathrm{NADH}$ and $2 \mathrm{mM} \mathrm{ATP}$ (pH 7.0 with $\mathrm{HCl}$ ) and in case for assembly reactions additionally with $0.1 \mathrm{mg} / \mathrm{ml}$ creatin kinase and $5 \mathrm{mM}$ creatine phosphate for ATP regeneration. In case of $\left[{ }^{35} \mathrm{~S}\right]-\operatorname{Cox} 12$, import buffer without BSA was used. As a negative control, one import reaction was mixed with $8 \mu \mathrm{M}$ antimycin, $1 \mu \mathrm{M}$ valinomycin and $20 \mu \mathrm{M}$ oligomycin (AVO) to dissipate membrane potential and samples were pre-incubated for $3 \mathrm{~min}$ at $450 \mathrm{rpm}, 25^{\circ} \mathrm{C}$. Import was started by adding $-\left[{ }^{35} \mathrm{~S}\right]$-labeled precursor proteins and stopped after various time-points by adding AVO-mix. Then, samples were treated either with Proteinase K $(25 \mu \mathrm{g} / \mathrm{ml})$, to remove unimported protein, or instantly pelleted (14 $000 \mathrm{rpm}$, $15 \mathrm{~min}, 4^{\circ} \mathrm{C}$ ) and washed with cold SEM buffer. For SDS-analysis of imported protein, samples were applied to SDS-PAGE as in section 2.2.3.2. In order to analyze the assembly of radioactively labeled protein in protein complexes, samples were solubilized and applied to BNPAGE as described in section 2.2.3.3.

In the end, membranes or gels were handled as described in section 2.2.3.5 and samples were analyzed with autoradiography as mentioned in section 2.2.3.6.

\subsubsection{In vitro translation assay in isolated mitochondria}

Isolated mitochondria were subjected into translation buffer and mitochondrial translation products were radiolabeled for $10 \mathrm{~min}$ with $20 \mu \mathrm{M}\left[{ }^{35} \mathrm{~S}\right]$ methionine at $30{ }^{\circ} \mathrm{C}$, in principle as described previously (Westermann, Herrmann and Neupert, 2001). The reaction was stopped by adding $40 \mathrm{mM}$ cold methionine. Mitochondria were reisolated and washed in SEM buffer (Table 2-9). Samples were either used for further co-immunoprecipitation as in 2.2.4.2 or 
directly analyzed with Tricine SDS-PAGE according to 2.2.3.2. Proteins were transferred to PVDF membranes as described in section 2.2.3.4 and labeling was analyzed via autoradiography (2.2.3.6). Afterwards, an additional probing with antibodies (2.2.3.4) completed the analysis.

\subsubsection{Crosslinking with chemical crosslinker}

A second crosslinking reagent used in this study was bis(sulfosuccinimidyl)suberate $\left(\mathrm{BS}^{3}\right.$; Thermo Scientific). As a homobifunctional crosslinker it is reacting with primary amino groups at $\mathrm{pH}$ 7-9. To this end, isolated mitochondria were resuspended in crosslinking buffer Table $2-9, \mathrm{BS}^{3}$ was added to an end concentration of $5 \mathrm{mM}$ and incubated at room temperature for $1 \mathrm{~h}$. Reaction was quenched and mitochondria were lysed in one step by adding SDS in TRIS (pH 8.0 with $\mathrm{HCl}$ ) to an end concentration of $2 \% \mathrm{SDS}$ and $50 \mathrm{mM}$ TRIS. Incubation followed with $10 \mathrm{~min}$ at room temperature. Samples with this treatment were applied directly for SDS-PAGE (2.2.3.2). In case of a favored co-IP analysis, quenching and lysing was achieved in two steps. For this, crosslinking reaction was quenched by adding TRIS (pH 8.0 with $\mathrm{HCl}$ ) to an end concentration of $50 \mathrm{mM}$ for $10 \mathrm{~min}$ at room temperature, mitochondria were reisolated (14 $000 \mathrm{rpm}, 10 \mathrm{~min}, 4^{\circ} \mathrm{C}$ ) and subsequently lysed with solubilization buffer as described in sections 2.2.4.2 and 2.2.4.4, containing 0.3 \% Triton X-100 and $0.1 \%$ SDS to reduce detergent related effects on beads.

\subsubsection{Determination of oxygen consumption rates}

Oxygen consumption was assessed from isolated mitochondria using high resolution respirometry (Oxygraph-2k; Oroboros Instruments, Innsbruck, Austria) in $2 \mathrm{~mL}$ of respiration buffer (Table 2-9) at $30^{\circ} \mathrm{C}$. Samples and all supplements/drugs were added by using syringes (Hamilton) in different sizes (10-50 $\mu \mathrm{l})$ and the different states were determined only from stable signals detected by the oxygen sensor. Wildtype and mutant's oxygen consumptions were measured always in a direct comparison, using both chambers by turns. $50 \mu \mathrm{g} / \mathrm{ml}$ mitochondria were used for all assessments, which was tested before by a premeasurement. Pyruvate $(5 \mathrm{mM})$ and malate $(2 \mathrm{mM})$ were applied to address non-phosphorylating respiration (LEAK). Adding ADP to a saturating concentration (1 $\mathrm{mM})$ (State 3) followed by succinate (10 $\mathrm{mM})$ determined the maximal capacity for coupled oxidative phosphorylation (OXPHOS). By adding antimycin A $(5 \mu \mathrm{M})$ electron transfer from complex III to complex IV was inhibited and respiration was killed. Ascorbate $(2 \mathrm{mM})$ and TMPD $(500 \mu \mathrm{M})$ were added to address OXPHOS capacity specifically via shuttling electrons to complex IV. To distinguish between respiration and auto-oxidation of TMPD/ascorbate, $\mathrm{NaN}_{3}(100 \mathrm{mM})$ was added to block the $\mathrm{O}_{2}$ binding site of complex IV for residual oxygen consumption (ROX), and the values were subtracted from the values after TMPD/ascorbate addition. 


\subsubsection{Determination of $\mathrm{H}_{2} \mathrm{O}_{2}$ production rates}

The assay is following essentially the protocol from Krumschnabel et al. 2015 using a simultaneous approach for measuring mitochondrial $\mathrm{H}_{2} \mathrm{O}_{2}$ production and oxygen consumption (Krumschnabel et al., 2015). It was adapted to be solely performed in cuvettes. Substrates ( $5 \mathrm{mM}$ pyruvate, $2 \mathrm{mM}$ malate, $10 \mathrm{mM}$ succinate) were added to ROS assay buffer. Horseradish peroxidase (HRP, Sigma) $(1 \mathrm{U} / \mathrm{ml}$ ) was used to catalyze fluorescence reaction with $10 \mu \mathrm{M}$ Amplex ${ }^{\mathrm{TM}}$ UltraRed (Invitrogen, in DMSO). Addition of $100 \mu \mathrm{g}$ isolated mitochondria started the reaction, recorded with the spectrofluorometer Xenius (SAFAS) (Excitation $555 \mathrm{~nm}$, slit $10 \mathrm{~nm}$; Emission $581 \mathrm{~nm}$, slit $5 \mathrm{~nm}$ ) in 30 s cycles for $30 \mathrm{~min}$. 


\section{Results}

\subsection{Characterization of Rcf-proteins}

\subsubsection{Rcf-proteins share similar orientation within mitochondria}

Supercomplex or respirasome assembly within the mitochondrial respiratory chain is still elusive regarding the factors which are responsible for attaching the multimeric complexes to each other. In yeast $S$. cerevisiae, especially the Rcf-protein family came into our attention as players in supercomplex assembly and stabilization while the mechanism remains unclear. Rcf1 was shown to be essential for respiration and supercomplex stabilization, however, was also associated with cytochrome $c$ oxidase maturation (Strogolova et al., 2012). Rcf2 has been described to overlap in functions with Rcf1, as well as with Rcf3, possibly linked closely to the homologous regions the proteins harbor (Figure 3-1A) (Strogolova et al., 2012, 2019; Römpler et al., 2016). This study aimed for a better understanding of the distinct and overlapping roles of these factors and enlighten the mechanism of supercomplex assembly. Thus, it was important to bring all three Rcf-proteins in one context and start with a basic characterization of the protein family.

Before focusing on the localization and functionality of the partly homologous domains of the Rcf-proteins, the overall topology was of interest. It was shown before that all three proteins are integrated into the inner mitochondrial membrane (Chen et al., 2012; Vukotic et al., 2012; Römpler et al., 2016; Strogolova et al., 2019). But partly contradictory data exists about the topology of Rcf2 and Rcf1. While Rcf1 was first identified as harboring two transmembrane spans (Chen et al., 2012; Vukotic et al., 2012), a NMR (nuclear magnetic response) analysis revealed five transmembrane spans when expressed in E. coli and reisolated in detergent micelles. The prediction annotates the C-terminus residing in the mitochondrial matrix (Zhou et al., 2018b). The same group determined five transmembrane spans, with the C-terminus exposed to the mitochondrial matrix, by transferring the setup to Rcf2 (Zhou et al., 2018a; Zhou et al., 2020). Yet, in silico analysis identified four transmembrane spans for Rcf2 (Römpler et al., 2016). Since the protein orientation within the mitochondrial membrane depends on the number of transmembrane segments, even or uneven, data respecting the orientation and overall topology of the Rcf-proteins was obtained.

To this end, a protease protection assay was performed in wild-type mitochondria, and proteins of the outer mitochondrial membrane (OMM), intermembrane space (IMS), inner mitochondrial membrane (IMM) and mitochondrial matrix can be distinguished. Always 
accessible for protease treatment, peripheral OMM proteins, like Tom20, are immediately digested and serve as a control for protease treatment. IMS proteins and IMM protein signals are decreased only in swollen mitochondria, losing the OMM as a barrier. However, one has to take the (predicted) topology into consideration and the detectable part of the protein the antibody is binding with. Matrix proteins' signals remain stable throughout the whole experiment, as long as the IMM is intact. Prior solubilization with Triton X-100 provides evidence regarding a general protease digestion ability.

A

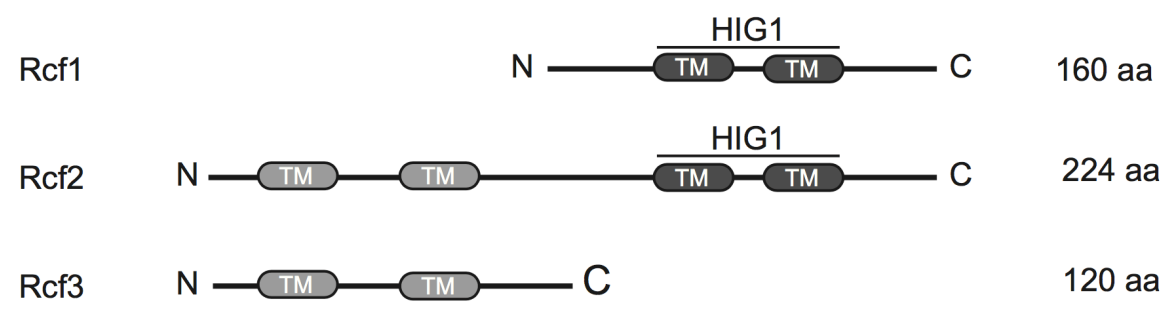

B

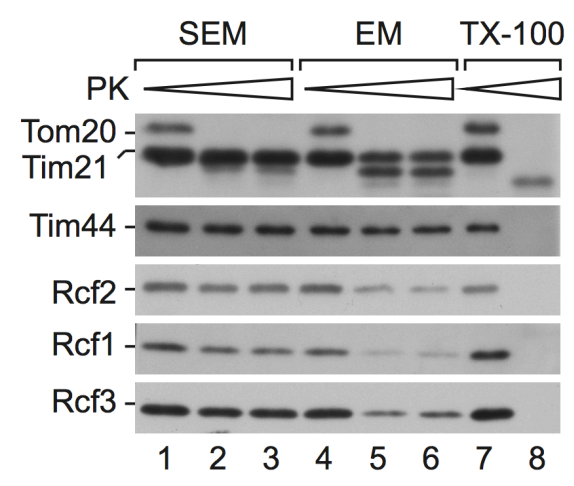

C

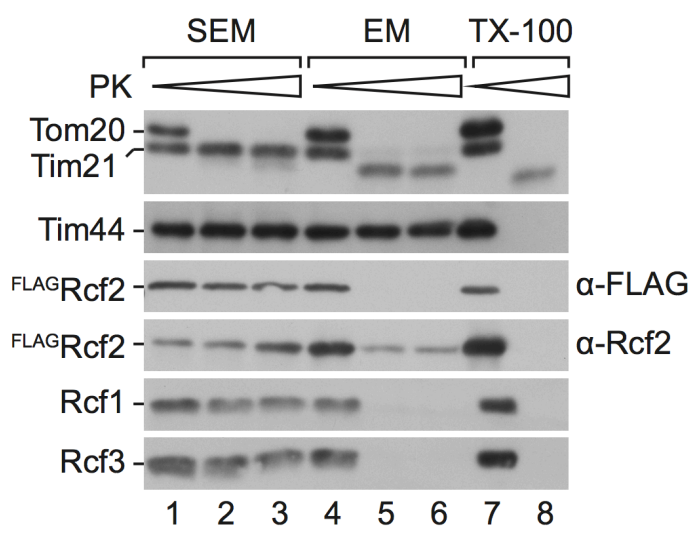

Figure 3-1: Rcf-proteins share homologous domains and similar orientation within mitochondria.

A) Topology model, modified from Römpler et al., 2016. Grey nuances indicate homologous transmembrane spans between the three proteins. HIG1 labels the homology region for hypoxia induced genes. B) Wild-type mitochondria remained intact in isotonic SEM-buffer, converted to mitoplasts in hypotonic EM-buffer or lysed with $1 \%$ Triton X-100, treated with proteinase K (PK; 0-180 $\mu \mathrm{g} / \mathrm{ml}$ ), applied to 10-16 \% SDS-PAGE and western blotting. Tom20, Tim23 and Tim44 served as controls for outer membrane, inner membrane and matrix proteins of mitochondria. C) Isolated mitochondria from $\operatorname{cox} 5 a \Delta r f 2 \Delta$ expressing FLAGRcf2 were subjected to protease protection assay as described in B).

The amounts of Rcf1, Rcf2 and Rcf3 are decreased as soon as the isolated mitochondria are converted into mitoplasts by osmotic swelling. The detected levels remain stable in intact mitochondria (Figure 3-1B). Each serum is raised against the C-terminus of the protein, and we concluded that the C-termini are residing in the IMS. When repeating the experiment with mitochondria from cells expressing ${ }^{\mathrm{FLAG}} \mathrm{Rcf} 2$, it shows an accessible N-terminus, detectable in this case with $\alpha$-FLAG, when the outer mitochondrial membrane is ruptured (Figure 3-1C). This result confirms the idea of Rcf2 as an IMM protein with an even number of transmembrane 
spans as described before (Römpler et al., 2016) and suggests a $\mathrm{N}_{\text {out }}-\mathrm{C}_{\text {out }}$ orientation. Moreover, the experiment indicates that the predicted confirmation of Rcf1 by Zhou et al. (2018b) does not correspond with our in vivo determined situation.

\subsubsection{Rcf-proteins and their influence on respiration}

As a next characterization of the Rcf-proteins we investigated their role in mitochondrial respiration. A Growth-test on fermentable (YPD - glucose) against non-fermentable (YPG glycerol, YPLac - lactate) media is a helpful tool in this regard. While on fermentable media, glycolysis metabolism is able to supply yeast with energy, the oxidative phosphorylation machinery (OXPHOS) has to be fully functional when media are non-fermentable.

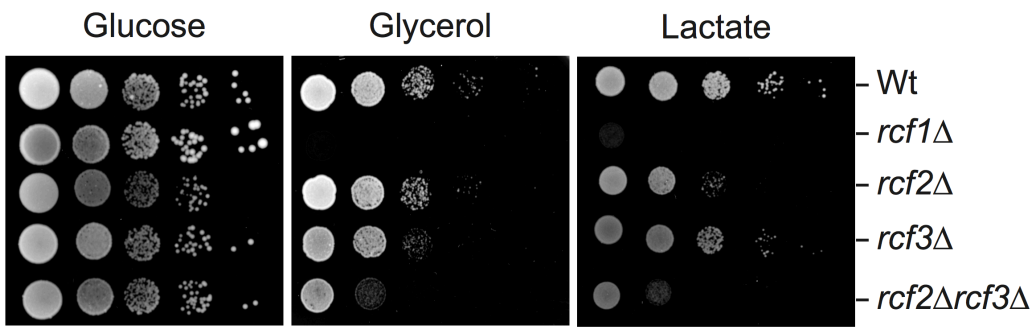

Figure 3-2: Rcf1 is a vital protein for respiration, Rcf2 and Rcf3 only in combination.

Cells of wild-type (Wt), $r f f 1 \Delta, r f 2 \Delta, r f f 3 \Delta, r c f 2 / 3 \Delta$ were spotted on glucose, glycerol, lactate media plates in serial dilution and grown at $37^{\circ} \mathrm{C}$.

$R c f 1 \Delta$ shows heavily affected growth ability when strains are tested on non-fermentable media, whereas $r f 2 \Delta$ and $r f 3 \Delta$ display about wildtype like growth on YPG and YPLac (Figure 3-2). Combining both mutations to a double mutant $\operatorname{rcf} 2 \Delta \operatorname{rrf} 3 \Delta$, the cells cannot cope with the respiratory malfunction and a similar growth phenotype as in $r f 1 \Delta$ appears. This corroborates precedent data of Chen et al. and Vukotic et al., while discussing the relevance of Rcf1 for supercomplex stabilization (Chen et al., 2012; Vukotic et al., 2012). Römpler et al. (2016) showed comparable results for $\operatorname{rrf} 2 \Delta \operatorname{rrf} 3 \Delta$, explaining the overlapping role of the two proteins and ascribed the phenotype to a drop of complex IV activity (Römpler et al., 2016).

Although the RCF1 mutant shows a drastic growth phenotype, protein levels remain more or less stable (Figure 3-3A). Only the complex IV subunits Cox3 and Cox13 show a subtle decrease, other probed cytochrome $c$ oxidase constituents (Cox1, Cox2, Cox12, Cox 5a, Cox5b) were not reduced when isolated mitochondria of the different mutant strains were analyzed on SDS-PAGE. Tested complex III proteins (Cor1, Rip1, Qcr8) did not display any difference in levels as well as the remaining Rcf-proteins. Despite the $r c f 1 \Delta$ resembling growth phenotype of $\operatorname{rcf} 2 \Delta r f f 3 \Delta$, the other RCF mutants did not result in a protein level change. 
Rcf1 and Rcf2 harbor the conserved HIG1 (hypoxia induced gene) domain, and Rcf1 was demonstrated to elevate oxidative stress in cells (Chen et al., 2012). Thus, we monitored the ratio of Cox $5 \mathrm{a}$ and Cox $5 \mathrm{~b}$ to get a reliable prediction of the sensed oxygen status of the cell. Cox $5 \mathrm{a}$ and Cox $5 \mathrm{~b}$ are opposing isoforms, the first being expressed under normoxia and the latter under hypoxia (Hodge et al., 1989). Even though grown under normal growth conditions, the wild-type already shows a basal expression of Cox $5 \mathrm{~b}$. This can be ascribed to a lack of external oxygen supply and culturing in shaking flasks. At the same time, Cox $5 b$ levels remain the same throughout the tested mutants, concluding the Rcf-proteins themselves do not affect the sensed oxygen state of the cells.
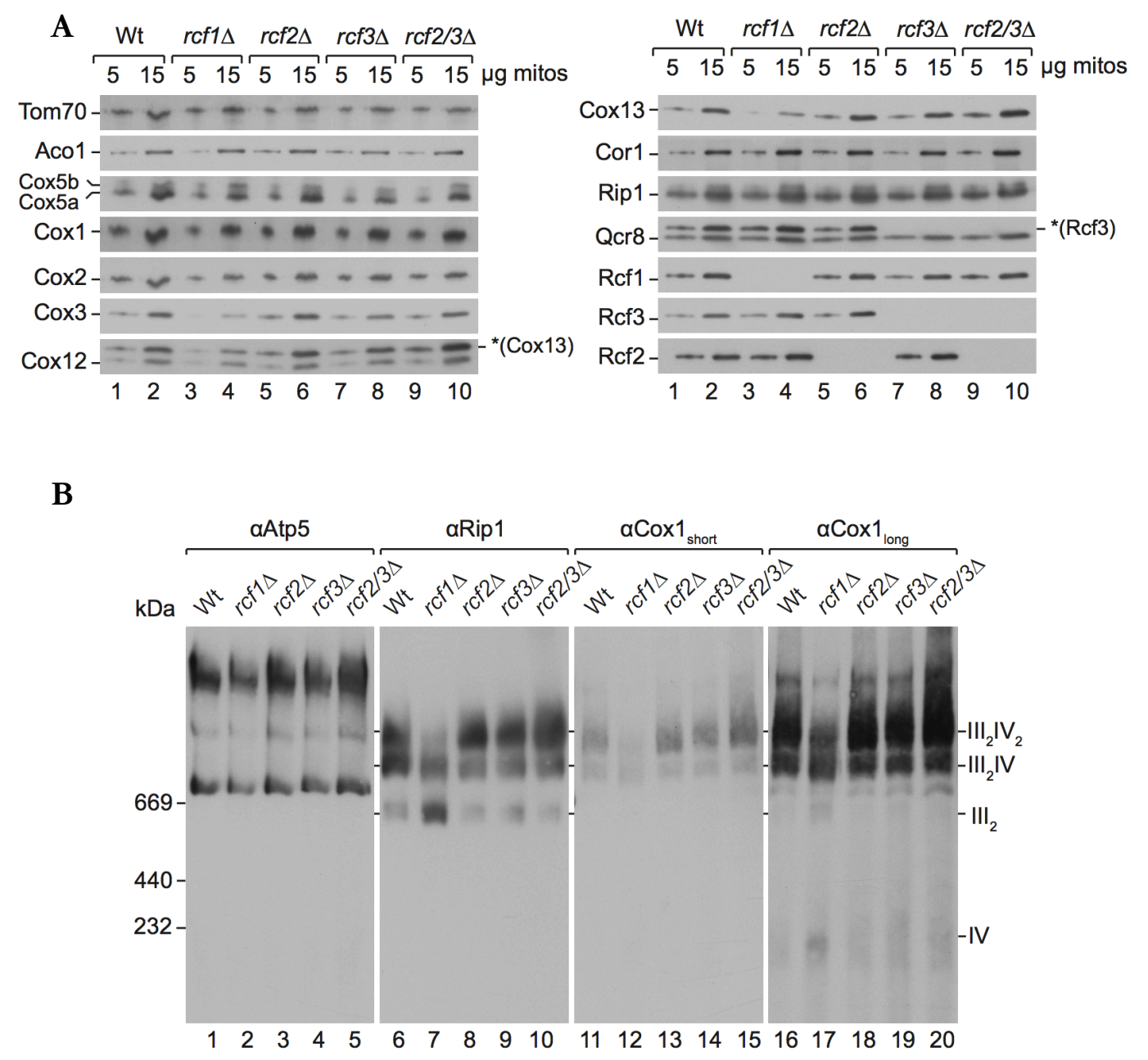

Figure 3-3: Supercomplex assembly is affected in $r c f 1 \Delta$, not reflected by a major decrease in COX subunits.

A) Isolated mitochondria of wild-type (Wt), $r f f \Delta, r f f 2 \Delta, r f f \Delta, r f 2 / 3 \Delta$ subjected to SDS-PAGE (10-16\%) and urea SDS-PAGE (17.5\%) for Cox $5 \mathrm{a} / \mathrm{b}$ separation. Tom70 and Aco1 serve as loading controls. Complex III is analyzed with antibodies against Cor1, Rip1, Qcr8 and complex IV via Cox-antibodies. Asterisks mark protein signals caused by a previous detection with a different antibody. B) Wt, $r f 1 \Delta, r c 2 \Delta, r c f 3 \Delta, r c f 2 / 3 \Delta$ mitochondria solubilized with $1 \%$ digitonin are applied to BN-PAGE (4-10 \%). Atp5 serves as a loading control, detecting complex V. Rip1 reveals complex III and Cox1 complex IV.

Although the protein levels of RCF mutants seem to be ordinary, the formation of supercomplexes could display a different situation given the idea of supercomplex assembly 
factors. The first assessment of respiration capacity additionally implies that the complex association might be disturbed. By BN-PAGE analysis, using the mild detergent digitonin, the structure as respiratory supercomplexes remains intact and the different protein complex formations can be obtained (Schägger and Pfeiffer, 2000). In a wild-type situation, there is a tendency to form the highest molecular respiratory supercomplex $\mathrm{II}_{2} \mathrm{IV}_{2}$, detected in this case with complex IV constituent Cox1 and complex III constituent Rip1 (Figure 3-3B). Additionally, complex $\mathrm{III}_{2} \mathrm{IV}$ and small amounts of complex III dimer can be obtained (Figure 3-3B). The RCF1 mutant shows a clear rearrangement towards $\mathrm{III}_{2} \mathrm{IV}$ and $\mathrm{III}_{2}$, only minor amounts of $\mathrm{III}_{2} \mathrm{IV}_{2}$, and in a longer exposure even free complex IV can be determined. This effect confirms precedent studies, where ref1 caused a rearrangement of respiratory supercomplex (Chen et al., 2012; Vukotic et al., 2012). In parallel, both single and double deletions of $\mathrm{RCF} 2$ and $\mathrm{RCF} 3$ do not lead to a discernible alteration of supercomplex arrangement. The respiratory defect, revealed in the growth test, is not represented by an overall reorganization of the respiratory chain or major compromised protein levels. This was also shown previously by Römpler et al., (2016) arguing Rcf2 and Rcf3 act as more regulatory proteins in respect to the respiratory chain (Römpler et al., 2016).

Overall, Rcf1 caused visible phenotypes on protein level and complex association suggesting furthermore a structural involvement, while the definition of Rcf2 and Rcf3 function is not as obvious. In light of the altered Cox 3 and Cox13 levels, we expected that Rcf1 interacts primarily at this site.

\subsubsection{Rcf-proteins interact with newly synthesized complex IV core subunits}

Previous studies showed an interaction of Rcf1 with newly translated Cox3, reporting a role for complex IV assembly (Strogolova et al., 2012; Su, McStay and Tzagoloff, 2014; Garlich et al., 2017). It remained unclear, however, if the other proteins Rcf2 and Rcf3 may have a similar capacity and we were wondering if we can obtain this by adapting their protocols.

To this end, a mitochondrial translation labeling assay was performed. By inhibiting cytosolic translation with cycloheximide and supplementing with ${ }^{35}$ S-Methionine, it is possible to label exclusively mitochondrial translation products. The generated radioactive proteins are in turn detectable via autoradiogram. In order to conclude specific interactions to Rcf-proteins the Rcf-specific complexes were isolated via immunoprecipitation. In this case, the translation assay was not performed in vivo with whole cells, but with isolated mitochondria to facilitate the 
following immunoprecipitation with the Rcf-protein's antisera. For confirming the specificity of an association with Cox3, the PET494 mutant was included in this examination. Pet494 is a mRNA activator of COX3 and deleting PET494 abolishes COX3 expression without having to mutate the mitochondrial genome itself (Müller et al., 1984; Costanzo and Fox, 1986). To be able to ignore a possibly occurring supercomplex assembly, DDM was utilized as a detergent, disrupting complex $\mathrm{III}_{2} \mathrm{IV}_{(1-2)}$ association.
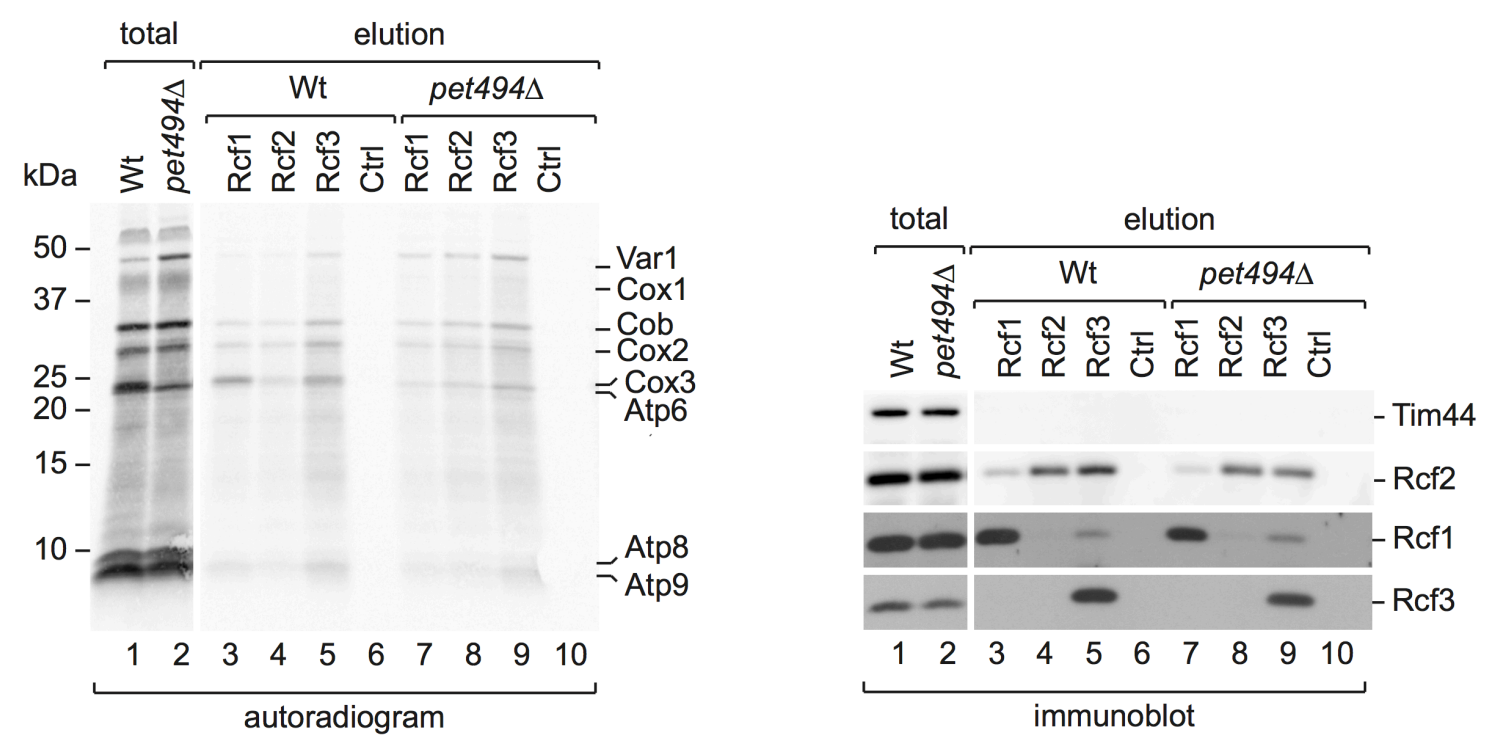

Figure 3-4: Rcf-proteins co-isolate mitochondrial encoded proteins.

Isolated wild-type $(\mathrm{Wt})$ and pet494 $\Delta$ mitochondria are subjected to radioactive labeling of mitochondrial encoded proteins with ${ }^{35} \mathrm{~S}$-methionine for $10 \mathrm{~min}$, followed by solubilization with 0.6 \% DDM and co-immunoprecipitation with Rcf1, Rcf2, Rcf3 and control beads. Totals (5\%) and glycine elution (100\%) were subjected to Tricine-SDS PAGE (10-18\%), western blotting, autoradiogram and subsequently immunodetection. Tim44 serves as a control for specific complex isolation.

Eliminated COX3 translation does not interfere with translation of the other seven mitochondrially encoded proteins, and their radioactive signals remained stable (Figure 3-4). We could determine that Rcf1 most dominantly interacts with Cox3 in a wild-type situation, compared with the other labeled proteins. This gets in line with previous results from Strogolova et al. (2012) and Garlich et al., (2017) showing an interaction in Triton solubilized mitochondria of His-tagged Rcf1 with radioactive labeled Cox3 (Strogolova et al., 2012; Garlich et al., 2017). The Rcf-protein were demonstrated to interact with each other under digitonin solubilizing conditions. DDM solubilization however, previously showed that the Rcf-protein interaction is labile and the proteins easily dissociate under these conditions (Römpler et al., 2016). Although utilizing DDM for solubilization in our experiment, small amounts of Rcf2 can be co-isolated proceeding the isolation from Rcf1 (Figure 3-4). In parallel, this does not apply vice versa for immunoprecipitation with Rcf2. 
Surprisingly, Rcf2 and Rcf3 could co-isolate mitochondrial translation products as well, which was not part of previous studies. Along with Rcf2 only minor amounts of newly translated Cox 3 are co-purified, while Rcf3 brings down almost comparable amounts as Rcf1. Overall, Rcf2 shows the lowest efficiency in immunoprecipitation under these conditions considering bait protein levels, whereas Rcf3 co-purifies both Rcf2 and Rcf1.

Nevertheless, other mitochondrial encoded proteins like Cox2 and Cob, even ATPase constituents can be isolated along the three antibodies. Interaction with Cox2, also displayed in previous studies (Strogolova et al., 2012; Garlich et al., 2017) argues for already matured cytochrome $c$ oxidase in interaction with the Rcf-proteins. Cob isolation speaks for a parallel interaction with complex III independent of complex IV under these circumstances.

The association of not only Rcf1 but also Rcf2 and Rcf3 with newly synthesized Cox3 and Cox 2 was indicative for an interaction site of the proteins. Yet, we were aiming for a more detailed characterization of interactors since the functional mechanism of Rcf-proteins is still obscure. Considering that the Rcf-proteins are suggested as transient interactors of complex IV (Vukotic et al., 2012; Römpler et al., 2016; Garlich et al., 2017), we aimed for catching those associations by chemical crosslinking. Combined with mass-spectrometric analysis, performed with collaborators (Linden et al., 2020), this represents a high-quality screening method.

\subsection{Crosslinks reveal Rcf2 and Rcf3 in close interaction with COX subunits}

The Rcf-proteins were identified as they are able to interact independently with complex IV and complex III (Vukotic et al., 2012; Römpler et al., 2016). In parallel, it is proven that they associate with the respiratory supercomplexes and can be isolated along with them (Chen et al., 2012; Strogolova et al., 2012; Vukotic et al., 2012; Römpler et al., 2016). In earlier cryo-EM structures obtained from yeast respiratory supercomplex $\mathrm{III}_{2} \mathrm{IV}_{(1-2)}$, none of the three Rcf proteins could be resolved (Mileykovskaya et al., 2012; Hartley et al., 2019; Rathore et al., 2019), arguing for a substoichiometric interaction. A proposed interacting site at the interface of complex IV and complex III as supercomplex assembly factors (Vukotic et al., 2012) stands in contrast to the influences on the assembly of the peripheral proteins Cox13 and Cox12 (Vukotic et al., 2012; Strogolova et al., 2019). However, Hartley and colleagues just recently identified Rcf2 $2^{107-205}$ as a peripheral constituent of supercomplex $\mathrm{III}_{2} \mathrm{IV}$ in a cryo-EM analysis (Hartley et al., 2020). Here, they isolated the complex with the help of $6 x H$ is tagged Cox13 in a cox5a background to 
enhance homogeneity of the complex (see section 3.2.1). This, on the other hand, suggests a stoichiometric interaction of Rcf2 with the supercomplex at least under these conditions and questions the idea of Rcf2 as an assembly factor.

Consequently, it was an interesting finding when we identified Rcf2 and Rcf3 together with collaborators in mass spectrometry analysis of crosslinked mitochondria (Linden et al., 2020). We used the chemical, homobifunctional crosslinker $\mathrm{BS}^{3}$, harboring a linker length of $11.4 \AA$. $\mathrm{BS}^{3}$ links primary amino groups by reacting as a sulfo-NHS ester (N-hydroxysuccinimide), consequently, N-termini of polypeptide chains and the sidechains of lysine are targeted. By utilizing whole mitochondria, only a fraction of crosslinked proteins originated from the respiratory chain. Yet, the advantage was that the data represented in vivo behavior of the proteins.

We could determine the $\mathrm{N}$-terminus of Cox 12 crosslinked to the $\mathrm{N}$-terminus of Rcf3, as well as the C-terminal region of Rcf2 (Figure 3-5A) (Linden et al., 2020). In parallel, the same amino acid of Rcf2 was found to crosslink with a C-terminal region of Cox13 (Figure 3-5A). Interestingly, no crosslinks were obtained within the Rcf-family under those conditions, in regard of the proteins interacting with each other (Römpler et al., 2016). In parallel, Rcf1 was not identified as crosslinked to respiratory chain complexes (Linden et al., 2020), although a similar localization was suggested earlier as for Rcf2 (Strogolova et al., 2019; Dawitz et al., 2020).

A

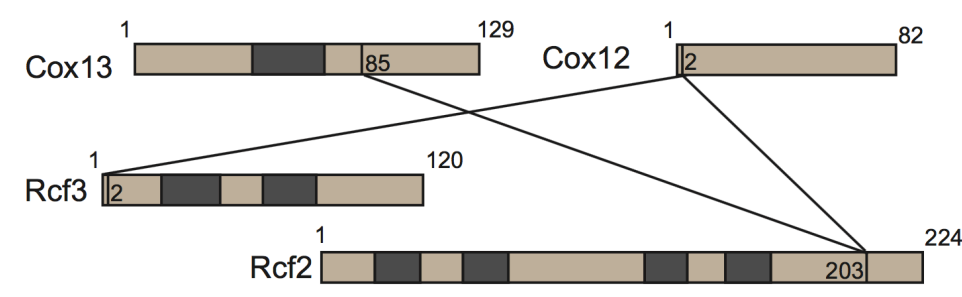

B

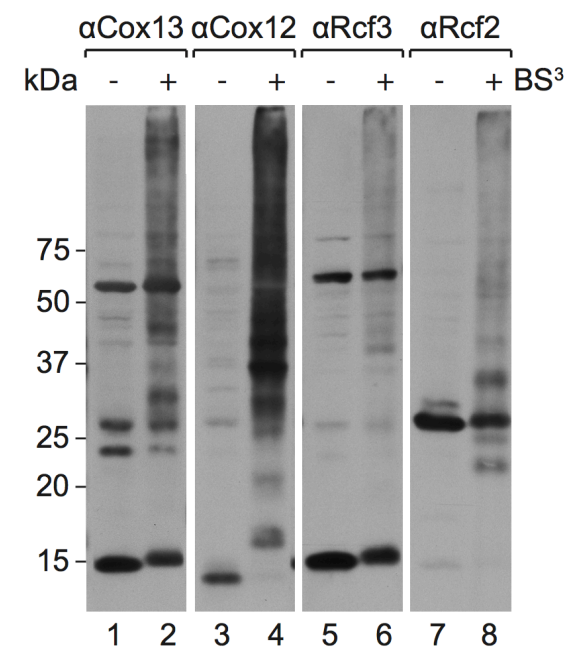

Figure 3-5: Rcf2 and Rcf3 are crosslinked to Cox12 and Cox13.

A) Model representing the mass spectrometry results of whole mitochondria subjected to crosslinking with $\mathrm{BS}^{3}$ reduced to Rcf-protein data, as published in Linden et al. (2020). Numbers mark the crosslinked amino acids connected by lines. Dark grey areas display putative transmembrane segments. B) Isolated wild-type mitochondria were crosslinked with $50 \mu \mathrm{M} \mathrm{BS} 3$ for $1 \mathrm{~h}$, subsequently quenched and lysed with $2 \%$ SDS in TRIS (pH 8.0), applied to SDS-PAGE (10-16\%) and western blotting. Control samples without added crosslinker went through the same procedure in parallel. 
In order to confirm this mass spectrometry data, we performed a crosslinking assay, using the same protocol as in Linden et al. (2020). Isolated wild-type mitochondria were subjected to crosslinking with $\mathrm{BS}^{3}$, quenched with TRIS and lysed with SDS. We first analyzed the general crosslink pattern by probing for the different candidates and comparing samples with and without crosslinker (Figure 3-5B). Prominent crosslinks for Cox13 could be determined at around $30 \mathrm{kDa}, 40 \mathrm{kDa}$ and $60 \mathrm{kDa}$. Cox12 showed specific signals around $18 \mathrm{kDa}, 21 \mathrm{kDa}$, $30 \mathrm{kDa}$, and a strong crosslinking blur appeared above $37 \mathrm{kDa}$. For Rcf3, only low amounts of crosslinks at $30 \mathrm{kDa}$ and around $40 \mathrm{kDa}$ could be detected. Whereas for Rcf2, stronger signals were detectable at approximately $30 \mathrm{kDa}$ and $40-50 \mathrm{kDa}$.

\subsubsection{Rcf2 crosslinks specifically to Cox12 and Cox13}

To be able to uncover specific crosslinks for the different proteins biochemically, co-immunoprecipitation with antisera against Rcf2, Cox13 and Cox12 was carried out. To this end, the experiment was executed in the same way as before, except mitochondria were solubilized with low concentrated Triton X-100 and SDS. Any native interaction should be disrupted under these conditions and only crosslinked protein complexes were co-purified, still providing appropriate conditions for efficient antigen-antibody reactions on coated beads.

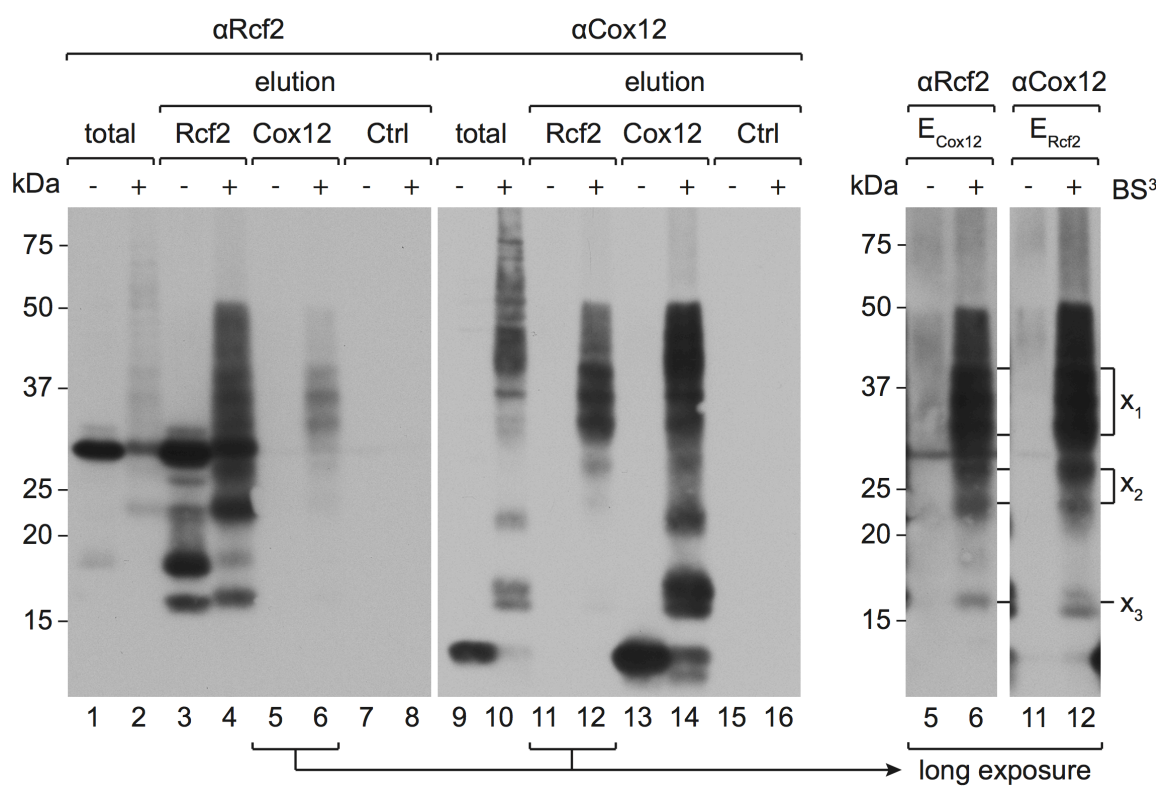

Figure 3-6: Rcf2 specifically crosslinks with Cox12.

Wild-type mitochondria were subjected to crosslinking with $50 \mu \mathrm{M} \mathrm{BS} 3$ for $1 \mathrm{~h}$, reaction was quenched with TRIS, mitochondria were lysed with $0.5 \%$ Triton and $0.1 \%$ SDS, and applied to co-immunoprecipitation with Rcf2, Cox12, control beads. Totals and glycine eluates were used for SDS PAGE (10-16\%) and western blotting. Signals were detected with Rcf2 and Cox12 antibodies, respectively. Control samples without added crosslinker went through the same procedure in parallel. $\mathrm{x}_{1}, \mathrm{x}_{2}$ and $\mathrm{x}_{3}$ mark specific crosslinks between Rcf2 and Cox12. 
The respective antibody against the opposite, crosslinked protein was used for detection and vice versa, in order to identify the definite association. In fact, different crosslinks or crosslink-regions could be purified and detected by this approach (Figure 3-6).

A crosslinking ladder at $\sim 30-40 \mathrm{kDa}\left(\mathrm{x}_{1}\right)$, another one right beneath at $\sim 21-28 \mathrm{kDa}\left(\mathrm{x}_{2}\right)$ and one specific crosslink at $\sim 16 \mathrm{kDa} \quad\left(\mathrm{x}_{3}\right)$ were isolated with both, Cox12 and Rcf2 co-immunoprecipitation, and detected with the particular other antibody. This indicates a specific appearance, although full-length Rcf2 $(25 \mathrm{kDa})$ and Cox12 $(10 \mathrm{kDa})$ can only be responsible for the crosslinks $\mathrm{x}_{1}$, according to their size. $\mathrm{X}_{2}$ might occur due to a processed version of Rcf2, which is detectable in enriched quantity after Rcf2 immunoprecipitation (Figure 3-6, lane 3).

An internal processing event of Rcf2 was studied previously by Römpler et al. (2016) resulting in a stable $\mathrm{Rcf} 2^{\mathrm{C}}$ fragment $(\sim 21 \mathrm{kDa})$ (Römpler et al., 2016). This corresponds to the fragment detected at $\sim 18 \mathrm{kDa}$ in this case. This minor discrepancy can be justified with the usage of different gel systems and standard reference markers. It has to be noted, however, that another fragment can be enriched as well at $\sim 16 \mathrm{kDa}$, which is not represented in previous published data (Römpler et al., 2016). In contrast to the slightly larger fragment, that fragment is not detectable in the total and could be an artefact caused by the procedure itself.

Upon addition of the molecular weight of $\operatorname{Rcf}^{\mathrm{C}}$ and Cox12 to $\sim 28 \mathrm{kDa}$, it can be conceived that those partners generate the isolated crosslink $\mathrm{x}_{2}$. The obtained crosslink $\mathrm{x}_{3}$ however, is only $\sim 7 \mathrm{kDa}$ larger than Cox12 and the interacting partner remains unknown.

In order to identify the crosslinks of Rcf2 and Cox13, we used the same Rcf2 elution fraction for a second SDS-PAGE, loaded next to the Cox13 bound fraction (Figure 3-7). By this, the detection can occur from the same gel or membrane and mutual crosslinks can be specified with higher certainty. At first sight, the isolation of crosslinks between these two proteins appears to be less efficient than before with Cox12. Although general co-immunoprecipitation capacity seems to be at a satisfying level (Figure 3-7, lanes 3 and 13), the detection with the respective other antibody was weak (Figure 3-7, lanes 6 and 12). Paying attention to longer exposures of the corresponding lanes $(5,6,11$ and 12), we could obtain two crosslinks, corresponding the two proteins by size. Full-length Rcf2 $(25 \mathrm{kDa})$ and Cox13 (15 kDa) can be added up to $40 \mathrm{kDa}$, which is roughly where $x_{1}$ is detected. Considering again the $\operatorname{Rcf} 2^{C}$ fragment at $\sim 18 \mathrm{kDa}$, the other crosslink $\mathrm{x}_{2}$ finds an explanation. However, there is a third crosslink $\mathrm{x}_{3}$ of unknown nature isolated, as well $\sim 7 \mathrm{kDa}$ larger than the protein of interest, Cox13. Once more, a smaller fragment of Rcf2 could be detected, also present in the Cox13 elution. One has to note, that an 
obvious amount of unlinked Cox13 can be determined upon Rcf2 co-immunoprecipitation (lane 12) but only under crosslinking conditions. Arguing for a tight interaction of the two proteins, this should have been detectable in the sample without crosslinker as well. It could also indicate a strong association of Cox13 to another Rcf2 crosslinked protein which was not identified by mass spectrometry.

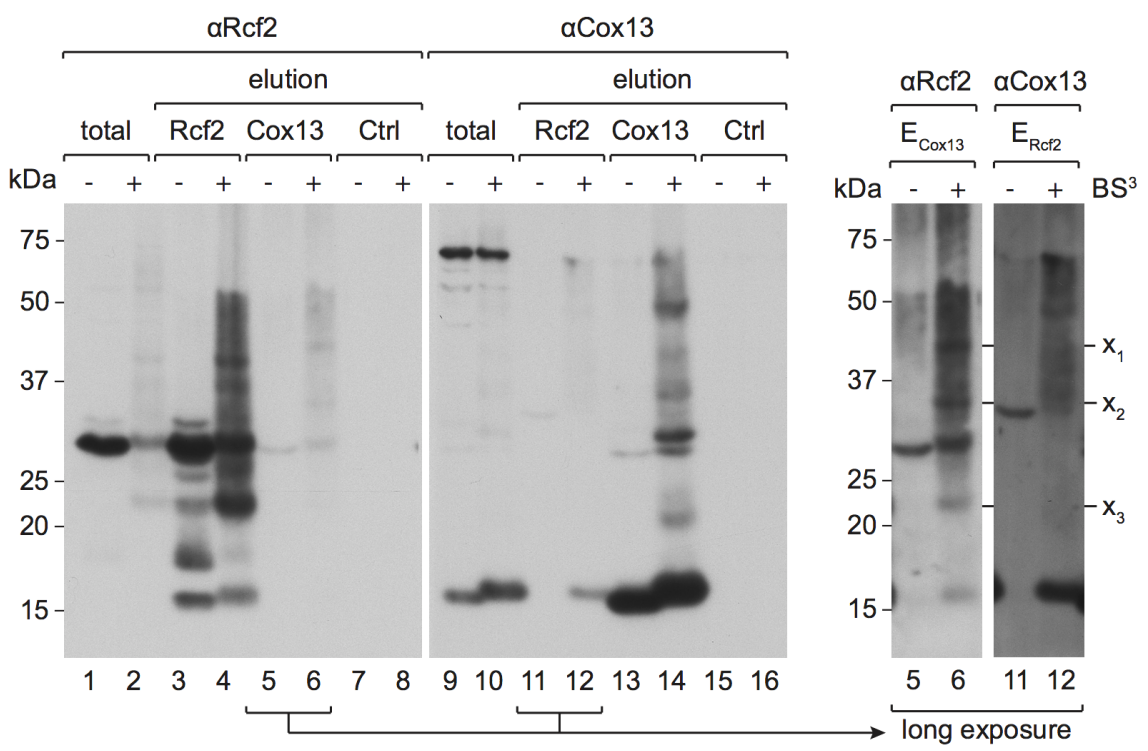

Figure 3-7: Rcf2 specifically crosslinks with Cox13.

Wild-type mitochondria were subjected to crosslinking with $50 \mu \mathrm{M} \mathrm{BS} 3$, reaction was quenched with TRIS, mitochondria were lysed with $0.5 \%$ Triton and $0.1 \%$ SDS and applied to co-immunoprecipitation with Rcf2, Cox 13 , control beads. Totals and glycine eluates were used for SDS PAGE (10-16 \%) and western blotting. Signals were detected with Rcf2 and Cox13 antibodies, respectively. Control samples without added crosslinker went through the same procedure in parallel. $\mathrm{x}_{1}, \mathrm{x}_{2}$ and $\mathrm{x}_{3}$ mark specific crosslinks between Rcf2 and Cox13.

In the end, those identified crosslinks are consistent with the idea of Rcf2 as interacting with the peripheral part of the oxidase. The recently published cryo-structure of complex $\mathrm{III}_{2} \mathrm{IV}$ resolved Rcf2 $2^{107-205}$ in association with the respirasome where it sits between Cox13 and Cox3 clamped right below Cox12 (Figure 3-8A) (Hartley et al., 2020). This structure not only helped to confirm the proposed crosslinks by analyzing the distances but also indicates in which state the crosslinks occurred. In fact, the distance between Cox12-K41 and Rcf2-K203 measures 11.6 $\AA$, which corresponds well to the linker length of $\mathrm{BS}^{3}$ of $11.4 \AA$ (Figure $3-8 \mathrm{~B}$ ). This implies that the crosslinked Rcf2 is positioned at the supercomplex not only the cytochrome $c$ oxidase itself. It is also interesting to note, that exclusively the C-terminus is resolved in this cryo-structure and it appears that also the $\operatorname{Rcf}^{\mathrm{C}}$ fragment itself can be found crosslinked to Cox12. Since processed Rcf2 is not part of a protein database for mass-spectrometry calculation, 
the only evidence is our biochemical analysis. Yet, it indicates that not only full-length Rcf2 but also Rcf $2^{C}$ is still in proximity to Cox12 and Cox13.

Analyzing the crosslink observed between Rcf2-K203 and Cox13-K85 with the help of the available cryo-structure however, resulted in a distance of $60.5 \AA$ (Figure 3-8C). It is known that the theoretical crosslink linker length can differ from the actual distance. It was shown that $\mathrm{BS}^{3}$ crosslinks up to $30 \AA$ distant lysine chains due to dynamic reasons (Merkley et al., 2014), but the distance between Rcf2 and Cox13 exceeds this threshold twice as much. This suggests that the crosslink between those chains does not take place at the endpoint represented by cryo-EM and might rather be a result of a dynamic interaction of Rcf2 with the cytochrome $c$ oxidase.

A

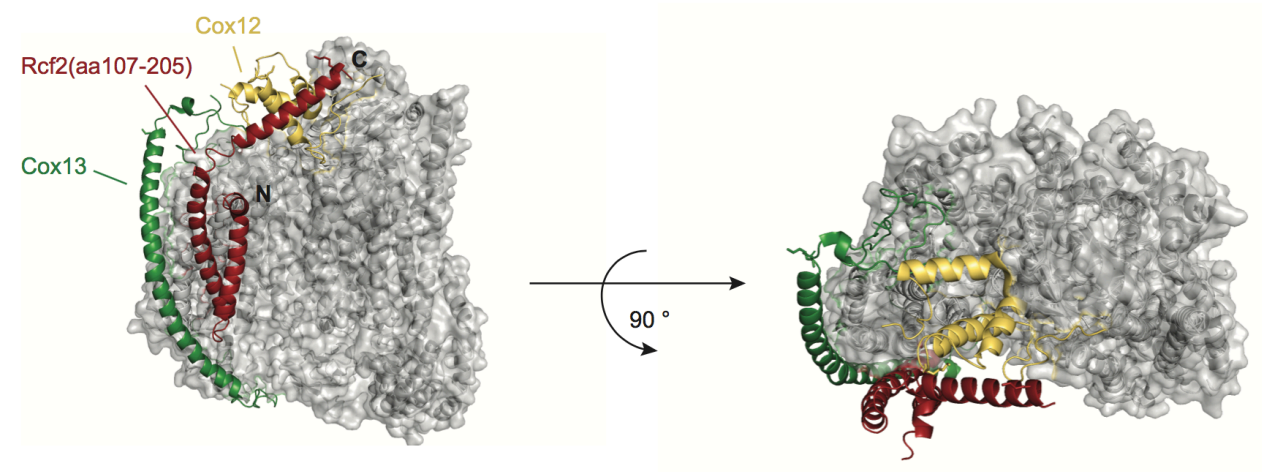

B

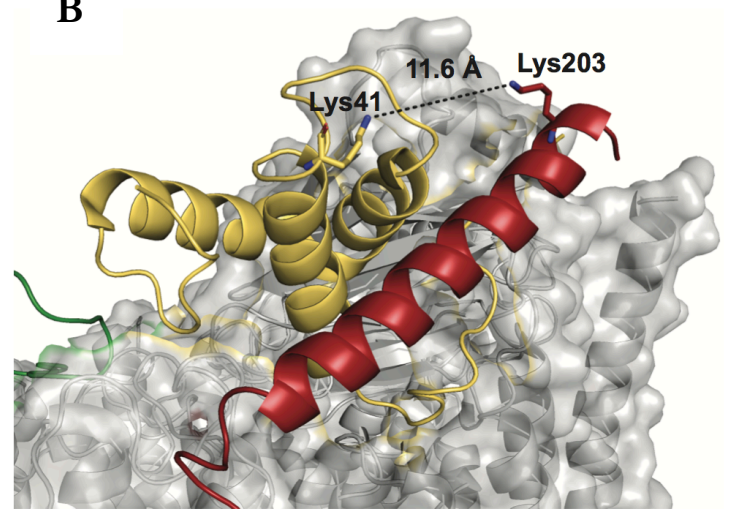

C

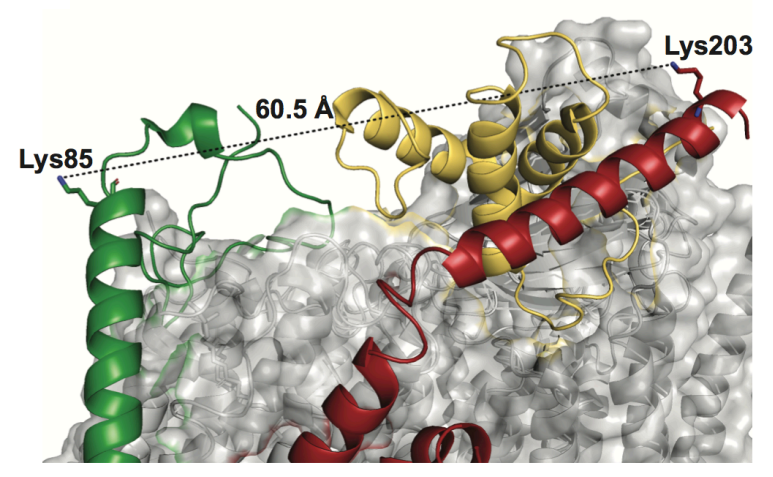

Figure 3-8: Structural analysis reveals crosslink of Rcf2 and Cox12 likely to happen at the supercomplex.

A) Localization of Rcf2 at cytochrome c oxidase extracted from cryo-EM structure of Hartley et al. (2020) (PDB ID 6T15). Dark red: Rcf2, green: Cox13, yellow: Cox12, grey surface: remaining COX subunits B) and C) Crosslink between lysine sidechain K203 of Rcf2 (dark red) and lysine sidechain K41 of Cox12 (yellow) or lysine sidechain K85 of Cox13 (green), respectively. The dashed line indicates the suggested crosslink. Analysis and editing were performed with Pymol software.

\subsubsection{Rcf3 specifically crosslinks to Cox12}

In addition to Rcf2, Rcf3 was also found to crosslink with Cox12. In the interest to confirm this data, we adopted the same experimental setup as described before (section 3.2.1) to Rcf3 
co-immunoprecipitation. As previously, eluates of the complexes were probed with the respective other antibody to verify mutual crosslinks. The only considerable enriched signals appeared at $\sim 30 \mathrm{kDa}\left(\mathrm{x}_{1}\right)$ and $\sim 23 \mathrm{kDa}\left(\mathrm{x}_{2}\right)$. Both roughly correspond the added size of the proteins $14 \mathrm{kDa}$ for $\mathrm{Rcf} 3$ and $10 \mathrm{kDa}$ for Cox12. Since $\mathrm{x}_{2}$ is enriched to a greater extent, this crosslink was assumed as characteristic for Rcf3 and Cox12. Other signals, detected in higher molecular weight regions were identified as unspecific since they were detectable in both samples, with or without crosslinker.

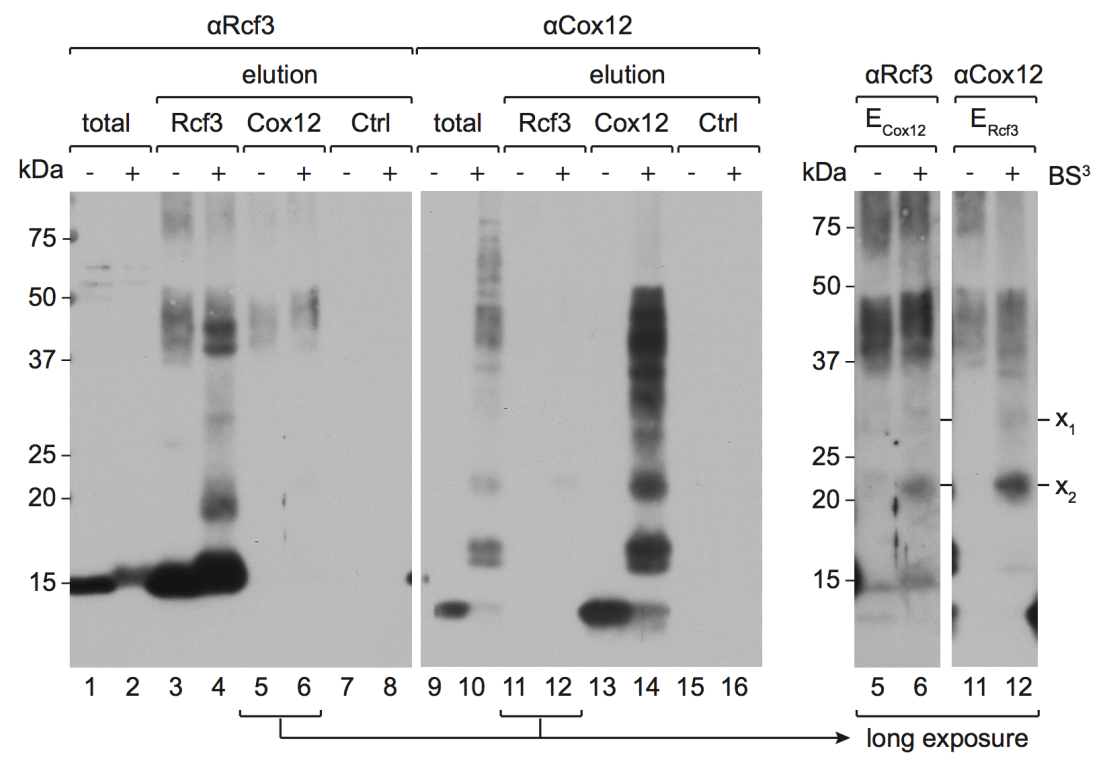

Figure 3-9: Rcf3 specifically crosslinks with Cox12.

Wild-type mitochondria were subjected to crosslinking with $50 \mu \mathrm{M} \mathrm{BS}{ }^{3}$ for $1 \mathrm{~h}$, reaction was quenched with TRIS, mitochondria were lysed with $0.5 \%$ Triton and $0.1 \%$ SDS and applied to co-immunoprecipitation with Rcf3, Cox12, control beads. Totals and glycine eluates were used for SDS PAGE (10-16\%) and western blotting. Signals were detected with Rcf3 and Cox12 antibodies, respectively. Control samples without added crosslinker went through the same procedure in parallel. $\mathrm{x}_{1}$ and $\mathrm{x}_{2}$ mark specific crosslinks between Rcf3 and Cox12.

Rcf3 shares homologies with the N-terminus of Rcf2 and was shown to overlap in functions (Römpler et al., 2016). Thus, it could be expected that it is located at a similar interaction site as Rcf2. Although Rcf3 is not resolved in the cryo-structure (Hartley et al., 2020), we could still conclude an appropriate position from our results (Figure 3-8A). Considering the linker length of $\mathrm{BS}^{3}(11.4 \AA)$, it is likely that the crosslink of Rcf3 and Cox12 occurs next to Rcf2, but there is no indication whether the crosslink happens at the level of supercomplexes. Nevertheless, we provided the novel evidence for Rcf3 interacting with the peripheral cytochrome $c$ oxidase. 


\subsection{Min8 - a novel complex IV interactor}

In context of the crosslinking analysis, it was intriguing to discover the so far uncharacterized protein Min8 (YPR10C-A) crosslinked to Cox12 (Figure 3-10A) and Pet9 (Aac2). The small protein of 72 amino acids was part of mitochondrial proteome studies of Morgenstern et al. (2017) and identified as a single span IM protein but not included in further investigations. Re-evaluation of previous mass-spectrometry results indicate an association of Min8 with the respiratory supercomplex, although a relation to complex IV could not be specified (Vukotic et al., 2012).

In order to analyze the association of Min8 at complex IV, the previous biochemical analysis of crosslinks (see 3.2.1 and 3.2.2) was transferred to this purpose as well. A HA-tagged version of Min8 was utilized to facilitate Min8 detection, but also co-immunoprecipitation. First, an overall pattern of the crosslinks of Min8-HA was obtained in direct comparison with the wild-type and the respective antibody detection (Figure 3-10B).

A

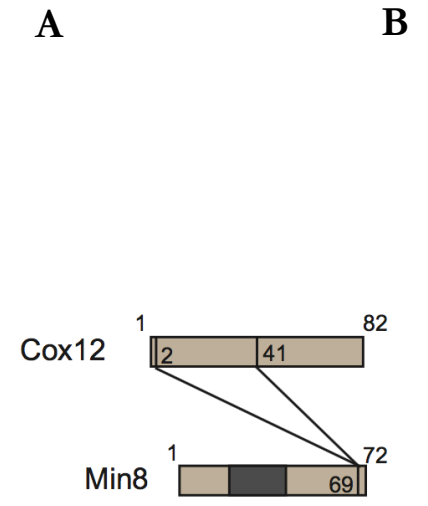

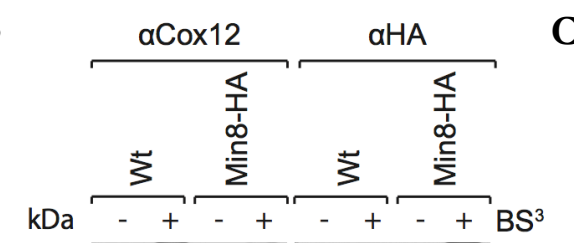
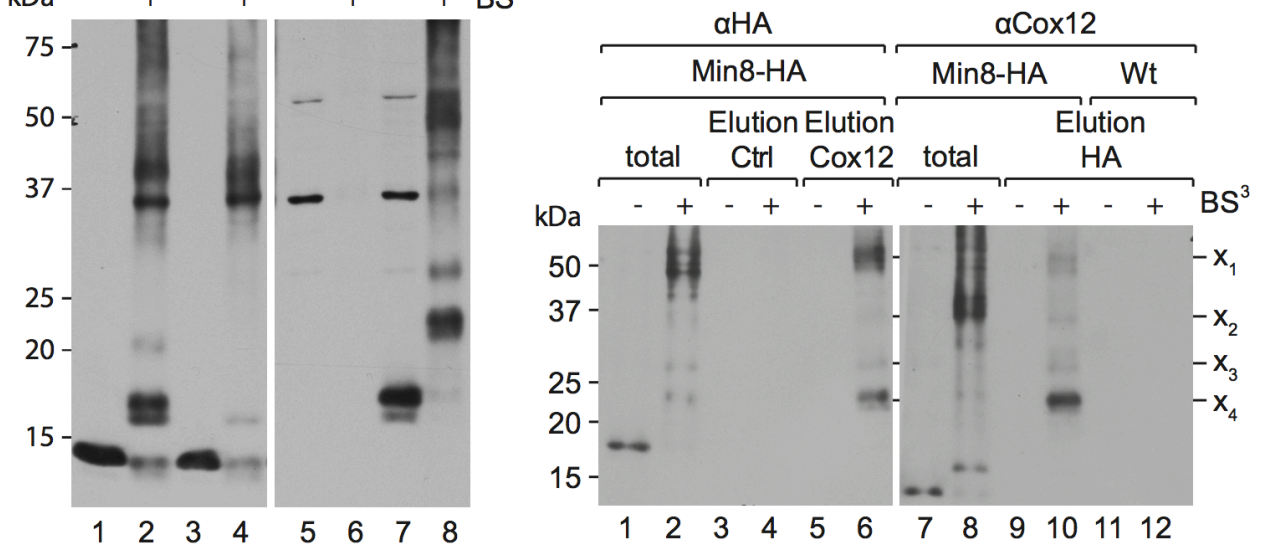

Figure 3-10: The uncharacterized protein Min8 (YPR010C-A) is specifically crosslinked to Cox12.

A) Model representing the mass spectrometry results of whole mitochondria subjected to crosslinking with BS $^{3}$ reduced to Cox12 and Min8, as published in Linden et al. (2020). Numbers mark the crosslinked amino acids connected by lines. Dark grey area displays the transmembrane segment. B) Isolated wild-type and Min8-HA mitochondria were treated with $50 \mu \mathrm{M} \mathrm{BS}{ }^{3}$ for $1 \mathrm{~h}$, reaction was quenched with TRIS. Control samples without added crosslinker went through the same procedure in parallel. Mitochondria were lysed with $2 \%$ SDS, applied to SDS-PAGE (10-16\%) and western blotting. Cox12 and HA antibody were used for detection. C) Crosslinking procedure was performed as in B). After quenching reaction, mitochondria were solubilized with $0.5 \%$ Triton and $0.1 \%$ SDS and applied to co-immunoprecipitation with Cox 12 , HA and control beads. Totals and glycine eluates were used for SDS-PAGE (10-16\%) and western blotting. Signals were detected with Cox12 and HA antibodies, respectively. Wild-type samples served as a control for HA isolation. $\mathrm{x}_{1}, \mathrm{x}_{2}, \mathrm{x}_{3}$ and $\mathrm{x}_{4}$ mark specific crosslinks between Min8 and Cox12 
Examining the two samples, it is striking that a Cox12 crosslink around $18 \mathrm{kDa}$ disappears in Min8-HA, compared to the wild-type (Figure 3-10B lanes 2, 4). The same antibody fails to detect a crosslink within the Min8-HA strain, shifted by the corresponding size of the $3 \mathrm{xHA}$ $\operatorname{tag}(3 \mathrm{kDa})$. Still, it implies that this is the specific crosslink between the two proteins.

As performing isolation via Cox12 and the HA-tag in a Min8-HA strain however, a crosslink at $\sim 22 \mathrm{kDa}\left(\mathrm{x}_{4}\right)$ could be enriched specifically (Figure 3-10C). Adding up the molecular mass of Cox12 $(10 \mathrm{kDa})$ and Min8-HA $(8+3 \mathrm{kDa}$ for $3 \mathrm{xHA})$, this prominent crosslink was conceived as the specific crosslink between Min8-HA and Cox12. Additionally, other crosslinks at $\sim 28 \mathrm{kDa}\left(\mathrm{x}_{3}\right), \sim 36 \mathrm{kDa}\left(\mathrm{x}_{2}\right)$ and $\sim 50 \mathrm{kDa}\left(\mathrm{x}_{1}\right)$ could be obtained by this approach. The mass spectrometry analysis revealed two amino acids of Cox12 to be crosslinked with Min8. Additionally to aa2 which was crosslinked with other proteins, also aa41 (Figure 3-10A) (Linden et al., 2020). This leads to the assessment that both isolations could purify Cox12-Min8 in parallel to crosslinks of Cox12 with other candidates. Considering the mass estimation from the western blot, there are shifts of $+6 \mathrm{kDa},\left(\mathrm{x}_{3}\right)+14 \mathrm{kDa}\left(\mathrm{x}_{2}\right)$ and $+28 \mathrm{kDa}\left(\mathrm{x}_{1}\right)$ additionally to the putative Cox12-Min8-HA ( $\left.\mathrm{x}_{4}\right)$ crosslink. These correspond roughly the identified crosslinked proteins of Cox12; $\operatorname{Cox} 9$ (7 kDa), Rcf3 (14 kDa) and Rcf2 (25 kDa). Thus, it allows the assumption that all these proteins are isolated together in the same experiment. This idea could also fit to the unidentified crosslinks detected in the experiments before with Rcf2 ( $x_{3}$ in Figure 3-6, section 3.2) and Rcf3 ( $\mathrm{x}_{1}$ in Figure 3-9, section 3.2).

In the end, Min8 can be positioned in proximity to Rcf2 but also Rcf1, considering a reported interaction of Rcf1 with Aac2 and Cox3 (Strogolova et al., 2019). It is likely that the Min8-Cox12 interaction is happening on a respiratory supercomplex basis, given the evidence from previous mass-spectrometry analyses (Vukotic et al., 2012). Acknowledging the mutual interacting partners, it was tempting to conceive Min8 as a novel respiratory supercomplex factor. In order to gather more information about this proposition, Min8 was examined in regard of its involvement in supercomplex formation and respiration.

\subsubsection{Influence of Min8 in respiration}

To investigate the role of Min8 in respect of a potential connection to the Rcf-proteins, a double deletion strain was created in addition to $\min 8 \Delta$ : $\min 8 \Delta r c f 2 \Delta$. Both proteins were found to localize adjacent to each other and the idea was that a specific phenotype could arise from a double knockout. 
In a steady state analysis of the respective strains' mitochondria, no discernible difference could be detected, comparing especially complex IV (Cox1, Cox12, Cox13, Cox5a/b) and complex III (Cor1, Rip1) subunits (Figure 3-11A). Also, Rcf-protein levels did not differ between wild-type, $\min 8 \Delta, r c 2 \Delta$ and $\min 8 \Delta r r f 2 \Delta$. Similarly, the oxygen sensed state of the cell, determined by the ratio of the normoxic/hypoxic isoforms Cox 5a and Cox5b, remained stable (Figure 3-11B). As a following characterization, the cellular respiration capacity was evaluated by performing a growth drop-test on non-fermentable media. Neither min $8 \Delta$ nor $\min 8 \Delta \operatorname{rcf} 2 \Delta$ did develop a growth defect on non-fermentable carbon sources (Figure 3-11C). The double mutant rather displayed an enhanced growth ability but on both, glucose and glycerol media. Consequently, Min8 is not considered vital for respiration, neither in combination with Rcf2.

A
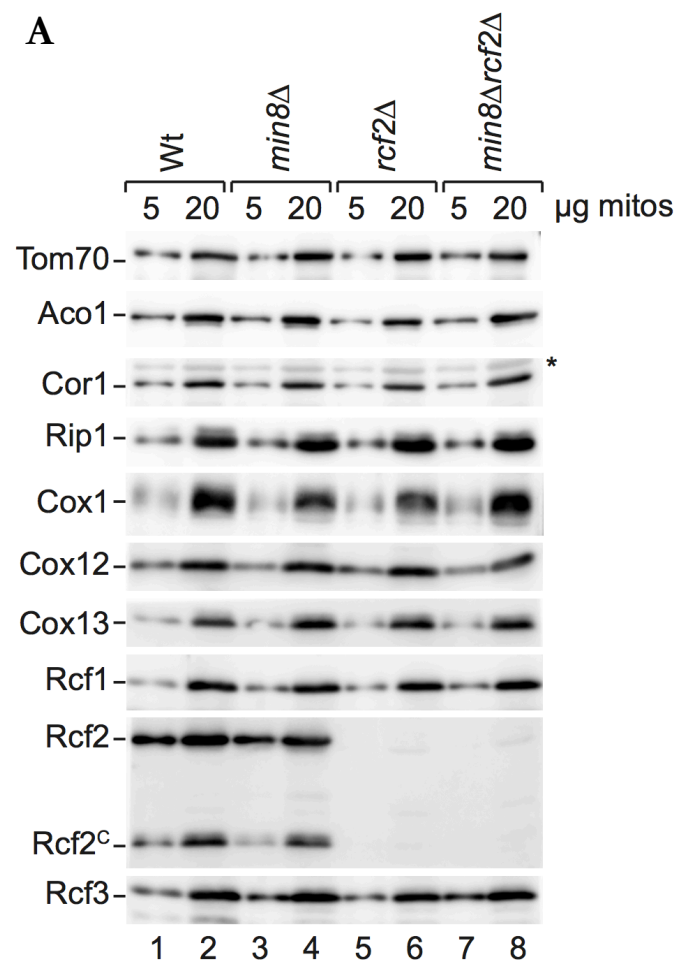

B
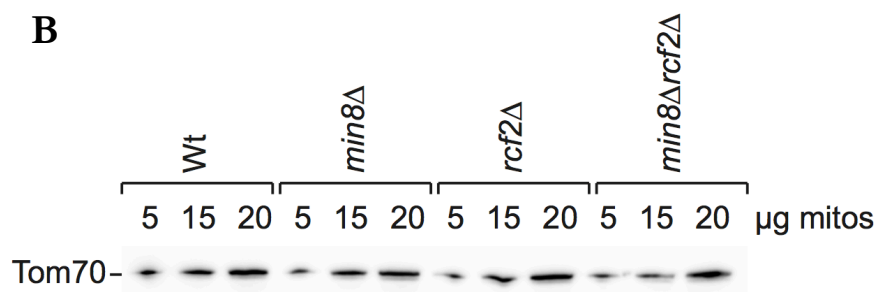

Cox $5 \mathrm{~b}$

$\begin{array}{llllllllllll}1 & 2 & 3 & 4 & 5 & 6 & 7 & 8 & 9 & 10 & 11 & 12\end{array}$

\section{C}

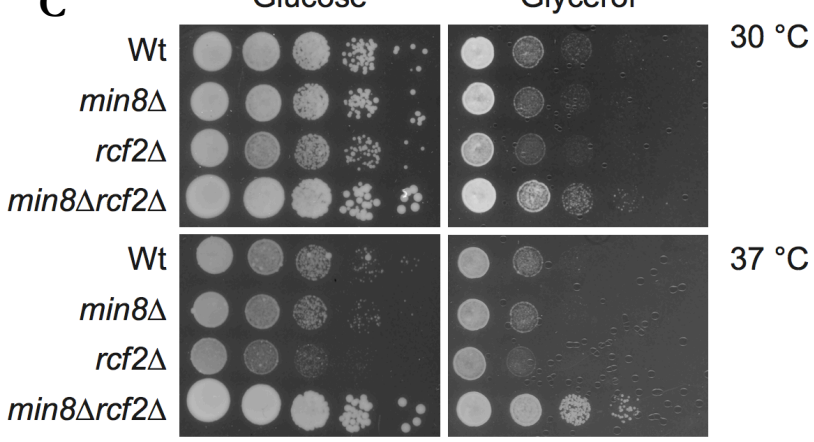

Figure 3-11: Min8 is not essential for respiration.

A) Isolated mitochondria of wild-type (Wt), $\min 8 \Delta, r c 2 \Delta, \min 8 \Delta r c f 2 \Delta$ were lysed in Laemmli-buffer and subjected to SDS-PAGE (10-16\%) and western blotting. Tom70 and Aco1 served as loading controls. Cor1 and Rip1 represent complex III, Cox1, Cox12 and Cox13 complex IV. The asterisk marks unspecific cross-reactions of the Cor1 antibody. B) As in A) but mitochondria are applied to urea SDS-PAGE (17.5\%) and western blotting. C) Wild-type, $\min 8 \Delta, r c f \Delta, \min 8 \Delta r c f 2 \Delta$ cells are spotted on plates in a serial dilution, containing glucose or glycerol media, and grown at $30{ }^{\circ} \mathrm{C}$ and $37^{\circ} \mathrm{C}$.

For specifying the role of Min8 and its position at the cytochrome $c$ oxidase, different $\mathrm{BN}$ analyses were carried out. To ensure that no complex IV constituent was missed in the prior steady state experiment, the first assessment was performed with DDM solubilized mitochondria. Assembled supercomplexes are resolved into complex IV plus the complex III 
dimer and the overall amount of complexes can be estimated. While complex IV can be detected as two distinct forms, $\mathrm{IV}^{*}$ and $\mathrm{IV}$, Cox13 is only part of the slower migrating complex $\mathrm{IV}^{*}$ (Figure 3-12A). This occurrence was discovered in previous studies and suggested complex IV* additionally containing Cox13, Rcf1 and Rcf2 (Vukotic et al., 2012). In both complex IV detections, the level of complex IV forms did not change significantly. Similar results were obtained when the respective mitochondria were analyzed after digitonin solubilization (Figure 3-12B). Although a slight tendency of $\mathrm{III}_{2} \mathrm{IV}_{2}$-reduction was detected in the particular min8 $\Delta$ strains (Figure 3-12B, lanes 2, 4, 6, 8, 10 and 12), it could not be proven as significant.

A

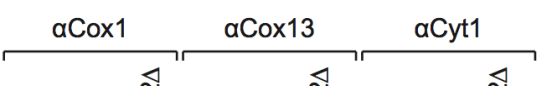

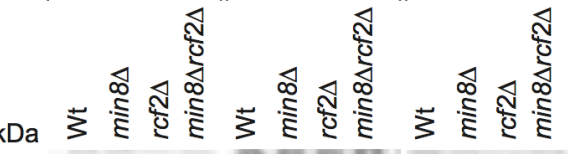

$880-$

$250-$

132

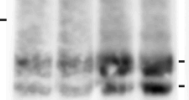

C

$\begin{array}{lllllllllllll}1 & 2 & 3 & 4 & 5 & 6 & 7 & 8 & 9 & 10 & 11 & 12\end{array}$

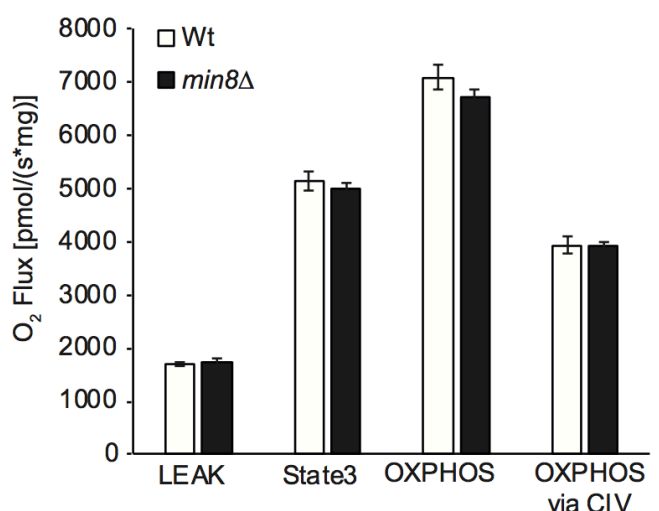

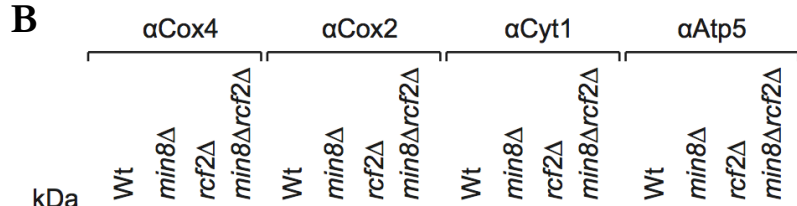

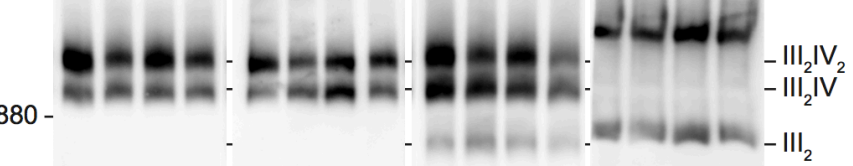

$440-$

$250-$

$132-$

$\begin{array}{llllllllllllllll}1 & 2 & 3 & 4 & 5 & 6 & 7 & 8 & 9 & 10 & 11 & 12 & 13 & 14 & 15 & 16\end{array}$

Figure 3-12: $M I N 8$ deletion does not affect respiration capacity.

A) Isolated wild-type, $\min 8 \Delta, \operatorname{rcf} 2 \Delta, \min 8 \Delta \operatorname{rcf} 2 \Delta$ mitochondria are solubilized with $0.6 \%$ DDM buffer and used for BlueNative (BN)-PAGE (6-10\%). Complex IV is detected via Cox1 and Cox13, and complex III via Cyt1. B) As in A) but mitochondria were solubilized with $1 \%$ digitonin buffer and subjected to BN-PAGE (4-10\%). Cox4 and Cox1 represent complex IV and Atp5 complex V. C) Oxygen consumption measurement of isolated wild-type and min $8 \Delta$ mitochondria with the Oxygraph-2k ( $\mathrm{n}=3$ ). LEAK: non-phosphorylating state; State 3: upon addition of saturating ADP; OXPHOS: maximum respiration under coupled conditions; OXPHOS via CIV: complex IV activity in parallel to complex III inhibition. Error bars indicate mean \pm SEM. 
Expecting a more sensitive outcome in a respirometry approach, min $8 \Delta$ mitochondria were subjected to the oxygen consumption measurements with the Oxygraph-2k, in comparison to wild-type mitochondria. The oxygen consumption was determined in the different states LEAK, state 3, OXPHOS and OXPHOS via complex IV (Figure 3-12C). The terminology is guided by the Oxygraph-2k manufacturer's specification (Gnaiger 2020).

First, mitochondria are applied to the oxygen chamber containing the respiration medium with appropriate conditions to maintain the mitochondria intact. Mutant and wild-type mitochondria are always measured next to each other to keep the conditions stable for comparison. Pyruvate and Malate are added to allow LEAK respiration - oxygen consumption without added phosphorylating substrates. By this, high proton leakage would be visible since protons cannot escape into the mitochondrial matrix along the membrane potential via complex V. State 3 allows ATPase performance and is triggered by adding ADP to saturating conditions. OXPHOS represents the maximum state of respiration under coupled conditions. For this, succinate is supplemented. Complex IV activity, essentially OXPHOS via complex IV, can be measured in the end by inhibiting complex III with antimycin A and shuttling electrons specifically to complex IV with TMPD and ascorbate. Ascorbate regenerates TMPD, but both undergo autoxidation which is appreciated by sodium azide addition and the residual oxygen consumption is subtracted in the end. All four measured states did not display any difference between min $8 \Delta$ and wild-type mitochondria.

In the end, these basic characterizing experiments could not assign a specific role for Min8 as complex IV interactor: it is not essential for respiration, nor does it influence the arrangement or the capacity of the respiratory chain. However, the significant interaction with Cox12 detected by chemical crosslinking indicated a close interaction at the periphery of complex IV. Consequently, we were wondering if Min8 even plays a role in its biogenesis.

\subsubsection{Min8 affects Cox12 assembly into cytochrome c oxidase}

Investigating the import of radioactively labeled Cox12 and Cox13 into energized mitochondria, we were aiming for evaluating the influence of Min8 on late-stage complex IV assembly in general. By choosing appropriate timepoints and BN analysis, the assembled protein is detectable in the ultimate complex and/or maturating stages of the complex. Mitochondria with disrupted membrane potential $(-\Delta \psi)$ represent the negative control for Cox13 import. Cox13 is a TIM23 substrate and its import is membrane potential dependent, while Cox12 is an IMS protein and a MIA substrate (see section 1.3.2). Thus, Cox12 import is not membrane potential 
dependent but relies on cysteine-cysteine interactions. These can be disrupted with the cysteine alkylating reagent iodoacetamide (IAA) and serves as a negative control. Import is stopped after defined time points by disrupting the proton motive force and with a mixture of antimycin A, valinomycin, oligomycin (AVO) and the redox-activity of Mia40 with IAA, respectively.

We compared wild-type and min $8 \Delta$ mitochondria to each other after import of Cox13 and Cox12 and solubilization with $0.6 \%$ DDM (Figure 3-13A). As before, cytochrome $c$ oxidase is detectable as a monomer which serves quantification purposes. Additionally, cox $4 \Delta$ mitochondria were used to show significance for complex IV assembly, since it lacks mature cytochrome $c$ oxidase.

A

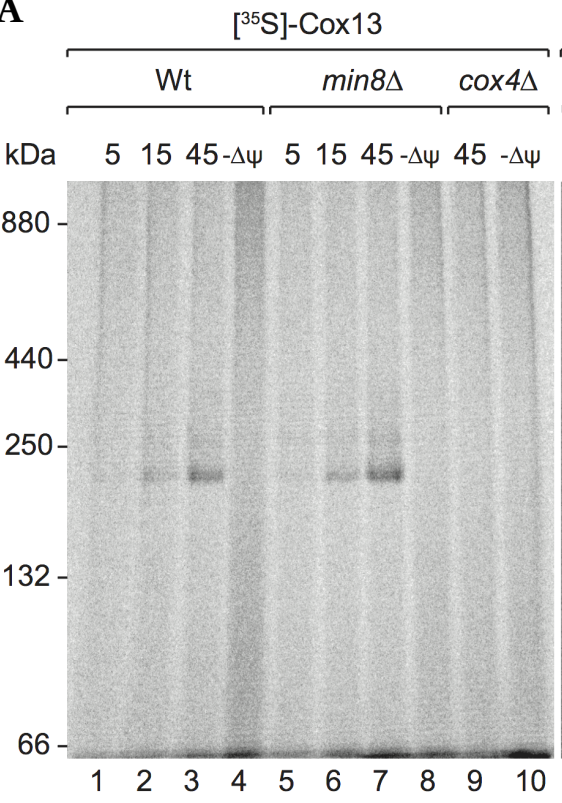

$\left[{ }^{35} \mathrm{~S}\right]-\operatorname{Cox} 12$

\begin{tabular}{|c|c|}
\hline Wt & $\min 8 \Delta$ \\
\hline
\end{tabular}

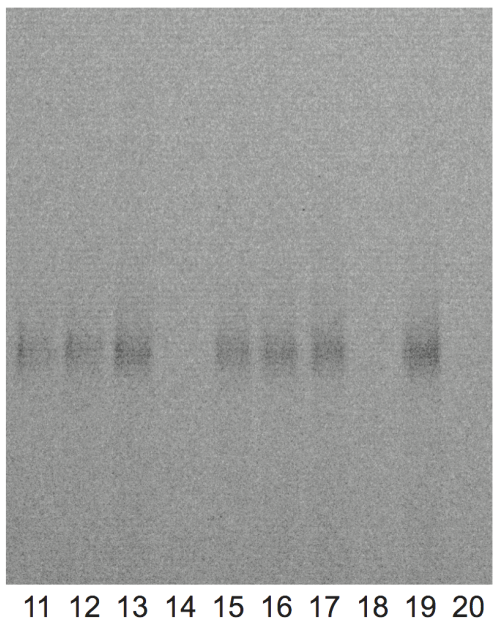

B

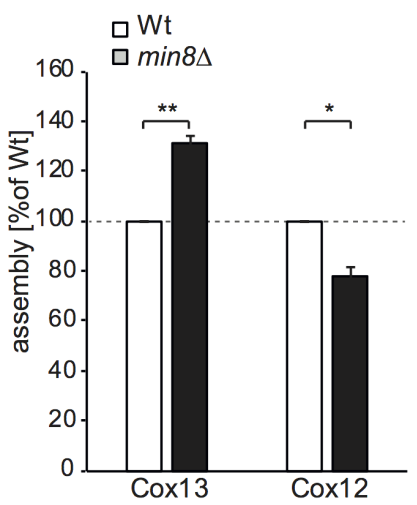

Figure 3-13: Min8 influences late stage assembly of cytochrome $c$ oxidase.

A) ${ }^{35}$ S-labeled Cox 13 and Cox12 were imported into energized wild-type (Wt), min $8 \Delta$, $\operatorname{cox} 4 \Delta$ mitochondria. Negative controls were treated before with an antimycin A, valinomycin, oligomycin (AVO) mix, to disrupt membrane potential (- $\Delta \psi$ ), and IAA to inhibit cysteine-cysteine interactions in case of Cox12. Imports were stopped with AVO and IAA, respectively, after indicated timepoints. Mitochondria were solubilized with $0.6 \%$ DDM buffer and subjected to BN-PAGE (6-10\%). Gels were dried, exposed to phosphoscreen and analyzed via autoradiogram. B) Quantification of 45 min timepoint of $n=3$ experiments from A) in ImageJ with background subtraction (Schindelin et al., 2012). Normalization was performed to wild-type signals, error bars indicate means \pm SEM. Statistical significance was determined with a two-tailed unpaired t-test $\left({ }^{*} p<0.05,{ }^{* *} p<0.005\right)$.

Surprisingly, we could not detect Cox12 assembling into mature complex IV but in a slightly faster migrating complex which was not seen before. Additionally, Cox12 assembly into this complex seems to occur independent of mature complex IV. The cox $4 \Delta$ mutant displays an accumulated complex with even higher intensity than the other two strains after 45 min (Figure 3-13A, lane 19). Comparing min $8 \Delta$ against wild-type, Cox13 assembly appears to be specifically more efficient in the mutant, while Cox12 assembly is hindered (Figure 3-13B). 
Although Min8 as a putative novel complex IV subunit did not significantly alter the considered protein environment of the respiratory supercomplexes under tested conditions, we could assign a role for late-stage complex IV assembly - by facilitating Cox12 assembly into a presumed complex IV intermediate on the one side and regulating Cox13 assembly on the other.

\subsection{Functionality assessment of the Rcf-domains}

Since we could now identify specific interaction sites of Rcf2 and Rcf3 at complex IV and even broaden the spectrum of complex IV associated proteins, an analysis of the respective transmembrane domains of Rcf-proteins was the next step. By this we were aiming for valuable information to define the so far indistinct functions of the Rcf-proteins. Evaluating the transmembrane features of Rcf-proteins, it was remarkable that the Rcf2 sequence comprises homologies to both, Rcf3 and Rcf1 (section 3.1.1, Figure 3-1A) (Römpler et al., 2016). In addition, an internal processing event of Rcf2 with unknown purpose was described previously. The C-terminus of Rcf2 harbors a conserved HIG1 domain, homologous to Rcf1, while the $\mathrm{N}$-terminus shares a homologous sequence with Rcf3 (Römpler et al., 2016). Following this, we were wondering if the respective domains would complement one another or if they display distinct functions and we could identify a purpose for Rcf2 processing.

Conclusively, we constructed fusion proteins of Rcf3 and Rcf1 and swap proteins with Rcf2. To this end, respective domains of Rcf2 were exchanged with the corresponding homologous protein partner. This resulted in the artificial constructs Rcf3-Rcf1, Rcf2 ${ }^{\mathrm{N}}-\operatorname{Rcf1}$ and $\operatorname{Rcf3}-\mathrm{Rcf} 2^{\mathrm{C}}$.

\subsubsection{Mimicking Rcf2 with fusion proteins}

For transforming the fusion proteins into the cells, the particular coding sequences for the protein regions were fused and cloned into a single copy plasmid for yeast expression. The proteins were expressed under the respective endogenous promotor of the $\mathrm{N}$-terminal protein, while being aware that this ignores possible differences in protein levels. Regarding the conserved HIG1 domain (aa89-180) and keeping it intact, we decided the Rcf2 sections to be $\operatorname{Rcf2}^{\mathrm{N}}$ (aa1-83) and Rcf2 ${ }^{\mathrm{C}}$ (aa84-224) (Figure 3-14A).

The first assessment showed that all three artificial proteins are expressed in yeast cells and successfully imported into mitochondria. The proteins can be detected with the respective antibodies, yet the signals indicate that their expression level differs (Figure 3-14B). Comparing the signals to the respective endogenous protein, the difference is not as pronounced, except 
for Rcf3-Rcf2 ${ }^{\mathrm{C}}$. The protein is detected with the antibody against Rcf3 and binding might be disturbed due to the additional C-terminus containing Rcf2. Detection of this protein with the Rcf2 antibody is possible in general, but the signals overlap in the chosen gel system.

Nonetheless, we proceeded with those constructs, but it was important to consider this possible difference for interpretation. When the constructs were first expressed in wild-type cells, steady state analysis did not show significant differences in the tested proteins related to respiration. Furthermore, expressing additional parts of the proteins does not interfere with the endogenous protein expression and the Rcf-protein levels remain stable.

A
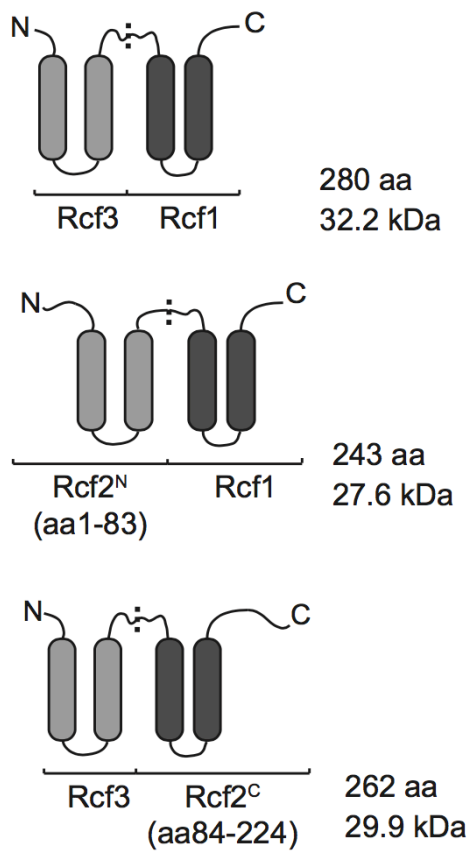

B

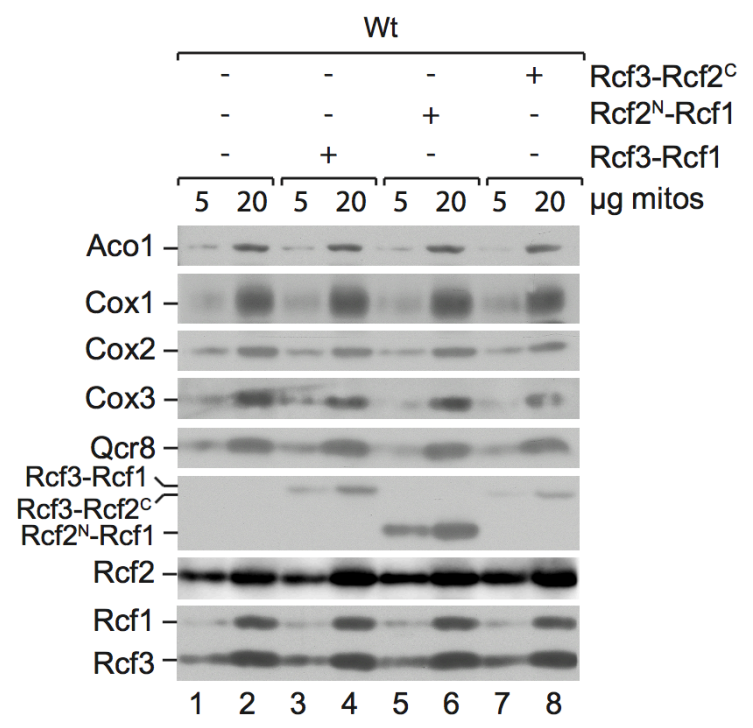

Figure 3-14: Expressing fusion proteins in yeast.

A) Model representing the constructed fusion proteins Rcf3-Rcf1, Rcf2 ${ }^{\mathrm{N}}-\mathrm{Rcf1}$, Rcf3-Rcf2C. Light grey boxes indicate putative transmembrane spans representing/homologous to the $\mathrm{N}$-terminus of Rcf2, dark grey boxes indicate transmembrane spans representing/homologous to the C-terminus of Rcf2. B) Isolated mitochondria of wild-type (Wt) cells expressing Rcf3-Rcf1, Rcf2N-Rcf1 or Rcf3-Rcf2C, where indicated, were lysed in Laemmli-buffer, subjected to SDS-PAGE (10-16\%) and western blotting. Fusion proteins were detected via mixture of Rcf1 and Rcf3 antibodies.

\subsubsection{Rcf3-Rcf1, Rcf2 ${ }^{\mathrm{N}}-\mathrm{Rcf} 1$ and $R c f 3-R c f 2^{\mathrm{C}}$ are functional mitochondrial proteins}

In order to examine the functionality, we introduced the different fusion proteins into the mutants $r f f 1 \Delta$ and $r r f 2 \Delta r f 3 \Delta$ and evaluated the respective protein complementation in a growth drop test. The mutants alone are not able to respire on non-fermentable media and display a 
heavy growth phenotype (section 3.1.2). Functional proteins would overcome the respiration disability and the corresponding strain regains growth on non-fermentable media.

The experiment showed that Rcf3-Rcf1, Rcf2 ${ }^{\mathrm{N}}-\operatorname{Rcf1}$ and $\mathrm{Rcf3}-\mathrm{Rcf} 2^{\mathrm{C}}$ were able to compensate the $\operatorname{rcf} 2 \Delta r f 3 \Delta$ growth phenotype (Figure 3-15). Cells expressing the artificial proteins recover almost wild-type like growth. This was surprising; especially that both proteins containing parts of Rcf2 could either function as Rcf2 or Rcf3. However, if Rcf3-Rcf2 ${ }^{C}$ is behaving like Rcf2 or Rcf3 cannot be distinguished in this experimental setup. It seemed intuitive, that $r f f 1 \Delta$ cells expressing Rcf2 ${ }^{\mathrm{N}}$-Rcf1 or Rcf3-Rcf1 could overcome the mutant's growth phenotype as well. Although not achieving completely wild-type like growth ability, it was a remarkable improvement.
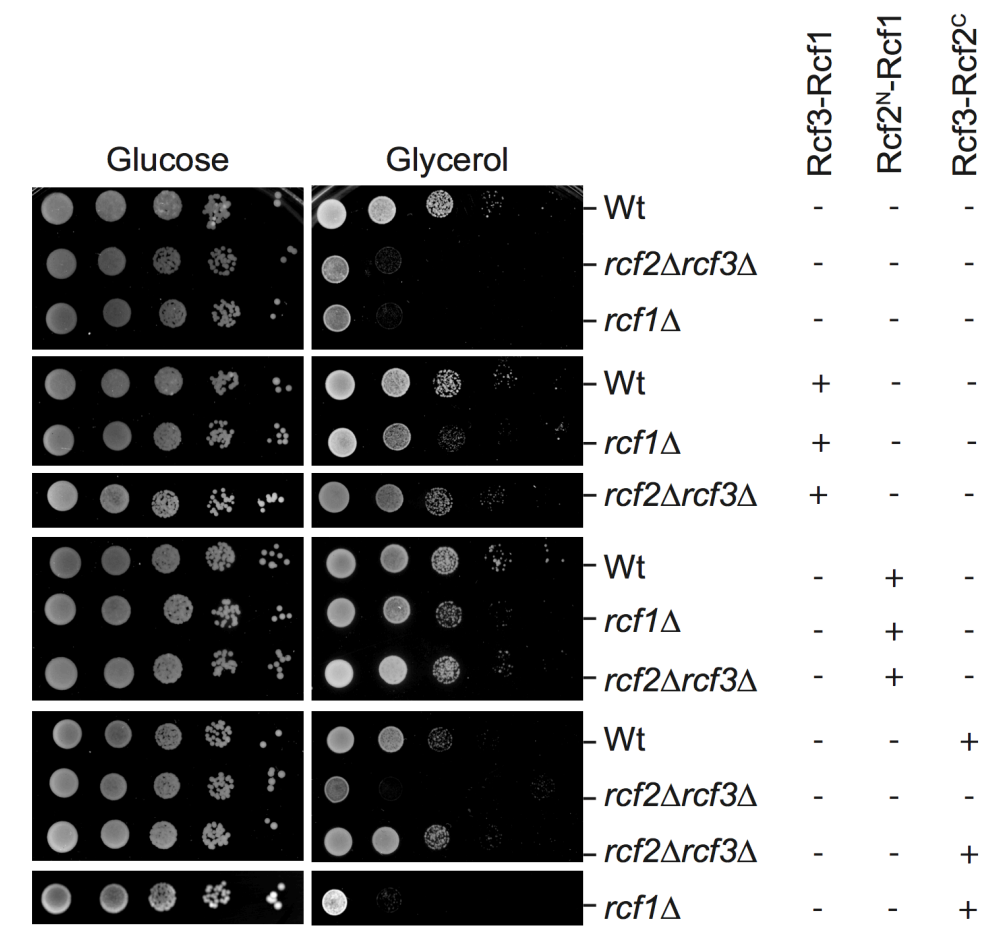

Figure 3-15: Fusion proteins can substitute for Rcf-proteins.

Cells of the indicated strains were spotted on glucose and glycerol plates in a serial dilution and grown for several days at $30^{\circ} \mathrm{C}$. As controls, wild-type cells and at least one mutant $(r f 1 \Delta, r c 2 \Delta r c \beta 3 \Delta)$ expressing the empty plasmid were spotted next to the investigated strains.

At the same time, Rcf3-Rcf2 ${ }^{\mathrm{C}}$ could not restore respiration in $r c f 1 \Delta$. This was partly expected, since Rcf2 itself cannot substitute for Rcf1 function, as apparent in $r f 1 \Delta$. For that reason, the $r c f 1 \Delta$ strain expressing Rcf3-Rcf2 ${ }^{\mathrm{C}}$ was not taken along for the following experiments.

In order to examine if the proteins can influence protein levels in the mutants, a rough steady state analysis was carried out with the respective isolated mitochondria. Considering the low impact of RCF deletions in terms of protein levels, only the core complex IV subunits (Cox1, 
Cox2, Cox3) plus Qcr8 as a complex III constituent were probed additionally to the Rcf-proteins (Figure 3-16). When expressed in $r f f 1 \Delta$, both Rcf2 ${ }^{\mathrm{N}}$-Rcf1 and Rcf3-Rcf1 were able to restore the subtle Cox3 decrease (Figure 3-16A), which was determined before in section 3.1.2 (Figure 3-3A). Again, the expression of the different fusion proteins did not alter Rcf-protein expression (compare section 3.4.1).
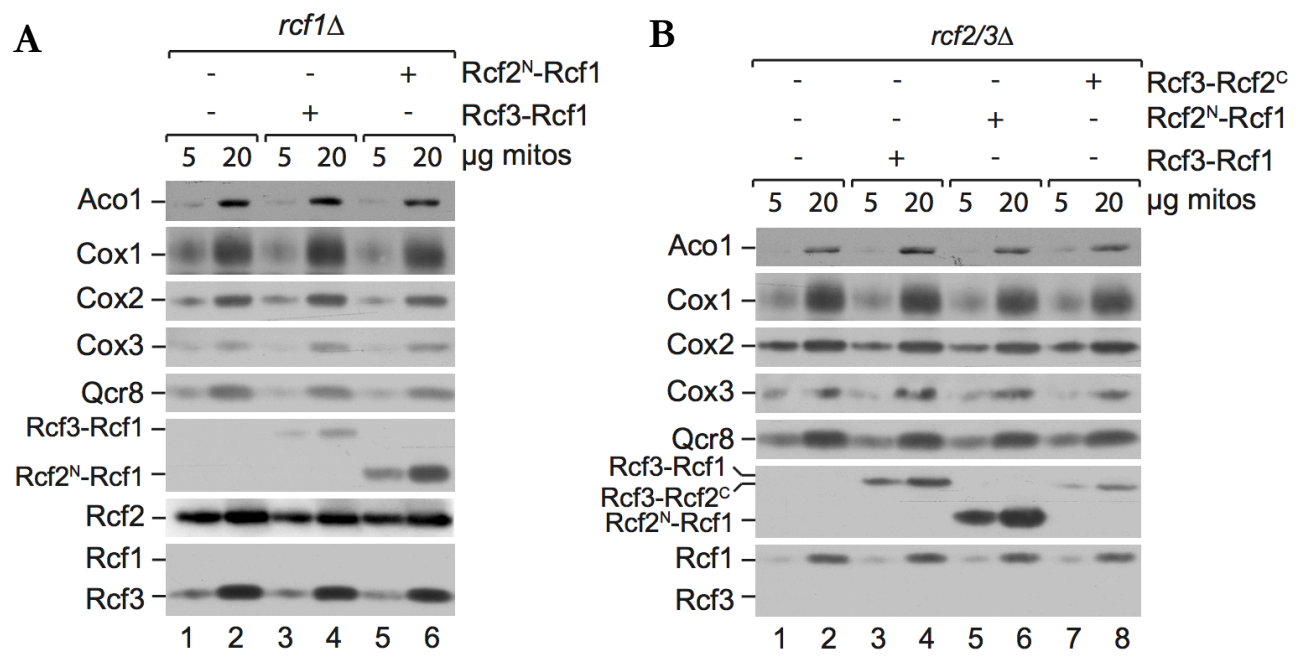

Figure 3-16: Fusion proteins do not influence protein levels.

A) Isolated mitochondria of $r f 1 \Delta$ cells expressing Rcf3-Rcf1 or Rcf2N-Rcf1, where indicated, were lysed in Laemmli-buffer, subjected to SDS-PAGE (10-16\%) and western blotting. Fusion proteins were detected via mixture of Rcf1 and Rcf3 antibodies. B) As in A) with isolated mitochondria of $r f 2 \Delta r c 3 \Delta$ cells expressing Rcf3-Rcf1, Rcf2N-Rcf1 or Rcf3-Rcf2C, where indicated.

After assessing general functionality, we were further interested if the fusion constructs would behave Rcf-protein like or even Rcf2 like in regard of supercomplex association. This was determined via degenerating $2^{\text {nd }}$ dimension SDS analysis after BN-PAGE of isolated mitochondria from wild-type cells expressing the respective fusion protein (Figure 3-17). By this, the distinct complexes are first resolved in their native conformation and subsequently the participating proteins are separated upon SDS lysis.

The complex III subunit Qcr8 served as a control for respiratory supercomplexes. While Rcf1 and Rcf2 clearly co-migrate with the $\mathrm{III}_{2} \mathrm{IV}_{(1-2)}$ complexes Rcf3 displays only weak association with the supercomplexes (Figure 3-17). The majority of Rcf-proteins however, migrates within smaller molecular weight complexes. All three fusion proteins in turn, could be determined as colocalizing at the complexes $\mathrm{III}_{2} \mathrm{IV}_{(1-2)}$, although to a different extent. While Rcf3-Rcf1 and Rcf3-Rcf2 ${ }^{\mathrm{C}}$ could be detected in amounts comparable as Rcf2 and Rcf1 at $\mathrm{III}_{2} \mathrm{IV}_{(1-2)}$, Rcf2 ${ }^{\mathrm{N}}-\mathrm{Rcf}_{1}$ harboring complexes seem to be smaller and faster migrating. 
In conclusion, all constructs Rcf3-Rcf1, Rcf2 ${ }^{\mathrm{N}}$-Rcf1 and Rcf3-Rcf2 ${ }^{\mathrm{C}}$ were conceived as functional mitochondrial proteins. Although expressed under different promotors, they could successfully restore the detected growth phenotypes for $\operatorname{rcf} 1 \Delta$ and $\operatorname{rcf} 2 \Delta \operatorname{rcf} 3 \Delta$ and did not alter any protein levels in a harmful way. Additionally, it was shown that they function Rcf-protein like by residing at the respiratory supercomplexes.
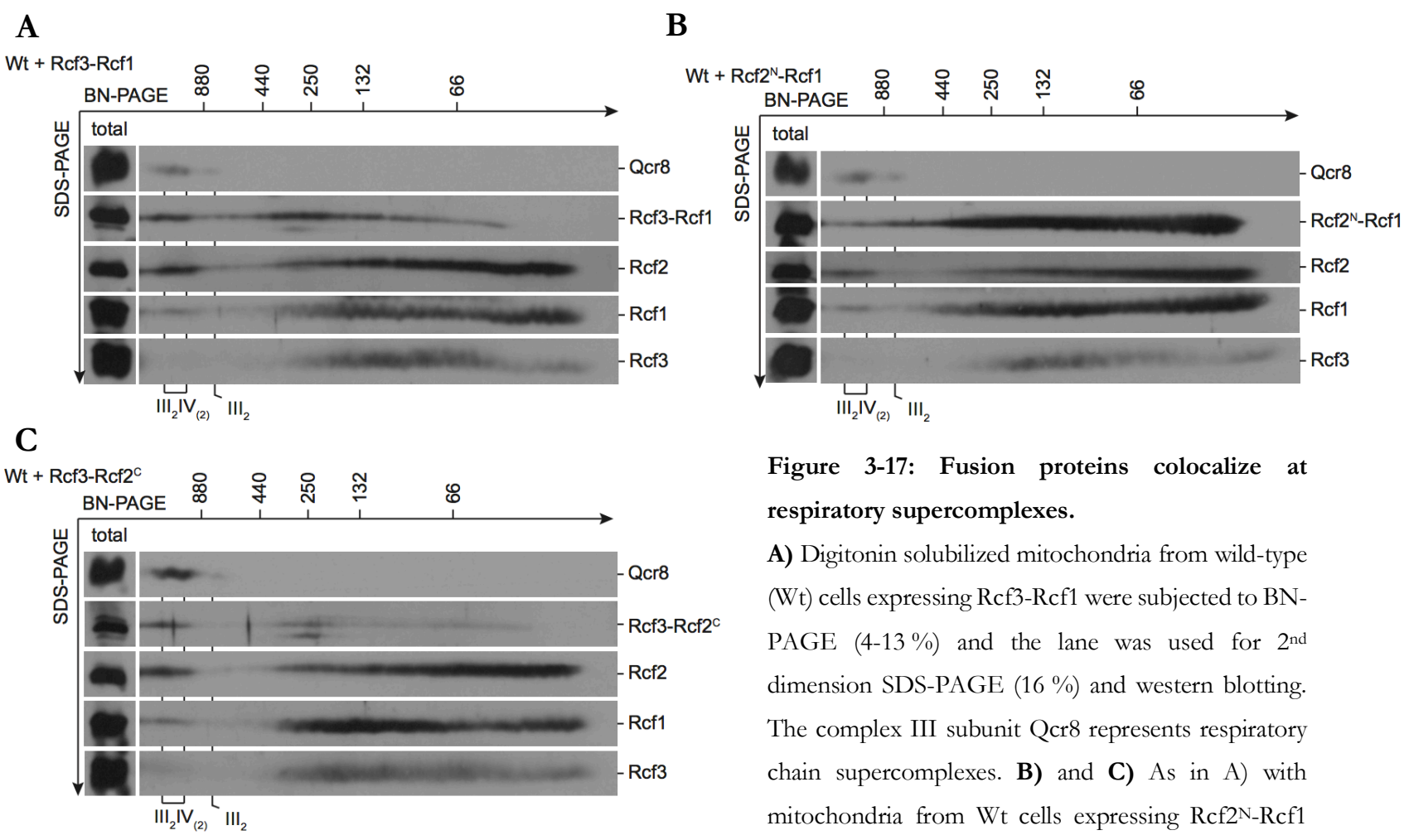

Figure 3-17: Fusion proteins colocalize at respiratory supercomplexes.

A) Digitonin solubilized mitochondria from wild-type (Wt) cells expressing Rcf3-Rcf1 were subjected to BNPAGE (4-13\%) and the lane was used for $2^{\text {nd }}$ dimension SDS-PAGE (16\%) and western blotting. The complex III subunit Qcr8 represents respiratory chain supercomplexes. B) and C) As in A) with mitochondria from Wt cells expressing Rcf2 ${ }^{\mathrm{N}}-\mathrm{Rcf} 1$ and Rcf3-Rcf2C, respectively.

\subsubsection{Rcf2 $^{\mathrm{N}}$-Rcf1 and Rcf3-Rcf1 facilitate supercomplex assembly}

Besides the systemic phenotype of $r f 1 \Delta$ represented in failing respiration, the RCF1 deletion furthermore causes a rearrangement of the respiratory chain, described above (section 3.1.2). In order to determine if the fusion proteins with Rcf1 can rescue this phenotype and function as such, we conducted $\mathrm{BN}$ analysis with digitonin solubilized mitochondria.

In fact, Rcf3-Rcf1 and Rcf2 ${ }^{\mathrm{N}}$-Rcf1 were both able to restore the respirasome assembly (Figure 3-18). Complex III was detected with Rip1 and complex IV with Cox4 antibody. Both obtained native complex formation demonstrate the clear phenotype of $r f 1 \Delta$ ((Figure 3-18A+B, lanes 9 and 15) and an almost wild-type like organization when Rcf3-Rcf1 or Rcf2 ${ }^{\mathrm{N}}$-Rcf1 (Figure 3-18, lanes 10 and 16) were expressed additionally. 
A

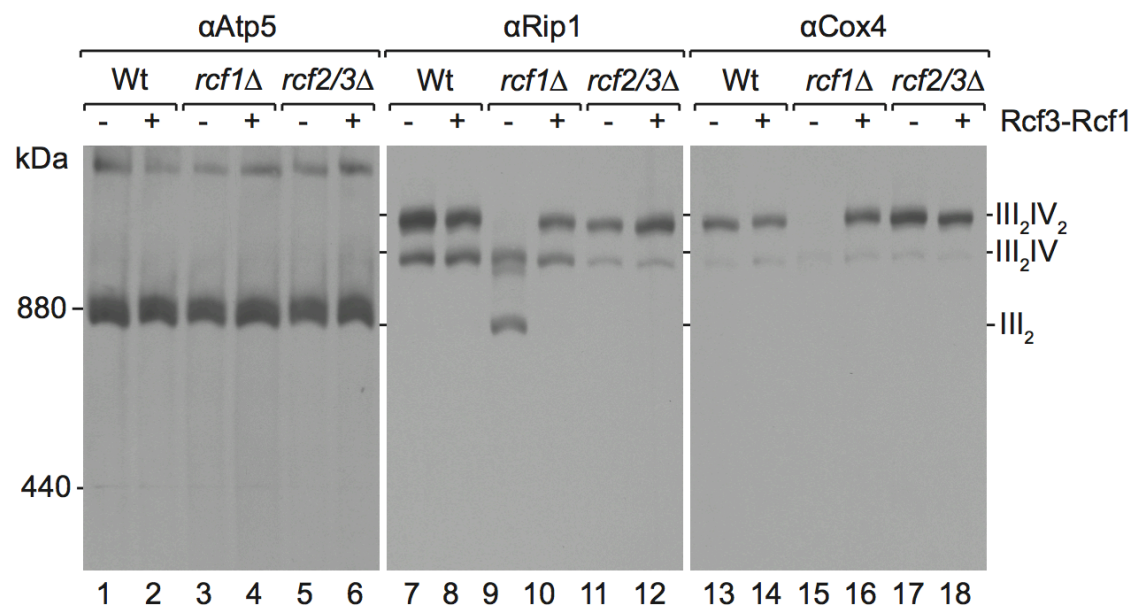

B

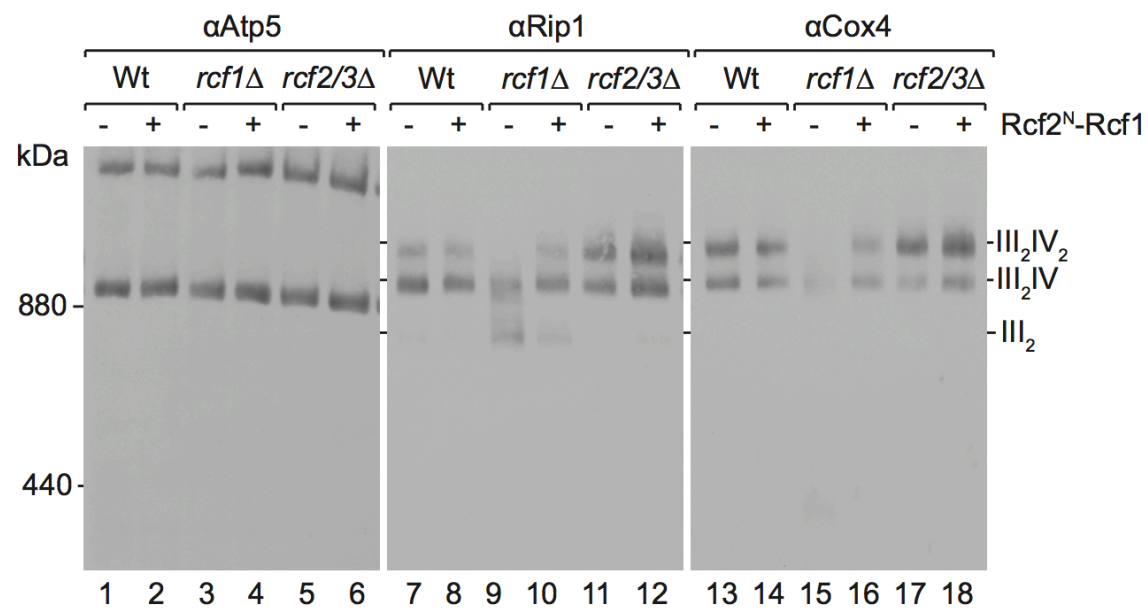

Figure 3-18: Expression of Rcf3-Rcf1 and Rcf2 ${ }^{\mathrm{N}}-\mathrm{Rcf1}$ rescues $r c f 1 \Delta$ phenotype in supercomplex arrangement.

A) and B) Digitonin solubilized mitochondria from wild-type (Wt), $r f 1 \Delta$ and $r f 2 \Delta r c f 3$ cells expressing Rcf3-Rcf1 and Rcf2N-Rcf1, where indicated, were applied to BN-PAGE (4-10\%) and western blotting. Atp5 as complex V subunit serves as a loading control, complex III is detected via Rip1 and complex IV via Cox4.

Rcf2 ${ }^{\mathrm{N}}$-Rcf1, however, could not overcome the disbalanced arrangement completely, in contrast to Rcf3-Rcf1. This indicates that both proteins can fulfill a role as Rcf1, but with the Rcf2 ${ }^{\mathrm{N}}$ fragment attached, the capacity is less pronounced. Wild-type and $\operatorname{rcf} 2 \Delta \operatorname{rcf} 3 \Delta$ do not show any significant alteration in supercomplexes when the artificial proteins are present.

\subsubsection{Rcf3-Rcf1 and Rcf2 ${ }^{\mathrm{N}}$-Rcf1 support Cox13 assembly}

Additionally to the reported support of Rcf1 during supercomplex assembly, it was previously shown that Rcf1 is playing a role in Cox13 assembly (Vukotic et al., 2012). Another interesting finding in that study is that distinct subsets of cytochrome $c$ oxidase exist - with and without Cox13. These can easily be detected by DDM solubilization and thereby disruption of the respiratory supercomplexes on BN-PAGE. To further review the effects of the fusion proteins, DDM solubilized mitochondria from the different wild-type and mutant cells expressing the 
constructs were used for BN-PAGE analysis. Especially in regard to the observed phenotype of $r f 1 \Delta$, we were questioning if Cox13 assembly can be modulated.

As expected, almost no complex IV* is detectable in $r f 1 \Delta$ and Cox13 assembly is heavily impaired (Figure 3-19, lanes 5 and 16). At the same time, we could demonstrate that Rcf3-Rcf1 can restore Cox13 assembly to almost wild-type levels. Rcf2 ${ }^{\mathrm{N}}$-Rcf1was able to rescue the phenotype to some extent, however, Cox13 incorporating complex IV* appeared still at a decreased level. Interestingly, we could observe a slight decrease in complex IV* level in the wild-type with $\operatorname{Rcf} 2^{\mathrm{N}}$-Rcf1 being present, as well. The double mutant, expressing either of the fusion proteins, did not show any difference in complex CIV* levels.

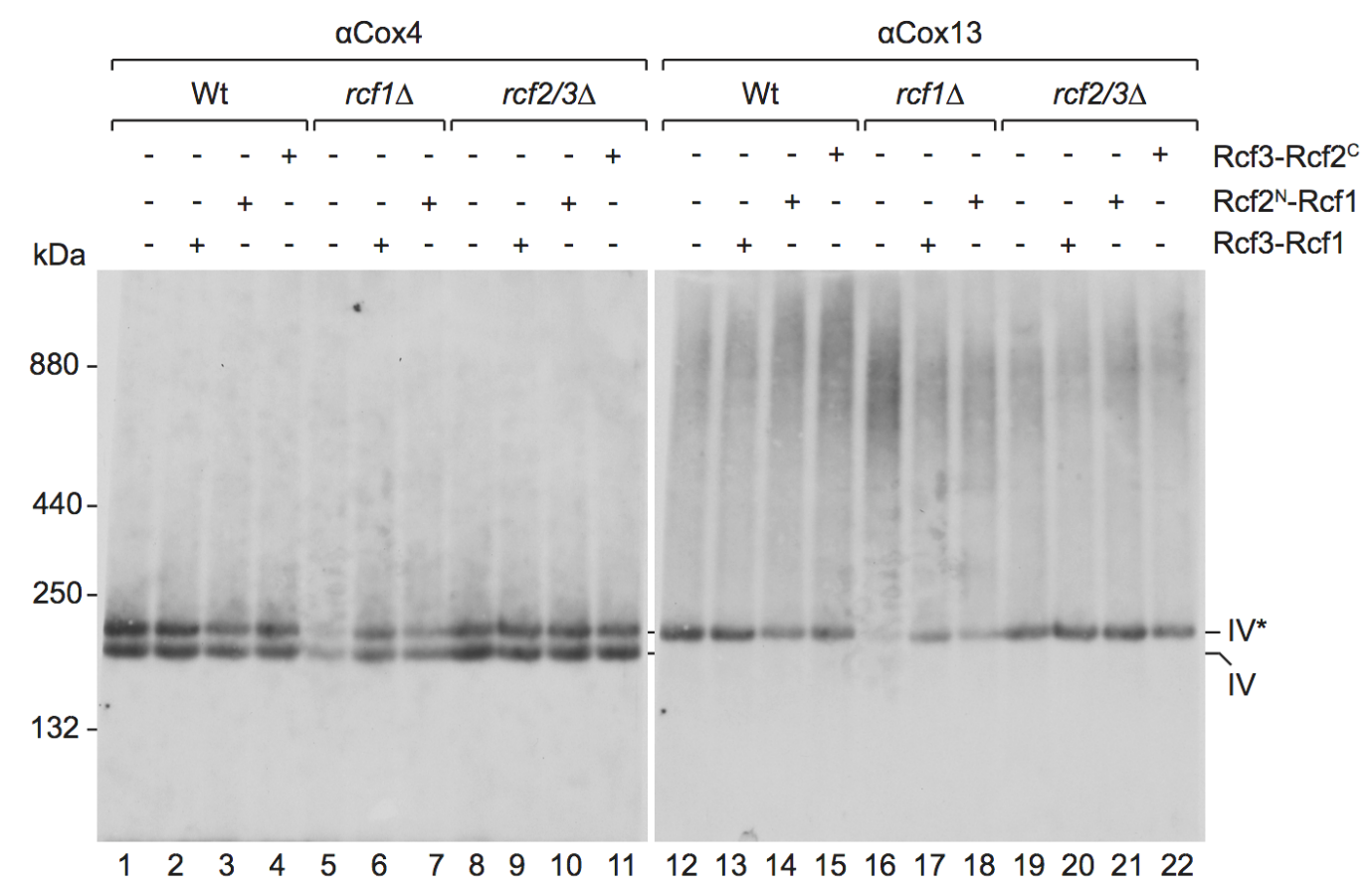

Figure 3-19: Rcf3-Rcf1 and Rcf2N-Rcf1 compensate Cox13 assembly phenotype in $r c f 1 \Delta$.

$\operatorname{DDM}(0.6 \%)$ solubilized mitochondria from wild-type (Wt), $r c f 1 \Delta$ and $r f 2 \Delta r f 3 \Delta$ cells expressing Rcf3-Rcf1, Rcf2 $2_{-R}$ cf1 and Rcf3-Rcf2C, where indicated, were applied to BN-PAGE (6-10\%) and western blotting.

Consequently, the fusion proteins containing Rcf1 can support Cox13 assembly in a RCF1 deletion situation. At the same time, the N-terminal transmembrane spans of Rcf2 fused to Rcf1 seem to interfere with the function of Rcf1 in regard to Cox13 assembly.

\subsubsection{Fusion proteins act flexible upon respiration}

The expression of the fusion proteins Rcf3-Rcf1 and Rcf2 ${ }^{\mathrm{N}}$-Rcf1 could compensate not only the phenotypes on the protein level but also within the in vivo growth test on non-fermentable media (section 3.4.2). With the experiments of the isolated mitochondria in mind, we were questioning how the fusion constructs manage the situation with none of the Rcf-proteins being 
present. Thus, the test was repeated with $r c f 1 \Delta r f 2 \Delta r f 3 \Delta$ cells expressing the fusion proteins (Figure 3-20). The construct Rcf3-Rcf2 ${ }^{\mathrm{C}}$ was left out in this case, since it did not improve respiration on glycerol in $r c f 1 \Delta$, initially.

To provide evidence for specificity, Rcf3 and Rcf1 were expressed in the triple RCF mutant to exclude that already one of the proteins has an impact on respiratory function. Interestingly, both proteins Rcf3-Rcf1 and Rcf2 ${ }^{\mathrm{N}}$-Rcf1 were able to rescue the heavily affected $\operatorname{rcf} 1 \Delta \operatorname{rcf} 2 \Delta \operatorname{rcf} 3 \Delta$ in terms of growth on glycerol. Meanwhile, Rcf3 or Rcf1 alone failed to enhance respiration ability, when expressed in the triple mutant. This was expected, since $r f 2 \Delta r c f 3$ and $r c f 1 \Delta r c f 2 \Delta$ do show a similar disrupted function. The combined deletion of RCF1 and RCF2 corresponds to published results of Strogolova et al. (2012) and Dawitz et al. (2020). Both studies indicate that this impaired respiration ability is even more distinct than of $r c f 1 \Delta$ (Strogolova et al., 2012; Dawitz et al., 2020).

It could not be determined in this setup if the domains are able to operate within the same protein or from different proteins. However, the proteins could accomplish a function as Rcf2 or Rcf3 and Rcf1 at the same time. Conclusively, the proteins are capable of using the appropriate domains flexibly and adapt to the particular demands.

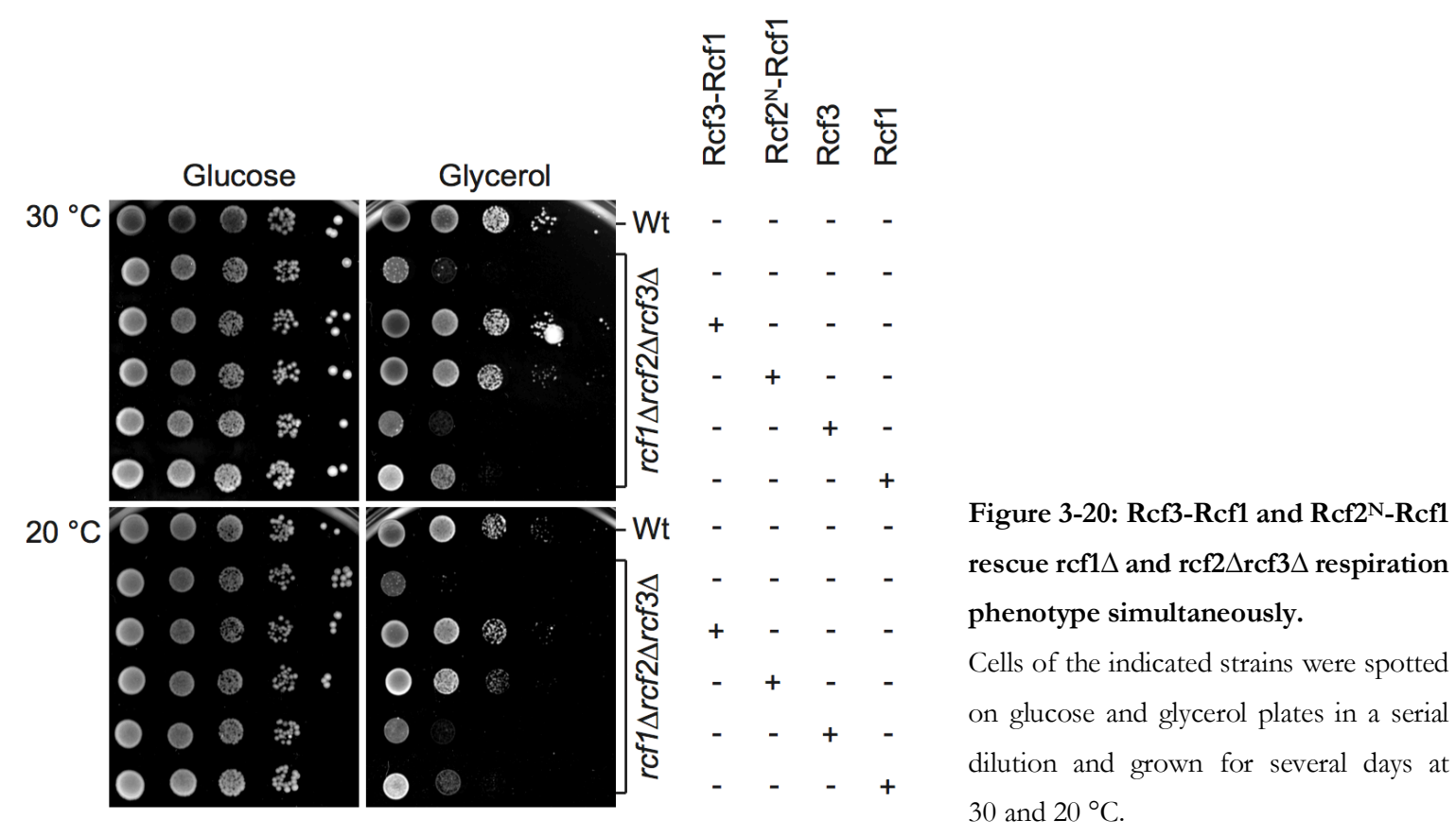




\subsubsection{Rcf2 fragments $\operatorname{Rcf} 2^{\mathrm{N}}$ and $\operatorname{Rcf} 2 \mathrm{C}$ alone are not functional}

As described before in section 3.4.2, the fusion constructs harboring parts of Rcf2 were able to compensate the respiration phenotype detected in $r f 2 \Delta r f 3 \Delta$. Since this was entirely unexpected, it was important for us to study the effects of Rcf2 fragments alone. We were questioning if both parts of Rcf2 alone would have the same ability. Thus, plasmids encoding for $\operatorname{Rcf2}^{\mathrm{N}}$ (aa1-83) and Rcf2 ${ }^{\mathrm{C}}$ (aa84-224) were transformed into $r f 2 \Delta r f 3 \Delta$ and into wild-type cells.

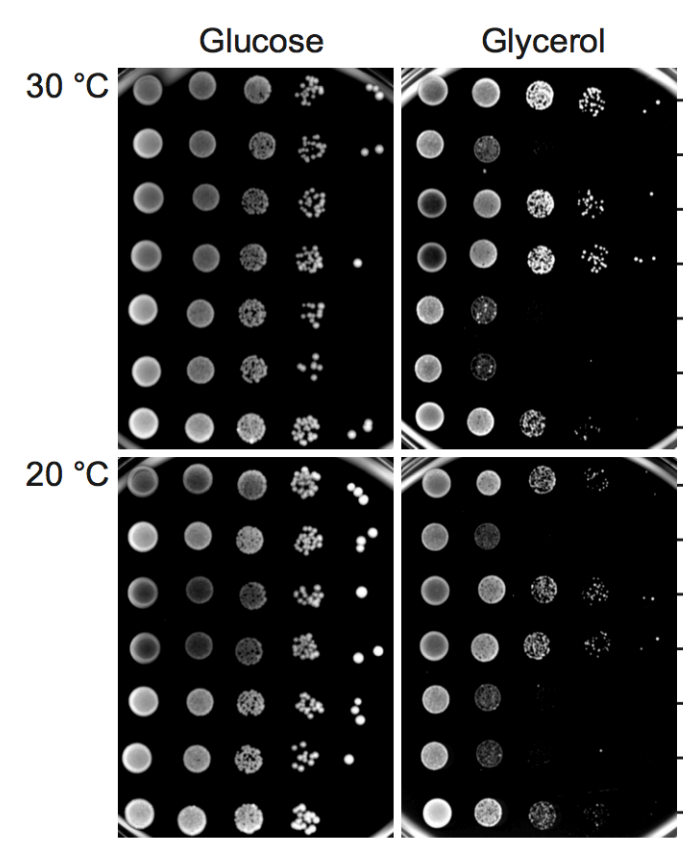

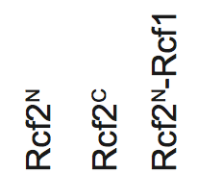
Wt $\mathrm{rcf} 2 / 3 \Delta$ Wt - Wt $\mathrm{rcf} 2 / 3 \Delta$ $\mathrm{rcf} 2 / 3 \Delta$ $\mathrm{rcf} 2 / 3 \Delta$ $\mathrm{Wt}$ $\operatorname{rcf} 2 / 3 \Delta$ Wt Wt $\mathrm{rcf} 2 / 3 \Delta$ rcf2/3 $\Delta$ $\mathrm{rcf} 2 / 3 \Delta$
Figure 3-21: $\operatorname{Rcf} 2^{\mathrm{N}}$ and $\operatorname{Rcf} 2^{\mathrm{C}}$ fragments alone are not functional. Cells of the indicated strains were spotted on glucose and glycerol plates in a serial dilution and grown for several days at 30 and $20^{\circ} \mathrm{C}$.

We subsequently performed a growth drop-test with the respective strains and the result proved that the rescue of respiration phenotype of $r f 2 \Delta r c 3 \Delta$ was specific to the Rcf2 ${ }^{\mathrm{N}}$-Rcf1 construct (Figure 3-21). Both fragments of Rcf2 failed to enhance respiration significantly and are not functional as such. This is counterintuitive, yet not completely surprising. Römpler et al., (2016) made a similar discovery when they tested the functionality of Rcf2 fragments, after they imported them successfully into mitochondria. Even though the C-terminal fragment of Rcf2 ${ }^{63-224}$ could assemble into respiratory supercomplexes, it could not function as full-length Rcf2 and rescue the growth phenotype of the RCF2 RCF3 double mutant. And neither could the corresponding fragment Rcf2 ${ }^{1-62}$ accomplish that (Römpler et al., 2016).

In the end, it was an important observation, that the functionality of the fusion proteins did not rely exclusively on the Rcf2 fragments and was specific. Especially with these results in mind, it was intriguing that $\mathrm{Rcf} 2^{\mathrm{N}}$-Rcf1 possesses enough functionality to rescue the respiration phenotype of $\operatorname{rcf} 2 \Delta \operatorname{rcf} 3 \Delta$. 


\subsubsection{Oxygen consumption and ROS measurements reveal dynamic functions of fusion proteins}

Given the effect on respiration of the particular fusion proteins, we expected to obtain a detailed understanding of the mechanistic influence regarding the protein domains by measuring the respiration rate and ROS production. The RCF mutants displayed respirational phenotypes in terms of oxygen consumption on the one hand, and undesired release of reactive oxygen species (ROS) on the other hand (Vukotic et al., 2012; Römpler et al., 2016). Loss of Rcf1 causes a higher ROS production with concurrent oxygen consumption impairment and drop of complex IV activity (Vukotic et al., 2012). Rcf2 and Rcf3 revealed their overlapping regulatory role of complex IV while $\operatorname{rcf} 2 \Delta$ results in enhanced ROS production as well (Römpler et al., 2016).

Both assays are performed with isolated mitochondria in the same respiration medium which allows comparability. ROS production is detected, in this case, with AmplexUltra Red which reacts with $\mathrm{H}_{2} \mathrm{O}_{2}$ to fluorescent resorufin. $\mathrm{H}_{2} \mathrm{O}_{2}$ only represents a subset of ROS, yet $\mathrm{H}_{2} \mathrm{O}_{2}$ is formed when superoxide anions react with superoxide dismutase (SOD). Consequently, it is a sufficient indicator of ROS production in mitochondria. $\mathrm{H}_{2} \mathrm{O}_{2}$ concentration was measured with saturated amounts of non-phosphorylating supplements. Thus, measuring conditions are in principle similar to those during LEAK respiration. The different oxygen consumption states and their conditions were mentioned more in detail above in section 3.3.1.

As in the other analyses, isolated mitochondria from wild-type cells were applied for a first assessment. While mitochondria of wild-type cells expressing Rcf3-Rcf1 display a similar ROS level like wild-type mitochondria itself, overexpressing parts of Rcf2 lead to a significantly higher ROS production (Figure 3-22A). At the same time, oxygen consumption remains stable in the Rcf $2^{\mathrm{N}}$-Rcf1 containing strain, compared to wild-type (Figure 3-22B). Apparently, higher ROS production is not necessarily caused by higher respiration activity. Overexpression of Rcf3 due to the fusion proteins attenuates respiration capacity, especially together with $\mathrm{Rcf}^{\mathrm{C}}$ overexpression. Surprisingly, their effect is not bound to complex IV activity, although this seem to be their major interacting complex. The effect of Rcf3 overexpression implies to be more systemic in regard to the whole respirasome. 
A

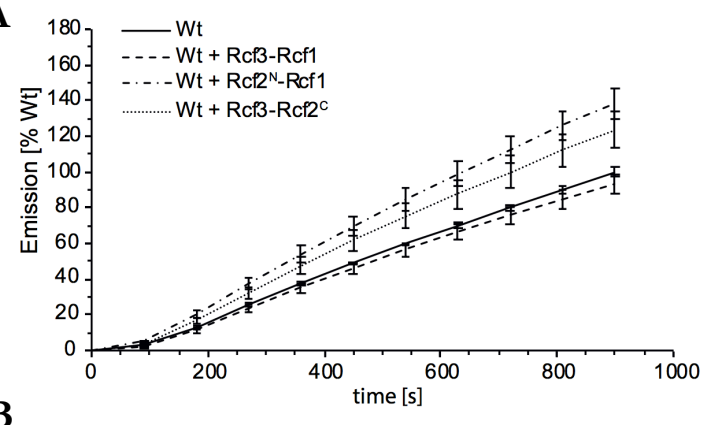

B

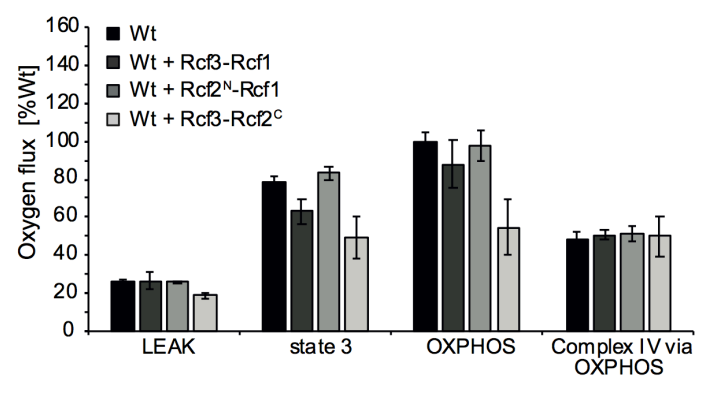

Figure 3-22: Overexpressing parts of Rcf2 results in higher ROS production.

A) Isolated mitochondria from wild-type (Wt) cells expressing different fusion proteins were applied for $\mathrm{H}_{2} \mathrm{O}_{2}$ concentration measurements with fluorescent dye AmplexUltra Red ( $n=3$ ). Emission (Exc: $555 \pm 10 \mathrm{~nm}$, Em: $581 \pm 5 \mathrm{~nm}$ ) was measured after adding HRP as catalysator. Normalization: $100 \%=\mathrm{Wt}(900 \mathrm{~s})$ B) Oxygen consumption measurement of same mitochondria as in A) with the Oxygraph-2k ( $n=3)$. LEAK: non-phosphorylating state; State3: upon addition of saturating ADP; OXPHOS: maximum respiration under coupled conditions; OXPHOS via CIV: complex IV activity in parallel to complex III inhibition. Normalization: $100 \%=\mathrm{Wt}(\mathrm{OXPHOS})$. Error bars indicate mean \pm SEM

Overall, overexpressing the Rcf-proteins as part of the fusion constructs appears to interfere with proper endogenous Rcf-protein function. The fact that Rcf3-Rcf1 and Rcf3-Rcf2C are behaving differently indicates that the domains still display a distinct functionality although fused to others.

Next, we were focusing on the effects of the fusion proteins within the mutants. With regard to comparability of results and dimension of the different measurements, they were normalized to the wild-type measurements. As it was also determined in a previous study, the RCF1 deletion mutant shows a significantly higher ROS production than the wild-type and a heavily impaired respiration (Vukotic et al., 2012). Rcf $2^{\mathrm{N}}$-Rcf1 appeared to be promising by modulating OXPHOS capacity as well as complex IV activity back to wild-type level in $r c f 1 \Delta$ (Figure 3-23C). The high ROS production of $r c f 1 \Delta$ is also decreased, however, it cannot rescue the phenotype completely (Figure 3-23A). Rcf3-Rcf1 shows the opposite behavior - although undesired ROS production can be prevented and is comparable to wild-type level (Figure 3-23B), the OXPHOS capacity is only moderately enhanced (Figure 3-23D). Complex IV activity can be restored and like in the wild-type situation overexpression of Rcf3 seem to impact respiration but not via complex IV. 


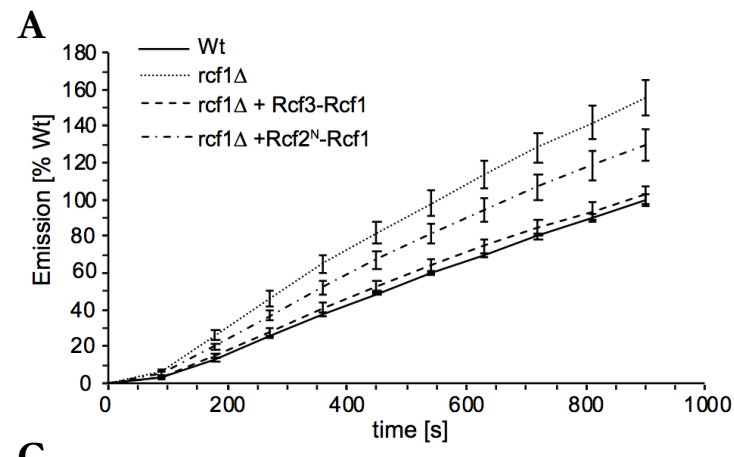

C

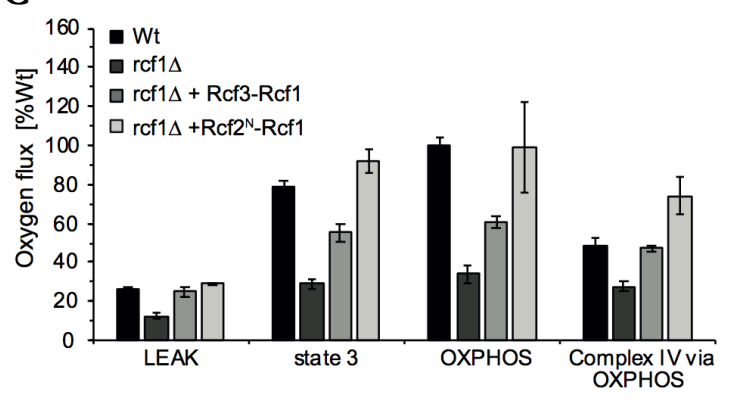

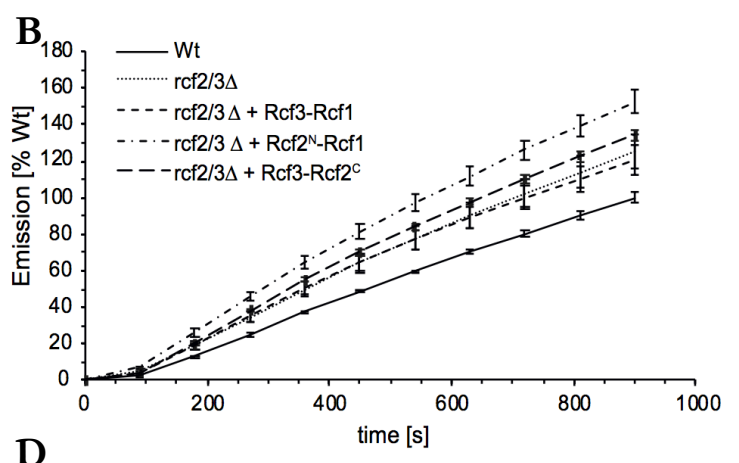

D

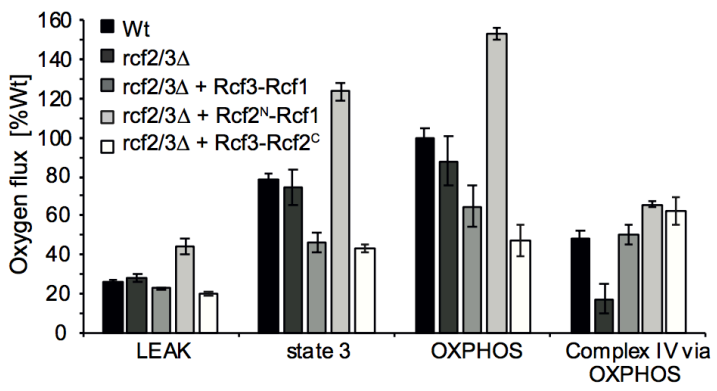

Figure 3-23 Fusion proteins modulate oxygen consumption and $\mathrm{H}_{2} \mathrm{O}_{2}$ production in a flexible way.

A) and B) Isolated mitochondria from $r f f 1 \Delta$ or $r f 2 \Delta r f 3 \Delta$ cells expressing different fusion proteins were applied for $\mathrm{H}_{2} \mathrm{O}_{2}$ concentration measurements with fluorescent dye AmplexUltra Red (n=3). Emission (Exc: $555 \pm 10 \mathrm{~nm}$, Em: $581 \pm 5 \mathrm{~nm}$ ) was measured after adding HRP as catalysator. Normalization: $100 \%=\mathrm{Wt}(900 \mathrm{~s})$. C) and D) Oxygen consumption measurement of same mitochondria as in A) and B) with the Oxygraph-2k ( $\mathrm{n}=3$ ). LEAK: non-phosphorylating state; State3: upon addition of saturating ADP; OXPHOS: maximum respiration under coupled conditions; OXPHOS via CIV: complex IV activity in parallel to complex III inhibition. Normalization: $100 \%=\mathrm{Wt}(\mathrm{OXPHOS})$. Wt control is identical through all experiments. Error bars indicate mean \pm SEM.

The double mutant did not show a drastic difference in respiration in general, yet complex IV activity is even more affected than in $r f 1 \Delta$. When Römpler and colleagues (2016) compared $r c 2 \Delta r f 3 \Delta$ respiration to the wild-type, respiration via complex IV was similarly affected but also respiration principally (Römpler et al., 2016). Our results, however, indicate that measured OXPHOS capacity and complex IV activity do not necessarily promote each other.

When we investigated the ROS level of $\operatorname{rcf} 2 \Delta \operatorname{rcf} 3 \Delta$ mitochondria, it was significantly higher than of wild-type mitochondria, yet not as high as of $\operatorname{rcfl} \Delta$ ((Figure 3-23A+C). Together with the observation that in contrast to $r f f 1 \Delta$, the respiratory supercomplex arrangement is not altered, the results confirm the different nature of both mutants. Nevertheless, the endpoints as complex IV activity, ROS production and growth on non-fermentable media appear similar.

Neither of the fusion proteins were able to lower the ROS production in the double mutant (Figure 3-23B). Rcf2 ${ }^{\mathrm{N}}$-Rcf1 even enhanced the ROS level significantly. At the same time, this protein version displayed the highest OXPHOS capacity of all measured mitochondria, which was paradoxically not mediated through a significantly increased complex IV activity compared 
to wild-type (Figure 3-23D). In parallel, the LEAK respiration was enhanced, indicating a proton leakage through the inner mitochondrial membrane, which might be even more pronounced upon addition of supplements addressing the OXPHOS state.

The fusion proteins comprising Rcf3 show in general a downregulation of oxygen consumption, which means that the domains fused to each other cannot fulfill the functions of endogenous Rcf2 or Rcf3. They rather interfere with their intrinsic determination.

In the end our results demonstrated that the artificial fusion constructs can act very flexibly depending on the requirements when one or several Rcf-proteins are not present. However, it revealed also, that the constructs are not acting as the respective full-length endogenous proteins and still harbor distinct functionalities. After this general assessment of mitochondrial function, it was important to furthermore examine the influence on specific interaction sites. All endogenous Rcf-proteins were found to bind to newly synthesized complex IV subunits (section 3.1.3), while Rcf1 was reported as potential player in cytochrome $c$ oxidase maturation (Strogolova et al., 2012; Garlich et al., 2017). Consequently, we were wondering, whether the fusion constructs can influence this function in a similar flexible manner.

\subsubsection{Fusion proteins influence Rcf1 association with newly translated Cox3}

To characterize the role of transmembrane domains with respect on the involvement of Rcf1 during complex IV maturation, another labeling approach with isolated mitochondria was conducted. Upon radioactive labeling of mitochondrial translated proteins with ${ }^{35} \mathrm{~S}$-Metionine, the co-immunoprecipitation followed with Rcf1 specific antiserum to isolate Rcf1 specific complexes. First, mitochondria from wild-type cells expressing the different artificial proteins were investigated. The assay was performed as before in section 3.1.3. with a ${ }^{35} \mathrm{~S}$-Met pulse labeling of $10 \mathrm{~min}$ and the solubilization of mitochondria with DDM prior to co-immunoprecipitation. 
A

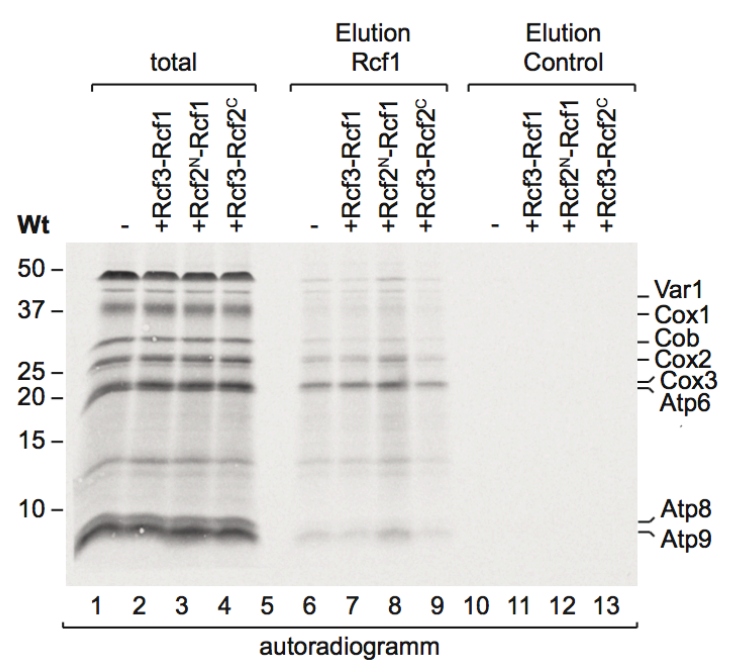

C

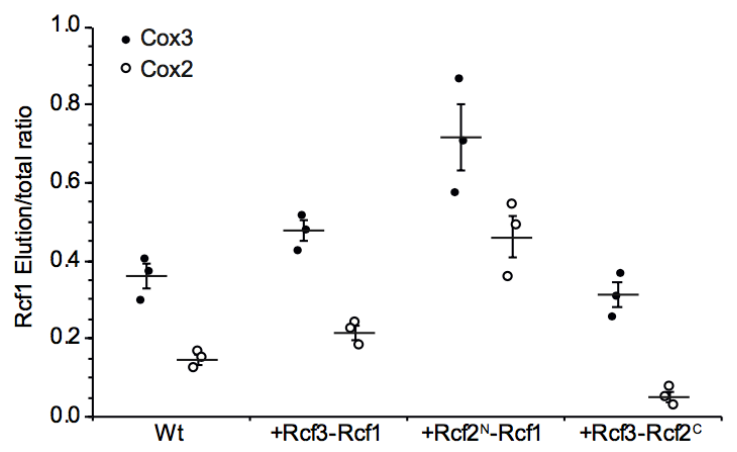

B

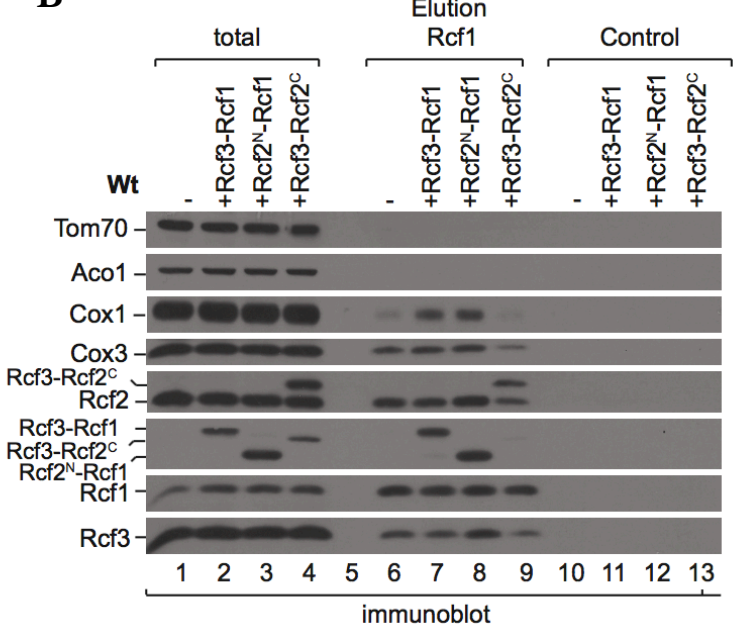

Figure 3-24: Rcf3-Rcf1 and Rcf2N-Rcf1 support post-translational interaction with Cox3.

A) Isolated mitochondria from wild-type (Wt) cells expressing the indicated fusion proteins are subjected to radioactive labeling of mitochondrial encoded proteins with ${ }^{35} \mathrm{~S}$-methionine for $10 \mathrm{~min}$, followed by solubilization with $0.6 \%$ DDM and co-immunoprecipitation with Rcf1 and control beads. Totals and glycine elution were subjected to Tricine-SDS PAGE (10-18 \%), western blotting and autoradiogram. B) Immunodetection of A). Tom70 and Aco1 serve as control proteins for specific isolation. C) Quantification of isolated $\operatorname{Cox} 3$ and $\operatorname{Cox} 2$ from A) with $n=3$. Results are normalized to the respective total signals and were quantified using ImageJ software with background subtraction (Schindelin et al., 2012). Error bars indicate mean \pm SEM.

It seemed to be intuitive that proteins harboring extra Rcf1, co-purify more labeled Cox3 (Figure $3-24 \mathrm{~A}+\mathrm{C}$ ). Furthermore, Cox2 was also co-isolated specifically as mentioned before (section 3.1.3), but apparently in a different ratio in the Rcf3-Rcf2 ${ }^{\mathrm{C}}$ strain compared to the other strains. This implies that there is a ratio shift of Rcf1 interacting exclusively with the COX3 module, but not with mature complex IV. This could also be determined via immunodetection (Figure 3-24B), where Cox1 is specifically co-isolated with Rcf1 when Rcf3-Rcf1 and Rcf2 ${ }^{\mathrm{N}}$-Rcf1 are present. In parallel, other interactors of Rcf1 are eluted in a decreased concentration as it is the case for Cox3, Rcf2, and Rcf3, while purification efficiency of Rcf1 remained stable. It indicates that Rcf3-Rcf2 ${ }^{\mathrm{C}}$ rather interferes while Rcf3-Rcf1 and Rcf2 ${ }^{\mathrm{N}}$-Rcf1 support endogenous Rcf1 function in terms of post-translational Cox 3 binding and its modular assembly. But it could also be a result of the different expression levels, as mentioned before (section 3.4.1). 
A

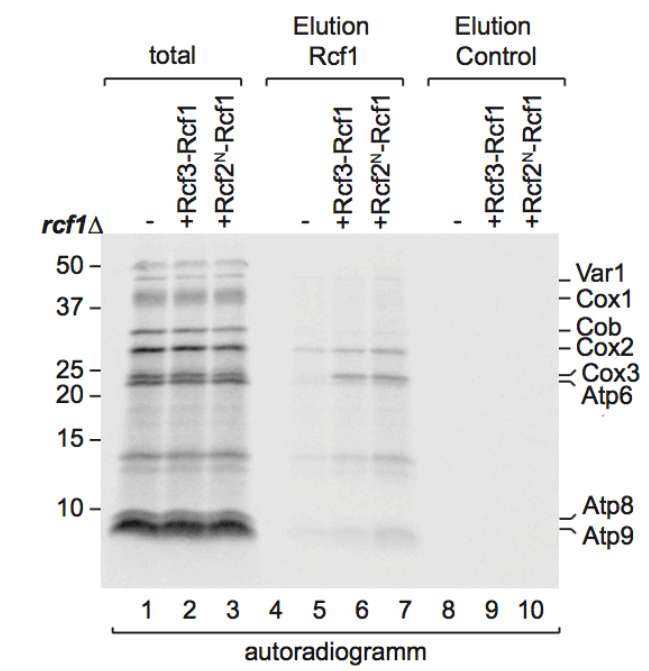

C

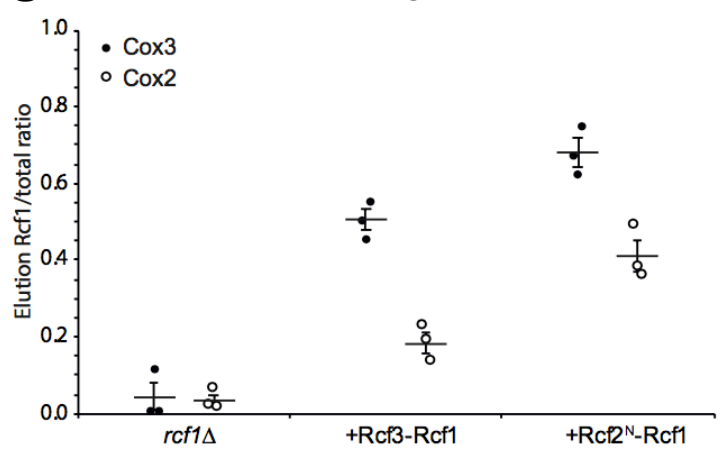

B
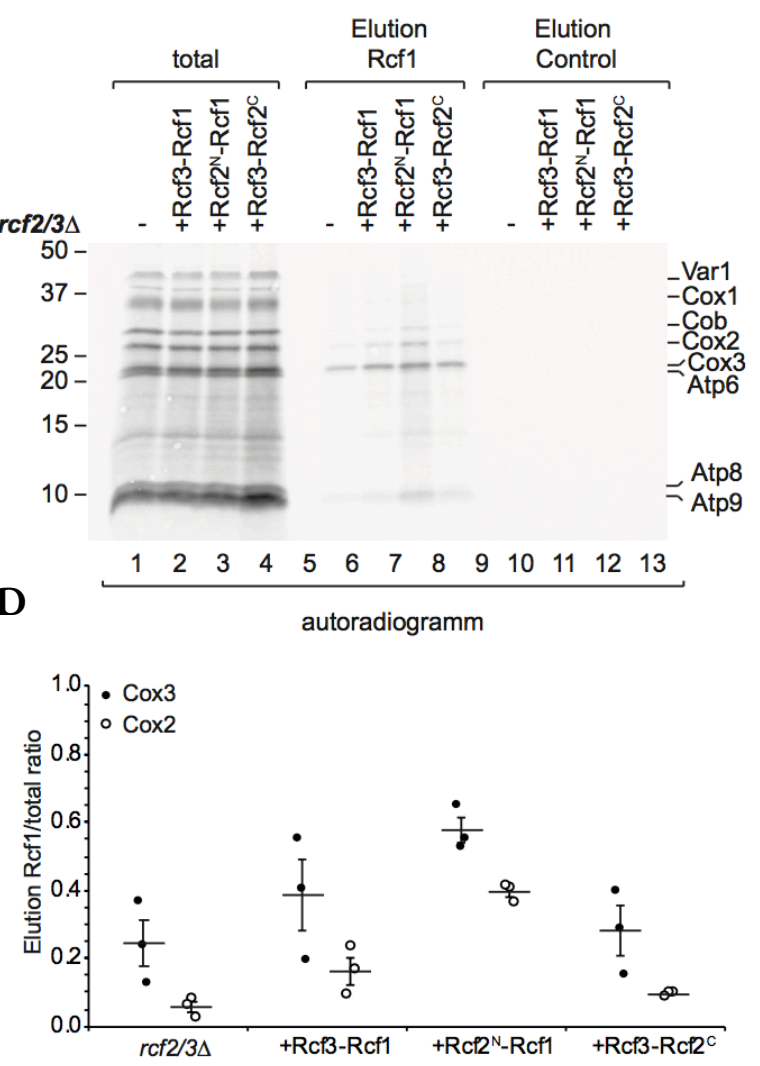

$\mathbf{E}$
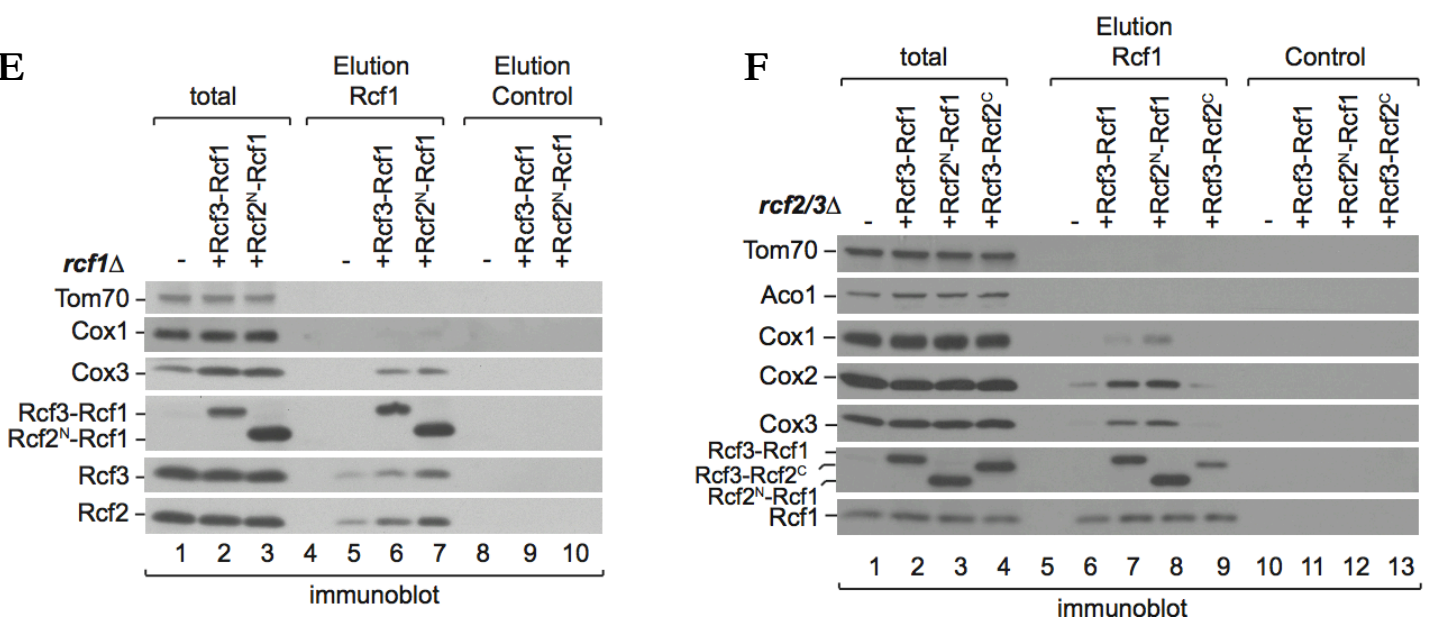

Figure 3-25: Post-translational interaction of Rcfl with Cox3 is impaired in rcf2 $\triangle r c f 3 \Delta$.

A) and B) Isolated mitochondria from $r c f 1 \Delta$ or $r f 2 \Delta r f 3 \Delta$ cells expressing the indicated fusion proteins are subjected to radioactive labeling of mitochondrial encoded proteins with ${ }^{35} \mathrm{~S}$-methionine for $10 \mathrm{~min}$, followed by solubilization with $0.6 \%$ DDM and co-immunoprecipitation with Rcf1 and control beads. Totals and glycine elution were subjected to Tricine-SDS PAGE (10-18 \%), western blotting and autoradiogram. C) and D) Quantification of isolated Cox3 and Cox2 from A) with $\mathrm{n}=3$. Results are normalized to the respective total signals and were quantified using Image software with background subtraction (Schindelin et al., 2012). Error bars indicate mean \pm SEM. E) and F) Immunodetection of A). Tom70 and Aco1 serve as control proteins for specific isolation.

Next, we wanted to study those effects of the respective plasmids encoding for the fusion proteins when transformed into the mutants $\operatorname{rcf} 1 \Delta$ and $\operatorname{rcf} 2 \Delta r f 3 \Delta$. The fusion proteins containing Rcf1 co-isolated more radioactively labeled Cox3 and Cox2 from $\operatorname{rcf} 2 \Delta r f 3 \Delta$ as well 
(Figure 3-25B+D), but not as effective as from $r c f 1 \Delta$ (Figure 3-25A+C) or before from wild-type mitochondria (Figure 3-24A+C). Already the situation of the double mutant itself distinguishes from the wild-type situation. Apparently, deletion of $\mathrm{RCF} 2$ and $\mathrm{RCF} 3$ affects the post-translational interaction of Rcf1 with Cox3. The immunodetection of Cox3 displays an affected interaction with Rcf1 as well, including the overall accessible Cox3 from complex IV. This might stand in relation to the proposed mutual role of Rcf1 and Rcf2 in Cox3 assembly and/or folding (Strogolova et al., 2019).

Still, the effect of the fusion proteins in the $r f 2 \Delta r c f 3 \Delta$ background is not as different, considering the initial level of interaction. The existence of Rcf2 fragments or Rcf3, yet seem to interfere with Rcf1 function when endogenous Rcf2 and Rcf3 are not present. The interaction of solely Rcf3-Rcf1 and Rcf2 ${ }^{\mathrm{N}}$-Rcf1 with newly synthesized Cox3 in the $r f 1 \Delta$ mutant is comparable to the wild-type situation, overexpressing at least parts of the Rcf-proteins. This means that the overexpression itself does not have an effect in this regard but the domains fused to each other. Those assays confirmed again the dynamic and flexible role the fusion proteins can occupy.

Given that the Rcf-protein family obviously interacts with already maturing cytochrome $c$ oxidase, we were moreover aiming to enrich the proposed state of interaction at the site of Cox 3 and Cox2. For this, the deletion of Cox 5a seemed to be a useful tool. Cox 5 a represents an early assembling protein of the COX1 module (section 1.4.3) (Fontanesi et al., 2006; Mick et al., 2007). A deletion consequently results in disrupted COX1 modular assembly and the other modules, more relevant in regard to Rcf-proteins, can be studied more independently. On the other hand, Hartley et al. (2020) could resolve a Rcf2 association with complex $\mathrm{III}_{2} \mathrm{IV}$ in a cox $5 a \Delta$ condition via cryo-EM. Thus, we were curious about a possible influence on Rcf-protein association at complex IV when Cox $5 \mathrm{a}$ is absent.

\subsection{Interaction of Rcf2 with complex III}

\subsubsection{Cox $5 a \Delta$ reveals specific interaction of Rcf2 with complex $\mathrm{II}_{2}$}

Working with $\cos 5 a \Delta$ or $\cos 5 b \Delta$ yields the advantage that there is no mixed population of hypoxic and normoxic supercomplexes as the proteins represent oxygen dependent isoforms (see also section 1.4.4). This is usually ignored under wild-type conditions, where also small amounts of the hypoxic isoform are expressed (Figure 3-26A). A steady state analysis of $\operatorname{cox} 5 a \Delta$ and $\operatorname{cox} 5 b \Delta$ mitochondria showed, that the $\operatorname{cox} 5 b \Delta$ does not display a significant change 
of either complex IV or complex III subunits on a protein level, compared to wild-type (Figure 3-26A). Since Cox5b is expressed only in minor amounts in a wild-type situation, it is expected that its deletion does not have a strong impact. Cox5a mitochondria, in contrast, display a heavy decrease in complex IV subunits. The reason is probably the expression level of Cox $5 \mathrm{~b}$. In a $\operatorname{cox} 5 a \Delta$ situation the level of the hypoxic isoform is only slightly increased and cannot compensate for loss of Cox5a. Complex III subunits like Rip1 and Qcr8 as well as the Rcf-proteins are not significantly affected (Figure 3-26A).

A
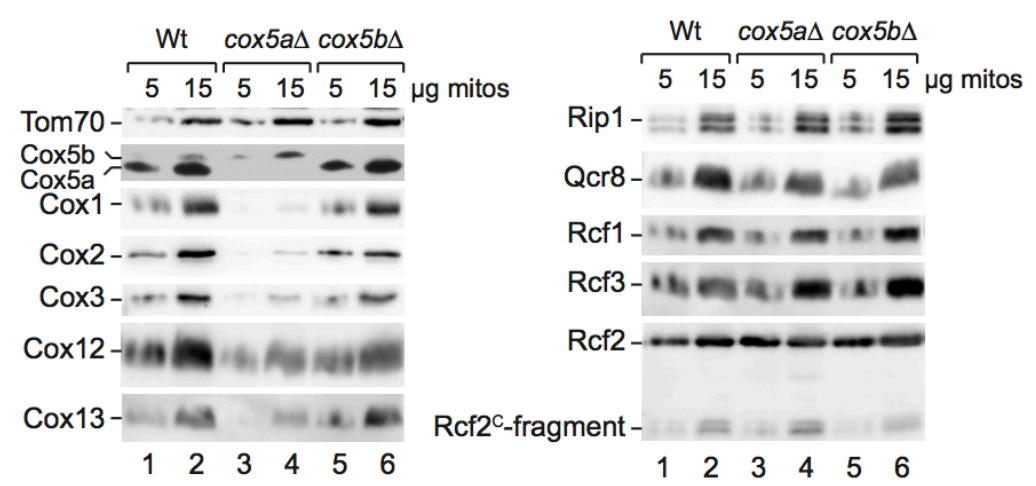

B

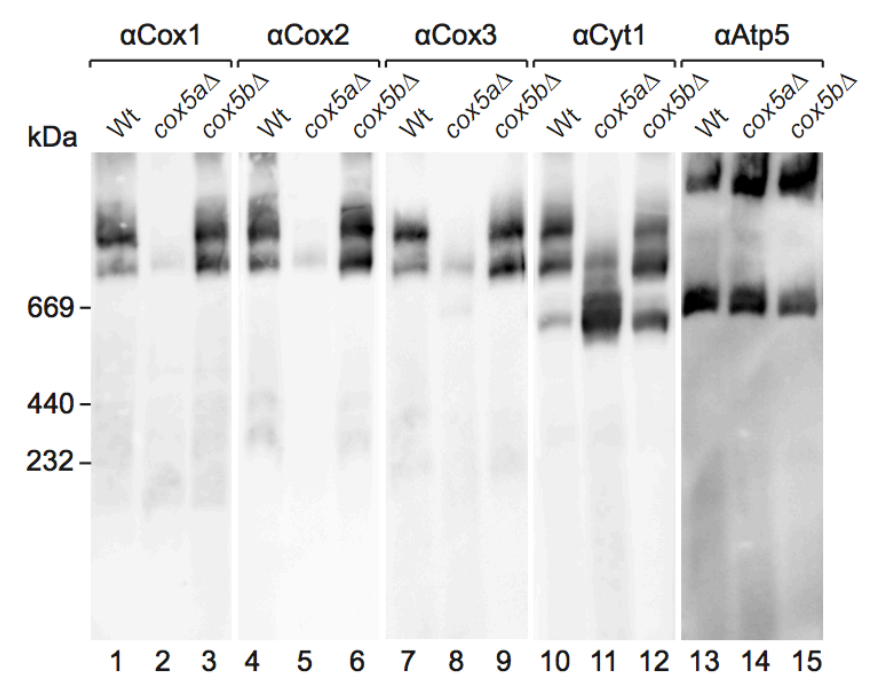

Figure 3-26: Hypoxic isoform Cox5b cannot compensate for loss of Cox5a.

A) Isolated wild-type (Wt), cox5 $a \Delta$ and $\operatorname{cox} 5 b \Delta$ mitochondria were lysed in Laemmli buffer and applied to SDS-PAGE (10-16 \%) and Urea SDS PAGE (17.5\%) for resolving Cox5a and Cox5b. Rip1 and Qcr8 represent complex III subunits, Cox-antibodies complex IV. B) Digitonin solubilized mitochondria from Wt, cox5a $\Delta$ and $c 0 \times 5 b \Delta$ cells were subjected to BN-PAGE (4-10 \%). Complex IV is detected via Cox1, Cox2 and Cox3, complex III via Cyt1 and Atp5 serves as loading control detecting complex $\mathrm{V}$.

This result is also reflected by BN analysis. Since only minor amounts of Cox $5 \mathrm{~b}$ are present, supercomplex assembly is almost completely abolished (Figure 3-26B). Small amounts of complex $\mathrm{III}_{2} \mathrm{IV}$ are present, however the majority of complex III, displayed by Cyt1 detection, 
is found as dimeric $\mathrm{III}_{2}$. At the same time, cox5b shows almost wild-type like supercomplex formation.

To further investigate the association of Rcf-proteins in the $\operatorname{cox} 5 a \Delta$ situation, a $2^{\text {nd }}$ dimension analysis after BN-PAGE was conducted in comparison to wild-type mitochondria (Figure 3-27). It confirmed the first assessments of the $1^{\text {st }}$ dimension, with the majority of complex III being present as a dimer, but small amounts of supercomplex $\mathrm{III}_{2} \mathrm{IV}$ being built in $\operatorname{cox} 5 a \Delta$. In the wild-type analysis (Figure 3-27A), previous observations could be confirmed with all three Rcf-proteins residing at complex $\mathrm{III}_{2} \mathrm{IV}_{(1-2)}$ (Vukotic et al., 2012; Römpler et al., 2016). Yet, the majority of Rcf1, Rcf2 and Rcf3 co-migrates in smaller complexes.

A

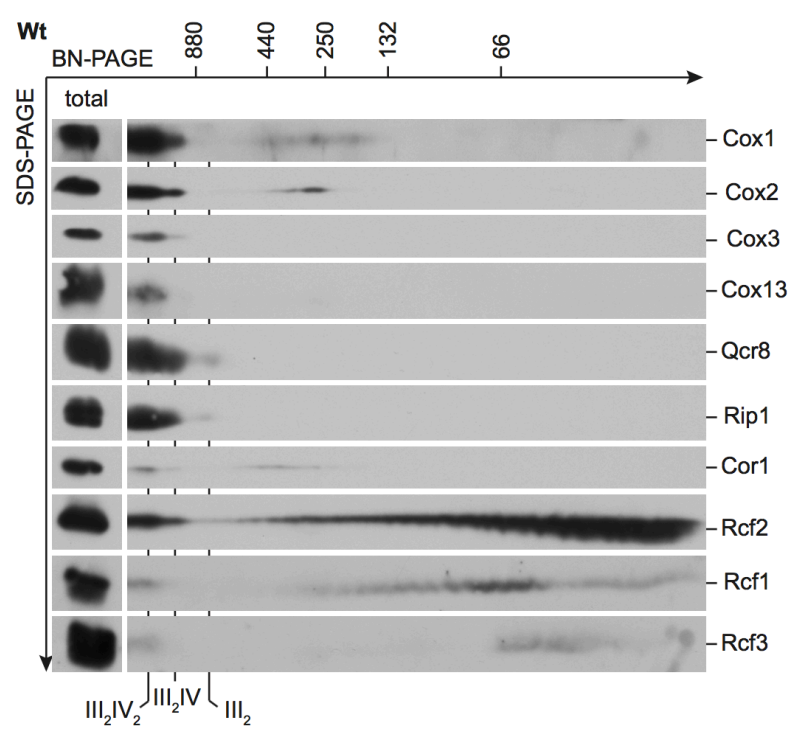

B

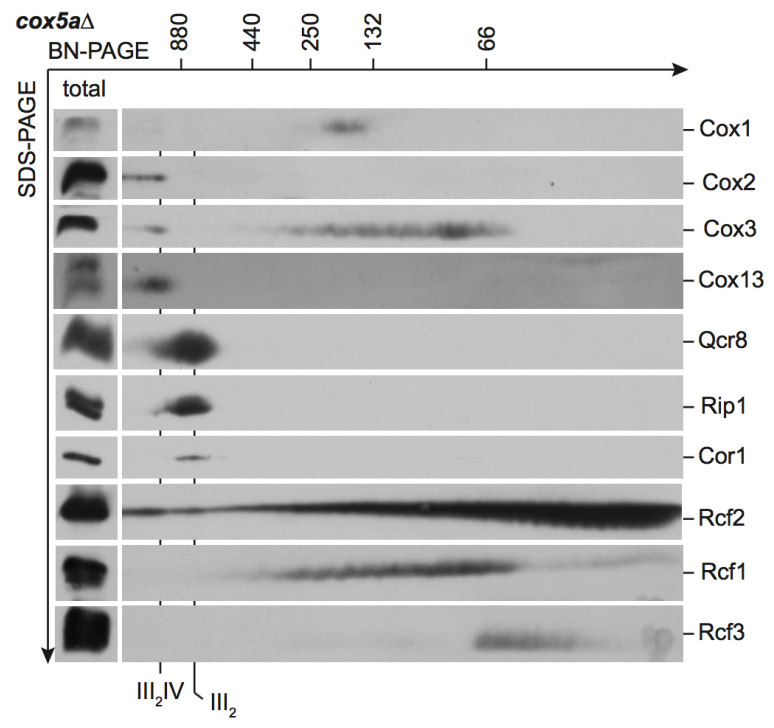

Figure 3-27: Rcf2 associates with complex $\mathrm{III}_{2}$ in $\operatorname{cox} 5 \mathrm{a} \Delta$ while supercomplex assembly is impaired.

A) and B) Digitonin solubilized mitochondria from wild-type (Wt) or cox5a cells, respectively, were subjected to BN-PAGE (4-16\%) and the lane was used for $2^{\text {nd }}$ dimension SDS-PAGE (16\%) and western blotting. Complex IV is represented by Cox1, Cox2, Cox3 and Cox13 detection, complex III by Rip1, Cor1 and Qcr8.

Remarkably, Rcf2 could be detected as residing at the supercomplex and complex $\mathrm{III}_{2}$ in $\operatorname{cox} 5 a \Delta$ as well (Figure 3-27B). Although the ability of Rcf2 to bind with complex III was shown previously (Römpler et al., 2016), a significant detection at complex $\mathrm{III}_{2}$ has not been experienced yet. Rcf1 and Rcf3 are barely detectable at higher molecular weight complexes in the $\operatorname{cox} 5 a \Delta$ situation. While the majority of Rcf3 is migrating at around $66 \mathrm{kDa}$, Rcf1 is resolved similarly to Cox3. The migration of the smaller Rcf-complexes, however, does not change significantly when Cox5a is missing.

It seems a bit contradictory, on the one hand having Rcf2 resolved in complex $\mathrm{III}_{2} \mathrm{IV}$ at the periphery of complex IV and on the other being able to detect it at complex $\mathrm{III}_{2}$, both in $\operatorname{cox} 5 \mathrm{a} \Delta$, an alleged hypoxic state. Only the C-terminus of Rcf2 was resolved within the cryo-EM 
structure (Hartley et al., 2020), therefore we were questioning a specific involvement of the distinct Rcf2 domains. On the one side half of Rcf2 could be responsible for complex $\mathrm{III}_{2}$ association, while the $\mathrm{C}$-terminus of Rcf2 is crucial for $\mathrm{III}_{2} \mathrm{IV}$ interaction under these conditions. Consequently, we utilized once more the verified tool of Rcf fusion proteins with the intention to study the relevance of the different transmembrane domains for the interaction.

\subsubsection{Behavior of fusion proteins in $\operatorname{cox} 5 a \Delta$}

Upon expression of Rcf3-Rcf1, Rcf2 ${ }^{\mathrm{N}}$-Rcf1 and Rcf3-Rcf2 ${ }^{\mathrm{C}}$ in $\operatorname{cox} 5 a \Delta$ cells, we addressed if the localization of the constructs changed from a wild-type situation (section 3.4.2) and conducted $2^{\text {nd }}$ dimension analysis after BN-PAGE. Complex $\mathrm{III}_{2} \mathrm{IV}$ is represented by complex IV subunits Cox1, Cox2 and Cox13 and complex III subunits Rip1 and Qcr8 (Figure 3-28).

Although only small quantities of complex $\mathrm{III}_{2} \mathrm{IV}$ are built, the constructs can be determined at the supercomplexes, except for Rcf2 ${ }^{\mathrm{N}}-$ Rcf1 (Figure 3-28B). However, the protein amounts detectable in this case are low, and apparently, neither of the proteins behave like endogenous Rcf2 and reside at complex $\mathrm{III}_{2}$. Like before, the majority of Rcf1 and Rcf3 migrate at lower molecular weight complexes in this background. Nevertheless, the fusion construct of Rcf3 and Rcf1 is detectable at $\mathrm{III}_{2} \mathrm{IV}$ (Figure 3-28A), leading to the assumption that the topology itself plays an important role in the complex association.

In addition, we were paying attention to the processed versions of Rcf2, since these represent the single transmembrane regions descending from endogenous Rcf2. Thus, investigating the Rcf2 fragments might reveal a specific interaction site for the transmembrane regions which we could not detect with the fusion proteins. Exclusively the endogenous C-terminal of Rcf2 is recognized by the antibody. Interestingly, we could determine that it co-localizes like full-length Rcf2 at both complex $\mathrm{III}_{2} \mathrm{IV}$ and $\mathrm{III}_{2}$ (Figure 3-28). This raised the question, how the internal processing event could be related to this unexpected behavior. By tracing the processed versions of Rcf2 we were aiming not only for understanding the association of Rcf2 but also for identifying the conditions under which processing is triggered. 
A

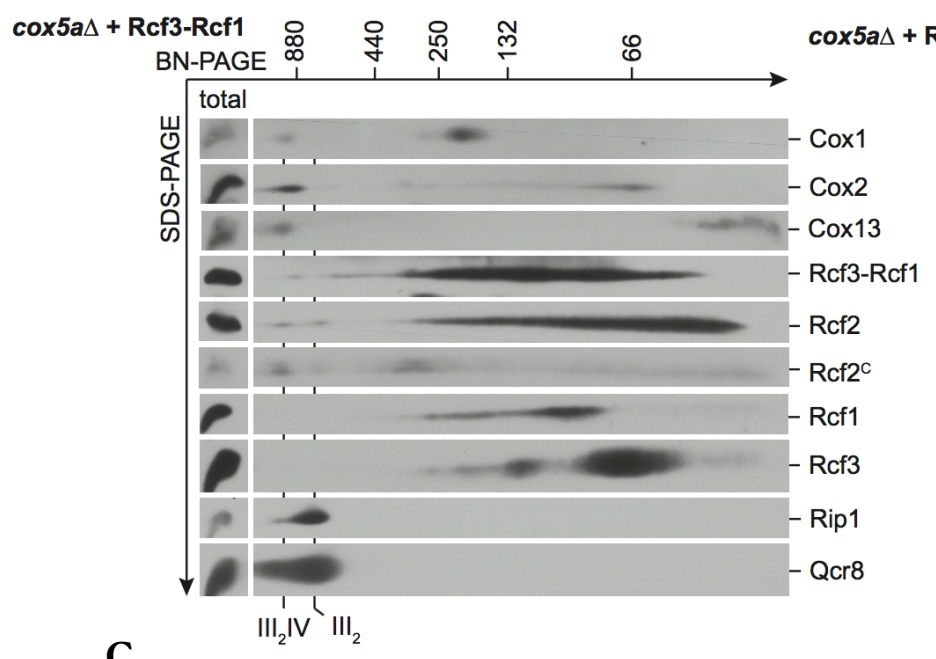

C

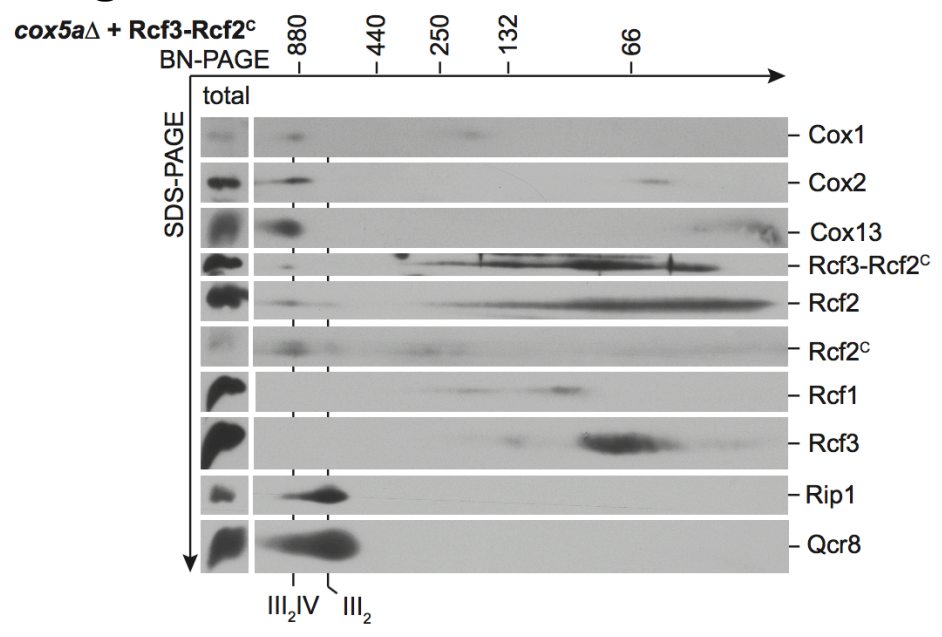

B

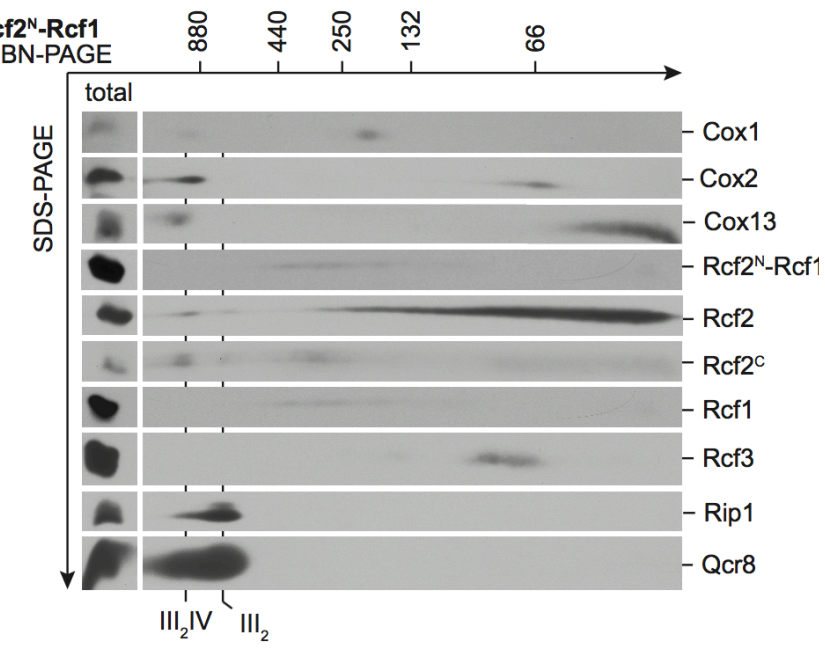

Figure 3-28: The minority of fusion proteins resides at $\mathrm{III}_{2} \mathrm{IV}$ and $\mathrm{Rcf}^{\mathrm{C}}$ co-migrates with $\mathrm{III}_{2}$.

A) Digitonin solubilized mitochondria from $\operatorname{cox} 5 a \Delta$ cells expressing Rcf3-Rcf1 were subjected to BN-PAGE (4$16 \%$ and the lane was used for $2^{\text {nd }}$ dimension SDSPAGE $(16 \%)$ and western blotting. Complex IV is represented by Cox1, Cox2 and Cox13 detection, complex III by Rip1 and Qcr8. B) and C) as in A) with mitochondria from $\operatorname{cox} 5 a \Delta$ cells expressing Rcf $2^{\mathrm{N}}-\mathrm{Rcf1}$ and Rcf3-Rcf2C, respectively.

\subsubsection{Tracing processed Rcf2 in the $\operatorname{cox} 5 a \Delta$ background}

The fact that Rcf $2^{\mathrm{C}}$ can be observed to associate with complex $\mathrm{III}_{2}$ in a $\operatorname{cox} 5 \mathrm{a} \Delta$ situation, raised the question, what the role of the more labile $\mathrm{Rcf} 2^{\mathrm{N}}$ could be. We were furthermore wondering if the co-localization differs from full-length Rcf2 or Rcf2 ${ }^{C}$. When Römpler et al. (2016) first studied the processed versions of Rcf2 under wild-type conditions, they found Rcf2 ${ }^{\mathrm{C}}$ still associated to the supercomplexes, while Rcf2 $2^{\mathrm{N}}$ could not be detected to interact with them. For studying the N-terminal fragment they introduced a FLAG-tagged version of Rcf2 ( ${ }^{\mathrm{FLAG}} \mathrm{Rcf} 2$ ). By this, Rcf2 ${ }^{\mathrm{N}}$ detection was possible via FLAG antibody (Römpler et al., 2016). We adopted this approach and ${ }^{\mathrm{FLAG}} \mathrm{Rcf} 2$ was expressed from a centromeric plasmid and transformed into the $\operatorname{cox} 5 a \Delta \operatorname{rrf} 2 \Delta$ and $\operatorname{cox} 5 a \Delta$ background. First, we determined, if both fragments, Rcf2 ${ }^{\mathrm{C}}$ and $\mathrm{Rcf}^{\mathrm{N}}$ can be isolated together with Qcr8 in $\operatorname{cox} 5 a \Delta$. To exclude that the expression of ${ }^{\mathrm{FLAG}} \mathrm{Rcf} 2$ has an effect on the interaction, $c 0 \times 5 a \Delta$ with endogenous expression of Rcf2 served as a control. It appeared that expression of ${ }^{\mathrm{FLAG}} \mathrm{Rcf} 2$ is increased, compared to wild-type Rcf2. The FLAG-tagged version also seems to promote processing of Rcf2, which can be detected via 
Rcf2 antibody (Figure 3-29A). Both versions are purifiable via Qcr8 co-immunoprecipitation, considering that the majority of Qcr8 is present in complex $\mathrm{III}_{2}$. In addition, the remaining ${ }^{\text {FLAG }} \mathrm{Rcf} 2{ }^{\mathrm{N}}$ fragment is associated with Qcr8. When isolating from the FLAG tag, ${ }^{\mathrm{FLAG}} \mathrm{Rcf2}$ can be enriched together with small amounts of ${ }^{\mathrm{FLAG}} \mathrm{Rcf} 2{ }^{\mathrm{N}}$ and sufficient amounts of Qcr8 (Figure 3-29B). The immunoprecipitations from both sides - Rcf2 and complex III, confirm the previous proposition with a specific Rcf2 interaction with complex III. However, the evidence was still circumstantial that this happens at complex $\mathrm{III}_{2}$ because $2^{\text {nd }}$ dimension analysis demonstrates a co-migration and not necessarily a complex association. While isolating from Qcr8 and ${ }^{\mathrm{FLAG}} \mathrm{Rcf} 2$, we cannot distinguish between $\mathrm{III}_{2} \mathrm{IV}$ and $\mathrm{III}_{2}$ complexes.

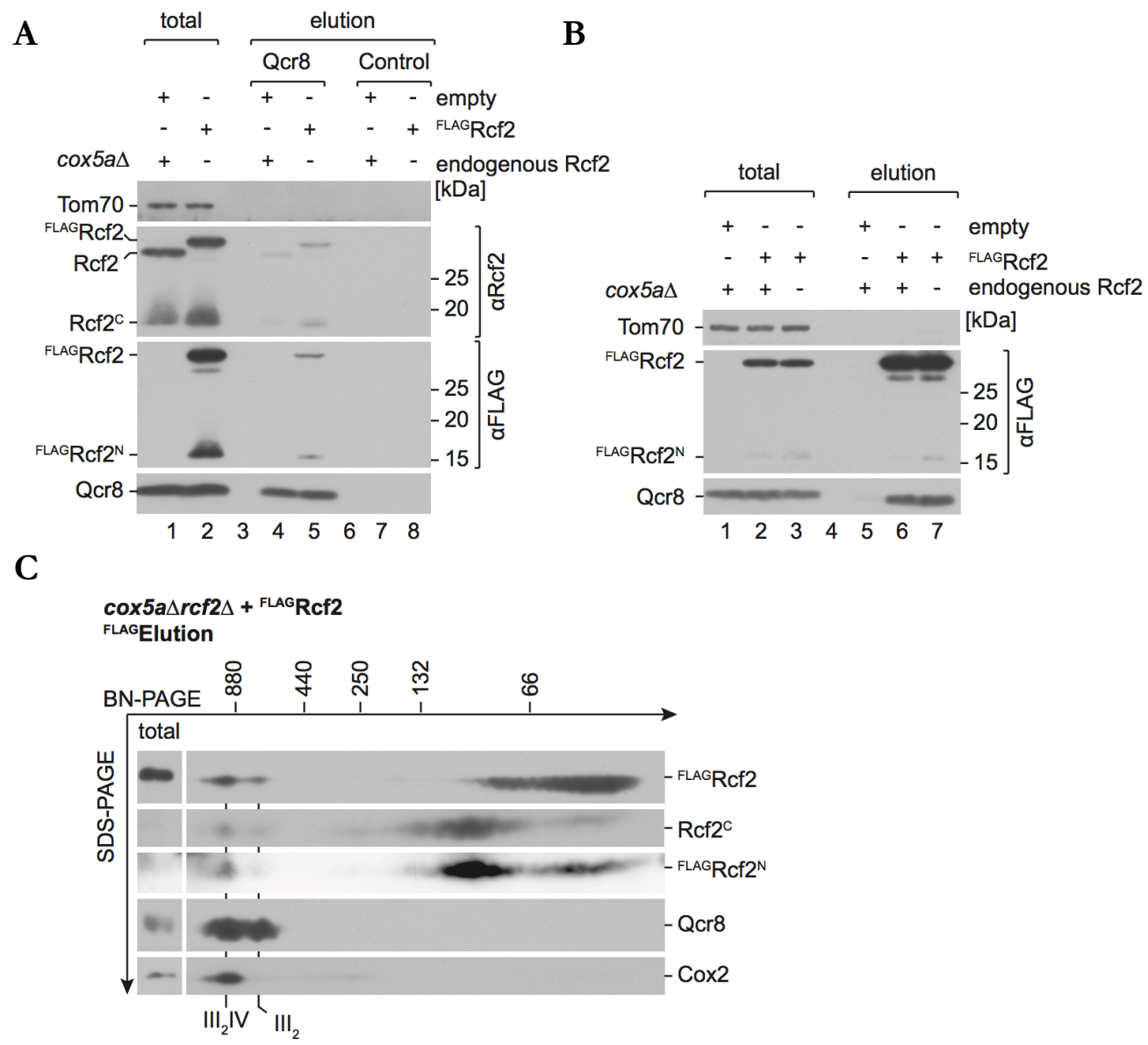

Figure 3-29: Processed versions of Rcf2 associate with complex $\mathrm{III}_{2} \mathrm{IV}$ and $\mathrm{III}_{2}$.

A) Digitonin (1\%) solubilized mitochondria from $\operatorname{cox} 5 a \Delta$ and $\operatorname{cox} 5 a \Delta r f 2 \Delta$ cells expressing FLAGRcf2, as indicated, where applied to Qcr8 co-immunoprecipitation. Totals and glycine eluates were analyzed via SDS-PAGE (10-16\%) and western blotting. Tom70 decoration and control columns prove specific interaction. B) Same mitochondria as in A) were solubilized with $1 \%$ Digitonin and used for FLAG-immunoprecipitation. Native elution was conducted with FLAG-peptide. Totals and eluates were analyzed via SDS-PAGE (10 16\%) and western blotting. Cox5a $\Delta$ mitochondria and Tom70 decoration serve as negative controls for specific isolation via FLAG-tag. C) As in B) with digitonin (1\%) solubilized mitochondria from cox5a $\operatorname{rcf} 2 \Delta$ cells expressing FLAGRcf2. Sample was subjected to BN-PAGE (4-16 \%) and the lane was used for $2^{\text {nd }}$ dimension SDS-PAGE (16\%) and western blotting. Complex IV is detected via Cox2 and complex III via Qcr8. FLAGRcf2 and FLAGRcf2N are detected via FLAG antibody and $\mathrm{Rcf}^{\mathrm{C}} \mathrm{C}$ via $\mathrm{Rcf} 2$ antibody. 
Consequently, we were further examining the hypothesis that the isolated complexes are in fact complex $\mathrm{III}_{2}$ by deploying the $2^{\text {nd }}$ dimension analysis in combination with native FLAG isolation. To this end, we could obtain the FLAG eluate enriches complex $\mathrm{III}_{2} \mathrm{IV}$ artificially, where the greater proportion of the Rcf2 versions is localized (Figure 3-29C). Interestingly, we could prove both fragments and full-length Rcf2 reside at complex $\mathrm{III}_{2} \mathrm{IV}$ and complex $\mathrm{III}_{2}$. Even the ratio of localization appears to be similar with the majority being present at $\mathrm{III}_{2} \mathrm{IV}$.

Thus, the fragments did not show a different accumulation in this analysis and we cannot conclude a specific interaction via one part of Rcf2 or the other. Yet, the interaction appears to descend from the initial interaction of full-length Rcf2. The sequence of that particular association remains unclear. Two scenarios are possible: the association with complex $\mathrm{III}_{2}$ happens due to degradation of the supercomplex $\mathrm{III}_{2} \mathrm{IV}$ or it occurs during an upstream process towards supercomplex assembly. Additionally, it is elusive if this finding stands in direct relation to $\operatorname{cox} 5 a \Delta$ and the alleged hypoxic state or whether the accumulation is caused by disabled supercomplex assembly.

Overall, it was a remarkable and surprising result to find Rcf2 and its fragments additionally at complex $\mathrm{III}_{2}$ considering its preferred association with complex IV (Vukotic et al., 2012; Römpler et al., 2016). This brings Rcf2 again into discussion as a true respiratory supercomplex assembly factor. 



\section{Discussion}

A vast effort has been made over the past century to shed more light into the dynamics of the mitochondrial respiratory chain. The view on the multimeric complexes made progress from the "solid-state" model towards a "plasticity" model. By now, it is a well-accepted theory that the respiratory chain is dynamically organized in so-called supercomplexes. Over the recent years, more and more valuable data has been gathered about the structure of the respiratory supercomplexes and helped to identify unknown interactors and subunits of the cytochrome $c$ oxidase and cytochrome $b c_{1}$ complex.

Novel cryo-EM structures of yeast respiratory supercomplexes revealed a close interaction between complex IV and complex III forming a connecting bridge via Cox $5 \mathrm{a} / \mathrm{b}$ and Cor1 (Hartley et al., 2019, 2020; Rathore et al., 2019). However, the mechanism and regulation of supercomplex assembly remains elusive. At this point, the Rcf-proteins came into attention as potential players in yeast supercomplex assembly. Rcf1 was found to be essential for efficient respirasome assembly (Chen et al., 2012; Vukotic et al., 2012). In parallel, there is evidence that the crucial interaction already takes place at the level of complex IV modular assembly. Rcf2 and Rcf3 were assigned to be involved in more regulatory pathways of complex IV and the supercomplex (Strogolova et al., 2012, 2019; Römpler et al., 2016; Garlich et al., 2017; Dawitz et al., 2020). However, the key interactors and localization were still ambiguous at the starting point of this study. In the end, our investigations aimed towards a better understanding of the role of the Rcf-proteins with respect to supercomplex assembly and their functional environment.

\subsection{Topology and orientation of the Rcf-proteins}

One of the first and basic observations we made was the relative orientation of the Rcf-proteins within the inner mitochondrial membrane. Previous studies claimed a $\mathrm{N}_{\text {out }}-\mathrm{C}_{\text {out }}$ orientation for Rcf1 and Rcf2. On the one hand with different detection methods targeting the different termini of the proteins (Chen et al., 2012; Strogolova et al., 2012; Vukotic et al., 2012) and on the other with in silico analysis (Römpler et al., 2016). The cryo-structure of Hartley et al. (2020) resolves the C-terminus of Rcf2 as facing towards the IMS and represents strong evidence for this model as well. Our proteinase accessibility analysis (section 3.1.1) confirms the C-termini of the three proteins protruding into the IMS. By including the analysis of the N-terminal FLAG-tagged version of Rcf2 we could find - once more - evidence for the N-terminus of Rcf2 being accessible from the IMS and at the same time verify the idea of an even number of 
transmembrane segments. Additionally, the crosslinking data acquired in collaboration with Linden et al. (2020), and our biochemical experiments displayed the C-terminus of Rcf2 crosslinked to Cox12 (IMS protein) and to a C-terminal domain of Cox13 (K85) (section 3.2.1). Due to the crosslinking data, we could also conclude valuable findings about the topology of Rcf3. We were able to verify a crosslink between Rcf3 and Cox13, and according to the mass spectrometry results, it is developed by the $\mathrm{N}$-terminus of Rcf3 and the same C-terminal amino acid of Cox13 (K85) as with Rcf2 (section 3.2.2). Together with the previously mentioned proteinase accessibility assay, this suggests a $\mathrm{N}_{\text {out }}-\mathrm{C}_{\text {out }}$ for Rcf3, as well.

Nevertheless, Zhou et al., (2018a; 2018b; 2020) analyzed Rcf1 and Rcf2 within NMR studies and suggested five transmembrane segments for both proteins. This topology is supported by the assumption that the $\mathrm{C}$-termini are exposed to the mitochondrial matrix. The analyses had been performed in a membrane free environment using detergent micelles and the results are clearly contradictory to all mentioned findings. Thus, we conclude that this is not consistent with the in vivo behavior of Rcf2 and Rcf1. It rather displays a flexible and in fact interesting characteristic of both proteins. They found hydrophilic residues shield their polarity from the lipophilic surface and form so-called "charged zipper" interactions within the lipid layer. By this, they found the proteins homo-dimerize with a second molecule Rcf1/Rcf2 (Zhou et al., 2018b; Zhou et al., 2020). We could not confirm the observation of Rcf1 or Rcf2 dimers with any of our experiments, however, this could be a possibility to interact with other proteins and pictures a responsive nature of Rcf1 and Rcf2.

In the end, our data yielded valuable information about the arrangement of the Rcf-proteins and their residues with favoring the respective termini to stretch into the IMS.

\subsection{Localization of Rcf-proteins}

\subsubsection{Rcf-proteins as substoichiometric interactors of COX}

Different cryo-EM structures did not resolve the Rcf proteins as part of the respiratory supercomplexes (Mileykovskaya et al., 2012; Hartley et al., 2019; Rathore et al., 2019). Rcf1 and Rcf2 were found, however, as part of the corresponding mass spectrometry data (Hartley et al., 2019, 2020). Garlich et al. (2017) characterized Rcf1 as it transiently associates to complex IV and does not remain at the complex. In addition, different populations of complex IV can be detected (Vukotic et al., 2012; Garlich et al., 2017). A considerable amount of Rcf-proteins does 
not reside at the supercomplexes or mature complex IV (Römpler et al., 2016; Dawitz et al., 2020). Co-immunoprecipitation experiments indicate that the Rcf-proteins interact among each other as well (Römpler et al., 2016). Consequently, they were classified as substoichiometric interactors of complex IV and within the supercomplexes $\mathrm{III}_{2} \mathrm{IV}_{(2)}$.

A recently published cryo-EM structure of the yeast respiratory supercomplex, remarkably resolved Rcf2 $2^{107-205}$ as a structural subunit of the hypoxic complex $\operatorname{III}_{2} \mathrm{IV}$ (Hartley et al., 2020). In a previous approach of the same group, a similar density could be identified within the same pocket, formed by Cox3, Cox12 and Cox 13 in complex $\mathrm{III}_{2} \mathrm{IV}_{2}$, but not assigned with certainty (Hartley et al., 2019). They argue that isolated from Cox13 as a late-stage assembled protein, completely matured supercomplexes can be isolated efficiently. Additionally, different experiences proved that the association of Rcf-proteins as peripheral interactors is sensitive to detergent treatment (Vukotic et al., 2012; Römpler et al., 2016). This might be a reason why the proteins were missed in previous structures. Our analyses rather corroborate previous data with the Rcf-proteins, including Rcf2, as substoichiometric interactors of the supercomplex. The endogenous proteins were detected in a wild-type situation to localize at the supercomplexes, yet, the majority of the proteins migrate in smaller complexes (section 3.5.1). These findings deliver circumstantial evidence for a population of supercomplexes being present without Rcf-proteins.

In order to address this question, one could ideally isolate from proteins assembling at a different stage or even the Rcf-proteins themselves and subject the sample to BN-PAGE. The proteins would have to be tagged that native application is possible and isolation efficiency remains comparable. When working on this approach we repeatedly faced the problem that the proteins were not fully functional and caused problems in vivo in biogenesis of the respiratory chain. Consequently, some adjustments would be necessary and functionality can be controlled with the respective RCF mutants as they display impaired growth on non-fermentable media (section 3.1.2).

\subsubsection{The interaction network of Rcf2 and Rcf3}

Chemical crosslinking with subsequent mass-spectrometry analysis revealed Rcf2, Rcf3 and a so far uncharacterized protein Min8 (to be discussed in section 4.1) crosslinked to the cytochrome coxidase (Linden et al., 2020). We could prove these crosslinks to be specific in our biochemical analyses (section 3.2.1 and 3.2.2). It places all three proteins at the periphery of complex IV in contact with Cox12 and, in the case of Rcf2, with Cox13 as well. When evaluating 
our biochemical analysis of Rcf2 crosslinks we could also detect the C-terminal fragment of Rcf2. Our data indicate that the fragment remains at the same localization as full-length Rcf2 as similar specific crosslinks occurred but shifted by the size of cleaved Rcf2 ${ }^{\mathrm{N}}$ (section 3.2.1). This finding furthermore matches the resolved C-terminus of Rcf2 in the cryo-structure of Hartley et al. (2020). The reason that the N-terminus is not resolved could be indicative for partial processing of Rcf2 leading to a heterogeneity in supercomplex populations. In contrast to Rcf2 and Rcf3, crosslinks with Rcf1 could not be detected in the approach we used with crosslinking whole mitochondria (Linden et al., 2020). Due to the high amounts of mitochondrial protein crosslinks, low abundant proteins with transient interactions are hard to detect. Additionally, the topology and transmembrane spans are playing a role since the residues embedded into the membrane are barely accessible for the crosslinker (Linden et al., 2020). An adaption of the analysis as described below could still resolve the interaction with Rcf1 and complex IV by a crosslinking-mass spectrometry approach.

The crosslinking results themselves cannot conclude any timeframe the interaction takes place, being obtained from whole mitochondria. However, it is likely that the crosslink occurs at the supercomplex level, since Rcf2, Rcf3 and Min8 were found in previous mass-spectrometry data collected from supercomplex isolation via Cor1 ${ }^{\text {TAP }}$ (Vukotic et al., 2012). Rcf1 and Rcf2 were also found in mass-spectrometry data of supercomplex isolation via Cox $13^{\text {His }}$. Therefore, it would be interesting if Rcf3 and Min8 were present as well (Hartley et al., 2019, 2020). Unfortunately, the data was only partly published in this case.

In order to get a better understanding of the involvement of the Rcf-proteins and Min8 it could also be a feasible approach to use the Cor1 ${ }^{\text {TAP }}$ isolated supercomplex for a more structural analysis. Since the proteins can be co-isolated, a subsequent crosslinking and mass-spectrometry analysis could identify the interaction on the level of the supercomplex. It could also give more information about the stoichiometry of the proteins. By this, however, one would have to rely on a stable interaction for the isolation beforehand. The advantage of our approach (Linden $e t$ al. 2020) is that the crosslinking procedure with whole mitochondria is performed without the effects of detergents. Subsequently, harsher detergents like SDS can be used for solubilization without disrupting the chemical connection the proteins established via the crosslinker. Thus, one could use this idea the other way around: First, whole mitochondria are subjected to chemical crosslinking and afterwards isolated via Cor1 ${ }^{\text {TAP }}$ to run the mass-spectrometry analysis. Even transient interactions with the supercomplexes would be enriched and a close to in vivo situation could be evaluated. 


\subsection{Min8 - a novel oxidase associated protein}

\subsubsection{Interaction with COX - a new complex IV subunit?}

Chemical crosslinking together with mass spectrometric analysis revealed Min8 as a so far uncharacterized interactor of cytochrome $c$ oxidase (Linden et al., 2020). Vukotic et al. (2012) could identify the small single-pass membrane protein in mass spectrometry data when isolating supercomplexes. Yet, the assignment to complex IV was not addressed. The identified interaction with Cox12 indicates that Min8 is assembled at a late stage of complex IV or supercomplex maturation at the periphery of the complex. The proposed localization of Min8 (Linden et al., 2020) furthermore corresponds with the structure of supercomplex resolved by cryo-EM with Rcf2 being present (Figure 4-1A and B) (Hartley et al., 2020).

We first considered a similarity to the Rcf-protein family but our experiments implied that this is not the case (section 3.3.1). We could prove that Min8 is not essential for respiration and complex IV activity. Our radioactive import experiments however, showed that Cox13 and Cox12 assembly was specifically influenced by MIN8 deletion (section 3.3.2). While Cox13 assembly was more efficient, Cox12 assembly appeared to be compromised. This suggests a specific, although non-essential, role for Min8 in complex IV biogenesis. Interestingly, we found that the topology of Min8 (Linden et al., 2020) resembles the recently identified single spanning IMM protein Cox26. While proven as a structural subunit of complex IV (Hartley et al., 2019), its absence only mildly affects respiration or catalytic function of cytochrome $c$ oxidase. At the same time, biogenesis of complex IV is compromised but supercomplex assembly is not disrupted (Levchenko et al., 2016; Strecker et al., 2016). Consequently, Min8 might represent a structural subunit of complex IV supporting complex stability, similar to Cox26.

Investigations of the human complex IV associated protein NDUFA4/COXFA4 furthermore revealed a partial homology between the transmembrane span of Min8 and NDUFA4 (Balsa et al., 2012). Even more intriguing in this context is the discovery of NDUFA4 within the cryo-EM structure of complex IV, identifying the protein as a structural subunit of cytochrome $c$ oxidase (Zong et al., 2018). Moreover, the interacting site of NDUFA4, resolved in this structure, resembles notably the suggested association of Min8 with yeast cytochrome $c$ oxidase (Figure 4-1B and C) (Zong et al., 2018; Linden et al., 2020). In light of these findings, we propose Min8 as a structural subunit of cytochrome $c$ oxidase. To evaluate this hypothesis, future biochemical experiments are necessary. 
A
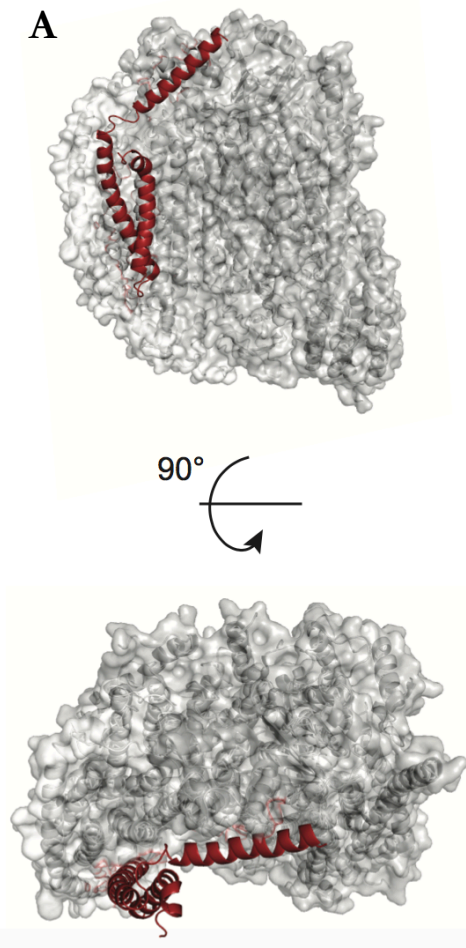

B
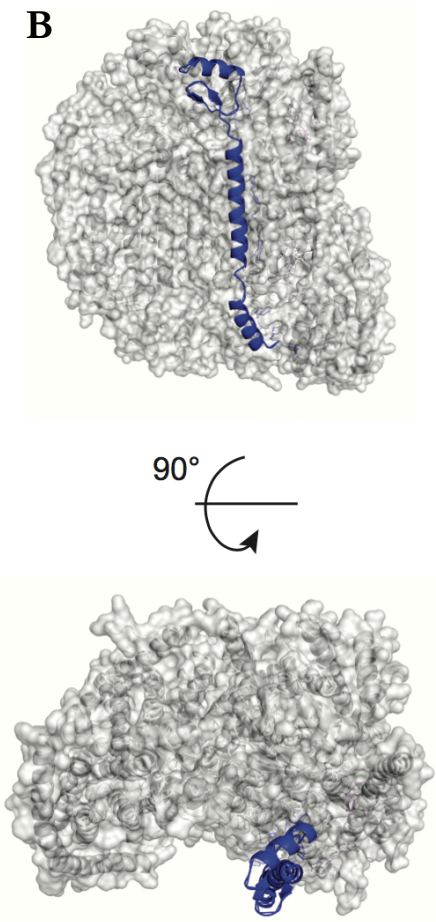

C
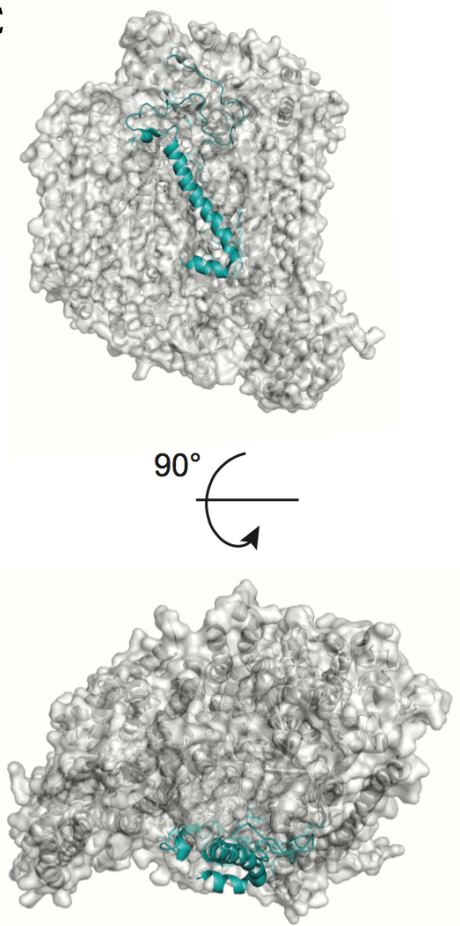

Figure 4-1: Comparing resolved and modeled structures of yeast Rcf2, Min8 and human NDUFA4.

A) Resolved structure of yeast cytochrome $c$ oxidase with Rcf2 (red) extracted from PDB file 6T15 (Hartley et al., 2020).

B) Resolved structure of yeast cytochrome coxidase extracted from PDB file 6HU9 (Hartley et al., 2019) with modeled structure of Min8 (dark blue) from Linden et al. (2020). C) Resolved structure of human cytochrome $c$ oxidase with NDUFA4 (cyan), (PDB file 5Z62; Zong et al., 2018). Editing was performed with the Pymol Software.

\subsubsection{Role in Cox12 assembly}

Given the background of the influence of Min8 on cytochrome $c$ oxidase biogenesis, we discovered a puzzling Cox12 assembly stage, resolved under DDM conditions (3.3.2). It is migrating faster than mature cytochrome $c$ oxidase and still detectable in cox4 $\Delta$ mitochondria. Cox4 represents a structural subunit of complex IV and its deletion causes the absence of mature cytochrome $c$ oxidase (Mick et al., 2007; Vukotic et al., 2012; Su et al. 2014).

Considering Cox12 as a late-stage assembling protein (Mick, Fox and Rehling, 2011), it was surprising that a Cox 12 containing complex is still detectable even when Cox 4 is absent (section 3.3.2). It appears that Cox 12 assembles first into an intermediate complex, which occurs earlier than Cox 4 assembly. However, it is completely unexpected that Cox12 is not detectable in mature complex IV, in wild-type and min $8 \Delta$ mitochondria. This indicates that Cox 12 as an IMS protein dissociates easily from the complex and cannot be observed at mature cytochrome $c$ oxidase under DDM solubilizing conditions. A sensitivity towards DDM had been determined in investigations of the mammalian homologue COX6B as well (Weishaupt and Kadenbach, 
1992; Lazarou et al., 2009). Lazarou et al. (2009) even conducted similar import studies but with mitochondria from human fibroblasts. They obtained a clearly weaker radioactive signal in DDM compared to digitonin solubilized mitochondria after import. Despite this correlation, they found Cox12 also assembled at mature complex IV and an intermediate state was not detected (Lazarou et al., 2009).

Still, the question remained: which proteins are comprised in this newly observed intermediate state? One could imagine that this complex represents an intermediate import state of Cox12. Cox12 as an IMS protein harbors a specific cysteine motif and is imported via Mia40 (Chacinska et al., 2004; Vögtle et al., 2012). Comparing with studies of the MIA pathway with import of other Mia40 substrates (Chacinska et al., 2004), the observed MIA complex resembles remarkably our detected complex. Moreover, Cox12 is assumed to play a role in copper transfer to Cox2. Although this is not completely resolved, it is suggested that Cox12 acts together with the copper chaperones Coa6 and Sco1 on $\mathrm{Cu}_{\mathrm{A}}$ integration into Cox2 (Ghosh et al., 2016). Furthermore, it is proposed that the COX2 module can assemble independently from COX3 and COX1 modules (Franco et al., 2018). Thus, the complex we detect could be a COX2 module maturating state. And the accumulation of radiolabeled Cox12 complex in cox $4 \Delta$ could be justified in this model. Both discussed scenarios involve the cysteine motif which leads to a more stable interaction of Cox12 than in assembled cytochrome $c$ oxidase. This in turn could explain the nature of our detection and that Cox12 cannot be displayed at mature complex IV.

In the end, Min8 shows a specific influence in Cox12 assembly, independent of the composition of the obtained complex. Yet, it would be interesting which proteins are part of this complex. This could be addressed by an adjustment of Cox12 import studies: an antibody shift assay after radiolabeled import could give information about an involvement of Mia40, the copper chaperones and Cox 2 for example. To this end, the import sample would be incubated with specific sera against the proposed interacting proteins. When the addressed protein is present in this association, the detected complex would be shifted considerably. In our attempts, however, this displayed a special challenge because the radioactive signal is weak and hard to detect against the background (data not shown). Thus, different optimization steps would be required to improve import efficiency. Additionally, $C O A G$ mutant mitochondria could be utilized for testing the copper chaperone theory - whereas an import intermediate with Mia40 would remain unaffected. 
Nevertheless, this sort of search for potential Cox12 interactors in this complex is biased and only suspected proteins would be part of it. It would be advantageous to work with a screening approach with mass spectrometry analysis. The challenge in this case, would be the enrichment of that specific complex. This could be accomplished by tagging Cox12 and native co-immunoprecipitation followed by $\mathrm{BN}$-analysis and complexome investigations. However, special mindfulness is required in regard of in vivo behavior of a tagged Cox12 version. This can be examined by phenotype rescue studies, since $\operatorname{cox} 12 \Delta$ is characterized by impaired growth on non-fermentable media (LaMarche et al., 1992).

\subsection{Fusion proteins as a tool to study Rcf-functions}

The Rcf protein family is interconnected through their homologous domains and their interaction network regarding the respiratory supercomplexes. A reported processing event splits Rcf2 and results in two fragments resembling even more Rcf1 and Rcf3 (Römpler et al., 2016). Consequently, we were curious if we could mimic functions by fusing and swapping the respective domains. By this, we intended to get insight into the distinct functional domains. Surprisingly, all three constructs Rcf3-Rcf1, Rcf2 ${ }^{\mathrm{N}}-$ Rcf1 and Rcf3-Rcf2 ${ }^{\mathrm{C}}$ appeared to be functional. This was assessed by different growth drop tests, since $r c f 1 \Delta$ and $r c f 2 \Delta r f 3 \Delta$ display a severe phenotype regarding proper respiration on non-fermentable media (sections 3.1.2, 3.4.2). Thus, these strains represent a useful tool as a transformation background for such functionality tests. It was even more surprising considering that other approaches of tagging the proteins often interfered with proper protein functioning (data not shown). Even the impaired supercomplex formation and Cox13 assembly in ref1 $\Delta$ mitochondria could be complemented by expression of the Rcf1 containing constructs (sections 3.4.4, 3.4.5). In the case for Rcf3-Rcf2 ${ }^{\mathrm{C}}$ it is difficult to ascribe the functionality to either one of the parts or the overall protein. On the one side, $\operatorname{Rcf} 2^{\mathrm{C}}$ alone is not functional (section 3.4.6) and the fusion construct is co-localizing specifically at supercomplexes (section 3.4.2). This differs from endogenous Rcf3. On the other side, we cannot distinguish if the protein forms two sub-populations. One, acting as Rcf3 and the other acting as Rcf2. In the end, this sets the limit to our investigations: The fusion proteins could act as one single protein or as two proteins at different subsets of complexes which we cannot resolve by this approach.

Overall, it was intriguing that the fusion proteins were all functional regarding the respiration. Although we could not directly conclude a specificity for the particular domains we could prove the flexible and diverse involvement of the Rcf-proteins in respiratory function, furthermore discussed below. 


\subsection{Rcf-proteins - genuine respiratory supercomplex factors?}

The Rcf-proteins were first characterized as supercomplex assembly factors - all three proteins are able to interact with complex III and complex IV independently. This unique feature placed them intuitively at the interface of both complexes. Recent findings and our results from this study provide another understanding. Rcf3 and Rcf2 can be placed more peripherally at the cytochrome $c$ oxidase (section 4.2.2 and Linden et al., 2020) and Rcf1 seems to be involved in earlier steps of cytochrome $c$ oxidase assembly (sections 3.1.3, 3.4 .8 and Strogolova et al., 2012, 2019; Garlich et al., 2017; Dawitz et al., 2020).

\subsubsection{Rcf1 - Dual role in supercomplex assembly}

Rcf1 was shown to interact post-translationally with Cox3 as it could be co-purified subsequently to translation assays performed in isolated mitochondria (Strogolova et al., 2012; Su et al., 2014; Garlich et al., 2017). Consequently, the rearrangement of the respiratory chain supercomplexes was ascribed to rather be a cytochrome $c$ oxidase assembly defect than a supercomplex assembly defect itself. Supercomplex assembly is not completely abolished when Rcf1 is absent, however, the presence of the protein facilitates the maturation significantly (section 3.1.2, and Chen et al., 2012; Vukotic et al., 2012; Su et al., 2014; Dawitz et al., 2020).

The early model of the yeast supercomplex envisioned, similarly to mammalian respirasome (Althoff et al., 2011; Dudkina et al., 2011), complex IV interacting with complex III via its convex side, represented by Cox13 (Heinemeyer et al., 2007). The observed association of Rcf1 with Cox13 (Vukotic et al., 2012) furthermore indicated that Rcf1 could act as a proteinaceous link between the complexes. It was shown that Rcf1 plays a role in Cox13 assembly and facilitating Rcf2 assembly (Vukotic et al., 2012). These steps take place in late stage of complex IV/supercomplex assembly. In parallel, Rcf1 still resides at the supercomplexes (Vukotic et al., 2012), which we could also confirm in this work (3.4.2, 3.5.1).

The later obtained cryo-structure of yeast supercomplex of Mileykovskaya et al. (2012) however, provided strong evidence that complex IV orientation differs from mammalian complex IV in the respirasome and complex IV interacts with the opposite side via Cox $5 \mathrm{a} / \mathrm{b}$ with complex III. Consequently, the first anticipated interaction at the interface was discarded. Nevertheless, several studies pointed towards an influence of Rcf1 on catalytic activity of the cytochrome $c$ oxidase by mediating effective cytochrome $c$ binding and slowing down oxygen reduction when deleted (Rydström Lundin et al., 2016; Rydström Lundin and Brzezinski, 2017; Schäfer et al., 2018). This implied an interaction near the cytochrome $c$ binding center (Cox2), again allowing to consider Rcf1 localizing at the now proven interface of complex IV and complex III. 
Due to the evidence for Cox3 and Cox13 interaction, recent publications pictured Rcf1 possibly interacting (similarly to Rcf2, see section 4.2.2) at the periphery of complex IV, opposing the associating side towards complex III (Strogolova et al., 2019; Dawitz et al., 2020). The interaction of Rcf1 with Cox3 is suggested as promoting complex IV integrity by supporting phospholipid integration into Cox3 (Garlich et al., 2017; Hoang et al., 2019; Strogolova et al., 2019). Consequently, the above-mentioned influence on the active site of Cox1 could be mediated indirectly through a different phospholipid environment of Cox3. It was reported that mutating the lipid binding sites of Cox3 in Rhodobacter sphaeroides has a similar effect as if Cox3 was completely absent. The integrated phospholipids influence the stable interaction with Cox1 and protect its active site (Svensson-Ek et al., 2002; Varanasi et al., 2006).

This study's analysis of the fusion proteins shows a specific association of Rcf1 with newly synthesized Cox3 but also Cox2 (section 3.4.8). This speaks not only for an involvement in COX3 modular assembly but also in COX maturation. And by this, arguing for the mentioned indirect influence on complex IV activity. Rcf2 ${ }^{\mathrm{N}}$-Rcf1 and Rcf3-Rcf1 promoted that interaction and simultaneously facilitated supercomplex and Cox13 assembly, yet to a different extent. Rcf2 ${ }^{\mathrm{N}}$-Rcf1 enhanced the interaction of Rcf1 with Cox3 the most (section 3.4.8), but supercomplex- and Cox13 co-localization was less pronounced than with the other fusion proteins (sections 3.4.3 and 3.4.4). This allows the assumption that a tight interaction of Rcf1 with Cox3 does not necessarily generate a favorable basis for supercomplex or Cox13 assembly. This could indicate an involvement of Rcf1 on two different stages with a tight regulation of its presence, first COX assembly and second, supercomplex assembly (Figure 4-2). Similar results are shown in recent studies about the human homolog HIGD2A. There, it was reported that HIGD2A plays first a role in early Cox3 biogenesis, integration of COX3 module in cytochrome c oxidase and assembly of complex IV into the supercomplex (Hock et al., 2020; Timón-Gómez, Garlich, et al., 2020b).

Interestingly, the small protein Coi1 was identified similarly being involved in two so far distinct stages: supercomplex assembly but also Cox1 biogenesis (Singhal et al., 2017). Although a drop of supercomplex assembly in the COI1 deletion mutant could be ascribed to the altered heme integration within Cox1, the study provided strong evidence that Coil is additionally involved at the level of supercomplexes (Singhal et al., 2017). They found not only complex III components as part of the Coi1-complex isolation but also the Rcf-proteins, indicating that the proteins act on the same stage (Singhal et al., 2017). 
These observed interactions of Rcf1 but also Coil on two stages could be accomplished in a model where complex IV assembly occurs simultaneously to supercomplex assembly (Figure 4-2). This is supported by different experienced and published observations: When radiolabeled Cox13 is imported into energized mitochondria, it appears to almost directly assemble into the respiratory supercomplexes (Brandner et al., 2005; Mick et al., 2007; Vukotic et al., 2012). And in fact, recent investigations promote a cooperative complex IV maturation at the site of complex III. Protasoni et al. (2020) revealed an essential role of complex $\mathrm{III}_{2}$ as a structural unit for complex IV maturation in the mammalian system.

The different modules COX1, COX2 and COX3 were found to assemble partly separate from each other, but also in interaction (for a more detailed description see section 1.4.3.). In the imagined model, the COX1 module would represent the initiator of complex IV but also supercomplex assembly, being the first module to interact with complex $\mathrm{III}_{2}$. It was suggested that Cox1 is undergoing less frequent turnover since fewer amounts of newly synthesized Cox1 was found to be present within mature complex IV, compared to Cox2 and Cox3 (McStay, Su and Tzagoloff, 2013). This could be reasoned on the one hand that Cox1 maturation takes additional intermediate states for heme and copper integration and consequently a longer period of time. Or it means Cox1 survives complex IV degradation and waits for newly synthesized Cox 2 and Cox3 and structural subunits to be assembled again (McStay, Su and Tzagoloff, 2013). Our labeling experiments in isolated mitochondria with subsequent isolation of Rcf1-complexes displayed exactly this behavior. Although labeled Cox 2 and Cox 3 could be co-purified together with Rcf1, labeled Cox1 was barely detectable in the bound fraction. Yet, western blot analysis determined that Cox1 was present, speaking for maturated complex IV but with exclusively Cox2 and Cox3 being newly translated (section 3.4.8).

Our model envisions furthermore that COX2 module could either directly interact with COX1 or with COX3 already assembled with COX1. Patient fibroblasts with Cox3 deficiency displayed an association of COX2 and COX1 (Tiranti et al., 2000) while yeast cox2 mutants showed another intermediate: COX3 associating with COX1 in a COX2 independent manner (Horan et al., 2005). This is the probable first association of Rcf1 according to our above-mentioned findings (Figure 4-2, path a). The stabilization of associated COX1 at complex $\mathrm{III}_{2}$ occurs through interacting Rcf1 at COX3 by adequate lipid integration. Therefore, we postulate Rcf1 still as a supercomplex assembly factor. 


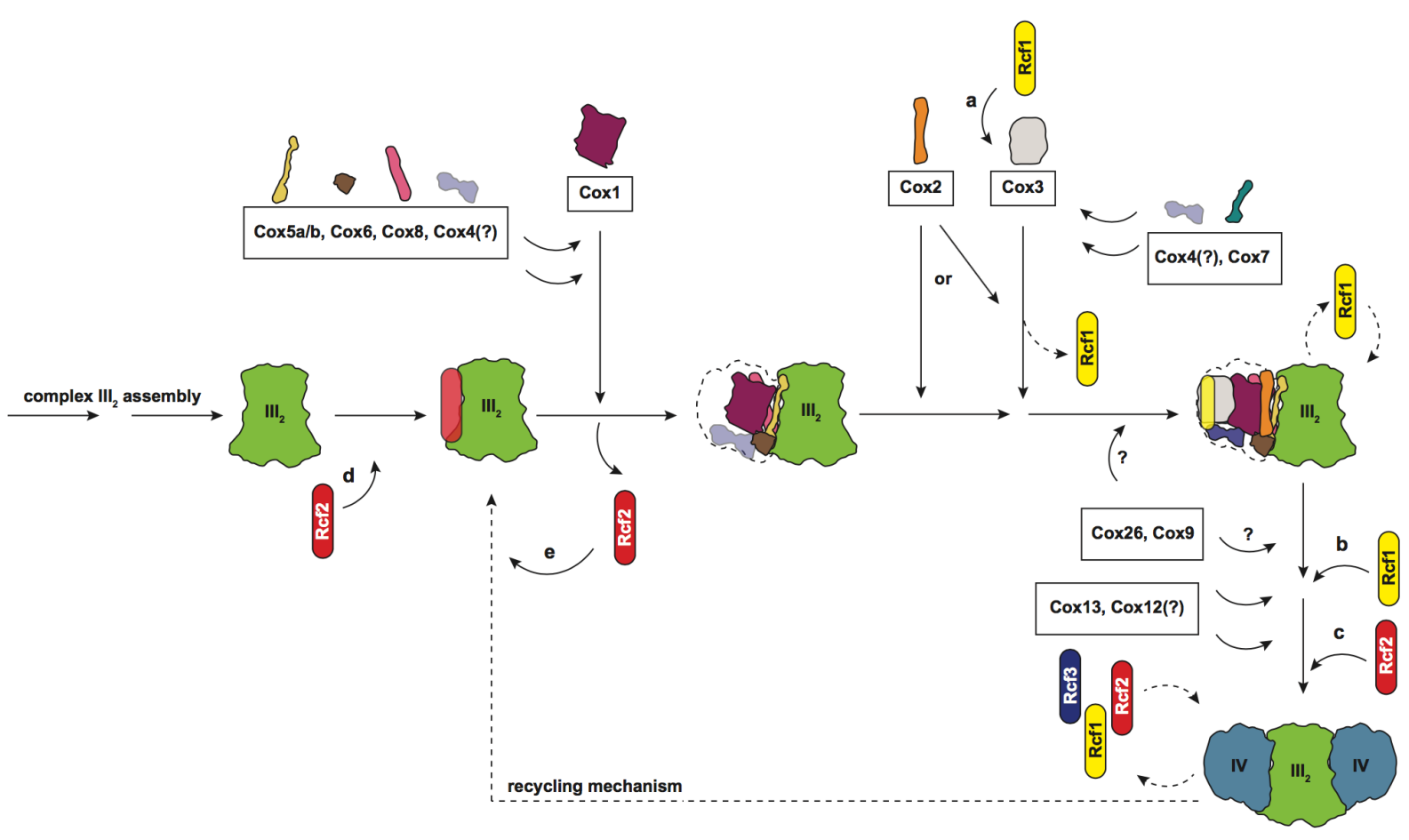

Figure 4-2: Model of Rcf-protein involvement with complex IV and supercomplex assembly.

COX1 module with Cox $5 \mathrm{a} / \mathrm{b}$, Cox6, Cox 8 and probably Cox 4 are interacting directly with complex $\mathrm{III}_{2}$. COX2 either directly interacts with COX1, or enters the assembly pathway after COX3 with Rcf1 as an assembly factor (a). COX3 incorporates Cox7 and in some cases also Cox4. Rcf1 remains at maturating complex IV or dissociates. As facilitating Cox13 and Cox12 assembly, Rcf1 is recruited again (b) for late stage assembly of complex IV. It is not resolved at which stage Cox26 and Cox9 enter complex IV biogenesis. Rcf1 dissociates from fully assembled complex $\mathrm{II}_{2} \mathrm{IV}_{2}$ but can be recruited under certain conditions, i.e. complex IV activity modulation. Rcf2 is recruited to the supercomplex after Rcf1, Cox13 and supposedly Cox 12 assembly (c). Rcf2 and/or Rcf3 alternate with Rcf1 for complex IV regulation. Rcf2 interacts with complex $\mathrm{III}_{2}$ under certain conditions (d), possibly facilitating complex IV assembly and dissociates during further complex IV/supercomplex assembly. The other scenario is, that Rcf2 associates with complex $\mathrm{III}_{2}$ upon disassembly of the supercomplex (e) and demonstrates sort of a recycling mechanism for re-assembly of complex $\mathrm{III}_{2}$ with complex IV. The sequence of events is not resolved and the model does not claim completeness.

Upon finalized supercomplex assembly, it seems that Rcf1 is not present in all supercomplex populations and interacts with the complex depending on its needs such as complex IV activity upregulation. Certainly, Rcf1 is again present at the site of assembled complex IV for efficient Cox13 assembly (Figure 4-2, path b). In the end, the stages where Rcf1 is active are not explicit and rather indistinct, supporting the high dynamics of the respiratory complexes.

To deliver more evidence for this model, however, additional analyses are required. One could be the already mentioned approach with isolating from different stages of assembled supercomplex/cytochrome $c$ oxidase (see section 4.2.1). In addition, import experiments with radioactively labeled Rcf1 combined with different chase timepoints could give more information about the fate and intermediate states of the protein during complex IV and supercomplex biogenesis. 


\subsubsection{Rcf2 - regulation and assembly factor}

Together with previous studies our investigations localize Rcf2 specifically at the periphery of complex IV (see also section 4.2.2). Rcf1 facilitates Cox13 assembly and Rcf2 assembly. Cox13 in turn, influences Rcf2 assembly (Vukotic et al., 2012). Consequently, the sequence of assembly is imagined as Rcf1, Cox13 and then Rcf2 (Figure 4-2, path c).

Our translation assay with following Rcf2 co-immunoprecipitation displayed an interaction with de novo translated Cox3 and Cox2 (section 3.1.3) indicating an earlier timepoint in cytochrome $c$ oxidase association than anticipated. Again, this could support the theory of concurrent complex IV and supercomplex assembly, as mentioned above (4.3.1).

Precedent investigations displayed Rcf2 as a regulatory factor for complex IV activity. When Rcf2 is not present, complex IV activity and respiration are upregulated, while double mutation with Rcf3 lead to a specific drop of complex IV activity (Römpler et al., 2016). And by this, representing a counterpart to Rcf1. Since both proteins are supposed to interact at the same site at Cox3, one could imagine that the proteins' association alternates depending on the required conditions (Figure 4-2). In addition, a recent study of Hoang and colleagues (2019) indicated a role for Rcf2 to prevent a proton back leak through complex IV. This is accompanied with a decreased membrane potential when RCF2 is deleted (Hoang et al., 2019; Strogolova et al., 2019). On the contrary, other studies did not experience a drastic change in membrane potential: Import studies of Cox13 - which is strictly membrane potential dependent - by Vukotic et al. (2012) did not represent a decrease in membrane potential, as well. However, these data rather supported the idea of Rcf2 as a regulatory factor of complex IV and the respiratory supercomplex.

Remarkably, we could identify Rcf2 specifically interacting with complex $\mathrm{III}_{2}$ in a cox5a $\Delta$ situation (section 3.5.1). In light of the introduced model in section 4.3.1, this might indicate a specific role of Rcf2 in assembly of cytochrome $c$ oxidase adjoined to complex $\mathrm{III}_{2}$. Deleting COX $5 A$ creates a special environment: on the one side disturbing COX1 modular assembly by deleting or decreasing an early associated protein. And on the other side generating cytochrome $c$ oxidase exclusively with the other, hypoxic isoform - Cox $5 \mathrm{~b}$ which is expressed in a lesser extent under normoxic conditions (section 3.5.1). Supercomplex assembly is compromised due to the low amounts of cytochrome $c$ oxidase, however, complex $\mathrm{III}_{2} \mathrm{IV}$ is built by incorporating Cox $5 \mathrm{~b}$. This supercomplex basically represents the hypoxic arrangement, although the culture is grown under normal oxygen supply. Consequently, association of Rcf2 
at $\mathrm{III}_{2}$ additionally to $\mathrm{III}_{2} \mathrm{IV}$ can be caused by three different reasons: One, the protein is recruited to the complex specifically under hypoxic conditions (see also section 4.4.2). Two, the situation we detect is visible because of an arrest of complex IV maturation due to delayed assembly (Figure 4-2, path d). And three, our results represent a degradation process with Rcf2 still present or recruited to the dimer after complex IV dissociates from the supercomplex (Figure 4-2 path e). This could represent a sort of a recycling mechanism and reassembly of the supercomplex. Although Rcf2 is proven to not being essential for supercomplex assembly (Chen et al., 2012; Strogolova et al., 2012; Vukotic et al., 2012), this observation represents an exclusive and unique feature, in either of the pictured scenarios.

However, it is hard to imagine that Rcf2 is acting on complex III and complex IV on opposing sites at the same time. It rather speaks for different detected endpoints, the flexible nature of Rcf2 and the dynamics of supercomplex assembly. Since it is prone to interact with complex IV (Vukotic et al., 2012; Römpler et al., 2016), our data indicate that Rcf2 is involved in complex IV assembly at the site of complex III.

\subsubsection{Role of transmembrane spans}

It was intriguing that the N-terminal part of Rcf2 (aa1-83) fused to Rcf1 possesses enough function to rescue the double mutant's phenotype (3.4.2). The HIG1 domain stretches from aa89 to 180 and, in the case of Rcf2 ${ }^{\mathrm{N}}$-Rcf1, is only present in Rcf1. At the same time, the fragment itself is not capable of functioning (section 3.4.6) and it appears that the overall Rcf2like topology supports proper assignment. It could also indicate that the HIG1 domain or the rather polar residues (described in section 4.1) are crucial for guiding the protein to the required localization and the protein fulfills the function through its topological characteristics. Still, this might not be at the level of supercomplexes since we could not detect Rcf $2^{\mathrm{N}}$-Rcf1 as strongly associated there (sections 3.4.2, 3.5.2.). Another reason for functionality of Rcf $2^{\mathrm{N}}-\mathrm{Rcf} 1$ or Rcf3-Rcf2 ${ }^{\mathrm{C}}$ in contrast to the single Rcf2 fragments could be that the dedicated import pathway might be impaired and the fragments are not folded properly into the IMM. Rcf-proteins do not harbor a presequence and it is not completely resolved, which import route the Rcf-proteins take. Rcf2 was found to be depleted upon OXA1 COX18 double deletion (Stiller et al., 2016). This argues for Rcf2 undergoing the conservative sorting pathway comprising concerted action of TIM23 and Oxa1 (see also section 1.3.2) (Römpler, 2016; dissertation). Oxa1 dependent export might rely on the presence of specific transmembrane spans which would be disrupted when several domains are absent. 
Although we could determine the functionality of Rcf $2^{\mathrm{N}}$-Rcf1 as Rcf2-like in terms of respiration capacity, we found the strains expressing Rcf2 ${ }^{\mathrm{N}}-\mathrm{Rcf1}$ experienced elevated ROS levels (section 3.4.7). This points towards enhanced stressing conditions, yet with unclear reason. When the internal processing event fulfills the function of regulating the presence of $\mathrm{Rcf2}^{\mathrm{N}}$, it might be disturbed when Rcf1 is fused to the fragment and leads to interference with endogenous Rcf2 action. Since Rcf2 ${ }^{\mathrm{N}}$ stretches until aa83 in our construct, the processing site is likely to be still present; it was proposed to occur at aa63 (Römpler et al., 2016). In fact, different experiments also led to the detection of processed versions of the fusion protein, still detectable with the antibody against Rcf1 (data not shown). Although it would be interesting to follow the idea of affected processing, it is difficult to directly compare endogenous Rcf2 processing with Rcf2 ${ }^{\mathrm{N}}$-Rcf1 since both products would be detected with different antibodies. Assuming that Rcf2 ${ }^{\mathrm{N}}-\mathrm{Rcf1}$ is processed at least partially at the endogenous Rcf2 processing site, this could indicate the importance of overall similar topology for proper processing of Rcf $2^{\mathrm{N}}$. This would not be ensured when the C-terminus is lacking, representing another explanation for the different capability of the fragment alone (section 3.4.6).

Overall, our results of analyzing Rcf2 ${ }^{\mathrm{N}}$-Rcf1 speak for a distinct function of Rcf2 ${ }^{\mathrm{N}}$ in addition to $\mathrm{Rcf} 2 \mathrm{C}$. It was reported previously that assembly of Rcf2 is directed via the C-terminus. A truncated $\mathrm{N}$-terminus even facilitated assembly into respiratory supercomplexes, leading to the assumption that Rcf2 ${ }^{\mathrm{N}}$ is rather negligible (Römpler et al., 2016). Our understanding of Rcf2 ${ }^{\mathrm{N}}$ in light of the fusion proteins however, disproves this hypothesis.

We were furthermore eager whether the fusion constructs expressed in the COX5A mutant could resolve if certain domains favor the complex $\mathrm{III}_{2}$ association. But none of the constructs showed a co-migration with complex $\mathrm{III}_{2}$ in our $2^{\text {nd }}$ dimension analysis (section 3.5.2). Although this does not allow an attribution to any of the Rcf2 halves, it speaks for a unique role of Rcf2 in this context which was not represented by just fusing its transmembrane spans to Rcf1 or Rcf3.

\subsubsection{Role of internal processing event}

The idea that specific transmembrane spans are responsible for the interaction of Rcf2 with complex $\mathrm{III}_{2}$ in $c 0 \times 5 a \Delta$, implied at the same time that the processing event itself might be essential. We investigated both fragments by adding a FLAG tag N-terminally to Rcf2 as introduced before by Römpler et al. (2016). It could be exposed that $\operatorname{Rcf2}^{\mathrm{C}}$ and ${ }^{\mathrm{FLAG}}{ }^{\mathrm{R}} \mathrm{f} 2{ }^{\mathrm{N}}$, also 
reside at complex $\mathrm{III}_{2} \mathrm{IV}$ and $\mathrm{III}_{2}$ (section 3.5.3). That both fragments are present at the same complexes indicates that they descend from prior bound full-length Rcf2 upon processing. When investigated under wild-type conditions, ${ }^{\mathrm{FLAG}}{ }^{\mathrm{Rcf}} 2^{\mathrm{N}}$ could not be detected at the supercomplexes (Römpler et al., 2016), indicating a labile nature of the fragment and a tight regulation. This seem to be a different situation in our experiments in the cox $5 a \Delta$ background and places again special emphasis on the internal processing event of Rcf2.

Interestingly, both fragments co-migrate in a similar ratio at $\mathrm{III}_{2} \mathrm{IV}$ and $\mathrm{III}_{2}$ as full-length Rcf2 (section 3.5.3). Unfortunately, this does not provide evidence for a sequence of events. One scenario is that processing occurs during or pre supercomplex assembly and facilitates complex IV assembly in an upstream motion (Figure 4-3A). However, only a fraction of Rcf2 is processed. It is likely that Rcf2 dissociates from the complex upon supercomplex assembly and is recruited when required, as described above (section 4.3.2). The second scenario would be that the detected co-migration represents degrading steps and Rcf2 is processed at the supercomplex (Figure 4-3B). The majority of full-length Rcf2 and both fragments is detectable at complex $\mathrm{III}_{2} \mathrm{IV}$ proposing the processing occurs at this stage. In parallel, it remains inconclusive why both fragments and full-length Rcf2 still co-localize at complex $\mathrm{III}_{2}$.

To resolve the sequence of events, one approach could be, observing the interaction of Rcf2 when complex IV maturation is abolished additionally. This could be accomplished by deleting the structural unit Cox4 which assembles later than Cox5a/Cox5b (Mick et al., 2007). Thus, Cox $5 b$ assembly still occurs but a possible downstream pathway from complex $\mathrm{III}_{2} \mathrm{IV}$ could be excluded. However, it would be essential to increase Cox 5 b expression similarly to Cox $5 \mathrm{a}$ under normoxic conditions as described in section 4.4.2, otherwise the cell growth is likely to be heavily compromised. 
A

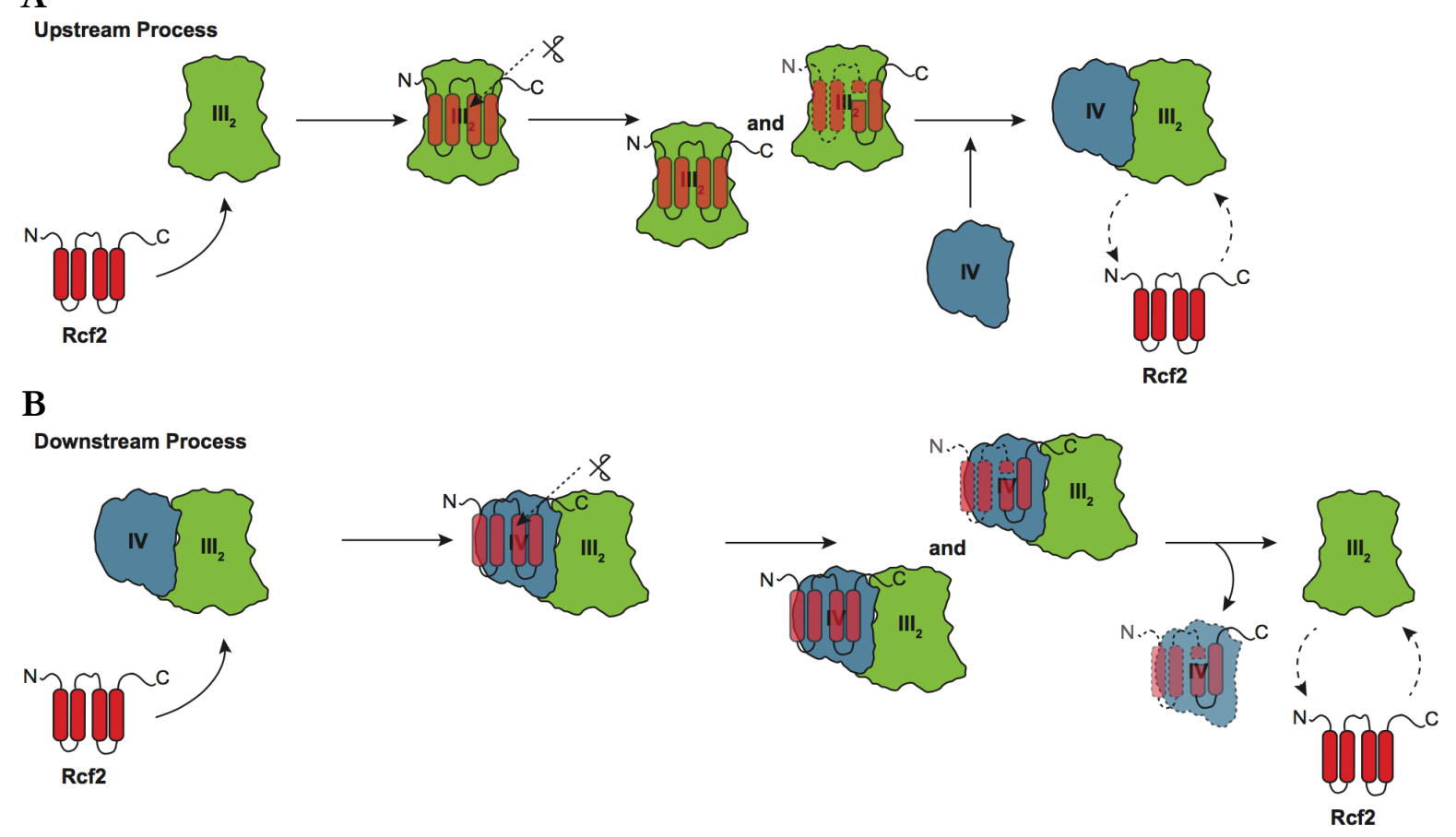

Figure 4-3: Rcf2 processing - model of possible scenarios.

A) Rcf2 processing occurs during or pre- supercomplex assembly at complex $\mathrm{III}_{2}$. Two populations of Rcf2 exist due to partial processing. The dashed lines indicate a fast degradation and lability of Rcf $2^{\mathrm{N}}$. After complete supercomplex assembly, Rcf2 is recruited under certain conditions i.e. complex IV activity modulation B) Rcf2 is processed at the supercomplex during a degradation mechanism of the supercomplex. The dashed lines indicate a fast degradation and instability of Rcf2 ${ }^{\mathrm{N}}$. Rcf2 is still found to interact with complex $\mathrm{III}_{2}$. The model only represents complex $\mathrm{III}_{2} \mathrm{IV}$ for convenience, the processing is envisioned to occur the same with a second complex IV monomer.

Overall, the purpose and triggering conditions of Rcf2 processing are elusive. Especially, since the majority of Rcf2 remains intact. In addition, the interaction site of Rcf2 at complex $\mathrm{III}_{2}$ needs further investigation. To address this question, the biggest challenge would be to dissect complex $\mathrm{III}_{2}$ from $\mathrm{III}_{2} \mathrm{IV}$ but under in vivo conditions. DDM as a detergent is a feasible choice to dissociate complex $\mathrm{III}_{2}$ from complex IV. However, it often failed to solubilize the complexes together with transient interactors such as the Rcf-proteins (Vukotic et al., 2012; Römpler et al., 2016). Consequently, other detergent conditions should be tested in this regard.

In the end, it was not the aim of this study to define the processing site, but it appears as a crucial point in investigating the role of Rcf 2 in the future. The fusion construct could be a useful tool in this regard. With different truncations of Rcf $2^{\mathrm{N}}$ from both sites - still fused to Rcf1, it would be interesting to test if a lack of functionality comes together with truncating the putative processing site. 


\subsubsection{Rcf3 - regulation of supercomplex capacity}

Rcf3 as the smallest protein is also the least characterized of the Rcf-protein family. Römpler et al. (2016) identified it as a regulatory factor of complex IV. However, only the double deletion together with RCF2 demonstrates the substantial relevance for respiration. Complex IV activity is then strongly affected and lead to the inability of proper OXPHOS function without causing an obvious rearrangement of the respiratory chain (section 3.1.2 and Römpler et al., 2016). Our investigations of Rcf3 within the fusion proteins support this understanding but fine-tune it at the same time (section 3.4.7). The fusion proteins harboring Rcf3 were able to overcome the crucial respiratory defect of $r f 2 \Delta r c f 3 \Delta$ (section 3.4.2). In parallel, they hampered the respiration capacity significantly, independent from the presence of endogenous Rcf3 (section 3.4.7). Yet, this effect is not measurable via isolated complex IV activity. The constructs appeared to rather influence respiration on level of the supercomplexes together with electron transfer via complex III, addressed by the other states: LEAK, state 3 and OXPHOS. However, endogenous Rcf3 functions seem to be disturbed considering the detected effect regarding oxygen consumption of Rcf3-Rcf1 and Rcf3-Rcf2 ${ }^{\mathrm{C}}$ in $\operatorname{rcf} 2 \Delta \operatorname{rcf} 3 \Delta$ mitochondria. Since the expression of fusion proteins is not accompanied by rearrangement of the respiratory chain complexes (sections 3.4.2, 3.4.3), the effect does not speak for a substantial structural function of Rcf3. It suggests a regulatory role especially pronounced when Rcf3 is associated with the respiratory supercomplexes. Our results also indicate that presence of Rcf3 at the site of $\mathrm{III}_{2} \mathrm{IV}_{(1-2)}$ is strongly regulated. The fusion to the transmembrane domains of Rcf2 ${ }^{\mathrm{C}}$ or Rcf1 appear to interfere with this process and Rcf3 remains tethered to the complexes. $2^{\text {nd }}$ dimension analysis of mitochondria from wild-type cells expressing the fusion proteins confirms this impression (section 3.4.2). The constructs harboring Rcf3 show an increased localization to the supercomplexes compared to endogenous Rcf3.

We propose that Rcf3 is recruited directly to the supercomplex as a modulating factor for respiration and dissociates upon strict regulation with a so far unknown mechanism (Figure 4-2, section 4.3.1). At the same time, it appears that Rcf3 already interacts with newly translated Cox3 and Cox2 (section 3.1.3). This fact repeatedly supports a model in which supercomplex assembly and cytochrome $c$ oxidase maturation are adaptable mechanisms occurring in parallel rather than separately. 


\subsection{Role of Rcf-proteins under stressing conditions}

\subsubsection{Involvement in oxidative stress}

Several studies connected a putative Rcf-protein function to oxidative stress by protecting from ROS production (Chen et al., 2012; Vukotic et al., 2012; Römpler et al., 2016). Rcf1 and Rcf2 were first characterized to influence ROS production specifically. Vukotic et al. (2012) argued, that complex IV*, which harbors Cox13 and likely Rcf1 and Rcf2, prevents from mitochondrial ROS production while the respective mutants $r f 1 \Delta, r f 2 \Delta$, cox13 $\Delta$ display high ROS levels. Rcf1 $\Delta$ showed the most pronounced phenotype in this regard. This suggested a specific role for Rcf1 in contrast to Rcf2 or Cox13, and could be explained as a result of the rearrangement of respiratory supercomplexes (Vukotic et al., 2012). In recent investigations of supercomplexes and their function, however, they found that ROS production was not significantly higher when supercomplex assembly itself is disturbed (Berndtsson et al., 2020), arguing even more for a specific function of Rcf1. Additionally, cells lacking Rcf1 developed an increased sensibility against $\mathrm{H}_{2} \mathrm{O}_{2}$ supporting the idea of an essential protein during oxidative stress (Chen et al., 2012). Since Rcf2 and Rcf3 were assigned to cover overlapping functions (Römpler et al., 2016), Rcf3 should be included in this consideration.

Our measurements of mitochondrial ROS production not only confirmed previous findings of the RCF1 mutant but also provided new results regarding the RCF2RCF3 double mutant. Isolated mitochondria from $\operatorname{rcf} 2 \Delta \operatorname{rcf} 3 \Delta$ cells displayed elevated ROS levels, although not as high as from $r c f 1 \Delta$ (3.4.7). We did not determine the ROS level of $r c f 2 \Delta$ in these experiments, thus we cannot conclude an effect of additional deletion of RCF3. However, our results in section 3.4.7 imply that the increased ROS production is a secondary effect caused by the respective deletions. Although the fusion constructs fail to lower the ROS level of $\operatorname{rcf} 2 \Delta \operatorname{rcf} 3 \Delta$ and Rcf2 ${ }^{\mathrm{N}}$-Rcf1 induces it even more, the characteristic growth-phenotype of the double mutant is complemented. Thus, the growth-phenotype is presumably not caused by a sensitivity towards the high ROS level. Another phenotype, which is displayed by $\operatorname{rrf} 2 \Delta \operatorname{rcf} 3 \Delta$ is the decreased complex IV activity (section 3.4.7 and Römpler et al., 2016). The fusion constructs were all able to rescue the lowered complex IV activity. Consequently, the growth-phenotype can be ascribed to the effect on complex IV activity.

Interestingly, the fusion protein Rcf $2^{\mathrm{N}}$-Rcf1 expressed in $r f 2 \Delta r c f 3 \Delta$ results in an OXPHOS capacity which exceeds the wild-type capacity over $50 \%$. As mentioned, this strain caused one of the highest ROS levels measured in our studies of the fusion constructs (section 3.4.7). It 
appears intuitive that high respiration might lead to higher ROS levels, yet $r f 1 \Delta$ causes both: low respiration and high ROS levels (Chen et al., 2012; Vukotic et al., 2012).

In the end, our results indicate a unique involvement of the Rcf-protein family not only by preventing from uncontrolled ROS production but also protecting from high ROS levels. Thus, the Rcf-proteins appear to play an important role in oxidative stress mediated by the respiratory chain.

\subsubsection{Influence in adaptation to hypoxia}

Rcf1 and Rcf2 comprise a conserved HIG1 domain. First, they were identified among genes, expressed under hypoxia, which was extended to proteins with the significant motif $(\mathrm{Q} / \mathrm{I}) \mathrm{X}_{3}(\mathrm{R} / \mathrm{H}) \mathrm{XRX}_{3} \mathrm{Q}$ (or QRRQ). The HIG1 domain, in general, can be clustered into two subgroups: HIG1 type 1 and HIG1 type 2 isoforms. While HIG1 type 1 isoforms are only present in higher eukaryotes, HIG1 type 2 isoforms are established in all eukaryotes and $\alpha$-prokaryotes. The latter subgroup includes yeast Rcf1, Rcf2 and mammalian HIGD2A, whereas HIGD1A is classed amongst HIG1 type 2 isoforms (Bedo et al., 2004; Wang et al., 2006; Garlich et al., 2017). Rcf1 and Rcf2 are constitutively expressed, as demonstrated by all our experiments. While it was shown that Rcf1 and Rcf2 are even more required under low oxygen concentrations, a specific upregulation of Rcf1 and Rcf2, mediated by hypoxia, was not reported (Vukotic et al., 2012; Garlich et al., 2017; Strogolova et al., 2019).

Of the two mammalian homologs, HIGD2A is considered to be a true homolog of Rcf1. In complementation assays, HIGD2A was able to rescue Rcf1 mediated yeast phenotype, whereas HIGD1A was not (Vukotic et al., 2012). Studies of HIGD1A and HIGD2A showed that they display a basal expression under normoxia which is significantly induced during early states of hypoxia (Timón-Gómez, Garlich, et al., 2020b). However, another recent study found only HIGD1A expression adapted to hypoxia while HIGD2A remains stably expressed (Hock et al., 2020). In rat cardiomyocytes, Higd1a was reported as an important modulator of complex IV activity, by inducing structural changes in the heme $a$ active center of cytochrome $c$ oxidase. At the same time, Higd1a prevented hypoxic mediated cell death by advancing ATP production (Hayashi et al., 2015).

Given these presented functions of the mammalian homologues, we can also imagine a similar role for yeast Rcf-proteins during adaptation to hypoxia. Rcf proteins can modulate complex IV and supercomplex assembly and capacity, not only in a structural way (see sections above). Already favorable under normoxic conditions, it might play an even more substantial role under hypoxia. 
It is still obscure, how the respiratory chain copes with the switch between the isoforms represented either under normoxia or hypoxia. Especially relevant for the respiratory chain are the isoforms Cyc1/Cyc7 and Cox5a/Cox5b in this context (Hodge et al., 1989; Zitomer, Carrico and Deckert, 1997; Dodia et al., 2014). While both cytochrome $c$ isoforms (Cyc1/Cyc7) are soluble in the IMS and associate with complex III and complex IV for electron transfer, Cox $5 \mathrm{a} /$ Cox $5 \mathrm{~b}$ are integral membrane proteins associated at the interface of complex III and complex IV. The interaction of Cox $5 \mathrm{a} / \mathrm{Cox} 5 \mathrm{~b}$ with Cor1 links the two complexes and proposed as the structural basis for supercomplex formation (Hartley et al., 2019, 2020; Rathore et al., 2019). Consequently, it is likely that the supercomplex dissociates for an exchange. Either the subunit itself is exchanged or mature hypoxic complex IV is incorporated into supercomplexes. Our data from evaluating the $\operatorname{cox} 5 a \Delta$ situation, together with the cryo-EM structure resolving Rcf2 at hypoxic supercomplexes (Hartley et al., 2020), indicate a specific role for Rcf2 during hypoxia. We propose a model, where Rcf2 is either recruited to complex $\mathrm{III}_{2}$ or remains associated at complex $\mathrm{III}_{2}$ after degradation processes in order to facilitate and stabilize hypoxic supercomplex assembly (see also 4.3.2.2).

For further analyses of an involvement of Rcf2 in the hypoxic/normoxic switch of isoforms, it would be essential to upregulate the Cox $5 b$ level, comparable to the Cox5a level in wild-type. This can be either accomplished by working under real hypoxic conditions or by using the approach from Hartley et al. (2020). They expressed COX5B under the promotor of COX5A in the background of $\cos 5 a \Delta$. By this, a hypoxic form of supercomplexes would be generated but a putative state of assembly arrest would be omitted. 



\section{Summary and Conclusion}

Oxidative Phosphorylation as the most prominent mitochondrial function, underlies the mechanism of efficient electron transport to reduce oxygen to water and the coupled proton translocation across the mitochondrial membrane. This is suggested to be one major function of the arrangement of stable respiratory supercomplexes. However, the mechanism by which the single complexes are organized into this higher order structures is still unclear.

This study aimed for a better understanding of supercomplex assembly, its stabilization and the involvement of the Rcf-proteins. During investigating the interactome of the respiratory chain together with Linden et al. (2020), a novel putative subunit of complex IV came into our attention: Min8. Basic characterizations revealed that it is not essential for respiration or supercomplex formation, however, we obtained an involvement in late-stage complex IV assembly. Surprising in this regard was the observation of a supposed complex IV intermediate state of Cox12 containing complex which was not experienced before and appears to be independent from subsequent complex IV maturation.

Within the same collaboration (Linden et al. 2020) we could specifically assign interaction sites of Rcf2 and Rcf3 with Cox12 and Cox13 and validate the method of chemical crosslinking of whole mitochondria together with mass-spectrometry analysis. Cox12 and Cox13 are late-stage assembly proteins of complex IV, possibly recruited directly to the supercomplex. This goes in line with our observation that the interaction platform of the Rcf-proteins with complex IV are predominantly the supercomplexes $\mathrm{III}_{2} \mathrm{IV}_{(1-2)}$. However, it appeared that the encounter with complex IV subunits starts early considering the association with the de novo mitochondrially translated subunits Cox3 and Cox2. In light of the "plasticity" model, where it is suggested that mature holo-complexes and supercomplexes are undergoing constant dynamic dis-/association, our data indicates that this might be taken even a step further. Complex IV maturation could not exclusively happen sequestered from supercomplex assembly but in parallel, and we envision that Rcf1 plays an important role in the assembly and stabilization of both complex IV and the supercomplexes.

Moreover, our data supports the model of the Rcf-proteins being substoichiometric and transient interactors of the supercomplexes and their presence is strictly regulated. Considering the shared homologous sequences, artificial fusion proteins with swapped transmembrane domains were constructed and their impact on Rcf-function was analyzed in detail. We could obtain a very dynamic nature of Rcf-functions and the fusion proteins were able to complement the different phenotypes in a highly flexible manner. However, the tight regulation of 
Rcf-proteins was apparently disturbed and the respective functional domains seemed to be tethered to a certain stage, displayed especially by the construct Rcf2 ${ }^{\mathrm{N}}-\mathrm{Rcf} 1$. Although the protein was able to restore Rcf2 function, it caused high levels of reactive oxygen species, while being retained at complex IV. This speaks not only for distinct roles of Rcf2 ${ }^{\mathrm{N}}$ and $\mathrm{Rcf} 2^{\mathrm{C}}$ but also for a physiological role of Rcf2 processing being compromised under these conditions. Rcf3 was proven once more to be a regulator of complex IV, yet, according to our data especially on supercomplex level with complex III and complex IV working in tight cooperation.

Moreover, we could ascertain a specific interaction of Rcf2 with hypoxic supercomplexes by utilizing the COX5A deletion strain, whereas Rcf1 and Rcf3, normally present as well, seem to dissociate. Rcf2 was determined to interact at complex $\mathrm{III}_{2}$ under the same conditions, which we envision as a transition state descending from, or assembling into hypoxic supercomplexes. It is tempting to imagine Rcf2 as a player within supercomplex reorganization for the subunit exchange due to altering oxygen levels, while intrinsic processing might play a role.

Overall, this work has provided a detailed analysis of Rcf-protein function and put supercomplex assembly and the respective interactors into a new perspective. 


\section{Bibliography}

Abe, Y. et al. (2000) 'Structural Basis of Presequence Recognition by the Mitochondrial Protein Import Receptor Tom20', Cell, 100(5), pp. 551-560. doi: 10.1016/S00928674(00)80691-1.

Acín-Pérez, R. et al. (2004) 'Respiratory Complex III Is Required to Maintain Complex I in Mammalian Mitochondria', Molecular cell, 13(6), pp. 805-815.

Acín-Pérez, R. et al. (2008a) 'Respiratory Active Mitochondrial Supercomplexes', Molecular Cell, 32(4), pp. 529-539. doi: 10.1016/j.molcel.2008.10.021.

Acín-Pérez, R. et al. (2008b) 'Respiratory active mitochondrial supercomplexes', Molecular cell, 32(4), pp. 529-539.

Acín-Pérez, R. and Enriquez, J. A. (2014) 'The function of the respiratory supercomplexes: The plasticity model', Biochimica et Biophysica Acta (BBA) - Bioenergetics, 1837(4), pp. 444-450. doi: 10.1016/j.bbabio.2013.12.009.

Allen, J. F. (2015) 'Why chloroplasts and mitochondria retain their own genomes and genetic systems: Colocation for redox regulation of gene expression', Proceedings of the National Academy of Sciences of the United States of America, 112(33), pp. 1023110238. doi: 10.1073/pnas. 1500012112.

Althoff, T. et al. (2011) 'Arrangement of electron transport chain components in bovine mitochondrial supercomplex I1III2IV1', The EMBO Journal, 30(22), pp. 4652-4664. doi: 10.1038/emboj.2011.324.

Balsa, E. et al. (2012) 'NDUFA4 Is a Subunit of Complex IV of the Mammalian Electron Transport Chain', Cell Metabolism, 16(3), pp. 378-386. doi: 10.1016/j.cmet.2012.07.015.

Bareth, B. et al. (2013) 'The Heme a Synthase Cox15 Associates with Cytochrome c Oxidase Assembly Intermediates during Cox1 Maturation', Molecular and Cellular Biology, 33(20), pp. 4128-4137. doi: 10.1128/MCB.00747-13.

Barrientos, A., Zambrano, A. and Tzagoloff, A. (2004) 'Mss51p and Cox14p jointly regulate mitochondrial Cox $1 \mathrm{p}$ expression in Saccharomyces cerevisiae', The EMBO Journal, 23(17), pp. 3472-3482. doi: 10.1038/sj.emboj.7600358.

Barros, M. H. and McStay, G. P. (2020) 'Modular biogenesis of mitochondrial respiratory complexes', Mitochondrion, 50, pp. 94-114. doi: 10.1016/j.mito.2019.10.008.

Bazán, S. et al. (2013) 'Cardiolipin-dependent Reconstitution of Respiratory Supercomplexes from Purified Saccharomyces cerevisiae Complexes III and IV*,, 
Journal of Biological Chemistry, 288(1), pp. 401-411. doi: 10.1074/jbc.M112.425876.

Bedo, G. et al. (2004) 'Characterization of Hypoxia induced gene 1: expression during rat

Central Nervous System maturation and evidence of antisense RNA expression',

International Journal of Developmental Biology, 49(4), pp. 431-436. doi:

10.1387/ijdb.041901gb.

Benz, R. (1994) 'Permeation of hydrophilic solutes through mitochondrial outer membranes: review on mitochondrial porins', Biochimica et Biophysica Acta (BBA) Reviews on Biomembranes, 1197(2), pp. 167-196. doi: 10.1016/0304-4157(94)90004-3.

Berndtsson, J. et al. (2020) 'Respiratory supercomplexes enhance electron transport by decreasing cytochrome c diffusion distance', EMBO reports, 21(12), p. e51015. doi: 10.15252/embr.202051015.

Blomberg, M. R. A. (2016) 'Mechanism of Oxygen Reduction in Cytochrome c Oxidase and the Role of the Active Site Tyrosine', Biochemistry, 55(3), pp. 489-500. doi: 10.1021/acs.biochem.5b01205.

Bohnert, M. et al. (2010) 'Cooperation of Stop-Transfer and Conservative Sorting Mechanisms in Mitochondrial Protein Transport', Current Biology, 20(13), pp. 12271232. doi: 10.1016/j.cub.2010.05.058.

Böttinger, L. et al. (2012) 'Phosphatidylethanolamine and Cardiolipin Differentially Affect the Stability of Mitochondrial Respiratory Chain Supercomplexes', Journal of Molecular Biology, 423(5), pp. 677-686. doi: 10.1016/j.jmb.2012.09.001.

Bradford, M. M. (1976) 'A rapid and sensitive method for the quantitation of microgram quantities of protein utilizing the principle of protein-dye binding', Analytical Biochemistry, 72(1), pp. 248-254. doi: 10.1016/0003-2697(76)90527-3.

Brand, M. D. (2010) 'The sites and topology of mitochondrial superoxide production', Experimental Gerontology, 45(7-8), pp. 466-472. doi: 10.1016/j.exger.2010.01.003.

Brandner, K. et al. (2005) 'Taz1, an Outer Mitochondrial Membrane Protein, Affects Stability and Assembly of Inner Membrane Protein Complexes: Implications for Barth Syndrome', Molecular Biology of the Cell, 16(11), pp. 5202-5214. doi: 10.1091/mbc.e05-03-0256.

Brix, J. et al. (1999) 'Distribution of Binding Sequences for the Mitochondrial Import Receptors Tom20, Tom22, and Tom70 in a Presequence-carrying Preprotein and a Noncleavable Preprotein*', Journal of Biological Chemistry, 274(23), pp. 16522-16530. doi: $10.1074 / j b c .274 .23 .16522$.

Chacinska, A. et al. (2004) 'Essential role of Mia40 in import and assembly of 
mitochondrial intermembrane space proteins', The EMBO Journal, 23(19), pp. 37353746. doi: 10.1038/sj.emboj.7600389.

Chance, B. and Williams, G. R. (1955) 'A Method for the Localization of Sites for Oxidative Phosphorylation', Nature, 176(4475), pp. 250-254. doi: 10.1038/176250a0.

Chen, Y.-C. et al. (2012) 'Identification of a Protein Mediating Respiratory Supercomplex Stability', Cell Metabolism, 15(3), pp. 348-360. doi: 10.1016/j.cmet.2012.02.006.

Clarke, S. L. et al. (2013) 'Barth syndrome', Orphanet Journal of Rare Diseases, 8(1), p. 23. doi: 10.1186/1750-1172-8-23.

Claude, A. (1946) 'FRACTIONATION OF MAMMALIAN LIVER CELLS BY DIFFERENTIAL CENTRIFUGATION', The Journal of Experimental Medicine, 84(1), pp. 51-59.

Colina-Tenorio, L. et al. (2020) 'Shaping the mitochondrial inner membrane in health and disease', Journal of Internal Medicine, 287(6), pp. 645-664. doi: https://doi.org/10.1111/joim.13031.

Conte, A. et al. (2015) 'The dimerization of the yeast cytochrome bc1 complex is an early event and is independent of Rip1', Biochimica et Biophysica Acta (BBA) - Molecular Cell Research, 1853(5), pp. 987-995. doi: 10.1016/j.bbamcr.2015.02.006.

Costanzo, M. C. and Fox, T. D. (1986) 'Product of Saccharomyces cerevisiae nuclear gene PET494 activates translation of a specific mitochondrial mRNA.', Molecular and Cellular Biology, 6(11), pp. 3694-3703. doi: 10.1128/MCB.6.11.3694.

Cumsky, M. G. et al. (1987) 'Structural analysis of two genes encoding divergent forms of yeast cytochrome c oxidase subunit V.', Molecular and Cellular Biology, 7(10), pp. 3511-3519.

Davies, K. M., Blum, T. B. and Kühlbrandt, W. (2018) 'Conserved in situ arrangement of complex I and III 2 in mitochondrial respiratory chain supercomplexes of mammals, yeast, and plants', Proceedings of the National Academy of Sciences, 115(12), pp. 3024-3029. doi: 10.1073/pnas.1720702115.

Davoudi, M. et al. (2016) 'COX7A2L/SCAFI and Pre-Complex III Modify Respiratory Chain Supercomplex Formation in Different Mouse Strains with a Bcs11 Mutation', PLoS ONE, 11(12). doi: 10.1371/journal.pone.0168774.

Dawitz, H. et al. (2020) 'Rcf1 Modulates Cytochrome c Oxidase Activity Especially Under Energy-Demanding Conditions', Frontiers in Physiology, 10, p. 1555. doi: 10.3389/fphys.2019.01555.

Decoster, E. et al. (1990) 'The MSS51 gene product is required for the translation of the 
COX1 mRNA in yeast mitochondria', Molecular and General Genetics MGG, 224(1), pp. 111-118. doi: 10.1007/BF00259457.

Dekker, P. J. et al. (1996) 'Characterization of the preprotein translocase of the outer mitochondrial membrane by blue native electrophoresis', BIOLOGICAL CHEMISTRY HOPPE SEYLER, 377, pp. 535-538.

Dodia, R. et al. (2014) 'Comparisons of subunit 5A and 5B isoenzymes of yeast cytochrome c oxidase', Biochemical Journal, 464(Pt 3), pp. 335-342. doi: 10.1042/BJ20140732.

Dudkina, N. V. et al. (2011) 'Interaction of complexes I, III, and IV within the bovine respirasome by single particle cryoelectron tomography', Proceedings of the National Academy of Sciences, 108(37), pp. 15196-15200. doi: 10.1073/pnas.1107819108.

de Duve, C. et al. (1955) 'Tissue fractionation studies. 6. Intracellular distribution patterns of enzymes in rat-liver tissue', Biochemical Journal, 60(4), pp. 604-617.

Elliott, L. E., Saracco, S. A. and Fox, T. D. (2012) 'Multiple Roles of the Cox20 Chaperone in Assembly of Saccharomyces cerevisiae Cytochrome c Oxidase', Genetics, 190(2), pp. 559-567. doi: 10.1534/genetics.111.135665.

Endres, M., Neupert, W. and Brunner, M. (1999) 'Transport of the ADP/ATP carrier of mitochondria from the TOM complex to the TIM22.54 complex.', The EMBO Journal, 18(12), pp. 3214-3221. doi: 10.1093/emboj/18.12.3214.

Eubel, H., Jänsch, L. and Braun, H.-P. (2003) 'New Insights into the Respiratory Chain of Plant Mitochondria. Supercomplexes and a Unique Composition of Complex II', Plant Physiology, 133(1), pp. 274-286. doi: 10.1104/pp.103.024620.

Falabella, M. et al. (2021) 'Cardiolipin, Mitochondria, and Neurological Disease', Trends in Endocrinology \& Metabolism, 32(4), pp. 224-237. doi: 10.1016/j.tem.2021.01.006.

Fischer, M. and Riemer, J. (2013) 'The Mitochondrial Disulfide Relay System: Roles in Oxidative Protein Folding and Beyond', International Journal of Cell Biology, 2013. doi: $10.1155 / 2013 / 742923$.

Fontanesi, F. et al. (2006) 'Assembly of mitochondrial cytochrome $c$-oxidase, a complicated and highly regulated cellular process', American Journal of PhysiologyCell Physiology, 291(6), pp. C1129-C1147. doi: 10.1152/ajpcell.00233.2006.

Franco, L. V. R. et al. (2018) 'Cox2p of yeast cytochrome oxidase assembles as a standalone subunit with the Cox1p and Cox3p modules', Journal of Biological Chemistry, 293(43), pp. 16899-16911. doi: 10.1074/jbc.RA118.004138.

Fukuda, R. et al. (2007) 'HIF-1 Regulates Cytochrome Oxidase Subunits to Optimize 
Efficiency of Respiration in Hypoxic Cells', Cell, 129(1), pp. 111-122. doi: 10.1016/j.cell.2007.01.047.

Gabaldón, T. and Huynen, M. A. (2004) 'Shaping the mitochondrial proteome', Biochimica et Biophysica Acta (BBA) - Bioenergetics, 1659(2), pp. 212-220. doi: 10.1016/j.bbabio.2004.07.011.

Gabaldón, T. and Pittis, A. A. (2015) 'Origin and evolution of metabolic sub-cellular compartmentalization in eukaryotes', Biochimie, 119, pp. 262-268. doi: 10.1016/j.biochi.2015.03.021.

Gakh, O., Cavadini, P. and Isaya, G. (2002) 'Mitochondrial processing peptidases', Biochimica et Biophysica Acta (BBA) - Molecular Cell Research, 1592(1), pp. 63-77. doi: 10.1016/S0167-4889(02)00265-3.

Gallagher, S. et al. (2004) 'Immunoblotting and Immunodetection', Current Protocols in Neuroscience, 29(1), p. 5.19.1-5.19.24. doi: https://doi.org/10.1002/0471142301.ns0519s29.

García-Poyatos, C. et al. (2020) 'Scaf1 promotes respiratory supercomplexes and metabolic efficiency in zebrafish', EMBO Reports, 21(7). doi: 10.15252/embr.202050287.

Garlich, J. et al. (2017) 'Mutational Analysis of the QRRQ Motif in the Yeast Hig1 Type 2 Protein Rcf1 Reveals a Regulatory Role for the Cytochrome c Oxidase Complex', The Journal of Biological Chemistry, 292(13), pp. 5216-5226. doi: 10.1074/jbc.M116.758045.

Ghosh, A. et al. (2016) 'Mitochondrial disease genes COA6, COX6B and SCO2 have overlapping roles in COX2 biogenesis', Human Molecular Genetics, 25(4), pp. 660671. doi: $10.1093 / \mathrm{hmg} / \mathrm{ddv} 503$.

Gilderson, G. et al. (2003) 'Subunit III of Cytochrome c Oxidase of Rhodobacter sphaeroides Is Required To Maintain Rapid Proton Uptake through the D Pathway at Physiologic pH', Biochemistry, 42(24), pp. 7400-7409. doi: 10.1021/bi0341298.

Gnaiger, E. (2020) 'Mitochondrial Pathways and Respiratory Control: An Introduction to OXPHOS Analysis. 5th ed.' doi: 10.26124/BEC:2020-0002.

Gomkale, R. et al. (2020) 'Defining the Substrate Spectrum of the TIM22 Complex Identifies Pyruvate Carrier Subunits as Unconventional Cargos', Current biology: $C B$, 30(6), pp. 1119-1127.e5. doi: 10.1016/j.cub.2020.01.024.

Grandier-Vazeille, X. et al. (2001) 'Yeast Mitochondrial Dehydrogenases Are Associated in a Supramolecular Complex', Biochemistry, 40(33), pp. 9758-9769. doi: 
10.1021/bi010277r.

Green, M. R. et al. (2012) 'Molecular cloning: a laboratory manual', in Molecular cloning: a laboratory manual, pp. 1890-1890.

Greggio, C. et al. (2017) 'Enhanced Respiratory Chain Supercomplex Formation in Response to Exercise in Human Skeletal Muscle', Cell Metabolism, 25(2), pp. 301-311. doi: 10.1016/j.cmet.2016.11.004.

Guerrero-Castillo, S. et al. (2017) 'The Assembly Pathway of Mitochondrial Respiratory Chain Complex I', Cell Metabolism, 25(1), pp. 128-139. doi: 10.1016/j.cmet.2016.09.002.

Guo, R. et al. (2017) 'Architecture of Human Mitochondrial Respiratory Megacomplex I2III2IV2', Cell, 170(6), pp. 1247-1257.e12. doi: 10.1016/j.cell.2017.07.050.

Hackenbrock, C. R., Chazotte, B. and Gupte, S. S. (1986) 'The random collision model and a critical assessment of diffusion and collision in mitochondrial electron transport', Journal of Bioenergetics and Biomembranes, 18(5), pp. 331-368. doi: 10.1007/BF00743010.

Hartley, A. M. et al. (2019) 'Structure of yeast cytochrome c oxidase in a supercomplex with cytochrome bc 1', Nature Structural \& Molecular Biology, 26(1), pp. 78-83. doi: 10.1038/s41594-018-0172-z.

Hartley, A. M. et al. (2020) 'Rcf2 revealed in cryo-EM structures of hypoxic isoforms of mature mitochondrial III-IV supercomplexes', Proceedings of the National Academy of Sciences of the United States of America, 117(17), pp. 9329-9337. doi: 10.1073/pnas.1920612117.

Hawlitschek, G. et al. (1988) 'Mitochondrial protein import: Identification of processing peptidase and of PEP, a processing enhancing protein', Cell, 53(5), pp. 795-806. doi: 10.1016/0092-8674(88)90096-7.

Hayashi, T. et al. (2015) 'Higdla is a positive regulator of cytochrome c oxidase', Proceedings of the National Academy of Sciences, 112(5), pp. 1553-1558. doi: 10.1073/pnas.1419767112.

Heinemeyer, J. et al. (2007) 'A Structural Model of the Cytochrome c Reductase/Oxidase Supercomplex from Yeast Mitochondria*', Journal of Biological Chemistry, 282(16), pp. 12240-12248. doi: 10.1074/jbc.M610545200.

Herrmann, J. M. and Funes, S. (2005) 'Biogenesis of cytochrome oxidase-Sophisticated assembly lines in the mitochondrial inner membrane', Gene, 354, pp. 43-52. doi: 10.1016/j.gene.2005.03.017. 
Hill, K. et al. (1998) 'Tom40 forms the hydrophilic channel of the mitochondrial import pore for preproteins', Nature, 395(6701), pp. 516-521. doi: 10.1038/26780.

Hoang, N. H. et al. (2019) 'Hypoxia-inducible gene domain 1 proteins in yeast mitochondria protect against proton leak through complex IV', Journal of Biological Chemistry, 294(46), pp. 17669-17677. doi: 10.1074/jbc.RA119.010317.

Hock, D. H. et al. (2020) 'HIGD2A is required for assembly of the COX3 module of human mitochondrial complex IV', Molecular \& Cellular Proteomics, $\mathrm{p}$. mcp.RA120.002076. doi: 10.1074/mcp.RA120.002076.

Hodge, M. R. et al. (1989) 'Inverse regulation of the yeast COX5 genes by oxygen and heme.', Molecular and Cellular Biology, 9(5), pp. 1958-1964.

Horan, S. et al. (2005) 'Analysis of COX2 mutants reveals cytochrome oxidase subassemblies in yeast', Biochemical Journal, 390(Pt 3), pp. 703-708. doi: 10.1042/BJ20050598.

Horten, P., Colina-Tenorio, L. and Rampelt, H. (2020) 'Biogenesis of Mitochondrial Metabolite Carriers', Biomolecules, 10(7). doi: 10.3390/biom10071008.

Huertas, J. R. et al. (2017) 'Antioxidant effect of exercise: Exploring the role of the mitochondrial complex I superassembly', Redox Biology, 13, pp. 477-481. doi: 10.1016/j.redox.2017.07.009.

Ikeda, K. et al. (2013) 'A stabilizing factor for mitochondrial respiratory supercomplex assembly regulates energy metabolism in muscle', Nature Communications, 4(1), p. 2147. doi: $10.1038 /$ ncomms3147.

Janke, C. et al. (2004) 'A versatile toolbox for PCR-based tagging of yeast genes: new fluorescent proteins, more markers and promoter substitution cassettes', Yeast, 21(11), pp. 947-962. doi: https://doi.org/10.1002/yea.1142.

Knop, M. et al. (1999) 'Epitope tagging of yeast genes using a PCR-based strategy: more tags and improved practical routines', Yeast, 15(10B), pp. 963-972. doi: 10.1002/(SICI)1097-0061(199907)15:10B<963::AID-YEA399>3.0.CO;2-W.

Krumschnabel, G. et al. (2015) 'Simultaneous high-resolution measurement of mitochondrial respiration and hydrogen peroxide production', Methods in Molecular Biology (Clifton, N.J.), 1264, pp. 245-261. doi: 10.1007/978-1-4939-2257-4_22.

Kwast, K. E., Burke, P. V. and Poyton, R. O. (1998) 'Oxygen sensing and the transcriptional regulation of oxygen-responsive genes in yeast.', Journal of Experimental Biology, 201(8), pp. 1177-1195.

van der Laan, M. et al. (2007) 'Motor-free mitochondrial presequence translocase drives 
membrane integration of preproteins', Nature Cell Biology, 9(10), pp. 1152-1159. doi: $10.1038 /$ ncb1635.

Laemmli, U. K. (1970) 'SDS-page Laemmli method', Nature, 227, pp. 680-5.

LaMarche, A. E. et al. (1992) 'Isolation and characterization of COX12, the nuclear gene for a previously unrecognized subunit of Saccharomyces cerevisiae cytochrome c oxidase.', Journal of Biological Chemistry, 267(31), pp. 22473-22480. doi: 10.1016/S0021-9258(18)41696-1.

Lane, N. and Martin, W. (2010) 'The energetics of genome complexity', Nature, 467(7318), pp. 929-934. doi: 10.1038/nature09486.

Lapuente-Brun, E. et al. (2013) 'Supercomplex Assembly Determines Electron Flux in the Mitochondrial Electron Transport Chain', Science, 340(6140), pp. 1567-1570. doi: $10.1126 /$ science. 1230381 .

Lazarou, M. et al. (2009) 'Assembly of nuclear DNA-encoded subunits into mitochondrial complex IV, and their preferential integration into supercomplex forms in patient mitochondria', The FEBS Journal, 276(22), pp. 6701-6713. doi: https://doi.org/10.1111/j.1742-4658.2009.07384.x.

Lenaz, G. and Genova, M. L. (2007) 'Kinetics of integrated electron transfer in the mitochondrial respiratory chain: random collisions vs. solid state electron channeling', American Journal of Physiology-Cell Physiology, 292(4), pp. C1221-C1239. doi: 10.1152/ajpcell.00263.2006.

Letts, J. A., Fiedorczuk, K. and Sazanov, L. A. (2016) 'The architecture of respiratory supercomplexes', Nature, 537(7622), pp. 644-648. doi: 10.1038/nature19774.

Levchenko, M. et al. (2016) 'Cox26 is a novel stoichiometric subunit of the yeast cytochrome c oxidase', Biochimica et Biophysica Acta (BBA) - Molecular Cell Research, 1863(7), pp. 1624-1632. doi: 10.1016/j.bbamcr.2016.04.007.

Li, Y. et al. (2007) 'An Assembled Complex IV Maintains the Stability and Activity of Complex I in Mammalian Mitochondria', Journal of Biological Chemistry, 282(24), pp. 17557-17562. doi: 10.1074/jbc.M701056200.

Linden, A. et al. (2020) 'A cross-linking mass spectrometry approach defines protein interactions in yeast mitochondria', Molecular \& Cellular Proteomics, p. mcp.RA120.002028. doi: 10.1074/mcp.RA120.002028.

Lobo-Jarne, T. et al. (2018) 'Human COX7A2L Regulates Complex III Biogenesis and Promotes Supercomplex Organization Remodeling without Affecting Mitochondrial Bioenergetics', Cell reports, 25(7), pp. 1786-1799.e4. doi: 
10.1016/j.celrep.2018.10.058.

Lobo-Jarne, T. and Ugalde, C. (2018) 'Respiratory chain supercomplexes: Structures, function and biogenesis', Seminars in Cell \& Developmental Biology, 76, pp. 179-190. doi: 10.1016/j.semcdb.2017.07.021.

Lode, A. et al. (2000) 'Mitochondrial copper metabolism in yeast: interaction between Sco1p and Cox2p', FEBS Letters, 485(1), pp. 19-24. doi: https://doi.org/10.1016/S0014-5793(00)02176-1.

Longtine, M. S. et al. (1998) 'Additional modules for versatile and economical PCR-based gene deletion and modification in Saccharomyces cerevisiae', Yeast, 14(10), pp. 953961. doi: 10.1002/(SICI)1097-0061(199807)14:10<953::AID-YEA293>3.0.CO;2-U.

Lytovchenko, O. et al. (2013) 'Signal recognition initiates reorganization of the presequence translocase during protein import', The EMBO Journal, 32(6), pp. 886898. doi: 10.1038/emboj.2013.23.

Malhotra, K. et al. (2013) 'Structural changes in the mitochondrial Tim23 channel are coupled to the proton-motive force', Nature Structural \& Molecular Biology, 20(8), pp. 965-972. doi: 10.1038/nsmb.2613.

Malina, C., Larsson, C. and Nielsen, J. (2018) 'Yeast mitochondria: an overview of mitochondrial biology and the potential of mitochondrial systems biology', FEMS Yeast Research, 18(foy040). doi: 10.1093/femsyr/foy040.

Manthey, G. M. and McEwen, J. E. (1995) 'The product of the nuclear gene PET309 is required for translation of mature mRNA and stability or production of introncontaining RNAs derived from the mitochondrial COX1 locus of Saccharomyces cerevisiae', The EMBO journal, 14(16), pp. 4031-4043.

Maranzana, E. et al. (2013) 'Mitochondrial Respiratory Supercomplex Association Limits Production of Reactive Oxygen Species from Complex I', Antioxidants \& Redox Signaling, 19(13), pp. 1469-1480. doi: 10.1089/ars.2012.4845.

Martin, J., Mahlke, K. and Pfanner, N. (1991) 'Role of an energized inner membrane in mitochondrial protein import. Delta psi drives the movement of presequences.', Journal of Biological Chemistry, 266(27), pp. 18051-18057. doi: 10.1016/S00219258(18)55235-2.

Matus-Ortega, M. G. et al. (2015) 'New complexes containing the internal alternative NADH dehydrogenase (Ndi1) in mitochondria of Saccharomyces cerevisiae', Yeast, 32(10), pp. 629-641. doi: https://doi.org/10.1002/yea.3086.

McStay, G. P. et al. (2013) 'Characterization of Assembly Intermediates Containing 
Subunit 1 of Yeast Cytochrome Oxidase*', Journal of Biological Chemistry, 288(37), pp. 26546-26556. doi: 10.1074/jbc.M113.498592.

McStay, G. P., Su, C. H. and Tzagoloff, A. (2013) 'Modular assembly of yeast cytochrome oxidase', Molecular Biology of the Cell. Edited by T. D. Fox, 24(4), pp. 440-452. doi: 10.1091/mbc.e12-10-0749.

Meisinger, C., Pfanner, N. and Truscott, K. N. (2006) 'Isolation of yeast mitochondria', Methods in Molecular Biology (Clifton, N.J.), 313, pp. 33-39. doi: 10.1385/1-59259958-3:033.

Merkley, E. D. et al. (2014) 'Distance restraints from crosslinking mass spectrometry: Mining a molecular dynamics simulation database to evaluate lysine-lysine distances', Protein Science : A Publication of the Protein Society, 23(6), pp. 747-759. doi: $10.1002 /$ pro. 2458 .

Mesecke, N. et al. (2005) 'A Disulfide Relay System in the Intermembrane Space of Mitochondria that Mediates Protein Import', Cell, 121(7), pp. 1059-1069. doi: 10.1016/j.cell.2005.04.011.

Mick, D. U. et al. (2007) 'Shy1 couples Cox1 translational regulation to cytochrome c oxidase assembly', The EMBO Journal, 26(20), pp. 4347-4358. doi: 10.1038/sj.emboj.7601862.

Mick, D. U. et al. (2010) 'Coa3 and Cox14 are essential for negative feedback regulation of COX1 translation in mitochondria', Journal of Cell Biology, 191(1), pp. 141-154. doi: $10.1083 /$ jcb.201007026.

Mick, D. U., Fox, T. D. and Rehling, P. (2011) 'Inventory control: cytochrome oxidase assembly regulates mitochondrial translation', Nature Reviews. Molecular Cell Biology, 12(1), pp. 14-20. doi: 10.1038/nrm3029.

Milenkovic, D. et al. (2017) 'The Enigma of the Respiratory Chain Supercomplex', Cell Metabolism, 25(4), pp. 765-776. doi: 10.1016/j.cmet.2017.03.009.

Mileykovskaya, E. et al. (2012) 'Arrangement of the respiratory chain complexes in Saccharomyces cerevisiae supercomplex III2IV2 revealed by single particle cryoelectron microscopy', The Journal of Biological Chemistry, 287(27), pp. 23095-23103. doi: 10.1074/jbc.M112.367888.

Miller, W. L. (2013) 'Steroid hormone synthesis in mitochondria', Molecular and Cellular Endocrinology, 379(1), pp. 62-73. doi: 10.1016/j.mce.2013.04.014.

Mitchell, P. (1976) 'Possible molecular mechanisms of the protonmotive function of cytochrome systems', Journal of Theoretical Biology, 62(2), pp. 327-367. doi: 
$10.1016 / 0022-5193(76) 90124-7$.

Moreno-Lastres, D. et al. (2012) 'Mitochondrial Complex I Plays an Essential Role in Human Respirasome Assembly', Cell Metabolism, 15(3), pp. 324-335. doi: 10.1016/j.cmet.2012.01.015.

Morgenstern, M. et al. (2017) 'Definition of a High-Confidence Mitochondrial Proteome at Quantitative Scale', Cell Reports, 19(13), pp. 2836-2852. doi:

10.1016/j.celrep.2017.06.014.

Mourier, A. et al. (2014) 'The Respiratory Chain Supercomplex Organization Is Independent of COX7a21 Isoforms', Cell Metabolism, 20(6), pp. 1069-1075. doi: 10.1016/j.cmet.2014.11.005.

Müller, P. P. et al. (1984) 'A nuclear mutation that post-transcriptionally blocks accumulation of a yeast mitochondrial gene product can be suppressed by a mitochondrial gene rearrangement', Journal of Molecular Biology, 175(4), pp. 431452. doi: 10.1016/0022-2836(84)90178-5.

Ndi, M. et al. (2018) 'Biogenesis of the bc1 Complex of the Mitochondrial Respiratory Chain', Journal of Molecular Biology, 430(21), pp. 3892-3905. doi: 10.1016/j.jmb.2018.04.036.

Nelson, D. and Cox, M. (2009) Lehninger Biochemie. 4th edn. Berlin Heidelberg: Springer-Verlag (Springer-Lehrbuch). Available at: https://www.springer.com/de/book/9783540686378 (Accessed: 29 March 2021).

Netter, P. et al. (1982) 'Critical sequences within mitochondrial introns: Cis-dominant mutations of the "cytochrome-b-like" intron of the oxidase gene', Cell, 28(4), pp. 733738. doi: 10.1016/0092-8674(82)90052-6.

Neupane, P. et al. (2019) 'ATP Synthase: Structure, Function and Inhibition', Biomolecular Concepts, 10(1), pp. 1-10. doi: 10.1515/bmc-2019-0001.

Nolfi-Donegan, D., Braganza, A. and Shiva, S. (2020) 'Mitochondrial electron transport chain: Oxidative phosphorylation, oxidant production, and methods of measurement', Redox Biology, 37. doi: 10.1016/j.redox.2020.101674.

Ott, M. and Herrmann, J. M. (2010) 'Co-translational membrane insertion of mitochondrially encoded proteins', Biochimica et Biophysica Acta (BBA) - Molecular Cell Research, 1803(6), pp. 767-775. doi: 10.1016/j.bbamcr.2009.11.010.

Pérez-Pérez, R. et al. (2016) 'COX7A2L Is a Mitochondrial Complex III Binding Protein that Stabilizes the III2+IV Supercomplex without Affecting Respirasome Formation', Cell Reports, 16(9), pp. 2387-2398. doi: 10.1016/j.celrep.2016.07.081. 
Pfeiffer, K. et al. (2003) 'Cardiolipin Stabilizes Respiratory Chain Supercomplexes*', Journal of Biological Chemistry, 278(52), pp. 52873-52880. doi: 10.1074/jbc.M308366200.

Pierrel, F. et al. (2007) 'Coal links the Mss51 post-translational function to Cox1 cofactor insertion in cytochrome c oxidase assembly', The EMBO Journal, 26(20), pp. 43354346. doi: 10.1038/sj.emboj.7601861.

Protasoni, M. et al. (2020) 'Respiratory supercomplexes act as a platform for complex IIImediated maturation of human mitochondrial complexes I and IV', The EMBO Journal, 39(3), p. e102817. doi: 10.15252/embj.2019102817.

Rappocciolo, E. and Stiban, J. (2019) 'Prokaryotic and Mitochondrial Lipids: A Survey of Evolutionary Origins', in Stiban, J. (ed.) Bioactive Ceramides in Health and Disease: Intertwined Roles of Enigmatic Lipids. Cham: Springer International Publishing (Advances in Experimental Medicine and Biology), pp. 5-31. doi: 10.1007/978-3-03021162-2_2.

Rathore, S. et al. (2019) 'Cryo-EM structure of the yeast respiratory supercomplex', Nature Structural \& Molecular Biology, 26(1), pp. 50-57. doi: 10.1038/s41594-0180169-7.

Rehling, P., Brandner, K. and Pfanner, N. (2004) 'Mitochondrial import and the twin-pore translocase', Nature Reviews Molecular Cell Biology, 5(7), pp. 519-530. doi: $10.1038 / \mathrm{nrm} 1426$.

Reifschneider, N. H. et al. (2006) 'Defining the Mitochondrial Proteomes from Five Rat Organs in a Physiologically Significant Context Using 2D Blue-Native/SDS-PAGE', Journal of Proteome Research, 5(5), pp. 1117-1132. doi: 10.1021/pr0504440.

Rich, P. R. and Maréchal, A. (2010) 'The mitochondrial respiratory chain', Essays in Biochemistry. Edited by G. C. Brown and M. P. Murphy, 47, pp. 1-23. doi: 10.1042/bse0470001.

Richter-Dennerlein, R. et al. (2016) 'Mitochondrial Protein Synthesis Adapts to Influx of Nuclear-Encoded Protein', Cell, 167(2), pp. 471-483.e10. doi: 10.1016/j.cell.2016.09.003.

Romero-Garcia, S. and Prado-Garcia, H. (2019) 'Mitochondrial calcium: Transport and modulation of cellular processes in homeostasis and cancer (Review)', International Journal of Oncology, 54(4), pp. 1155-1167. doi: 10.3892/ijo.2019.4696.

Römpler, K. et al. (2016) 'Overlapping Role of Respiratory Supercomplex Factor Rcf2 and Its N-terminal Homolog Rcf3 in Saccharomyces cerevisiae', Journal of Biological 
Chemistry, 291(45), pp. 23769-23778. doi: 10.1074/jbc.M116.734665.

Römpler, K. M. (2016) 'Dynamic changes in cytochrome c oxidase assembly and organization'.

Ryan, M. T., Voos, W. and Pfanner, N. (2001) 'Assaying protein import into mitochondria', Methods Cell Biol, 65(2001), pp. 189-215.

Rydström Lundin, C. et al. (2016) 'Regulatory role of the respiratory supercomplex factors in Saccharomyces cerevisiae', Proceedings of the National Academy of Sciences, 113(31), pp. E4476-E4485. doi: 10.1073/pnas.1601196113.

Rydström Lundin, C. and Brzezinski, P. (2017) 'Modulation of O2 reduction in Saccharomyces cerevisiae mitochondria', FEBS Letters, 591(24), pp. 4049-4055. doi: 10.1002/1873-3468.12918.

Schäfer, J. et al. (2018) 'Regulation of cytochrome c oxidase activity by modulation of the catalytic site', Scientific Reports, 8(1), p. 11397. doi: 10.1038/s41598-018-29567-4.

Schägger, H. (2006) 'Tricine-sds-page', Nature protocols, 1(1), p. 16.

Schägger, H. and von Jagow, G. (1991) 'Blue native electrophoresis for isolation of membrane protein complexes in enzymatically active form', Analytical biochemistry, 199(2), pp. 223-231.

Schägger, H. and Pfeiffer, K. (2000) 'Supercomplexes in the respiratory chains of yeast and mammalian mitochondria', The EMBO Journal, 19(8), pp. 1777-1783. doi: 10.1093/emboj/19.8.1777.

Schägger, H. and Pfeiffer, K. (2001) 'The Ratio of Oxidative Phosphorylation Complexes I-V in Bovine Heart Mitochondria and the Composition of Respiratory Chain Supercomplexes*', Journal of Biological Chemistry, 276(41), pp. 37861-37867. doi: 10.1074/jbc.M106474200.

Schapira, A. H. (2006) 'Mitochondrial disease', The Lancet, 368(9529), pp. 70-82. doi: 10.1016/S0140-6736(06)68970-8.

Schenkel, L. C. and Bakovic, M. (2014) 'Formation and Regulation of Mitochondrial Membranes', International Journal of Cell Biology, 2014, p. e709828. doi: $10.1155 / 2014 / 709828$.

Schindelin, J. et al. (2012) 'Fiji: an open-source platform for biological-image analysis', Nature methods, 9(7), pp. 676-682.

Schulz, C., Schendzielorz, A. and Rehling, P. (2015) 'Unlocking the presequence import pathway', Trends in Cell Biology, 25(5), pp. 265-275. doi: 10.1016/j.tcb.2014.12.001. Semenza, G. L. (2007) 'Oxygen-dependent regulation of mitochondrial respiration by 
hypoxia-inducible factor 1', Biochemical Journal, 405(1), pp. 1-9. doi: 10.1042/BJ20070389.

Shinzawa-Itoh, K. et al. (2007) 'Structures and physiological roles of 13 integral lipids of bovine heart cytochrome c oxidase', The EMBO Journal, 26(6), pp. 1713-1725. doi: 10.1038/sj.emboj.7601618.

Shiota, T. et al. (2015) 'Molecular architecture of the active mitochondrial protein gate', Science, 349(6255), pp. 1544-1548. doi: 10.1126/science.aac6428.

Sikorski, R. S. and Hieter, P. (1989) 'A system of shuttle vectors and yeast host strains designed for efficient manipulation of DNA in Saccharomyces cerevisiae', Genetics, 122(1), pp. 19-27.

Singhal, R. K. et al. (2017) 'Coil is a novel assembly factor of the yeast complex IIIcomplex IV supercomplex', Molecular Biology of the Cell, 28(20), pp. 2609-2622. doi: 10.1091/mbc.e17-02-0093.

Stehling, O. and Lill, R. (2013) 'The Role of Mitochondria in Cellular Iron-Sulfur Protein Biogenesis: Mechanisms, Connected Processes, and Diseases', Cold Spring Harbor Perspectives in Biology, 5(8). doi: 10.1101/cshperspect.a011312.

Stephan, K. and Ott, M. (2020) 'Timing of dimerization of the bc1 complex during mitochondrial respiratory chain assembly', Biochimica Et Biophysica Acta. Bioenergetics, 1861(5-6), p. 148177. doi: 10.1016/j.bbabio.2020.148177.

Stiller, S. B. et al. (2016) 'Mitochondrial OXA Translocase Plays a Major Role in Biogenesis of Inner-Membrane Proteins', Cell Metabolism, 23(5), pp. 901-908. doi: 10.1016/j.cmet.2016.04.005.

Strecker, V. et al. (2016) 'Supercomplex-associated Cox26 protein binds to cytochrome c oxidase', Biochimica et Biophysica Acta (BBA) - Molecular Cell Research, 1863(7), pp. 1643-1652. doi: 10.1016/j.bbamcr.2016.04.012.

Strogolova, V. et al. (2012) 'Rcf1 and Rcf2, Members of the Hypoxia-Induced Gene 1 Protein Family, Are Critical Components of the Mitochondrial Cytochrome bc1Cytochrome c Oxidase Supercomplex', Molecular and Cellular Biology, 32(8), pp. 1363-1373. doi: 10.1128/MCB.06369-11.

Strogolova, V. et al. (2019) 'The yeast mitochondrial proteins Rcf1 and Rcf2 support the enzymology of the cytochrome $c$ oxidase complex and generation of the proton motive force', Journal of Biological Chemistry, p. jbc.RA118.006888. doi: 10.1074/jbc.RA118.006888.

Stroh, A. et al. (2004) 'Assembly of Respiratory Complexes I, III, and IV into NADH 
Oxidase Supercomplex Stabilizes Complex I in Paracoccus denitrificans*', Journal of Biological Chemistry, 279(6), pp. 5000-5007. doi: 10.1074/jbc.M309505200.

Stuart, R. A. et al. (2000) 'The Cytochrome bc 1 and Cytochromec Oxidase Complexes Associate to Form a Single Supracomplex in Yeast Mitochondria*', Journal of Biological Chemistry, 275(24), pp. 18093-18098. doi: 10.1074/jbc.M001901200.

Su, C.-H., McStay, G. P. and Tzagoloff, A. (2014) 'The Cox3p assembly module of yeast cytochrome oxidase', Molecular Biology of the Cell. Edited by T. D. Fox, 25(7), pp. 965-976. doi: 10.1091/mbc.e13-10-0575.

Suárez-Rivero, J. M. et al. (2016) 'Mitochondrial Dynamics in Mitochondrial Diseases', Diseases, 5(1). doi: 10.3390/diseases5010001.

Svensson-Ek, M. et al. (2002) 'The X-ray Crystal Structures of Wild-type and EQ(I-286) Mutant Cytochrome c Oxidases from Rhodobacter sphaeroides', Journal of Molecular Biology, 321(2), pp. 329-339. doi: 10.1016/S0022-2836(02)00619-8.

Timón-Gómez, A., Garlich, J., et al. (2020a) 'Distinct Roles of Mitochondrial HIGD1A and HIGD2A in Respiratory Complex and Supercomplex Biogenesis', Cell Reports, 31(5), p. 107607. doi: 10.1016/j.celrep.2020.107607.

Timón-Gómez, A., Bartley-Dier, E. L., et al. (2020b) 'HIGD-Driven Regulation of Cytochrome c Oxidase Biogenesis and Function', Cells, 9(12), p. 2620. doi: $10.3390 /$ cells9122620.

Tiranti, V. et al. (2000) 'A novel frameshift mutation of the mtDNA COIII gene leads to impaired assembly of cytochrome c oxidase in a patient affected by Leigh-like syndrome', Human Molecular Genetics, 9(18), pp. 2733-2742. doi: 10.1093/hmg/9.18.2733.

Trendeleva, T. A., Aliverdieva, D. A. and Zvyagilskaya, R. A. (2014) 'Mechanisms of sensing and adaptive responses to low oxygen conditions in mammals and yeasts', Biochemistry (Moscow), 79(8), pp. 750-760. doi: 10.1134/S0006297914080033.

Tsukihara, T. et al. (1996) 'The Whole Structure of the 13-Subunit Oxidized Cytochrome c Oxidase at 2.8 A', Science, 272(5265), pp. 1136-1144. doi: 10.1126/science.272.5265.1136.

Turrens, J. F. (2003) 'Mitochondrial formation of reactive oxygen species', The Journal of Physiology, 552(Pt 2), pp. 335-344. doi: 10.1113/jphysiol.2003.049478.

Tzagoloff, A. et al. (2000) 'Identification of Cox20p, a Novel Protein Involved in the Maturation and Assembly of Cytochrome Oxidase Subunit 2*, Journal of Biological Chemistry, 275(7), pp. 4571-4578. doi: 10.1074/jbc.275.7.4571. 
Varanasi, L. et al. (2006) 'Altering Conserved Lipid Binding Sites in Cytochrome $c$ Oxidase of Rhodobacter sphaeroides Perturbs the Interaction between Subunits I and III and Promotes Suicide Inactivation of the Enzyme †', Biochemistry, 45(50), pp. 1489614907. doi: 10.1021/bi061390q.

Vogel, F. et al. (2006) 'Dynamic subcompartmentalization of the mitochondrial inner membrane', Journal of Cell Biology, 175(2), pp. 237-247. doi: 10.1083/jcb.200605138.

Vögtle, F.-N. et al. (2012) 'Intermembrane Space Proteome of Yeast Mitochondria', Molecular \& Cellular Proteomics : MCP, 11(12), pp. 1840-1852. doi: 10.1074/mcp.M112.021105.

Vukotic, M. et al. (2012) 'Rcf1 Mediates Cytochrome Oxidase Assembly and Respirasome Formation, Revealing Heterogeneity of the Enzyme Complex', Cell Metabolism, 15(3), pp. 336-347. doi: 10.1016/j.cmet.2012.01.016.

Walther, D. M. and Rapaport, D. (2009) 'Biogenesis of mitochondrial outer membrane proteins', Biochimica et Biophysica Acta (BBA) - Molecular Cell Research, 1793(1), pp. 42-51. doi: 10.1016/j.bbamcr.2008.04.013.

Wang, C. and Youle, R. J. (2009) 'The Role of Mitochondria in Apoptosis', Annual review of genetics, 43, pp. 95-118. doi: 10.1146/annurev-genet-102108-134850.

Wang, J. et al. (2006) 'Pancreatic $\beta$ cells lack a low glucose and O2-inducible mitochondrial protein that augments cell survival', Proceedings of the National Academy of Sciences, 103(28), pp. 10636-10641.

Weishaupt, A. and Kadenbach, B. (1992) 'Selective removal of subunit VIb increases the activity of cytochrome c oxidase', Biochemistry, 31(46), pp. 11477-11481. doi: 10.1021/bi00161a028.

Westermann, B., Herrmann, J. M. and Neupert, W. (2001) 'Analysis of mitochondrial translation products in Vivo and in organello in yeast', in Methods in Cell Biology. Academic Press (Mitochondria), pp. 429-438. doi: 10.1016/S0091-679X(01)65025-8.

Wiedemann, N. and Pfanner, N. (2017) 'Mitochondrial Machineries for Protein Import and Assembly', Annual Review of Biochemistry, 86(1), pp. 685-714. doi: 10.1146/annurevbiochem-060815-014352.

Wittig, I., Braun, H.-P. and Schägger, H. (2006) 'Blue native PAGE', Nature protocols, 1(1), p. 418.

Yamano, K. et al. (2008) 'Tom20 and Tom22 Share the Common Signal Recognition Pathway in Mitochondrial Protein Import*', Journal of Biological Chemistry, 283(7), pp. 3799-3807. doi: 10.1074/jbc.M708339200. 
Zhao, R.-Z. et al. (2019) 'Mitochondrial electron transport chain, ROS generation and uncoupling (Review)', International Journal of Molecular Medicine, 44(1), pp. 3-15. doi: $10.3892 /$ ijmm.2019.4188.

Zhou, S., Pettersson, P., Brzezinski, P., et al. (2018a) 'NMR Study of Rcf2 Reveals an Unusual Dimeric Topology in Detergent Micelles', ChemBioChem, 19(5), pp. 444-447. doi: 10.1002/cbic.201700664.

Zhou, S., Pettersson, P., Huang, J., et al. (2018b) 'Solution NMR structure of yeast Rcf1, a protein involved in respiratory supercomplex formation', Proceedings of the National Academy of Sciences, 115(12), pp. 3048-3053. doi: 10.1073/pnas.1712061115.

Zhou, S. et al. (2020) 'NMR Structure and Dynamics Studies of Yeast Respiratory Supercomplex Factor 2', Structure. doi: 10.1016/j.str.2020.08.008.

Zinser, E. et al. (1991) 'Phospholipid synthesis and lipid composition of subcellular membranes in the unicellular eukaryote Saccharomyces cerevisiae.', Journal of Bacteriology, 173(6), pp. 2026-2034. doi: 10.1128/jb.173.6.2026-2034.1991.

Zitomer, R. S., Carrico, P. and Deckert, J. (1997) 'Regulation of hypoxic gene expression in yeast', Kidney International, 51(2), pp. 507-513. doi: 10.1038/ki.1997.71.

Zong, S. et al. (2018) 'Structure of the intact 14-subunit human cytochrome c oxidase', Cell Research, 28(10), pp. 1026-1034. doi: 10.1038/s41422-018-0071-1. 



\section{Acknowledgements}

I'm grateful for each and every support, advice and help I got from so many persons throughout the last years. Without them, this work would not have been possible. I especially thank ...

... Markus Deckers for bringing me to Göttingen and opening the interesting field of mitochondria for me. He offered me the project and position and by this, encouraged me to intensify my interest and knowledge of protein biochemistry. He was never short of ideas and supported me with his abundance of patience and his ability to show me the positive outcome of an experiment when I couldn't see it. Although he left the lab to Hamburg, he gave his best to keep up with the supervision.

... Peter Rehling for not hesitating to include me into his group. He adopted me right away and was always a helpful and valuable advisor throughout the whole projects. When Markus left the lab, he immediately stepped up that I won't feel left-behind and gave me the opportunity to finish my $\mathrm{PhD}$ without big problems.

... Dörthe Katschinski and Stefan Jakobs for their support and helpful discussions during the thesis committee meetings. And together with them, Ralph Kehlenbach, Dieter Klopfenstein and Henning Urlaub for taking the time and join my extended thesis committee for the disputation.

... all collaborators who were all highly motivated to help me, independent of the results making it into my thesis. Andreas Linden, for great discussions and help with the mass spectrometry results. Michael Müller and his medical students (especially Lena), for introducing me to new methods of ROS measurements. Hien Ho Dieu, for helping me with Oroboros measurements. Akos Farkas, for his support with the deletion library and microscopic analysis.

... all current and former members of the Institute of Cellular Biochemistry for sharing the experimental and scientific expertise. Although Corona changed it a bit - the daily working routine is much more fun together with them; not only with the Rehling group but also Thumm's, Richter-Dennerlein's, Meinecke's and Schu's. Mirjam, for all her technical and psychological support and providing me with her special gadgets and tricks for everything. Carmen and Annette, for being the superheroes behind the scenes and taking so much work 
away from all of us. Special thank goes to Markus, Sabine, Ay-Lin, Daniel and David for critically reading my work. Sabine (or is it Bettina?!), also for being my lab twin throughout the whole time, always feeling what I was feeling and understanding exactly all the obstacles I was facing during this work. Along the same lines, Tobi and Lio as former lab members, for especially shaping the time at the beginning of this thesis. Ridhima, for helping me with yeast but also a lot of other questions. David and Markus, for not only being scientific advisors but also open for private discussions with a beer in the hand.

... My family and friends, for never being tired of showing support and open ears.

... Daniel, for being the best and trying to understand every bit of my work. 